\section{JUN 221931 Sunongis}

\section{FAUNAE/SCANDINA VIAE.}

76,015

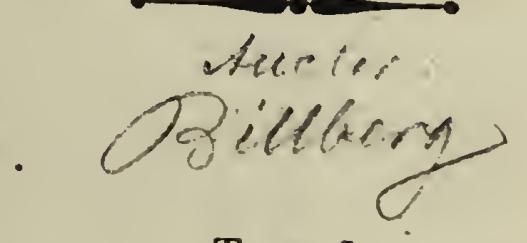

Tом. I.

Pans 2.

\section{A VES.}

- ANIMALIA sanguino calido, rubro; pulmone respirantia : ova ponentia, e quibus pulli ereniunt.

HOLMIE, 1828 .

Ex officine typogr. CAMOLE DExs=T. 


$$
\begin{aligned}
& 59+11: 418 \\
& \text { whenge phen om }
\end{aligned}
$$

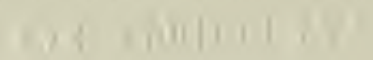




\section{Subclassis r. GEORNITHES, Ordo r. TRECHOPODES,} Natio I. O'E[DIJES, Genus I. OT(S *), Si. Trapp.

species r. turdn: pallide rufa: fusciis nigris; m. capite coilngle cantescentilus: man dibula inferiore ulrindue longe mystacea.

- $f$. vertice fulvo-nigronuc-striato; mystaci. bus mulis.

Synonyma: Linnei Systema Naturx edit. XII Vol. r. pag. 264. n. I. Retzii Fauna Srecic, no $17 \mathrm{~S}$ Nilssons Or-

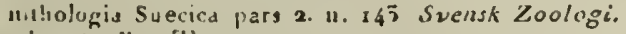
Sveris: trög 'Trapl.

IIalutat in campis arenosis Scanize rarissime (D. Retrius'. Magnitudinem Preudotannis splvestris stperat, vel Inneitudine it pedum; ora $2-3$ brumescentia, maculis rubrescentibus, in agris ponens, pullos post dies 25 excludit. Tantum irats et tentpore coëundi sonat. Grans diversa, glamiua olmraque, insecta rern que cibus ejus suat Carn sspida. - Kushum maris fusceocens. Sub lingua furameu in saccun gularem pro equa

-) Habitus generis: Corpus robuseurn, ovalum : pedibus j-dartylis elongatis; femuribus plerumque parie inferiore nudis. 1lip mediocres: remige 3:0 longissimo. Kererices plures quam 12, seu 18-20. Nomen genericun Aristobelis' It tis, Avis cards. 
servanda dicens, Remiges primores nigri albo-fasciati. Scapulares et tectrices alarum minores pallide.rifie, lligro undulalie. Abdomen, crissum et lestrices slaruni majores altre. Hectrices 20 eminentins :ulo: Casciis maculisque nigris; apice lutescentes. Peles grisrscantes Iris autantia.

2. tetrax: mandibulis inb(ub)ibu; supra pallide vufo nigioque-variegata; $m$. collo $u$ gro: torque alloo.

f. collo et dorso concolorilus:

Linn. S. N 264. 3. - Faina Suecica 196 , Relz. Fu. 179. Nilss. Orn. 144. S2. Zool.

Sv. Dverg'Traple.

Junior $m$. collo dorsoque concoloribus alsque torque albo.

Hob. pluribus in locis lempore vernali, quamvis rara. Magnitudine corporis fele nimol quam Ternomis Tetricis I longiladine is po!l. In telta ova 3 -5 viridis nitida ponit. Nocte sonal $p$ rue, $p: u t$. Corpus supra nigrum: siriis pallide rufis; temposibus. gula senisplle rulescente-albis: maculis parvis nigris. Abjoman $t$ crissum in. alta, $f$. alba nigrolasciata el maculata.

\section{Ord. 2. ELAZOPODES,} Trib. I. APSIEFAMPHAE,

Nat. 2. 'TETRAONIDES,

Divisio x. libiis nudis:

C. 2. PERDIX *), Sv. Rapphönz.

-) pedibus calcaralis:

3. cinerea: cinereo, rufo uigroque varia:

-) Ilab. giener omnino Tetraonis, sed pedilus nudis, maris plerunque calcaratis; alis brevibus remigibus 5 , interdum 5 longissimis; cunda rectici bus 18 l. 14 , Lrevi, rotundata. - N. gen. Intilitim marula sub nculis murla coccinca; pecince caslaneo; randa reciriciis mediis execptis rufe. scente; m. macula pectorali Jumata.

$f$ : macula pectorali magria rotundata, te. clricibus alarum scapulis finsco-maculatis

Linn. S. A. 276. 13. Fn. 205. Tietr. Fn. 88 . Hnmmer Farina Norvegica. 3. 46, n. 218 . Tetrao Perdix Nilss. Ort. I. St. sn Zool.

Sv. askgrit Ra pphän a, Âkerhöna. Norregis: Rajplïne. Agrvönuc.

Jun. sime macula peciorali; perlibus flare. scentilurs.

Hab in agris gregatin; sumra sconiam, nici il.rm. ducta, rarissima. Insectis vermibusqun, semiribus, her. bis, graminitos varis baccisgue junjemints veltit. In stalu coëundil. mense martio bini sirunt. Jin. ova iz-2x sordide virescente-grisea in perra pmit. Mlas clanilat girlläh et fem gärl. A congeneribus lungiodian 12 poll., macula castanea pectorali nox difuoscitur. Ii, rufo-fusca. Reclrices medice 4 lutescente liscoque vaiegara lasciis castaneis.

$$
\text { *) pedibus ecelcarais: }
$$

2. coturnix: pallicle müscens: macul is nigris, striis longitudinalibus albirantibus stri* aque supra oculos $c t$ in rerije alba: rnuda fusca striis transversis rachibusque albisuntibus; m. gula nigra.

$f$ gula alba; pectore maculis n:gricantibus. Lina. S. N. 278. 20 Fn. 23G. lietz. Fn. 129 Hamm Fn。 219. 'Teirao. Nilss. Oin. Y42. Sw Zool

Sv. Waktelfa pplïina. N. Vartel.

Ilab. in arvis australioribus non frequens, mierain. ria; inter segetes clamilaus: pickervick, picker vick! et mas in tempore aitus: primo värru, lër. ra! dein clara vuca byc den ryck! timidus $\varepsilon^{i l-}$ io, lerrore micans Trylrockreas! pelliciens vytyvi. 
2 $--$ 

men ovis formicarum vicentar Cladacter bus indicatis cum rosirn nignicante, uaribus longitudinalibus unembiona fornicata inflata, tectis pedibusque pallide incarualis, absyue calcate, Jongitudineque tallum cisca 7 poll. bene cognila. Iris olivaceo-fusca.

Div. 2. libiis plumosis:

G. 3. TETRAO *), Sv. Orre.

-) digitis glabris:

1. urogallus: axillis albis; canda cuucatorotundata; m. nigricasss supua telutissimc albo variegatus; sula barbita.

$f$. stpra rufescente nigroque-transversina urdulata, subtus eminentius rufescens, nigroundulata.

Linn. S. N. 275. I. Fn, 200. Retz. Fu, 183. Nilss. Orn. 156. Homm. Fin 2r5.- Puncopididens Nosnes NalurI g̈e flistoria Del. s. iuq. Sv. livol.

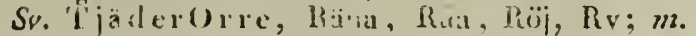

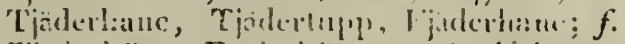

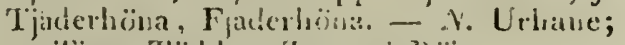

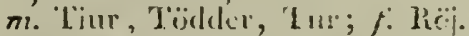

Parielos 13. dorso silperine aibo fermeiutoque-malulato, inferione nigre: penus atibis insertis; abdominc cauciaque allo-1iaeulatis.

Elom in Act. Halm. 18c7. o Nilss. 1. c. $-\alpha$ Sv. Zool.

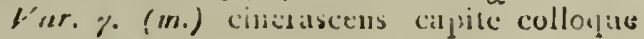
sittuatioribtis.

-) ll, bitis fere Pspululaonis sylrostris. ied rallo brelibure wlis brevilus rumigilus 3 , I longissimis, pe. dibus placinosis ec rectricilus is l. aj. - N. gener. risuscim labinuruin, Gusuel.

Thunierg in Act. IToim. 1789.979 . Nilss. 1. c. $-B$ Siv. \%ool.

Vur. $\delta$, f. supra sordide tes'acciss: $n 1$ :dulis ratoribus aibis; subius testacto albuloque-maculiatus.

Nilss. 1. c. $-\gamma$ sv. '/u '.

Vur. \&. (f.) totus sordide albidus, fuscescente-n(i):alwis.

Nilss. 1. c. $-\delta$ Sv Znol

Hab. in sylvis lese ub jue montosig vastis; rarietas rameri 3 :n Dulecar:ia, coterx in Lanponia rarisime;

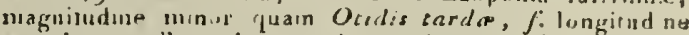
2 poi ro poll, sed in inulto inajor. Baccis, gemmis. Frumentis, granis, folis abieris inseclisque victitgt. Menso April, coeulir: fern. 8.12 ova sord de alba maculis surdide luteis III terr, funit, qux 4 septimanas fovet $m$. in boc statu tem!nte matutuo l. In:ra 2 ramos arbormon cauda erecta pl pecture eflusto colcausvecal feminam sonu Klacechtdode, dode.dodel, diodel-dodetrre. Elack! ind. hedeludehe-hedehedehe-hedehedehej! - f responder gack gark gack gäck

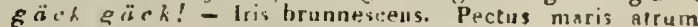

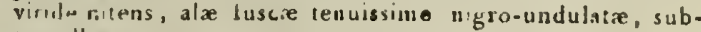
tus a'bs.

2. tetrix: specula alarum alba, cauda bifurca: $m$. ater: capite, collo, dorso unpy rio. que nitorc carulen-splendentibus.

f. capite colloque pallide rubis nigro-lancia. tis: dorso mopygio cartaque nioris pallide. rufo-firciatis.

Linn.S. N. 274. 2. Fn. 202. Retz. Fin. 184. Nilss. Dru.rin. llamni. Fu. 215. Honcopp. N. II. 2. 104. Sv. Koul. 39.

Sv. egentlig Orre, Ort, Werc; m. ()re ltase, Orrtupp; $f$. Orhöna. N. Aarfil;', Orvlig!, Arcfugl; m. Larlianc; $f$. Aut:löue. 

Var. ß. (m.) pallide finscescens: crisso fasciaqnie alarum oblique albis.

Nilss. l. c. $-\alpha$. Sv. Zool.

$I^{\prime} a r . \gamma \cdot(f$.$) albidus undulis supra rario-$ ribus subfuscescentibus.

Nilss. 1. c. - B. Elom in Arl. IIoln. 1785 2in. Sparrman in Míuseo Carlsuriano n. 66 . Sv. Zool.

Var. i. ncer: gula balbata, plerumuque sublus albo-jumclata.

Linn. Fil. 201. 'I. liybridus. Rutenskjold in Act. Hoim. J744 181. Nilss. 1. c. $-\gamma$. Sparrin. Mus 5 . Sv. Zool.

Sv. Rackellianc, Ruslare. $N$. Bastard 'Tiur, Tiur Aarliaise.

Var. E. albo nigroque varicgatus mac:ula pectorali caudaque nigris; digitis albo hirsiltis. Sparrm. Mi1s. 65. - Nilss. I. c. - \&? Sv. Zool.

Var. 2. supra nigro cincreopuc-manulatus: collo pectore apicilutsprue peumarmu illsis.

Thunb. in Act. Holm. I8ns. r95. - Nilss. I. c. $-\delta$. Hamin. Fn. 214. Sv. Zool.

Hab. Frequenlissime in sylris ubique, presertim in beluletis; varielates rariores: $\beta$ Uplandize (D. Bl(sul). $\gamma$ Dalecaslie, f pluribus in provinciis (hybrida e m T. tetricis el f. T. urogalli), $\varepsilon$ Norrlandize (D. Törnros) et (Wernilandize (liybridie e $\mathbf{m}$. T. cetricis et f. T: lapponici). Prater cibunı T. Urogalli ova formicarun de. leclaut. Mas in slatu coeundi mensibus Martio-Apr $f$ : alleciat clamore eruuivel fraui' gol gol gol roi. deinum reluli cum cachinno $D g$ go Tjouysch! Ad venalurem pellicitur voce frau per manum clausam. Mafuiludo maris femina T. urogalli aquat l. longitudine 2 ped. 10 poll.; $f$ ponit et 5 septimanas foret ura 8.12 , ourdide luteo-alba punclis ferrugineis, Iris cxrulescens. Couda bilurca bone cognitus.
3. bonasia: ferugineseens fusco-maculatus: rectricibus cinereis nigro-punctatis, fascia intermediis 2 exceptis ante apicen lata nigra; m. gula nigra.

$f$. gula albicla, frisco maculata.

Linn S. X. 275 9. Fn. 204. Retz. Fn. 187. Nilss Orn. 158. Hamm. Fil. 217. - Pontopp N. H. 2.12g. Sv. Zool. Sir. Godmalsorre, Iljerpe. $N$. Hjerpe, Jer. pe, Norsk aferliöuc.

Yar. B. sinilis var. a $f$, sed colore dilu. tissimo ranescel.te.

Sporrm. Mns. 16. Retz. I. c. Nilss. I. e. Su. Znol. Hal. per lotam Srandunavian borealent in belulelis. var $(3$ vero in Helsingit ( $D$ Com. Stenbock) rarissime. Vescitur precipue baccis variis et amentis Bellize albx. Ova ro-ra paltide-ferruginea fusca-naculata $f$. ponit et seplimanas 3 incubat. - Longiludo circa $\mathrm{J}_{2}$ poll. Irís obscure fusca. Ungres albi, inierdum lusco-maculati. *) digilis lirsutis:

1. lapponicus (hieme: totus albus), astate: capite collogne fulvescentibus, vigromaculatis; dorso nigro ferriginco undulato; rectricibus If nigris, apice remigibusque totis albis; digitis sublus mudis.

Linn. S. N 274. 4. Fn. 303. Hamm. Fn. 2xg. T. lagopus. Retz. Fn. 185. T. cachinnans. Leem Lappl. 243 . Pon-

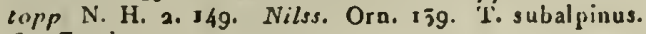
Sv. Zool.

Sr. lappsk Orre, Sü̈ripa, Skogstipa. $N$. Sloviype, Lierype, Dalrype. Lapponicis: Pichussak.

Hab in regionibus $\mathbf{S}$ candinavix septentrionalibus sub. - ipinis. Nomea ejus anliquissimun, ut speciei distincte. - D. Lathain datum conservarimus. Mas evolat rum soau cachipuanie, ad quem $f$. respondet $J a \in k, j a c k$. 

Gommx fruticum, bacex, interdum eljam insects es $c$. bum prebinl In monngsnia virunt, coülntes nellse Majo; el $f$ ura $x$ J.12 leriuginea fuscci-niaculara sub fru-

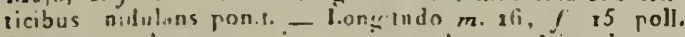
Rustrum robuslum apice incurvo obiuso live olsecure fusca. Ungares parum curvari, lusci, malgine aj.cequa

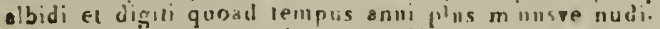

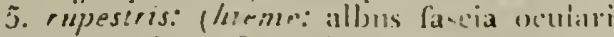

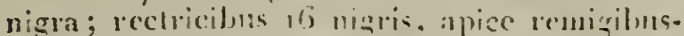

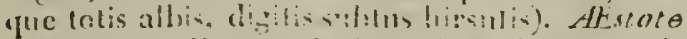

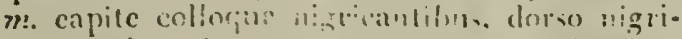

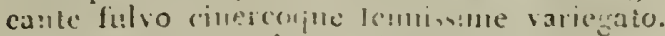

f. nigra fircios fithis allisipue.

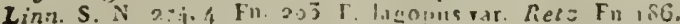

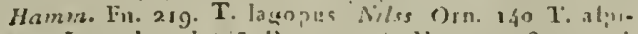

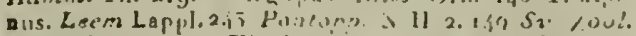

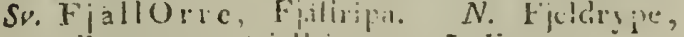
Sharcripe, Finll arw. L. Kerou.

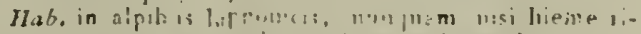

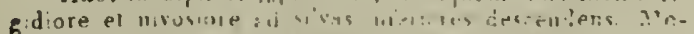

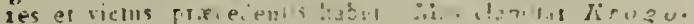

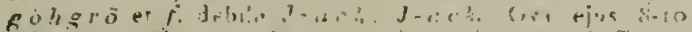

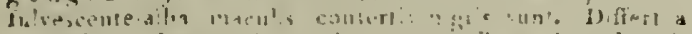

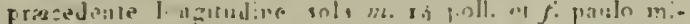

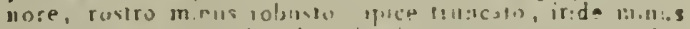

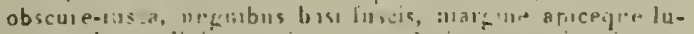

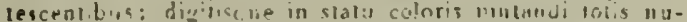

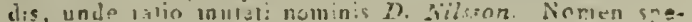

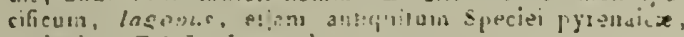

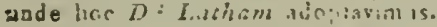

\section{TiR. 2. CONORHAMHAR,}

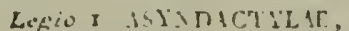

Niat. '. CUi.l lii; illlis,

G. 4. COLU.ME.t ${ }^{*}$ ), Si, Dufra,

- Ifahir fere Prrdicis, sed rosero magis conoideo,

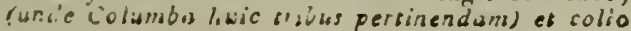

1. policilld

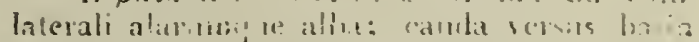

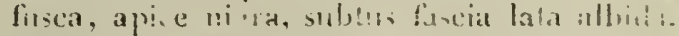

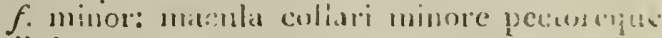
pallidione.

Linn. S N. 28, In Fn. 208 Retz. Fn. 19t, Nilss. Crn. in Hamme. Fn. $22 \mathrm{r}$. Sv loml.

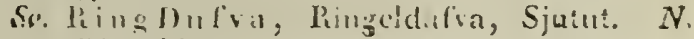
Rireglder.

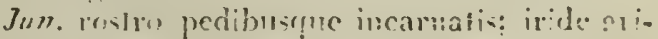
ser-iliba; colore cervicis nitente macula alsui (ril:eel.

Hal. in sylvis mon infrupillene; migratorius mense

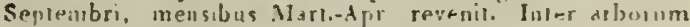
ramulus nidulans, bis nra 2 niba fmo polat at dies 20 incubas. Funclus vaccinii myruli, fruspenta raria semi.

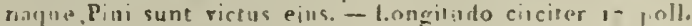
Canot cinetes carrutesells; rieniluana narium cumida

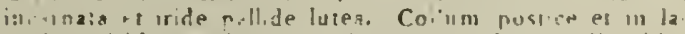

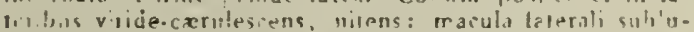

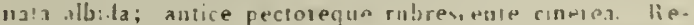
mires nislescentes; frimures magine exteliure ailo io. Peifi rubri.

2. whzs: c.emlescrus: moprgin pallinle cincreo; alis facia dupteci niera; cullel ci. nerea versus apicem nigrar, fascia subtur (1. tlerea.

Linn. S. N. 2-g. 1, In. 207. Nits. Orn. 155. Jismm. Fn, 2zo. Sv. 7.nol.

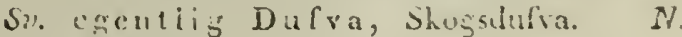
Shovelne.

pedibusque paulo brevioribus. All melicres:

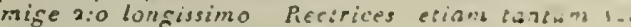

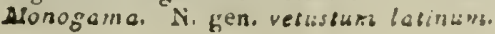


Un's, in sylvis ubique frequenter. Mligtat mense Octobri reveniells mense Martio; inlerdum vero etsi raro, in cavirate aboris hibernans inventa est. Nidulat in covis alonibus, ubi fem. ova 2 albs ponit et dies 18 foret. D.ffelt a priore magniludine minore, longitudine tanturn 13 poll; capite, collo, dorso et uropygio cinerasceatibus irideque fusca.

3. livia: crrulescens: membrana nariun allescente; mopygio albo; fasciis 2 alarum a picerpue cattis tiiglis.

Linn. S. N. ed. Gmal. -60.2 . C. domestic B. Retz Fn. rgo. C. cenas 3. Nilss. Oill 296i. C. dónestica fera. Pontopp. N. H. 2.115. Su. Zool.

S2\%. blägläusande-Dufva. $N$. Vildduc.

Hab. Norregix pracipue in Ryefylke, ubj nidulat in rupibus, raro alibi. Ab hac specie et anrecedente $\mathrm{C}_{0-}$ Jumba doinestica plurimx originem suam ducunt. Diffelt a pracedentibus, prater cliaracteres indicatos, magnitudine misore 1. longitudine tantum 12 poll.

\section{Leg. 2. TISYNDACTYLAE,}

Col. 1. INTEGripostries:

Nac. 4. CORVIDES,

\section{c. 5. CORVUS *), Sv. Korp.}

") cauda mediocri:

1. $\operatorname{cor} a x$ : ater supra purpureo, subtus viridescente-nitens: cauda cuneato-rotundata; tibiis longitudine rostri.

-) Hab. gener. Corpus ovaie: alis cauta brevioribus, remigibas: primo mediocri, a sextu longiore, 3 fere longitudine quinti vel quarti ut longissimi; pedibusque mediocribus validiusculis anbulatoriis: cibiis digilo incermedio longioribus. N. gell "lacinum Gesneri; sed apud Plinium nomen iebthyologicum.
Linn. S. N. 135. 2 Fn. 85. Retz. Fu. 10. Nilss. Orn. 35 Scandinavisk Fauna a Del. I Band. S. n17.n. 1. Hlamm. Fin. \&g. Sv. Zool.

Sv. Siart Korp, Rawn. N. Ravnekrage, Ravnen.

Hab. Sere per $1014 \mathrm{~m}$ Scandinaviam frequenter; maximus inter Conorhamplas nostratas, longiludine circe a pedum. Nidulans in arboribus excelsis et speciebus mon. lium jam sub finen hiemis, primo vere fem. ova 4.5 sordide viridescentia, fusco-maculala ponit et 20 dies incubar. Victitat animalculis diversis to frugibus, precipue tanirn catlaveribus, qua odoratu suo subriii e longinquo indayal. Sub volatu alrissimo crocitat Kraak, kraak vel Kruck kruck \& in lempore coëundi $K l o n g$, Klong. Docilis est ftjam ad loquendum et xtatem ad ron anuos ustuue producere dicitur. - Colore atro bene distinctus: lris tantum extus albida intus griseo-fusca; fem. mare parum minor

2. corone: ater supra violaceo-niteus; rostro 'tiłiis Jreviore; fronte plumosa; cauda jotundata.

Linn. S. N. 155. 3. Fu. 86. Retz. Fu. 4r. Nilss. Ora. 66. Fn, 120 2. Hazmin Fu. 92. Sv. Zool.

Sv. B I a Korp, Rauk. N. Blaa Kaage.

Vor. B. ater macula gulari alba.

Sparrm. Mus. 2. C. clericus. Nilss. 1. c.

Hab. in Srecia rarior, insula Gottland excepla, ubi in regione australi, (ut et in insula Oland) sat copiose, pressrtim in parceriis Grötulingbo \& Wamblingbo, invenitur, var. $\beta$ rarissime in Wermlandia (D. Sparrman); mizualls. Minres et victus ejus fere pracedentis, a quo lainen diffest magnitudine ninore, lougitudine 1 antum 20 poll. Colore magis crerulescente el tibiis longioribus, et a sequente fronte plumosa diversus est.

3. frugrlegus: ater supr'a purpurco-nitens; rostro longitudiue tibiarum subrecto acuto, fronte calva; cauda rotuadata. 

Limn, S, N, I,5, 1. Fn. 8\%, Retz. Fn, 42, s, Ritiss, Orn, 57 I'I. I.24 4 . Sv Znol

S7: Rokhor p, Ritia, Raka.

.lun. fromle p! tonosia.

Hab, in Srecite altalra!t sat frequens; in fine Sept. migrans revellim ad finsm hiem, In arbmibus excelsis muse pracedeutis gragatim nidatic: al lase perpeluo rociferat $G a n r b, G a r r b$ ! Fem, iva $\mathbf{j}^{-5}$ glanrescenlad tuicramacula a ponil bis pet amumu Victis ilam ac

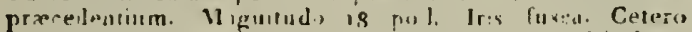
bavi rosiri calva, rugnsa, a ho grisescense in adulcis b.ne cognita.

4. cornix: cincecus: capite, j:igulo, alis casulaug!t: uigrois

Linn. S. N 156. 5. Fn. 88. Retz. Fr. 亿2. Vilss. Orn. јs.

Fu. 122. i. Ilamm. Fu, gn. Siv. \%oul.

Sv. K vitka Kor p. N. Krägr, lítake.

Vis. \%. infus allsidles.

Rulb, Av. p. 15\%. Cornix al, sranıliana. Nilss. 1. c. $-\alpha$. llal, in scandinavi, tola vulgaussinatis, var. $\beta$ iamen

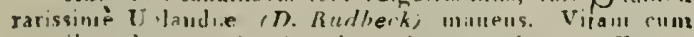

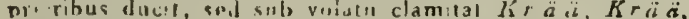
imerdum Grah! Punit in mensibus Apr. Majo ova 4-6 pallide virulie lusco-nacalind - Longirudo 13 poll. Rosirum, capur, jugstum al medium pecloris, alie, podes candaque nigra, crlerd cilteres.

5. mibedule: niscl sublus nigrn-ciun'ells: capile, cervice, lateribusgue capitis el colli inc'atis.

Linn, S. N. 156. 6. Fn. 89. Retz. Fn. 45. Nilss. Orm. 39. Fis. 27. 5. $11 \mathrm{nmm}$. Fn 21. Sv. Zuol.

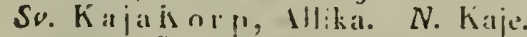

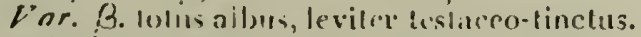
It.sb. ubi jue non infrequens gregralim in iedibus antiqiis, [rmetten tuiribas, ubi hiberuane tt nidulanlur, ralo in splvis var. ") rarissmua (D. Pavkull). Sonum Ja $k$. Krik, $\ddot{a}<k$ ! perpetuo edunt. liem. ova $4-f i$ visidescontia inaculis striisque fuscis ponit, Docilis al li, quenilum est. Insecis, vermes, nra orimm aliarum, fru-

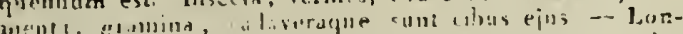

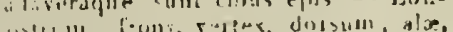
fieclo 15 ['ull.

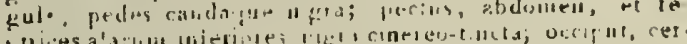

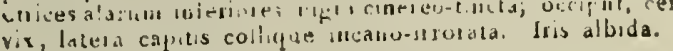

*) cauda ninngata:

(i. pisa: niger crrmblemente vilidescente-

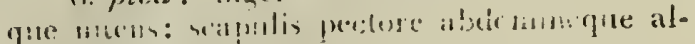

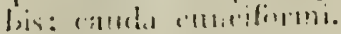

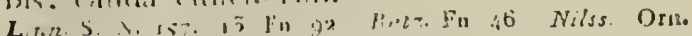

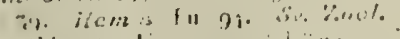

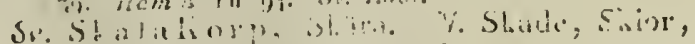

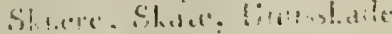

J gr.

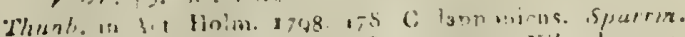

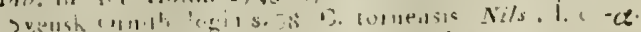

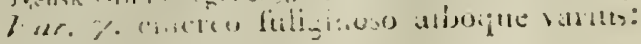

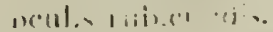

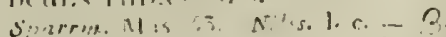

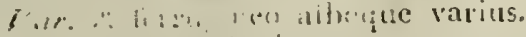

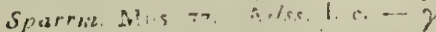

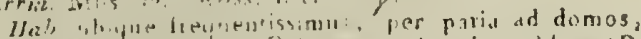

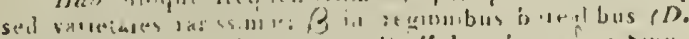

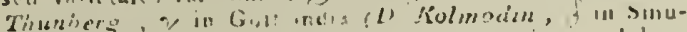

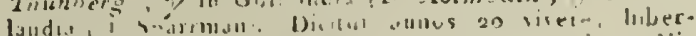

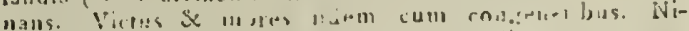

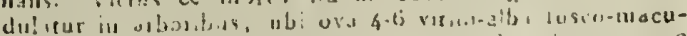

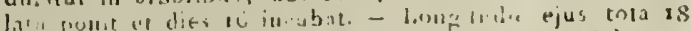

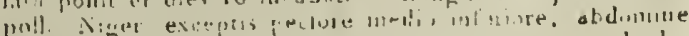

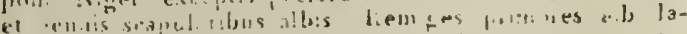

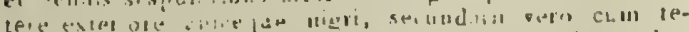

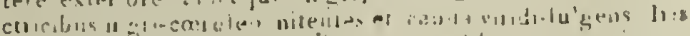

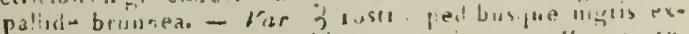

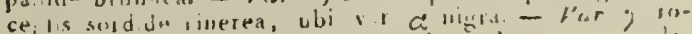

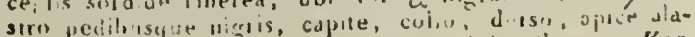

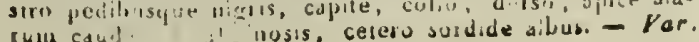



$\delta$ capite, collo, pactore medio superne et marcinibus pennarum fermginescentusus; celeso albidus, pedibus nigroscentibus exceptis.

\section{G. 6. CRACTES ${ }^{*}$ ), Sv. Skrika.}

1. infunstus: rubrescente cinereus, capitc fusco; tcclricibus alarum, uopygio, crisis caudague cuneato-roturdati filvo. Perrugincis; rectricibus internnediis 2 cincreis.

Linn. S. N. 158 25. Lanius - Fn. 95. Retz Fn. 47. Sparron. MI s. 76. Niiss. Oru. 41. Corvus - Fn 156 s, G.ırusJus. Ilamm. Fu. 95. Corvus. Sv, ool.

Sv. OlycksSkrika, Olyckstigel, Enskrika, Lafskrika, Flarshrika, Trllliuxa, Rïiltjuxa, Lapjskata. N. Lavskriig, Lavskıjke, Rölinluc, Rendikjöve, Reudijuxa, Giertiutsfingl, Ulykiesfiugl.

Hab. in nemorosis Lavponixe, Jemilindise et Dalrarlie; ram tamen in U!n! ndia et adhuc ratin in Golllandia obvenit. Slanitat s-kruih, s-kruilh. In abboribus puetis nidula:s, fem. 5.0 ova cinerdseatia maculis obscuris mense Majo ponit. - Lasngilud, Ir poll. $C_{\text {a- }}$ put obscuse fis: uin sut -rin tuin :ortro nisto: fromle in testareo pallidiure fistescemte; collo, dorso pectormque rufo-griseis. Ablome, hypochondria ol crisanm magis rufescentia Remigen tusci bis tirrugilla; latese exteriore cinerei; tectic bus po a ribus proximis ferrugineis - pice fuscis. Cauda fulru-fenuginea: recricibus inter-

-) Hab. gener. fera Consit; rell copice proportionoliter

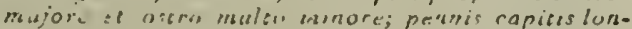

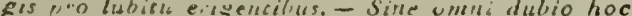
Gevis disbin'sic 2 ; sed noinen generscum fastulus

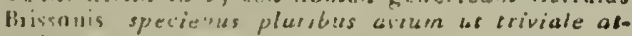
tributum, intel.tm est, unile Gractes, c roce heviast, s (clumatur), asdoptavinus. mediis 2 nbscurius cinereis tectriclusque inferionibus piallice rufis

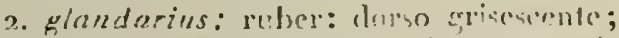

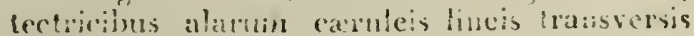
allis nigrisplec.

Linn. S. 1. 15ti. ?. Fu, go. Retz. Fn. 4h. Sv. Zool. 26. Corvus Nilss. Orn. 34. Lanius. - Fr. 17y. Galsulus. So. AllouSkitha, Vïskitial, Kormakiki,

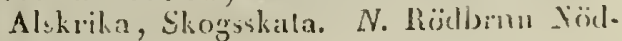
doskricte.

Hab. nibiut liequens, autumno hiemeque praser-

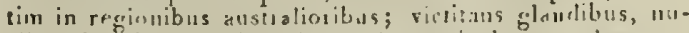
cibus fiuchionsque, interden etian aviculas et coleoplera devorat. Sidnduriur in alburibus, ova 5-7 soldide viride. scentia fusco-maculata porens. Sonat sub rulatu $G a ̈ a r k$,

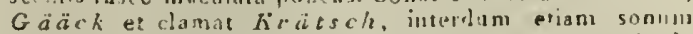
exprmere audiur similem Mlarkolfus. - Longitudo I $\frac{1}{2}$ poll. Fruns aiba maculis longindinalibus maculaque ab sugulo oris eblunga descendente nișris. Jris guiseoalba. Dorsum prisuscins; genda. crissurn, uropygium fe-

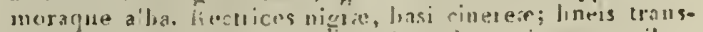
rersis nigris, extenioritus 2 fuscis, subtus cinerascentibus. Pedes wbscure iniasm il.

\section{G. 7. CAR,YOCATACTES *), Sv, Nötksücks,}

1. guttatus: fusctus albo-tyuttatus: alis cau-

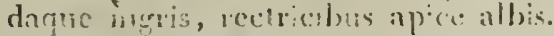

Lizr. S. N. 157, 12 Fu. 9x. Jeet3. Fu. 45 Cinruis Cary-

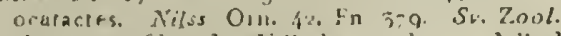

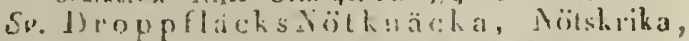
jöivacka. N. Sireicel hribleskrige.

") Hat genur. medius infer Corvos et Picos; pedillue

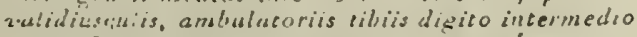

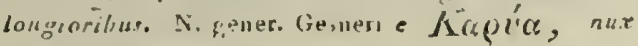
et $\operatorname{Lix}\{\langle\dot{x} \tau\})$, fractor. 

Hab. "bique vulgaris ut antecedens. Vicsitat pelate

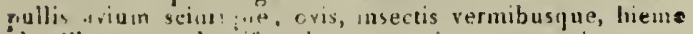

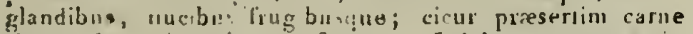
alitur. In curis ashoru ben ova 5-b lureo-grisescentia pouit In a leit s ramsilosinn sndens injucunde vocile3otur: $g r a ̈, g r a ̈, g r c i$ - Longitudo ejus 13 poll. Cospus fuscum maculis jarvis nvatis a!bis, pileo uropygioque iminaculatis exceptis, et macula majore inter oculos

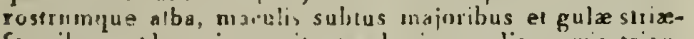
formibus. Alac vigre nitentes basi maculis parvis trianfularibus albs: renigibus medis latere interiors albis epice mbo marginati. fiostlum caudague "lgra: rectricibus apice, iuterusedis tamen minus abbis; tectricibus super. migris, infer. albis. Crissum album.

\section{G. 8. CORACIA'S *), Sv. Kråkling.}

r. garrula: viridescenti cierulea: dorso rubro; remigibus nigris; $\boldsymbol{m}$. rectrice extimo a jice nigro.

$f$. colore sordidiore: rectricis apicc extimat haisd nigro.

Liฯn. S. N. 159. 1. Fn. 94. Retz. Fn. 48. Nilss Orn. 43. Fit. 15r. x. Hamen. in. 96.-Hellenius in Act. Ho!m. 1787.308 . Sv. Zool.

Sr. pla delerkrikling, Blihhrâka, Sparskkrika. N. Kuivsnabel, Blaakrake, Blaakıagc.

IIab. fere ubique, migrat vero in mense Sept reveniens Mijo. Rants, in ectis, vermbus frughusyue ve. scitur. ividulatur in cavis atborum ora $\mathbf{3} 7$ albo unmsculata

-) Hab. Gener approximat Craclis; sed cauda ininus

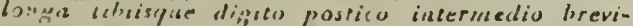
orlews; wlis longis: remige primo, quam secunds" langisinas, paulo breviore. Nour. geaser. Liauxi a dióplis, Corrus. cular ponens, qux dies $3 n$ mas ef femias alterratim incubant. r.lunita liach! et Kachi! - Longitudo

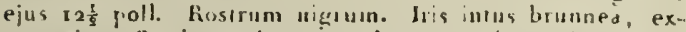
las grisea. Regrio oculoruni nuda; verucis molis in turn. pole cuéundi clevalis. Carilt, nucha, pectus el lectuices alarum inajores colore viride conte cxru'eo, nallidiuse vero abdominis, crissi et In lricum alatum infertorum. Uropycium el hasis alduin nitidisime-rerulea Cervix, dorsum et rens:ges intimi hepaticerubu, extimi supra nigri, basi pallide piridescente-cesulpi, ublus latere inmeriore aptendidissime cactulei Rectrices basi o' scurecrevulex versus apicem virdescentes; interuned is 2 ubscurce-viridibus. Pedes lutescemles; unguibus nigris.

\section{Nat. 5. STURNIDES,}

\section{G. 9. STURNUS *), Sv. Stare.}

r. mlgaris: niger, purpureviride ca. rulengue nitens: maculis parvis testaceo albis adypersis.

Liun S. N. 290. I. Fn Su. 213 . Retz. Tn. 199 Nilss. Orn. 121. Fn. 573. T, Hamm Fn. 220 - Sv. Zoul.

Sv. allmän Statr - N. Star.

l ar. O.eolore plis minuve cinerascenle, albar rel isabs!lina. Milsi. /. c.

juniur: matus nilcus: 10-110 nigin sı butus furo; maculis superaic columblatis la idc-

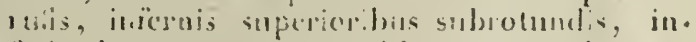

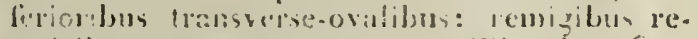

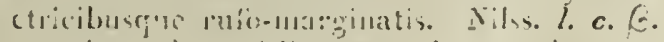

prollus: fissco-finigandusus, inmaculatus: re-

-) llub. gener. Caryocalacli approvimat: sed digitis exteriolibus andacis baci unitis; alisyue longus: remigibus a et 3 longissimis. N. gren. latinum. 
migibus ferruginco-maculatis; subtus palfikle-

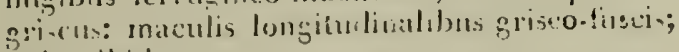
molla allicita.

IIa $\therefore$ ubique frequenter. Migrans mense Sept. jam

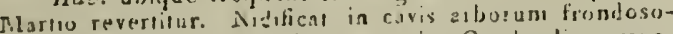

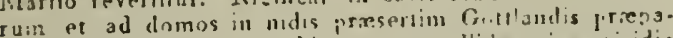
ratis, skânkor dictis, ubi 4.7 ova pallicle grismo-vitidia ponil el dies if incubat. Inseta, vermes, inferdum eziam semins varia victus rjus. P'ellicit gro äck! et strepiter non insure cantillat Fachle munillescens, cantus $\mathrm{va}$ rins, sibilus et sermones hominum imilare docilis est.-

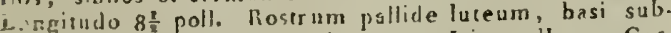
tus albillu, pust mortem ragrescens. Iris aveilna. Cr. pus subers ininaculalum: crisso excepro, cujus pentus IL:do.finbilitis Keniges latere cxteriore nori lentio if: pallide lespacen-marginati, interiore cusci. Cauda fei zejualis. Pedes luterg-ricarnati.

\section{Nat. 6. FRIXGIIT EIDFS.}

G. I0. LOXIA *), Sv. Korsnäbb.

8. mojor: sordide-olimarca: ylala lnter.

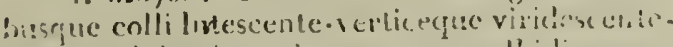

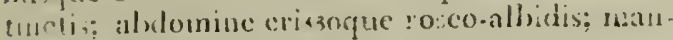
-librlit inferiore valcle berevione.

f. viridescente-cinerea manulis brumesecnliburs: gula colloque gristscentibus brisisco. tinctis; retero subtus virescente-cinerca: crisso litescentc; ablominc tectricibusque inferiomtus caula allsidis.

-) Hab. gun. Corpus erassum: capice magnn collogile Brevi; alia mediorres, remige 1:u longissionn.

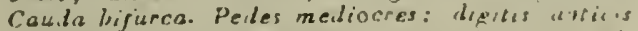
fere divisis, ungue postico antiris longsiare. N pewer. e verbo greco dustis, obliquss.
Rublinck aves pin Ed. De Geerinnat. Inn. Lovia m jun.

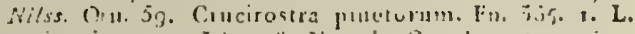

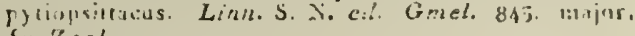
So Zool.

So. stor korsinilit.

anallosps me colere: plas minusse minials tretricibusguc infericribus abidis: maculie oblomsis gy isero-firacis.

Junior: fere $f$ similis.

Hol. in pinetis ( $D$. Rudberk: Inter ranulus pino rum, cujus alucque semiribus victiat, nidulatur. ub fern. ova 4 mence Majo noul. Pe!licit jopjup/of!

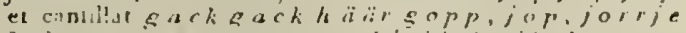

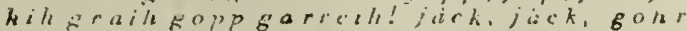
gohreh! - longialds 7 i oll Aostum sobisschen ni.

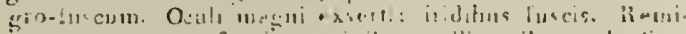

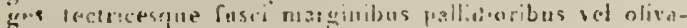
cers vel albidis, sublus ria!lide-wisei. Trectices alanum

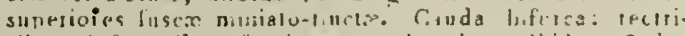

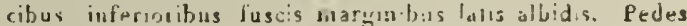
incernato-fusci.

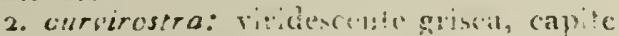

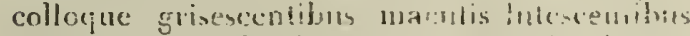

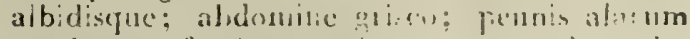

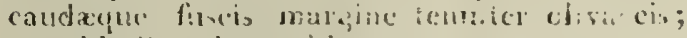
mand.hulis subrequalim.s.

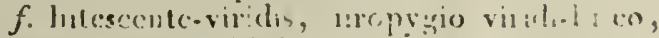
sublus gritea viricli luteo tibrlit.

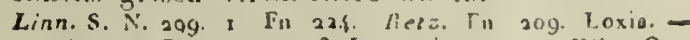
IIamm. In. I 50 250. I.. co virnura. - Vilss. Oin. 6o. Curvirusira abletina Fin. Jü. 2 - .52. Zno:

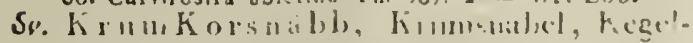

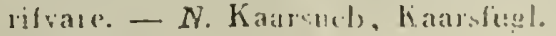

annuosns m. colore plus minusuc mition's Junior: fere ut in firn.

Bab. ill abielig non infreyuens. Nidulatur in ams: 
lis pinnrmm ora 4 jempridem mense Jan-Fehr. grisescenteaba nonens, gure dies 14 irrubat Non sulum serin: bus abiefis, sed rtiam barcis valtis vescitur. Irel-

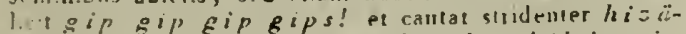
razirt tsys!dons düng histhisthihi. eip gilerfgip; dihnifn dihuija! gagaga; Longitudo $6 \frac{1}{2}$ poll. Finstrum mius robustum fuscnin. Iris brunned. Tectrices alatum el scapuldres vilidescentes. Urupygium fiavum. Cauda bifurea: rectricibus subtus pallidegriseis Pedes luzci.

\section{G. IJ. CORYTHUS "), Sv. Håıdnäbb. .}

1. enuclencor: aurantiacus: dorso urnpvginque obscuroribus; fascia lineari duplici alirım albis; cauda nigricante.

f. viridesecns: maculis brumneis, dorso obscrurine; capite atrantiaco-linclo.

Linn. S. N. 209. 5. Fn. 225. Retz. Fil. 21x. Hamm Fn. 2\%5. Zebcerscedes lidpska resa 1. Del sid. $24 \hat{1}$. Nilss. Oru Gr. Loxia - Fn. 560 T. - sv. Zool.

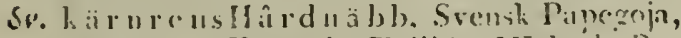
Naltraka, Kattrak, Tallhit, Milktel, Pa:ristigel.-N. Noisk pape:roje, Fongelbirl. annuosus m. plus minusve chirmesinus: pen-

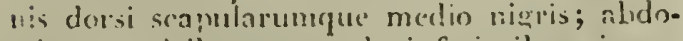
mine tectricibusque candx inferioribus cincrasciutiluss.

junior: similis fere fem.

l'ar. G. alba: capific, collo supra urnjygiopac lafescentibus; ábluminc confiäie fusco.

- llat gener. Inrie; sed tibis dizito merlio antico vix brevioriós, alis mobiocribus remigibus a. 3 lunnissilidis; ribda paulu latigure sub.bijurca. ג. sener e húvvús, armalus. maculato; alis nigricantibus albo-macrilatis slrialisqur; caldal nigricalule. Nilis. l. cevevr. a.

Halh. placsestm in reginubus septentrionstibus; rar. B tamen rasssime, D. Paykull in Lapponiagrectuse in pineris ad baseus'ranulurum nidificat el bi mensa Junio fem. $\mathbf{i}-4$ ova pallide-sluucuscentia : punctis m.jint bils minoribuste niglis, brunnescantubus grisescantibus jue, interdom albidıs, ponit. Seml:t:bus pini, abietis et bactcarmun diversarum vicritat, facillime mansuescens etiaun fiamen. tum avinacellum, grana divelsa, Rer. guslat; figus tainen in hac statu ei necessum eit. Nortil jucunde rantillat $\rightarrow$ Longitudo fere 8 pnll. Finstrum luscum subros bass pallide incalnatum. Lora fusca. Remiunes nigricantes ma:gine exteriore tenue allidi: tectricibus majuribus uraque serie nigricantibus apice albo-marginatis. Rectrices margine lenue pallidie. Pedes fusco-nigricantes.

G. 12. PYRRHULA *), $S_{v}$, Domherre.

I. rubicrlla: capile, alis caudaque nigris; dorso cincreo; uropygio alba; subtus rubra. $f$. dorio cirsereo, liusco-tincto; subtus cinerea: pidibus fuscis.

Linn. S. N. 500, 4. Fn. 225. Resz. Fn. 212. Hamm. Fn. 257. Nilss. Orn. 6j. Loxia Pyrrhula Fn. 35, - Sv. Zool.

Sv. rididett Domherre. - N. Domherre, Dompap.

Var. 3. **) alba: capite supra rubro-tinclo; dorso maculis raris lateribusque caudx

-) $\mathrm{Hab}$ gener. Corylhi approximat, sed rostro valde minori; stabura corporis breviori; cauda rosun-

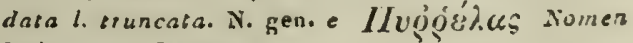
hujus avis, Gaza.

-1) Per comparationem hujus varietatis in Museo Regni cuin P. rubicilla et Corytho enucleatore, niblum dubium est, quin D. Nilsson ei locum veriom huic dedisset. 

discoscenlib:ı; remieribus 4, 5 exlus nipris; s'lins palliale-11,inianiat.

Nilss 1. r val. a Sparrme. Mus. 17. Jonia Aaminzo.

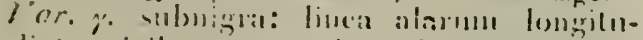
disoli learicibusque cande inferioribus albis. i:in. l. c. zor. B.

fur. i. ia!di nimlia.

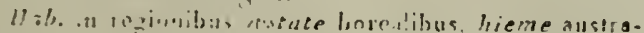

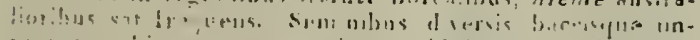

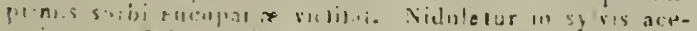

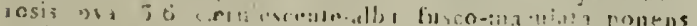

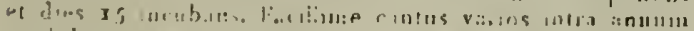

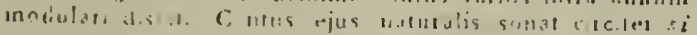

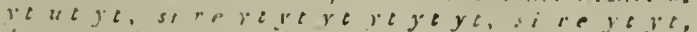

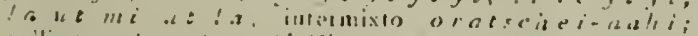

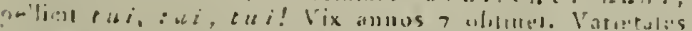

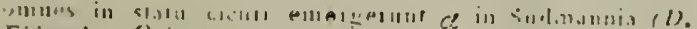

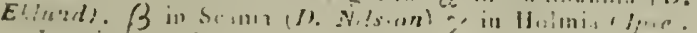

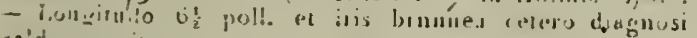
a'd r...snia.

G. 13. PYPGITA *), Sv. Tjocknaisb.

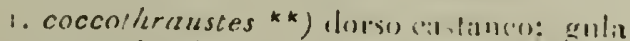

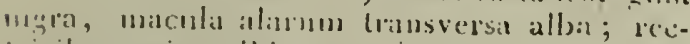
toiribus apice: alf,is.

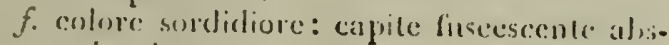
ifue: "zlla ni:;ril.

Linn. S. N. 239. 2. Fa 223. Rie:s. Fn. 21n. Nilss. Oin i2. Loxia. - Ln. Ji7. J. Fillugila. -- Sv. Zuol.

-) Hish. granar. Furrlulas afproximat; sed petilius al.sque breivilus: remighlus 2 et j longussionis. a. cen.e I/Jugite, nomen grecum spesieidu. mecture hujus gelleris.

") Anne lace sprcirs rum sequente nt, rostrum ma. gie crassum. latoconcideuin, circullucurca conte6412 propriuin genus sib.
Sv. Frïknäck Tocknal,b, Slouknack.

Junior: yula firsco-incarnala: lalcribus colli, hypochondris pectoregue incarnato-sinescis,

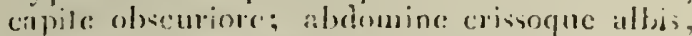

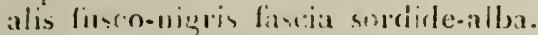

IIab. in Golllandia at provinciis anstraliosibus haud

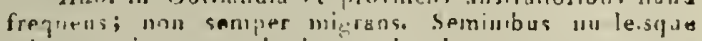

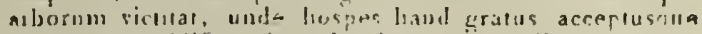

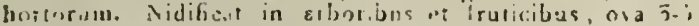

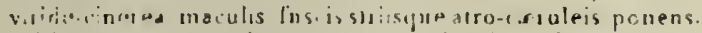

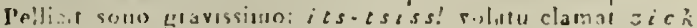

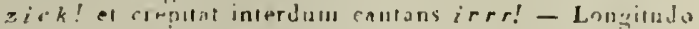
7 pull. Rustium crassissimum plunbeum, subtus versus basin a!bum, hisme pollide iucituatun epice ugtirante. lris s'bida. Caput el tectrices superiotes calda lupes-

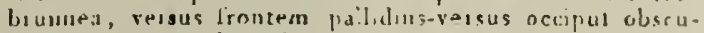

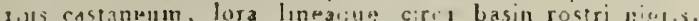

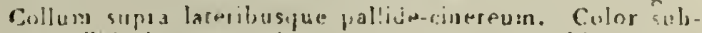

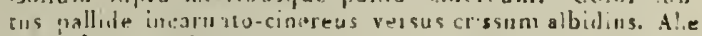

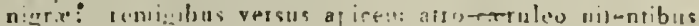

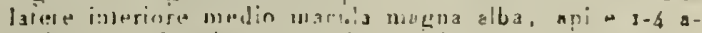

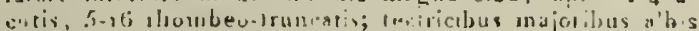

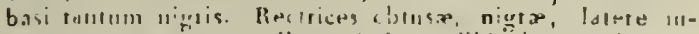
leriure ju:sia apicem albx. Petles pallide incarnati.

2. chloris: nlivacea cinceen immixio; remigibus primoribus extus rectreibuspuc f́la. tcialibus lasio luteis.

f. srisco-finda: nroprgin lulen viridi: sub. 111- lisment:ineres: pecture abdomineque viride. llive illumixlo.

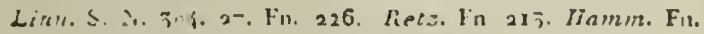
1. 5n. 278 Tislss. Oin 64. Laxia. - Fn. 330. 4. Sw. Ziol.

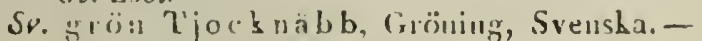
N. Sicenthe, Sivensh lruisk.

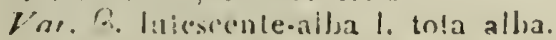

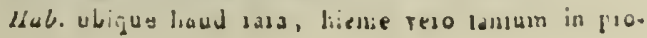



rinciis ansiralioribus; var. $\beta$ in Scania rarissima, Seminibus variis oleosis baccisque juniperi vescitur, ounquam inseclis, hieme etiam gemmis piacipue quercus al salicis. Nidificat non alte a terra ill alboribus vel fruticibus, ova 5.6 albida, puncris naucioribus fuscis al violaceis ponens. Cantillat non injucunde, ciom tat sub volatu $j a c k, j a ̈ c k$, jäck! et pellicil: schivoinz! - Longlludo 6 poll. Rostrum incarnatuon subtus pallidins. Iris tusca. Frons, supercilia er uropygium flavo-viridia. Subeus tlavo-viridis collo pectoreque lamen nugis viridlibus abdomineque magis flavo, crisso tectrichusijue cauda inferioribing albis Aaro-tiuclis. Pedes fusen-ncarnati: ungubus fuscis.

3. dornestica: ('ippile supla carulescentecincreo, dorao biunnercente marulis uigris; remigibus rectricibusque obscure fuscis: folscia alarmm solitaria alloa.

f. capite supra rulescente-cinereo; linca su. prin oculos postice litiore fastiaque aliurum ochuaceo-albis: suhlus soidide griven-albu.

Linn. S. N. 325. 36. Fn. 2\{2. Retz. Fil. s2s. Nils.s. Orn. 68. Fu. 521. 2. Hamm. Fu I. 52 249. Fuingiila. Sv. Znol.

Sv. husTiocknäbb. Husink, Grisparf, Gråspink, Grânamb, Tälling. - N. Huus. spurv, IHuskild, Granspurv.

Par. B. f. fasciis alamm 2 albis oblicfuis, superine majore; remigibus rectricibusque tenuiter albo-marginatis.

Nilss. 1. c. '.

Var. $\dot{\gamma}$. similis val $\alpha$ sed remigibus primoribus albis.

J'ar. $f$ sublus caput collumque sordidealba; fascia alarum superiore alloa, allera lutea; cauda fusca : rectricibus lateralibus di. midiato oblirgue albis.

Nilss. 1. c. E.
Var. $\varepsilon$. supra pallide fuseo-ferruginescens, subtus et randa albida sordide lulescente lin. cta: rostron pedibusque aibis.

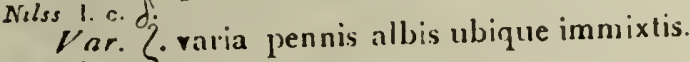
Nils. I c. $\gamma$.

$V$ ar. \% tola sordide alba.

Nilss. 1. c. $\beta$.

F'ar. i). tola allia.

sparim Mus a ro. Frincilila candida.

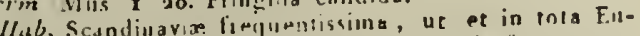

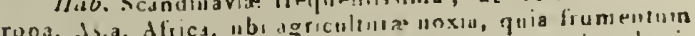
est cibs ejus pionrius. Sub teclis, in musis allonsibusyul jam mens.bus Mall tuidffert; fem. ova pletumgut 6 viridescantealba ponens, dies 14 cum mareaterque 6 viridescunte-alba ponens,

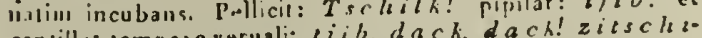
cantillat tempole vernali: $b j i b$, d $a r k$, dack! zitsch asclit! Varielates invente suni (S in Uplatalia i D. Thunlerg) ig Holwia: (Ipse) fo et $\zeta$ in icania ( $D$. Nilsson . $\varepsilon$ el $y$ ill Uplandia (D. de Paykull) g Osirngrulhia $D .5$ pariel ma) - Longitudo fere 6 poll. Fortrum supra fuscum man). Longitudo lere bulis lusco-incarnatum. Iris

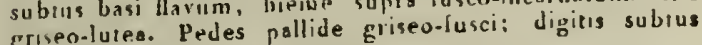
lillesceuli,bus.

4. montana: capite colloque rufo-fnscis; dorso ferrugiueo-niçroque maculato; alis caı. daque obscure fuscis: fasciis 2 alarum albis. $f$. capite pallidiore, maculis gula laterum. que capitis nigris minoribus.

Linn. S. N. 324. 37. Tn. 2.13. Retz Fn. 229. Nilss. Oro. 63. Fn. 327. 3. Hlamm. Fn. 247 . Sv Zool.

Su. bergs'liockuabb, Skorssparf, 'Trädspaif, Fälsparf, I'ilspart, Tysksparf. N. Oikeul.

Var. B. ponuis albis intermixtis.

$V$ iar. $\gamma$. fiere tota alba. 
IIab. sat frequens in hortic squicetis ef fruticelig. whi in civilstibus asborun non a!je a terra nidificat, 5-6 nva fliseicentia brunseo.marmoraln ponens, qux m. et f. alipsualim incubent. Insects, larvx, frumellfum suminaqua varia victus ejus, mie gralus sit in hortis insecta larvasqua unxia dovorani. Pariplales tamen rarissinte ubraliant. Criat zielziob! ziel,! at cantillat ziebzarrzarrzwohr! - Lungiludo st poll. Rn-

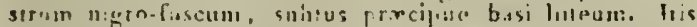

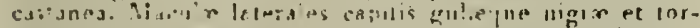
glos circs coliun albus in cerime interruplus. Pedessordide ilfcuinali

\section{G. Lf. FRINGiLLA *), Sv. Fink.}

3. crelets: capile sinerenecaruleo, fronte: nizro: dorso scapulisque castaneis; mopvigio luter-viridi, alis nigris fusoiis a obliquis alliv; rectricibus nigris, extimis a ohlifyte allsi.

f. cipite collocine stipra grisio-funcis: late.

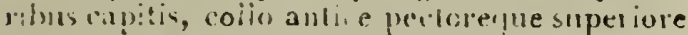
mo-cinereic, cetern siblins aibida.

Linn. S. N. îs. 3. Fn. 2iz. lięz F:1. 220 . Nilss. Orn. 6. Fn. 352, 5. Zetcerst. It, 1. 170. MIamu/n, Fn. 2,2 Su. Znol.

So. ens a m Fink, Bifink, liamptatting. N. !rufinke, !nslinke.

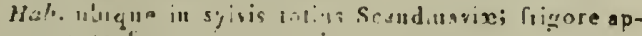

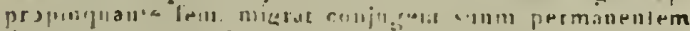

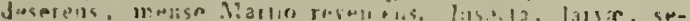

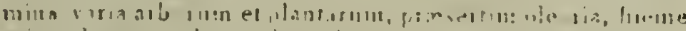

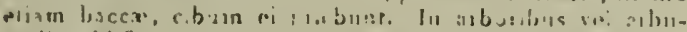

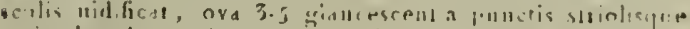

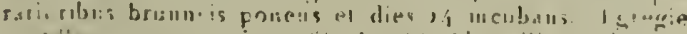
call alls, supper rial: fint, fink! pelicil: jerk, jack, juck! th anke jusnulalumen coli zinzilulat

-) Ilsbit gener. ferc proredencis sed forma rustri doverius. Ni.ienter. lubiubme esto erif. erif! - I.ongitudo 6 t poll. Fionrum tempore

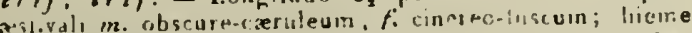

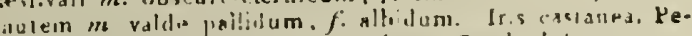
des incasil ti : digilis obscurioribis. Couria biturca.

2. nivalis: sulpra firscia ca!jle collocine

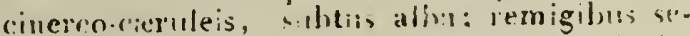

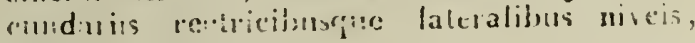
medis nigris.

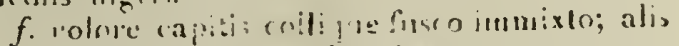

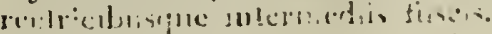

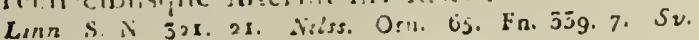
7.ool.

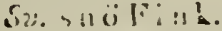

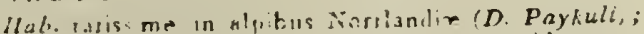

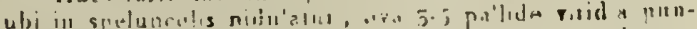

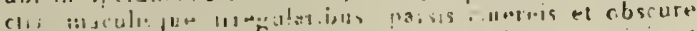

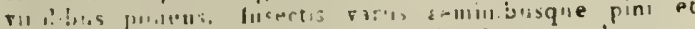

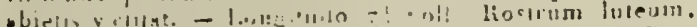

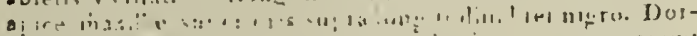

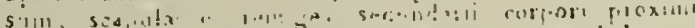

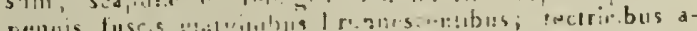

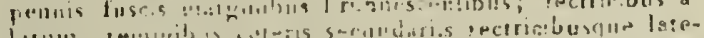

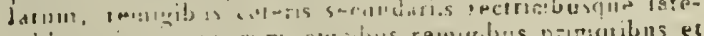

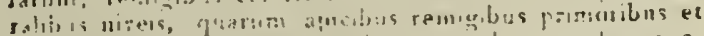

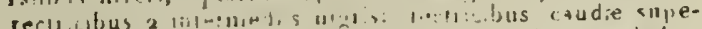

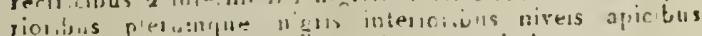

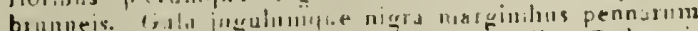

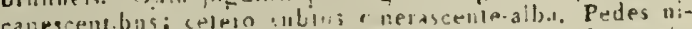

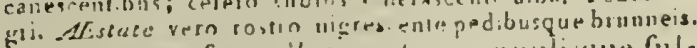

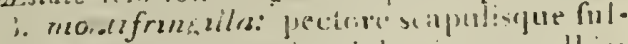

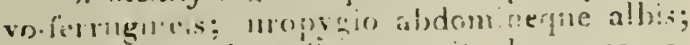
bi if ahrmm sublus Hara; caphle dorsoque an. teriore li í:

f. elpile dorsorue rufescente-cincreis; natulis nigris.

Linn. S. ‥jis. \{. Fu 25j. Rerz Fn. 221. Nilss On. 66. I: 5j5. 0. Zebferis. It. 244. Hemm. Fis 24) Sv. Zuol. 

S2. BergFink, Norrquint. - N. Quxker, Bogfinkens Huemuge.

Far. 3. capite ut in fem. sed fascia supcriore aliluus cun scapulis confluente rufoferruginea.

Hah, ia sylvis boreslibus a Norrlandia ad Lapnoniam usque hieme interdum in provinc is ausiralinribus obviens; var. 3 in IVeotroboltmia (D. Paykull). Inter ramul is pini vel abielis prnpe trunce un a torld 8 ped. alte ndifical nense Jandi, ova 5 viridescantio maculis punctisque iufis ponens. Cantillat munss suave et pellicit: $j a c k$ jack jack back quä ä $c k$ ! - Longiludo $6 \frac{2}{y}$ poll liostrum carulescens ad apicen nigrum. Iris linsca. Fascie alarinn dise aibe. quarum infenior angustior rufu-tincla Uropveium album Cauda bifurca nigra: rectricibus margine exteriore in maxima paste albidis. Pedes luscoincainati.

\section{G. 15. SPERMOLOGA *), Sv. Fiöfink.}

1. Iulensis: **) cinerascens maculis fiuscis; lateribus capitis verticectue obscur inribss; peclore, scapulis, firciarque alarum rufescentibus; gula abilomineque albis.

") Hlabit. gener. fere Pyrgita, sed forma rostri diversus. Nomen Aristutelis, e Iteplloícyos, seminilegus.

-) In sententiam Di. Nilsson alide non pousumus, hanc speciein valsetate fringillis $m$ inturing llie esse; accurasa neinper lli Rudbeck depictione aviun, et differentile cum rossro arustore, magnitu. aine minure. characteribus dengonosess exceptis, de existertia ot distincbione huju, avis, yuzmvis rarisstmi, persunit sumns. Sipecimen st museo Dit Paykull sub hoc numine priestans certissine variesas est precedentis, et opinione:, Di. Nildion duxit.
Litn. S. N. 31s. 5. Fn. 254 . Retz. Fn 222 c. hig 2.

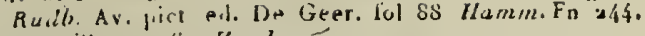
Fringilta - sv Zool.

S2. L,ile å Fröfink. - N. Lulensiak Finke. Hab. in Westrobutrnia et in eaden laritudinis gsadu Norvegice sat racus, manens. Senumibus variis oleariis salinis fue. praseslim tamen plantarum nrdinis naturalis

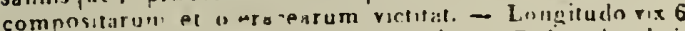
poll. Iris brannescens linstrum nigricans. Pedes plumbei. 2. spinus: viridenceus macalis nigrescen. tibus: slria onulari slyisque alarun 2 luleis, alis candiafle nigris; $m$. cappillitio gudarue ni-uis; subius !!tic'il:

$f$."ipice nizro-maculato, subtus ripidescente-illlu: gila all,ic!it.

Linn $S$ N 522 25 Tu. 257 . Rets Fn. 224. Nilss Orn.

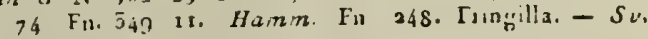
Zuo!

S2! l'örn F riilink, Gröinsicka. - N. Sini,k. l'sr. 3. lit rovans maculis supra rariorib) is,

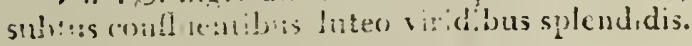

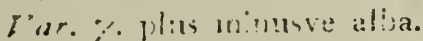

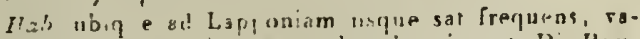
riefaies vern lautun in st I r calen ubvenierund. Di Par. hid!: at Vileso:zo Cum praceodante semina varia, ut ciban coligit. Didifican: a ts in remmbus ramulerum

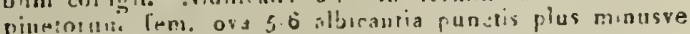

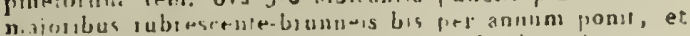

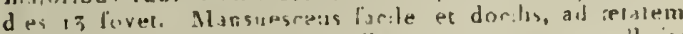

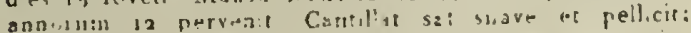

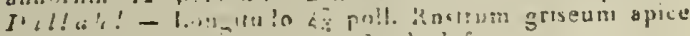

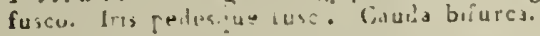

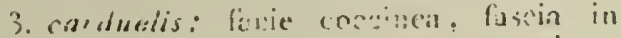

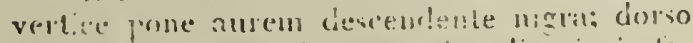

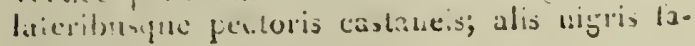
sitid laied. 

$f$ linea circa basin rostri rufescenle, albidine capitis laterale fuscente, tectricibusque alarun minoribus fuscis.

Linn. S. N. 318 . 7. Fn. 256 Ret3. Rn. 227. Nilss. Oin. 73. Fn. 351. 12. Hamns. Fu. 2i5. Fringilla, - Sv. Zool.

So. 'l istelfröfink, Stiglitz. - N. Steig. litz, Stilliz.

Hab. ubique, sed non m!gnis. Victus pracedantiam assumit. Nidulatur inter rainislos arborum, ubi fem. ova 4-6 oblusa glaucescentia maculis strichs:ile ralesciavibus ponit. Pullus femineus post exclusionen fiscinla rostri alba cognitus est. Viridus et acrilis centullat son:t svari et tinnieule, facillime mansuescens $M$ ad anmos 24 osque virens Pellicit: Ziflicstichlick! In cantu suo nuelodo comnodulaliones cum fink exprimit. Longitado $5 \frac{1}{\frac{1}{2}}$ poll. Iris castanea. Hecies paliide-fusci; cetero characterbus indicatis sat rognitus.

4. linerin: ") rostro flavo, stria supra subtusque longriludinali nigra; vertice enccineo; gula nigra; alis caudayue fiscis, fasciis 2 albidis; pectore m. (prseospus iempore vernoli) sanguitieo.

$f$. pectore hypochondriisque evidentius nigrofusco-mantulatis, illo liand saicruisito.

Linn. S. N. 322. 29. F11. 241, Metz. Fit. 237. Nilss. Orn.

-) Duobus exemplaribus operis Rurberkii, avium piccarum, at Surcea prossontibus a noless examind.

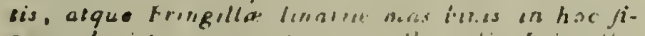

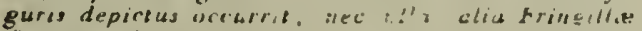

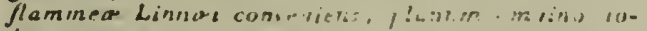

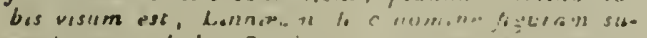

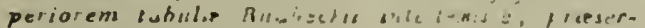

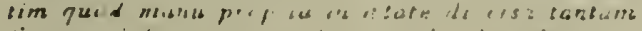

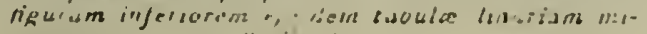
nurein iomenusis ilidieck.

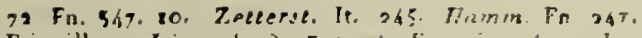

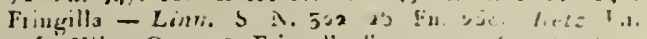
225. Nilss Oru 75. Fringr!la lizumes. iv /...!

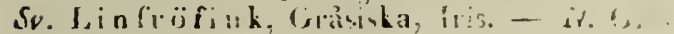
sidske. Nionrrisk?

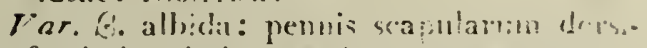

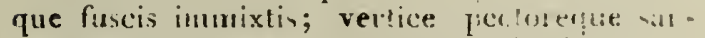
guinescentibus; alis caudarue fiscis postiss albis sparsis.

Vor. $\gamma$. alloida: maculia langi?udinallins supra numerous sululus ravioribus fuscls: iei. tice pallide coconneo.

Hal. sxpe at prosertion hime gregatim in 1010

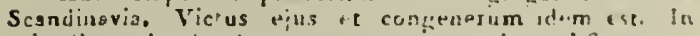

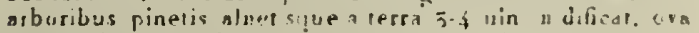

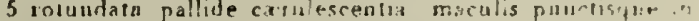

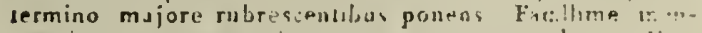

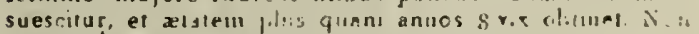

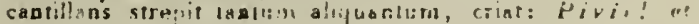
pellicut: kreck kreek hvit! - I.nngitudn s: poll. li.

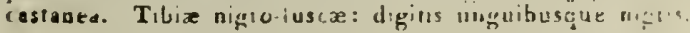

G. 16. CANNABIA "), Sr. Himpling.

1. propria: castallea: "ablice cisereo irn.

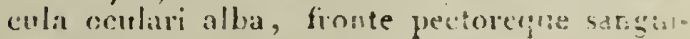

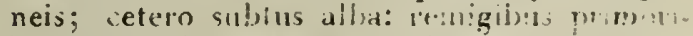

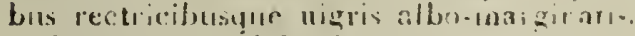

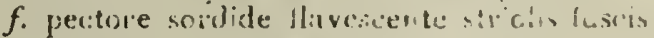
Linn. S. N. 322 28. Fn 240. Retz. fo 220 Niles. Uru 70. Fn. 340. 8. Fringlla canilibilla. S2. Zool.

-) Hab. genes, percedertis. Ninmen gener e $i$.

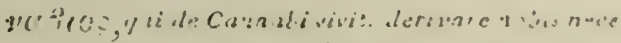

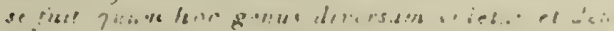

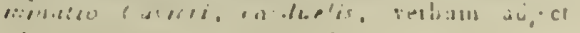

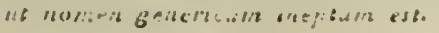




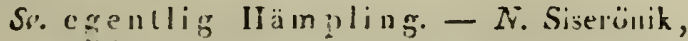
Silite.

Hab, in Scandinaria media el ansirali sal frequens, xslale in IIntirelis, hrme all arris. Semins el plante variä victur ejus; si mallshota e selmuibus cannabidus iantilim a'ilur, miuns prosuras. In filificibus nidulalus ora 5.6 ovatia cieruidescente-alba jugctis in teimino majore iucarnalis nonit. Cantilat prorenge ad rantus disersus etjam docilis, - Iangiludo $5 \frac{1}{8}-0$ proll. Linitium sunra cxruleu-atrum, subtus \%risen-lucirnatum atice nigricante.

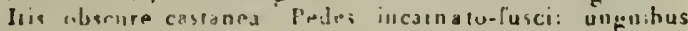

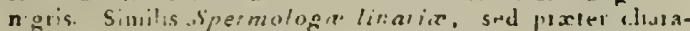
cieres genericos et surciticos indicatos masumtudine minore et collore dossil it eo d.firer.

2. Jlosirus:-ls: *) asigricans: roclro flavi. cante, initrginibus permarmu, gula pectore-

-) Ob forman roetri merito ad hor genus pertinet,

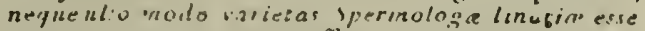
potest, a quo ansuner ditiert corpure majori, ro-

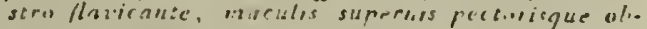

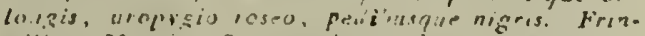

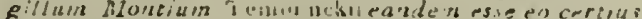

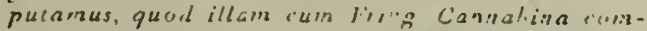

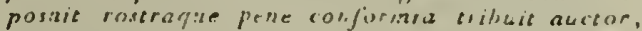
et feminsin culem. qualem sista finira liuchberhii.

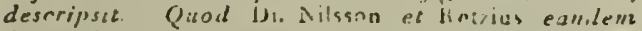

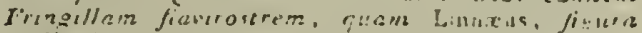

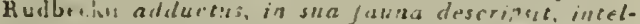

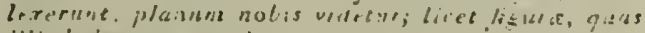

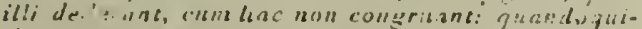

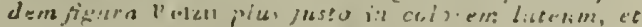

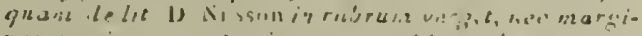

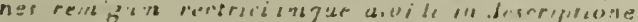

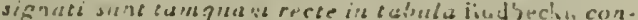

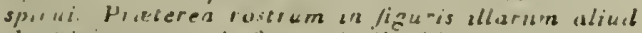

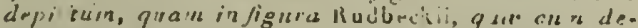
sriptione fringiblu montium a D. Ceutuniack cis?. magis convenic. que nigro-maculato pallide rule aentibus, al). domine: allsido, mopivgir, lesen.

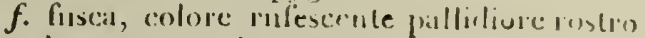
que lutencente apoire nigru.

Linn. S. N. 322, 27. F11, 20jo. Nilss. Orn. 71, 1. 4, Fin, 3.4. 9. Fringilla - Retz. Fn 22-. 1. L. I. Finnulla liniria var. Mudb. Ar. 1. 55. Frungilla finsca rostro flivicaute ( $f$ ?) Leem Beshivelse orer Finnuakens Lupper. rag. 256 . Sin. Zool.

S2. Crulnafibllambling, liiskil. $-N$ Giali lirisk.

Hab imprumis in Nimprlaulia Suacira et in Finnmur. chis Sudfirlidapue Norveg.r raissuma of vix alibu ve! migralls. - Longitulo $5 \frac{1}{4}$ finll. liosirum indis autumno biemeque pliam apice nigho, coles rufescens tunc pall, diur, lese ut in lemina et jun,oribus: el urnpygium mag's rulescente-1incium maculis nigris. Is is brunnea. Pedes n'gri.

G. 17. EMBERIZA : , Sv. Sparf.

•) ungrue postico parro, curvalo.

1. cierinella: supia rulescens milnulis nigris, sul,tus vitrina, peclure superinie fiseco. maculalo; capils citrino crevice olivacea.

$f$. cappite olivaceo, longitudimalier nigro. maculato.

Linn. S N 30.7. 5. Fn. 2jo, Retz. Frl. 219. Nilss. Oгн, 80. Fn 299. 2. Hamin. Fn. 24t. - Sv.Zoul.

Sv. GulSpalf, Gul-pink, Gı̈̈нing. - N. Gulspirrv, Shir.

for 3. Lota llavescente allia.

-) $\mathrm{H} 36$ gener. medius inter Fringillas el Alaudas. N. gea. vetustum Gesneri derivatione ignotum. 

Var. $\gamma$. tectricilmus pallide luteis.

Hab. ubique sat Irequens; varietates vero raciesimx. victiat estate insectis variis el larvis, hieme semin:bus cereslibus et aliarun plantarnm. Nidulatur in Iruticiluss ora sordide alba maculis striolisque nigrescentibins ot brunnescentibus bis per antiti ty yi! pellicit zip cubans. Cantillal ti i ia ziap zöre. Cara ejus $z a p$ ! et volitans criat $z i a p z i a p$ et sapidissima. - Longituilo circa 7 poll Roarrum supra nirricans, subtus flavicans. Iris fusca. Cauda bifun suche-carnci digitis el unguibus nigrofuscis.

2. miliaria: supria Junnescente-grisea subtus albicans maculis longiludiuslitus nigricantibus; cauda nigı-fusca albido-marginala.

Linn. S. N. 308. 3. Retz. Fu. 285. Nilss. Orn. 78. Fu. 234. 1. - Linn. Fn. 228, Hamm. In. 240. E. calandra. Sv. Zool.

Sv. Hirs prarf, Kornlärka. - N. Kıfolter, Kornlerke.

Hab. in provinciis australinribus Srecire Norvegipque in arvisque, non infreicuens, migrans. Nomen vu!pare Kornlärka, propeer simitiudinem alaudxe quoad coforem, ei almbutum. Cantillat non insuave, sixpissimm ioi coitoitiritz, pelicil Tirjitz, el evolitaus ro-

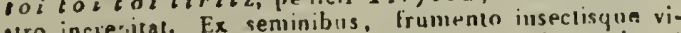
stro incie:ilal. Ed semisub firmicibus vel in arvis vel clum y'inerit. Nidificans sub truticibus vel in allide.grises pratis, muime in ipsa lerra, ovi 4 e niglescentibus pomaculis striolisque brunnescentibis ee nigiescentibus ponit. - Longitudo $7 \frac{1}{2}$ poll. Ilostrum suprs nigrn.fiacum, subtus luscum, inbercala pilati exserto. Lutera copitis lulescante-alba striis rufo-fuscis. Iris caslalled. fiul inumsculala. Siria juguli pectoriscpue subiriquetra. liemiges nigro-lusci: matginibus bunnescentegriseis; introstges nigho maruinati. Rectrices npice albicantros, extimne tecula obsoleta cuneiformi albida. Pecles pallide griss. thacule-lules: articulis digitorim fuscescentibus.

3. schoenichts: supua nigra marginibus pennarum mopygioquc rufescentibus; subitus alla, maculis hicunu capilis colligue pecto. risque fuscis, jurnli nigris.

$f$. supra dufescens miculis nigris: collo cinerco-tincto; lateribus capjitis rofescerititals fascia superciliari albicantc; collo sordide alloo, stria utringue decurrente nigio-fusca.

Linn. S. N. 31 r. 17. Fn. 23r. Retz. Fn. 218. Nilss. Orn, 8r. Fn. 305 4. Zetterst. Res. 1. 106. 2. 67. - Siv. Zool.

Sr. SärS parf.

Hab. in provinciis liyperboreis inter arundines sat fre. quens sed autumno migrans. In ipsa terra sub linticibus nidificat, ova 4.5 pallide-grises, nidculis striolss lumecti: curvis obscure-brunneistincta ponens. Cnumllat $i$ i $i$ iy tis! reitsch! et pel icit iss iss! Semina plantarum variarnm pracsetim litorsarum cibun ei prabent. - Longitudo $\delta$ poll. Rostrum nigicans sublus flavescens: tuberculo palatini parunt conspicano. li is fusca. Canda bifurca. Pedes fuscescenle-rubris ungubus nigro-fuscis.

4. rusticn: ferrurinescons: maculis dorsalibus nirreis, fascia supereiliari daplicirne in alis albis; subtus alha: maculis pecteralie bus inque hypochomblies fermerinescenilsus remigibus 4 cxterioribus xspulibus.

f. capile nigricanic: fuscia superciliari fer. jugineo-grisescente.

Zetterst. Res. s. то7. t. I. In 2. f. F. 'nnreslis. Nilss. Orn. 8t. E. Sclininiclus var. IF. 3cs. 5. - Sv. Zoob S2\%. La $11 \mathrm{~d} t S$ par $\mathrm{f}$.

l'csb. in Lenponia D:i Zercerscedt s: E. Fries, rao rius in Sconia D. Nitsson. Anne fixe svis cannla est, cujus canjus b excelientiss. Yirn Connite Skjoldrlirand (Voynge pilloresque au Cap Nord. rom. qo.) iz unitisu-

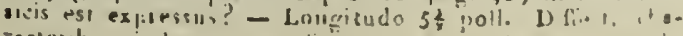

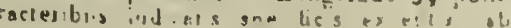


remige primo longitudinu 3 saquentium at ab E lappo. nica prefirea uninue posicico bresinie. Fiollitm filsolun subtus flavesens, in mare mogis tlavicans. Iris linse. Pedes lalescentos. Cauda nimo-linsia: sectrice extima dimidiation alıa sed apice exieriure lusco, 2. 2. macula cuneiluımi alba.

5. Lortulnna: capile colloque olivaceo-

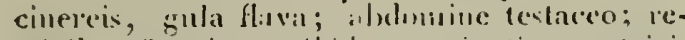

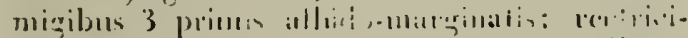

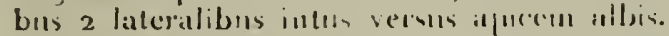

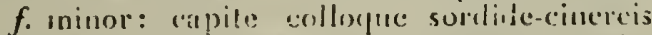
limeolis nighricalilibus pectore pallidione, sulaque luico-altu, (in statu juniore: a!lu,t).

Linr. $S$ N. Fug 4. Fn. 224. Rets. Fr. 216 . Nilss. Orn. .9. Fu. 302. 3. liull. Iv. ed. De Geer. 1. 76 jum. Sparrm. Nus. 1. al jun. - Sv. Zool.

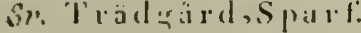

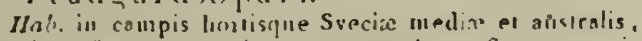

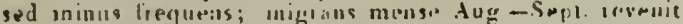

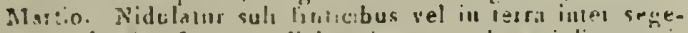

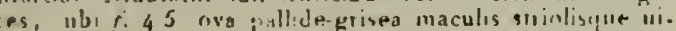
ris punit. Semira v.rir el illsecla cibus aids l'ollicie isvic, tsvel! rriat evolituns peak pech! p! dean. Wulans gye gye! Cantil:at sedens in raun'a bink, bink, iink, tink, tjohrr! - Lomullidu 6! pol!. liosirum incarnalum, subius rem pallidius: luberculo polati mimes conspicuo; narbus nudis. lic lusca. Pal.

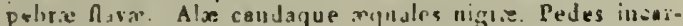
'atii, unguilous precipue postic', pallinlis.

-) nngize postico recliose, dipito longiotn.

6. nivalis: allsa: darso, a!is rambargue

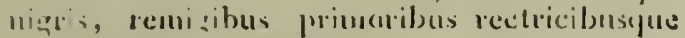
is a dalitus allis exceptis.

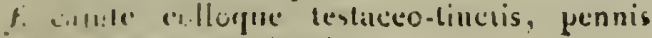
ii. al kobeco malginalis.

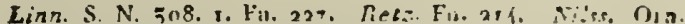

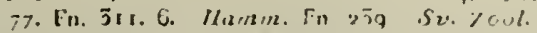

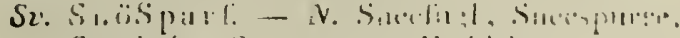

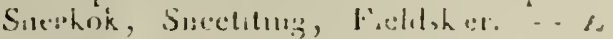
Aliıil:-

7 es 3. fasciar pecelmali amanticro.

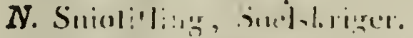

Hab. in Lappona. ated haesue instante ad prosiucias

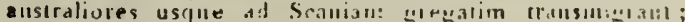

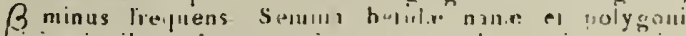

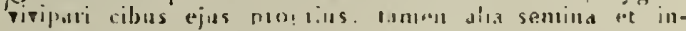

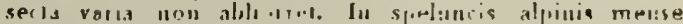

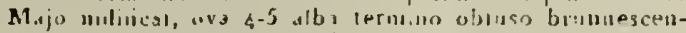
te uiculura non-us. Cautultal sal suave el pel!icit soulu

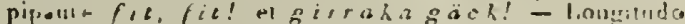

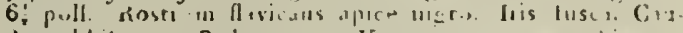

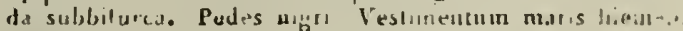

fese teinuma

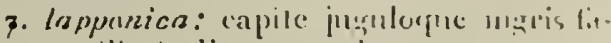

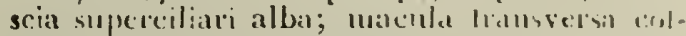

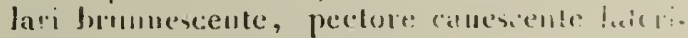

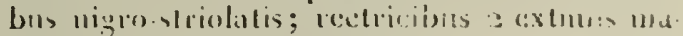
cular apical, cuncitommi.

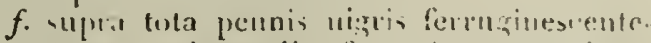

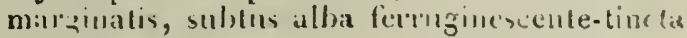
n.jpicaule-sluiciatlis.

Lunn i. N 717. 1. Fn, 275. Liets. Fu, arg. Limgilla, Nilss. O111 76. Fn, 3̈4. 7. S2, Zool. 2.

So. LappSipat.

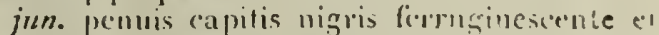

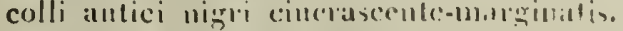

IIab. in regroubus alpinis I.Apponicis aure tell. ए.,

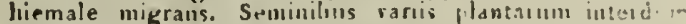
elidin insectis viclilat; in arsis ad tuminlos limmulos? ficat el ora 5.6 oblonge sordide lutescipnta misill is

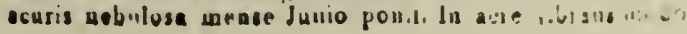



Alaudarnm estregie camtillar el zinzilulal fict! - Lon. gitudo 6 pall. linstrum luteuin apice nigro. Iris bruanescens. Cauda bifurea. Pedes nigricantes. Similis Pyre fitce domesticie, a qua tamen forma rostri characleribusugue indicalis valde distinclus.

\section{G. 18. PARUS *), Sv. Mes.}

1. mujor: capile colloque antice nigris, temporibus allas; dorso olivaccu-viridi; pe. ciore abdunineque flavis stria longitudinali nisgria.

f. minor: colóre dorৎali minus viridi striaque lougitudimali nigta breviuri.

Linn. S. N. $34 \%$. 3. Fn. 265. Retz. Fn. 255. Nilss. Orn. 122. Fn. 279. I. Hainin. Fn. 257. - Sv. Zool.

Sv. Stor Mes, 'Talgoxe. - N. Kiödmeise, Mlısvit, 'L'ulgoxe.

Var. B. supta vitescens sulotus flavis: maculis pectoralibus brumuesceutibu; abdöınine obscurcecurulco; rectricibns 2 unediis vire. scontilum, ceteris obscurioribus.

Strüem Sündm R. 240.

IJab. ubique frequens, restale in pinelis, hieme sd donios; var. $\beta$ rarissine in provincia Soenduloerense, D. Struem. Insectn varia, larvze, semina planlaium, bacce, pinguia quoque cadapera cibum ei prabent, ales alıs interdum interticit cerebrum illarum edens. Ad si) anuoe usque vivit. Ord 8-12 lutescente-alba maculis striisfue rufesceulibus, fen. ponit, qux cuin male alternatur fovet. Cautillat mon insuave: sitzickda, sitrickda-sichdi, sichdi; slidel: oizerh el

-) Hub. gener. fere pracedentium sed capite proporsionaliter majore et collo brevinre, alis longitudine corporis, remigilus 1. 5 longissimis; pedilingque validis, ungue postico validiore of curva. bivere - Nom geuer. labinum. pellicil: fink, fink. - Lonfriludo 5 poll. Rosırum nigrum. Iris fusca. Pudes plumbei.

2. acer: cajule colloque antice uigris, Jateribus albis, steia occipitis alba; dorso cimeren-cxrulco; sulitus sordide-alba.

$f$. rapite gularue nigris lateribus tantum (a) pitis allois.

Linn. S. N. 341.7 . Fn. 268. Retz. Fn. 25\%. Nilss. Orn. 127. Fn, 281. 2. Rudb. Av 113. m. Pulmstruch A. ves piclic 5. $m-S \nu$. Zool.

So. Svat 1 Mes.

llal, cum priore, sed minus frequens, eodemqua cibo gaudet. Nidificans in tera et cavernis nutorum rel in caris a, borum fem. ova 6.8 alba punclis badiis pnnens. Clanitat partan melodice zif: rifizifizifi! et zidadidadidadi! - Losigitudo $4^{\frac{6}{5}}$ poll. Rosirum nigrum marginibus mandibulatum pallidis. Iris fusco. redes plumbei.

it polustris: capile supra grulaque nigris lateriluls allois: dol'so caudaque betuluescentegriseis; smbills sojdide alloa.

f. uncucula gulaui paura.

Liun. $S$ N. 341, 8. Fn. 2tig. Retz. Fn. 25s. Nilss. Ora. 128. Fin. 286. 5. Palmstr. Ar. 60. m. Gr. f. Hamm. Fu. 260. - Sv. Zool.

Su. 'Tr. sk M c:s, Eilita, Telc, IIaupmeis.N. Giaa Mcisc.

Ihal ubique precipue in sylvis frondosis, juniperetis, horlis \&.c.ysat lirequens. Insectis, baccis Subi seminibusque valiarum plantarum vescilur. Nidulatur in cavis aborum, ova 8-12 ovalia argentea allo a maculis coccineis ponens bis ner aunum. Clamul clara roce dia dia! hitzi ïh $\ddot{a} h$ ! - Longiludo $4 \frac{\mathbb{A}}{4}$ poll. Rostrum nigrum. Iris castanea. Ale caudaque nifro-fusca, leniggibus rectricibusque extus albido inarginstis. Pedes plumbei.

4. sibiricus: supral rubrescente griscils, subtus albus: capite antice brunucscente; gula 
juguloque nigris lateribus capitis collique albis abdomine rubro tiucto:

Linn. S. N. ed. Gnel. I. 2. 1013. 24. Nilss Fn. 284. 4. - Zetserst. Res. I. 240, 250. 1. 3. Parus lugubris. Sv. Zool.

Sv. SiberieMles.

Hab. in sylvis Lepronize ad Jukkasjärvi sat rarn; $D$. Zetterstedt. Nulrimenium et mores cum congeneribus earlem, sed cantu suo P. atro approximat. In cavis Pini sylvestris circa 2 uln. a terra nillificat, ova 6 ponens. -

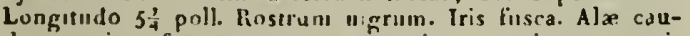
daque nigro-fuscr, meroune exteriore renigun reciricumque pallidiore. Pedes plumbei: unfuse postico valido. Prater charactures indicalos a 2 proxime antecedenribus rostro caudaque proportionaliter longioribus ungueque postico validiore differt.

5. cristatus: rufescente-griscus, subtus alhus: capite allo, crista aculiuscurla pennis atris albo-marginatis, gula juguloque collarique nigr.is.

$f$. rrista capitis minne, nigredincque gulxe jugulique minus repandis.

Linn. S N. 540. 2. Fin. 266. Retz. Fn. 254. Nilss. Orn. 126. Fn. 283. 3. Hamm. Fn. 258. - Sv, Zool.

Sv. IO fistles, Tufitita, Toppmes, Tofsmös. sa, Mislialt. $-N$. Topinejse.

Hab. in pineris non infraquens, presertim scandioarix inedix anstralisque. Nidum suum in cavis arborum l. spe?uncis moncium preparans, fem. ova 8.10 alba maculis in exıremirate majore plus minusve couflaentibus sanguineis ponit. Insectis seminibusqua pini et annubidis viclitat. Criatsono strepente: krrrit, krrris! \& Gorrky. - Longiludo tt poll. Rosirum nigrum. Isis fusca. Remiges griseo-fusci extus rufescente-tincli. $\mathrm{Hectrices}$ griseo-luscee, lateralibus versus basin exius ru. fuscante-albis. Pedes plumbei.

i. oxtrulous: supra olivaceus, subtus fla. vus: capite alloo, vertice stria abdominalialis candaque caruleis, line'i per oculos gularue. nigris.

$f$. paulo inino uhique stria abelominali et rolore miturs pulchiro.

Linn S. N. 341. 5 Fu. 267. Tetr. Fn. 256. Nilis. Ors. r23. Fn. 288. 6 Hamm Fil. 259. - Sv. Zool.

Sv. Blâlles. - N. Blaa Meyst.

Hab. restale in sylvis, hieme ad domos sat frequens. Nidificat in cavis arborum, oya 8-50 rubrescente.alba. brunuescente-maculata. Sonum Pyritio doinestica si-

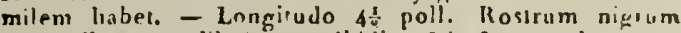
marcinibus mandib,larum alhidis. Iris fusca tlie erruleae, lascia, nargine exloriore remigum mrimorum apicibusque secundariorum albis, lasereque semigum interiose nigricante. Pedes plumberi

7. cyanus *): pallirle-cxrulerus subtus albuy: capite colloque albis, stria per ocrlos, fascia. lata nucluali, tectuicibus superinıbsus alarum, remigilus noumullis cauduytue supı a ubscure-crenteis.

Iinn S. N. ed. Ginel. I. I0n7. I6. Retz. Fn. 255. Nilss. Orn. 124 Fis. 289 7: - Lirn. S. N. $345.1 \%$ Г॥ 8 4. c. fig 1. 1. Lanius biarnicus (1). Sparrm. MIus. 26 . P. sarbyeosis. Sv. Zool.

Sv. Högblà Mes.

•) Lanium biarmicum Linnei eundem esse ac Lanium caudalum contendenti $D_{i j}$ Nilssun a.sencire num possumus; compalatis enim desrriptuster ef figura: cum macula nuchali transversali. colure alar:u:n caudeque ore, quas Laniu bi irinicu deilit Janue: ne dubium quidem fieri potest, quin Paruni cyn. num intellexerit. Quandu vero possea altern spe. cies, cui idem nomen, binrmicus, facen est, deno. minationcm Pallatio ed errorem evitandum, proti sulimus. 

11.23. in Susermannin rassima; D. sparrman. Vesciell itr-clis at semmbas siculi congrneres. - Langisorlo $5:$ poil. Lisstrum Inseun inargumbas mandihuisrulu slbud:s. I is finsca. Peclus permins 2,3 obscule-cacbateis madin instructuns. llamiges primoles fusci, basi exceppo murgine exterinre albi, secundarii apice tectricrasguro interinows albi Rectrices laterales extus dimidiala? oblinge allix. l'edes obscure-cervalei. Unde medius ridelinr inter Panm caruleum el caudatimn; sed priore nuajur corm calldis Inngiore.

S. coudutus: supua niget albo et josco varius, capite albo; s!lislus albus: alulouniue crissonut lufesentibus; cauda cuncilomi cor. pore longiore.

Lin!. S. N. 342. 11. Retz. Fn, 259. Nilss. Orn. 125 Fin. 290. 8. Kudb. Ar. 114: - Linn. Fn. 83. Lanius. Sv. $800 \%$.

Sv. Svaus Mes, Allila, Altyla.

IIab. in sylvis densis et irulicefis passim, hiame rero magris grty.tim in alnetis. Insectis rariis, lavis brisque eorum victirat, ad truncum arborum nid ficons, et ibi ova 12 ad y' usque alba punctis in exiremitale majuse lulis

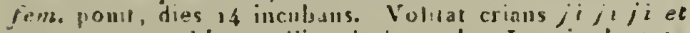
ge ge ge gels! et pellicil izirrre! - Longindo tola b, candia $j \frac{1}{2}$ poll Characteribus indicatis valde disersus; Knsirum parvil nn nigrum : basi pennis reversis oblecta. lis nigro-fussca. Siria nigricante superciliari a rosıro fomina dislincla. Cauda nigra. Pedes obscure-fusci.

\section{Nat. 7. ALAUD FIDES, G. 19. ALAUDA *), Sv Lä:kz.}

- Il abit. gener. ad Frillg lleidas apprnximatsed capile proportionaliter minure et collo longiore; alis medincribus renige 3 longissimo; ungue pedum postiro rectiusculo pollice longiore. - N. gener. labinum.
1. arransis: grisco-rufescens maculis fu. scis, subtus rufesceule-allą: pectore maculis minutis fiscis; caula bifiuna: recluce exlima extus supra medimn oblique secunda exlus apiceque alba.

f. paulo miuor: pectore pallidiore.

Linn. S, N. 28\% 1. Fn. 209. Fietz. Fn. 19\%. Nilss. Orn. 118 F11. 272. I. Itrmm. T11. 222. - Sv. Zool.

Sv. Âkerlärka, Săngläka. - N. Lerke, Sanglerte.

Yar. R. firuginosa inta vel nigia.

frar. $\%$ remigibus rectricibusque plesisque albidis.

$V a r . . \delta$. tola alba vel luiescente-alba. josn. colore magis variegato.

Hab. per Scaldinarian fere lolam, certissime hieme mit osi manens, jamdudum mense Jan ad Holmiall occisa; sibb nive hibentuat. Inserla, larve el semina varia victus ejus. Callus ejus vibras tirili-tirili.tirisirliri janjam o lempose Taubnauni cognitus est. Pellicit feminsm in amorem terle, alltumno sunat dries. Nidulatur in cespiribus agrorum vel camporum, ubi ova 5 albo-grisea punctis minculisque griseo-fuscis ponit. Longiluilo $m$. $7 \frac{1}{8}$ pull. $f 7$ poll. Riostrum fuscum subtue albidum apice fusco. Iris griseo-finsca. Gence sirioguo supra oculos lere collari snidide-albx. Remiges obscurofusci, axtini 5 requales margine exterione albi $5-14$ apice albi, excisi, margine extelince ferruginei, is lonuiludine quinti cum sequenthius margine pailide-urisei." Pedes fusco incornaci: ungubus luscis, postico recio apice albu

2. arborea *): pallide-lestacea, sublus

-) Avis sub nomine scanico Topplärka (A. crislalella Latli.) e Scania Lerit. ill. D:o Thunberg missa hie et non antecedens fuic. disubla criofoba lieiz:l b'to 
albicans nigro-maculata: stria albida caput cingente maculayue flexur:e alarun alba; cau. da bievi xquali.

$f$. mari similis sed albedine puriore.

Linn. S. N. 287 3. Fn. 21 x. Retz Fn. 195. Nilss. Orn. 119 Fn 175. 2. Hamm. Fil. 234. - Sv. Zool

Sv. 'TrädLäıka, Skngslarka, 'lopplärka, Titt. - N. Skovlerke.

Hab. in pinetis provinciarum anstratium. Viclıs ejus iden ac prioris. Ad finem Sept. emigrat, rediens Mlarric. Sub fruticibus junipers et in ericetis nidifical, ura $4-5$ alurale-grisea maculis fuscis ponens. Claniat volans: Ledigoi! - Differl a nracedente prater chalacteies indicatos Inngitudine lantum 6 poll. slatura breviure sed fere crassiore, rosiro tenuiore, colore supra nbscitriure maculis ninnribus, sublus maculis cuneiformibus in juculu moribus, lateribus colli nuchaque albidis: moculis nigricantibus; pedibus pallidiuribus: ungue postico palJido, compresso, recto, apice sub-incurvalu.

Coh. 2. Esangmatroostnes: rostro ad apicein emarginalo:

\section{Nat. 8. MOTACILLAIDES,}

G. 20. ANTHUS *), Sv. Blomärla.

-) sostro ranuiore; ungue postico longiore.

1. praconsis: olivaceus maculis uigrican. tibus, subtus albidus: tectricibus alurum majoribus apice albidis; rectricibus nigricantibus extimat extus dimidiato oblique secundaque macula cuneiformi alba.

Su. 198 nullo modo Scandinrtvire, teste $D$. Nilsson in Orn. Su. p. 257. "Isa fuit.

- Hab. gener fere precedentis, sed remigibus 3. 4 longisssmis; unguse peduns postico plus minusve curvato. - N. gran. e greces voce: uvVOs, flos; (Nomen svic apud Aristotwlem). f. nisurofueca uhi m. nigricans: marghinilus fennalum olivaceis.

Linn. \$. N. 2\$7. a In. 2ro. Rete. Fn 194 Kamns Fn. 223. Alailddo - Nilss. O11 114. Fil 260. 2. - S21. Zool.

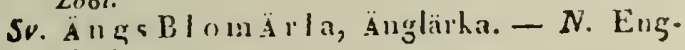
Irike.

Hab. per lolam Scandinaviam in pralis humidis; mi-

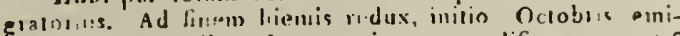
prat. Sub fruricilus Juuper in terra alifical, ova $4-6$

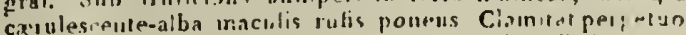
bes lis lits l. ist ist - ist ist ist! pellicit tien tick tack! Pt cantillat usave voce vibrante. Insrctis Jarvisple vititat. - Lungimblo 6 roll, inter Antilus swandinavise igitur minimus Rostrum suprs niglieass, subtus rufirluteum apice fusco. Tougua lacerd lis huun-

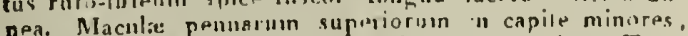

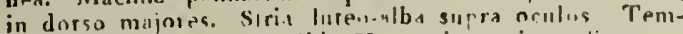
por? fuscesienti.. line : albis Urunyuium guisen-olivacisuna

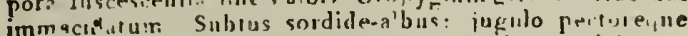
sulphuesicale linctis maculis nigricanibus. Ablomen et rriscum sordids-atha. Remiges niguicantes, quntum prinus magine prosiore raniter albus, cetai maruine viridelaten in intim: 3 l.trote, tecnicibus nigh camr bus: minuribus li-nniter olvacoo-, majoribnaque apice susdid. albo-mirgiuatıs (unde strixe bine in ala complirala). inferioribus luscescentibus ltiten-timetis, Canda snb-bifuca: rectribibus, extimis axceptis, margene exterinte talliter luteo-rirescentibus. Pedes pallilie.incarnati: digitis lucescentibus, unguibus luscis, quorum posticus parum curvatils.

2. uhscurus *): fuscescens maculis obso.

-) Quia D. Nilsson maxima fide ostendit hane Lin. naro ignotam vel ut propriain indeterminatum specien esse Aloudain obicurain Lallami; caursiam nullom, quin hujus dennininationem haud inepran, ton retineamus, habusimus. Qualldoqui',..n hace species, eadem sille dubio est, quam Spias- 

letis obscurioribus, subtus sordide albus ma. culis fuscescentibus: rostro uigro; rectrice extimui extus obliyue sordide alba.

Linn. S. N. ed. Ginel. 794.4. Alauda cainpesiris B. Nilss. Orn. 115. 1. 9 f. 1. senior, 2.junior Fn. 257. 1. A. rupestris. Sv. Zool.

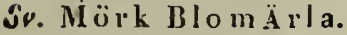

junior: subtus llavicans remigibus nigricantibus, primoribus margine exleriore tenuiter lutco-albis, secundariis extus latius olivaceomarginatis apicibusque tectricun majorum albidis.

Hab. in rupibus et saxósis Scandinavize australis et occidentalis, haud vero frequens, ad Helgolandiam et Salten Norvegix usque, ubi nidifical inter gramina, ova 5 sordide-alba cum naculis luscis in extremilate obtusiore obscurioribus el fere confluentibus ponens Clamat $h i s c h$. hisch! - Affinis pracedenti et medius inter hunc et sequentem, cujus lanıum varietateın D. Gmelin credidit; differt tamen b hoc primo intuisu ungue postico longiore el ab illo longitudine 67 poll; rostro nigro basi 1. subtus vix paltido, stria supra at pone oculos albida (in Ggura U. Nilssoni tamen exclusa), colore tolo obscurinre, rectrice extima latere exteriore et oblique per apirem sordide-alba, secundaque apice pallida, rectricibus nterntediis cinerascentibus et pedibus nigro-fuscis: unguibus nigris; postico mudice curvalo.

-) rostru rubustiore el ungua posticn breriore.

- 3. campesiris *): griseo-fuscus, subtus

Jeltam denominavit $R$ ajus, retinendum quidem hoc esset nomen, de latinn vero non desumium lingua, rejiciendum est

-) Jam dill Alanda campestris fuil ab auccoribus inanca et non absoluta descriptione, quan immort. a Linnè dedit, cominutata; quum vero alia specics Alaudae diligenter ab eo sunt deberminabre nlbicans: striis in pectore fuscis, superciliis lierugrinesentibns.

f. subtus sordide-alba: tinctura pectoris ferrugincscente-lutea levi pertoreque fere immasulato striis variis firsco-griseis.

Linn. S. N. 28S. 4. Fu, 212. Retz. Fu. 196. Alauda. Nilss. Orn. 16. Fn. 267.4. - Sv. Zool.

Sv. Fült]3lom $\ddot{\mathrm{A}} \mathrm{l}$ I a.

Junior: magis variegatus obscure-fuscus pemuis lestaceo-marginatis, subtus sordide-al. fus: liseo!a ad latera gula utrinque nigra peclore luteo-tincto, nigro-luaculato.

Hab. in campis arenosis Suecixe australis minus frequens, migratorius. Nun canti!lat, sed altissime volitans clarnilat zivoly, zizoly! l. zirhy dasida! pellicil in terra $d j \ddot{a} c k$ djäck! el territus sund zirrp! Nidulatur in terra ova 6 a!ba fusco-maculata poisens. Magnimdine lere pracedentis I. longiudiue prope 7 poll.; sed iostro magis elongaso, supra fusco, subias albidu, apice lusco. Lingua apice bifids. Iris fusca. Color corporis supra aliquantum in olivaceum vergens: Inacnlis longitndinaibus rachideis. Lropyuim tectrices que calla? superioses, quarum binic ultra nedium caude elongatir, acuıninatr, immaculatie. Lines a naribus sifira oculos rersus nucliam ferruginescente-alba; linea aliara a sinu oris sub oculis ad latera gulie niguticans. Lora fuses. Teinpora fuscescentia. Sublus sordidealba tinctura prosertim in pectore ferrugineo-lutea: laterbus inguli poclorisque maculis parvis rasioribus longiludinalibus fuscis.

ef descriptio ejus anarima parte cum hoc congru. ere vid-bur, synourmon ejus heic rebiucre dubiusn est nulluin. Sentention D:i Nilsson Aloudain campestrean l. eandein esse ac feminam Saxicolie 14 betre sequi uon possumus, quia sogacistimum Linneum, qui sam uritis Alandx ef Notacillae geenera limitibus disjuntut, hauc ignovisse. haud ire. dere fus est. Churucier, quen Al. compestri l. C. 
Remiges fusci, primores lenuiter, secundariilatius margine exteriore [errupulescente-alliidi, ut et lectrices, inlerioros vero albida litieu-linsia. Cauda subaqualis fusca: reclii.e prima fere a basi extus dimidiato oblique-alba, 2 cilngren-fusca, magis acumiualx. l'edes pallide incarmali; ungue postico fere rectu.

4. srivialis *): grisen-olivaceus, fuscomaculatus, subtus albus: ugulo pectorecute ferruginescente-Inteo tinctis, oblon go fusco. maculatis; rectribus fuscis: extima extus dimidiato secundarne apice alba.

$f$. colore pallidinie.

Linn. S N. 28s. 5. Retz Fin. 197. Alauda. - Nilss. Orn. II7. Fn. 264, 5. A. arborrus. Sv Znol.

Si. valulig Blom Ärla, Piplärka.

Junior: Antho pratensi juniori similis, sed rostro breviore et robutiore, maculis pectoris majoribus, rectric: bus apice acuminutis et digitu poslico brevinre et curvato.

Hab. ubique sat frequens. Migrans in mense Sept. reventit medio Maji. Nidificat ill terr.s et ova 5 grisea maculis rufo-fuscis bis per aunum? pout. Callum sulum suavelt mas roce $z$ ia $z i a z i a$ ! cuncludil; pellicit tem. pore.

tribuit: corpus supra maculis nigris mora scolo. pace u in - - - tectrices aliruir nigro-fuscz. -l bo-ma rginat anguis pusticus iulermedin pauto loilgior, ut hanc speciem potius guam Mlutacillie ruberre feminam, si non juniorem follusse pra se habuerit, suasit. Nrieberca "lulli hujus generisspecioi Scandinavia hoc noinen bibui potest.

-) Ne duas species sub eodem nomine Jingant, quibus nostra distinctio inter Allthum el Alaudun hesud arriserit, hanc denominationem retinendain esse eo magis judicavimup, quod Lilltaxus nullure nisi hanc speciem pros ie habuisse videtur. pore coẹundi zip zip! ceteris temporibus $p$ is $p i$ piuspius! - Longlludo 6 pall. Rosirute hirvs, supra obscure-fuscim, sublils pallide-inctinnlia:n: ople $\mathrm{fu}$ aco Iris fusca. Maculie corporis lonkitudinair, ill capire minrres, in dosso majorres, stria a nalibus si:pra oculos albicains at tempora griseo-fusca. Mirulie in la. tesibus juguli nikro-lusce strian uirininge desrendentem

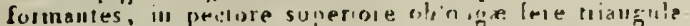
res, in hypochesndais linearas. $A b: \ln : 1 m n+1$ rissum al.

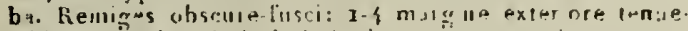

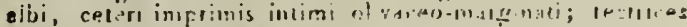

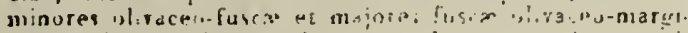

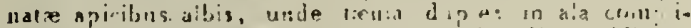

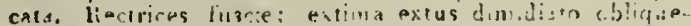

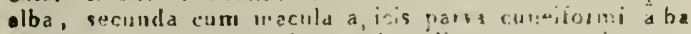

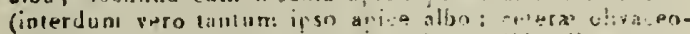

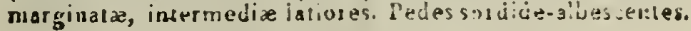

G. 2r. MOTACilla ${ }^{*}$ ), Sv. Airla.

-) ungle postico mediocri:

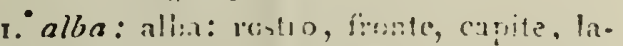

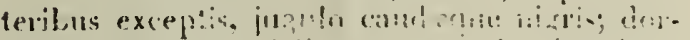

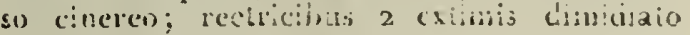
oblique-alitis.

f. srimble alba: nigoculneque cajilis cince reo-inlermixis.

Linn. S. X 5ir. 18. Fn. 052. lie:s. Tn. 2 7z. Yilss Orit. 112. Fn 250. I. IIumm. Fis. 251, - 22. Zus!

-) IInb. cerier. fure Antivi, sed corpore minlis robli-

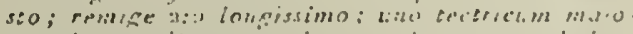

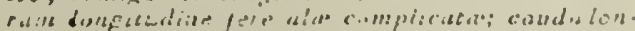

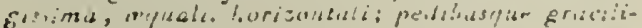

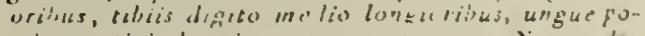

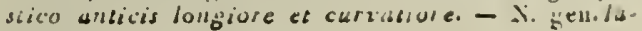
rinum. 

Sv. Hvitärla, Sädesärla, Kokärla, Riug. ärla. - N. Erle, Linerle, MariEile, Quekstjert, Elvekonge.

Junior: sordide-einerea, capite obseuriore, subtus alba: fascia oculati et fascia in itroque latere juguli descendente lunulam tians. versam formante nigricantibus; latcribus capitis eollique fusco alboque imunixtis.

Nilss. 1. c.

VIab. ubique, soepiosime solitaria, ad ripas, in plaleis, reis, pascuis pratisijue currens, caudau celerrime

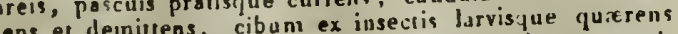
et captans. Currens et sursunz volitans clamat $g u i$, et captans. Currens et sursun volitansivit - Nidi$g u i, g u r r i r i$; sedens pelicit 6 Geat in catervis lapidum et truncorum, rel in cavis arborum et murorum, $\alpha c_{1}$; ova $5-6$ carulescente-alba macolis nigricantibus ponit. Migrat autumno serotino et revertitur ineunte mense Aprili. - Lollgitudo $7 \frac{1}{9}$ poll. Iris fusca. Dorsum, scapu!x, lectrices alirum mineres, lafusca. Dorsum, scapulx, ectrices abdominis crerulescente-citlerea. Remargine exteriore cinerascentes, interiore ad basin albi, latius vero in remigibus $17-18$, interiore ad basin albi, latius vero in remignibus a tectricibus longioribus utriusque ordinis nigrescent.bus, ap ce et margine exteriore albis, unde fascix a albx in ala complicats. Rectrices intermedix

-) ungue poslico longiore (Budyles Cuv.):

2. Thunbergi $\left.{ }^{\star}\right)$ : olivacea, snblus flava:

") Ob caput nigrum hanc avem, ultra Lapponiam non visam. ut varietatem Motacilla flava, licet habitus quodammodo similet, habere nequimus; idooque Viru de ornithologia meritissimo, discipulo ot successore felicissimo Linnet nunr deplorato, inter aves sisecanas gratum nomen dare voluimus. rapite nigso, gula alba punctis nigris; jugulo iufurote maculis fiecis (a)uflueutibus.

Nilss Orn. $1 \mathbf{3}$ M. Alara var. $\propto$

Sv. 'Th hu nbergs Ärla.

Hab. in Lapponia ad Fnontehis el Enara rarissima D Thunberg. Mot. Ravie aliguantum sinilis; sed characteribus editis ab ea valde distincta.

3. bosrula *): cincrea subtus flava: linea alba supra infraque oculun; gula nigra; reetrice prima totis, secnuda latere interiore ct. apice, tertia disco alba.

$f$. et $m$. autumno absque gunla nigra sed rubrescente albar lineis or ularibias matsis flat. vescentibus, colore superno olivaceo-tit:cio sub. tusque pallirlins fiavo.

Linn. Mantissa plantarum p. 527.

Sv. Bosk a psïrla.

Hab. in pascuis ad Fahlunam Dalecálise lempore sementis, boves iestate gregarim tota dite sioguens, Stomoxydes (Conopes) irrilantes ef calmurantes colligens. Insectis rariis vescitur. Nidulatur inter lapides, ad $t$ aculiuscula, ad exllemitalem magnam value dihtato.fl. ba maculis rubrescentibus ponit. "Colamat: tsibsitsei. - Longitudo $7 \frac{x}{4}$ poll. Rostrum nigrum liis fiesca. 4. flary: olivacea sublus fluva: leciricis bus 2 lateralibus plus dimidio oblintic albis

-) Hanc avem, in reliqua Europa natissimam, spe. ciem esse disfinctum, et turdem cim Mlotac:la boarula Linn. cestante jigura ab allo cstata, dubium non ess, et quum eain Lalecarlice observaris Linne, ubi diu inoralns est, Fallna suecica nec excludere, nec cum Mosacilla flava conponer fas est De gula nigra, qux in fein. semper ef in mere post plumarum mutationsm, des:. in descriptione nullum fecit inomentum Linne, ? yic constantem non inventerit. 

52

f. cineren.olivaceâ: gula jugnloque sordide flaveicente-albis, abdomine crissoque flavis.

Liun. S. N. 53x. 12. Fu Sv. 115. Retz. Fn. Sr.240. Nilss. Orn. 115. Pn. 253.2. - Sv. Zool.

Sv. Gulärla.

Junior: capite dorsoque olivaceofuscis, stria supra oculos flavescente-alba, supra quam fascia nigricaute, percue oculos fascia dilute-fusca; subtus sordide lutescente-alba: stria ad Isf(i)a juguli ntriuque nigra descendente ad inacstanu pectoralem.

Yar. 3 . olivacescente griseo-fusca: stria a nuribus ad superiorem oculi marsinem alba, alis ubscure-fuscis fascia duplici obliqua alba; sublins alba: jugulo anteriore pectore crisso. que laicscenlibus.

Lion. \$. N. ed. Gncl. I. p. 962. M. Tickurscbensis. Nilss.

1. c. var. (íe t. 8 .

Fnr. $\gamma$. tola alba, capile luteo-tincta.

Sparrn. Mus. Carls. t. 74 . Muscicapa albs.

Hals. prosertion in pascuis connes pecudum frequenies, varr. $\beta$ et $\gamma$ tamill ad llcluisin, D. Balleng, rarissine. Mligraus merse sept. revenil ad finten inensis A pilis. Nirlificar in pralis et inter seples, ubi ova 5.5

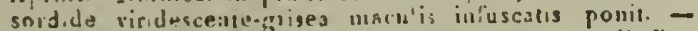
Clara race sonat sapl, sipp! - loagitudo 7 poll. Kisstrum nigricalls basi sublus polliding. I1.s fils:a Capul

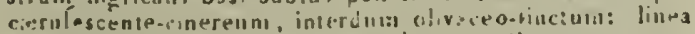
- naribus supia oculus versus niclisin a'ba, siriague a

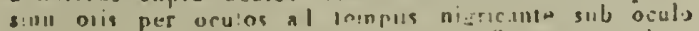
albo-iuterripla. Crupveiun dorso naghis flavescens, gul, et crissum flavescente-allan, celrorosubius flava maculis obscuris (rarn nullis), in jugulo ismon paucrosilou. Ale cons.

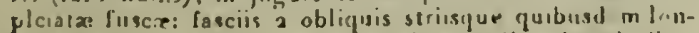
giludinalibus, albu-llavescentibus i teciracibus iaterioribus
Ruscescentibus Aaro-tincris. Reclrices extima basi et ad marginem interioren oblique nigro-fuscre, tertis unarg.ne

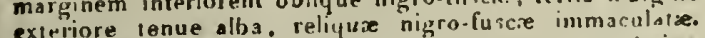
Pedes nigricantes. - Mluscicapa alba Sparrnanibi sine dubio huc pertinet.

\section{G. 22. $\triangle$ CCENTOR $\%$, Sv. Tonärla.}

1. modularis: rulo-brunneus, nigricante. maculalus: eapile, colio pectoreque sordide cærulescente-cinereis striis firscis, supta capite collocine.

Linn. S. N. 329. 3. En. 245. Retz. Fu. 233. Motacilla.Nilis. Orn. 104. Fn 1,3 x. Sylvis. - Rudb. Ar. 60. Rudbechisna. - Sv Zool.

Sv. Takt'Toür.la, Jernsparf.

IIab. in abboretis el fruticenis passim. Autumun migrat mense Aprili reveniens. Insectis, baccis seminiluss grat mense Aprilur. Nidulans in fruticelibus ora 6 glaucescentia que re bis per anmura. Cantillat dihudi-fadi, clanio

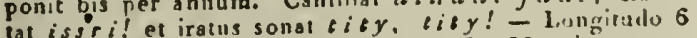
poll. Rostrum nigrunt. Iris fusco rufa. Macula temnopoll. Rostrum albo-linesta. Uropyerium grisen.brunnellm, inmaculatum. Gula sord.do alba. Abdomen el crissum albida, lioc maculis acutis fuscis. Lemiges fusci, ferrile gineo-maculati: rectricibus proximis a pice (plerumiur) maculis minutis albilis. Rectrices obscure-fuscr, miodie vero binx dilutius, marginibus ferrnginescenubus. Pelies incarnato-flavicantes.

G. 23. PHYLLOPSEUSTA **), $S_{1}$. Löfürls.

-) Hab. gener. prope Mlotacillas, sed colhs unguilus. quo brevioribus ef clenracerilius feliericis valde decerninalus. proverea reuige ":o fore lonsiculine 3:i, qui lorgissimus; pedilousque robustus, ungue postice ut in Mgueracilla. - Nomen getrar.

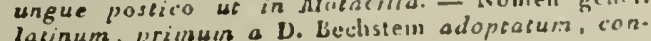
servavimus.

- Habitus fere Sylvix sed forma rostri al ilits if ol generibus aliis bene distificsus. Coput a'sib 

1. hippolais: cinereo-olivacea, subtus pallide-flava; alx culudaque equali fusce: mar. ginibus remigun interiorntn et rectricum la. teralium albis; peailous plumbeis.

Linn. S. N. 430.7 Fn. 248. (Diagn.) Molacilln. - Retz.

Fu. 237. Molacilla salicaria. Nilss. Orn. 105. Fn. 209. 12. Sylvis. - Sv. Zool.

Sv. HäslbocksLöfärla.

Hab. in sylvis el hnrris non infrequens. Reveniens circa medium Alaji abit aulumno. In nido inter rauslns antifiose constructo ubconoideo, corlice Betula allow rapyro sinnli circumdato, ord 5 rubrescenti-alba punctis reuisque obscure-sanguine is prastrtim in extrenitate nujore obscuriore ponit. Cantillat sonore penetrabile el inodulate. unde Garmani ean vocant Bastardnachtigall. Pellicit $d \ddot{a} c k, d \ddot{a} c k ; f i d h \circ i$, fidhoi! lestita clamical: $b \ddot{a} c k$, täck, täck! irala que: $\iota n c k-a k h y 0, t a c k-a k h y 0$, li $y$ ! - Longiludo $5 \frac{1}{2}$ poll. llostrum lusco-incarnstum, subtus lavescente-incarnatum. Iris rufo-lusca. Siria pallido-nava n naribus ad oculos. Palpebre pallide-nava. Subius pallide-hava: crisso tectricibusque alarum inferioribus pallidiuribus. Remiges obscure-fusci: primoribus tenue. secundariig latius margine exteriore luteo-viridibus. Rertrices obscure-fusca : extima pallidiore, tenue albn fimbiata. reliquis iutus albiclo extus pallide virescenti-marginatis. Pedes plumbei: unguibus griseo-fuscis.

$$
\text { G. 24. RHADINA *), Sr. Finärla. }
$$

depressum, ad basin rosti acute productum; caudia erutrata l. subbilarca; peáes graciles. - Sioul.

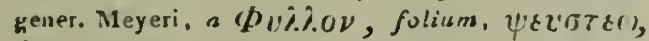
frustror derivatun, conservandum est, quando Asilus Bechsteini jaindudum est nomen genericum encomologicum.

-) Hab. gener. fere Phyllopseustx, sed corpore gra-

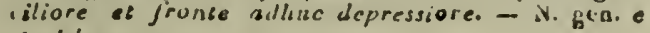
iudivos, grucilis, derivalum.
1. sibilatrix: fluvescente-viridis: superciliis luteis, fascia per oculos fusca; gula juguloque flavescentibus; abdomiue crissoque albis.

$f$. colore pallidiore.

Bechstein Naturueschiclite Deutscliands Ed. 2. T 5. peg. 561. Nilss. Ora. 108. 1. 7. Fn. 211. 13. Sylvia. - S2. Zool.

Sv. H visse I Fin ärla.

$H a b$. in Srecia australi rara, priesertim in fagetis $(D$. Nilsson). Nidulatur inter radices truncorum ad terram, ova 6 oblongo-nvala parva pallide-grisea rufo-fuscoquemaculata el strizta ponens. Cantin dju, dju dju-ipp mop sipp serr! - Longitucin 5 - pull. Ros!rum fusipp sipp serr. Caput el uropygium viridia naroscum. Iris arellana. Caput el uropygium ridiam flars, lincla. Stria a naribus supra oculos ad nucham lara, sed porée osulos palidior. Stria oculari fusca, pol, margine los latinr abscurior. Remiges nigu a ice tenue alexlerigre farescemte-virides: interioribus a pice tenue albidis et lectricibus mojoribus nigro-fuscis marg ne nanctis scente-viridibus. Ala sublus ad margnem navze punctis nigris. Cauda bifurca: rectricibis obscurid fuscis, exlimo margine exteriore pallido, celeris extus farescente.virid marginatis.

2. acredula ${ }^{*}$ ): cineren-viridis: superciliis pallide-flavescentibus; sublus albido-flavescens: flexura alarum sublus flava; pedibus luteo fuscis.

Linn. S N. 338. 49 Fn, 364. Retz. Fn. 352. Mo'acills irochilus. Nils Oin. Iug. Fn, $21 \frac{1}{2}$ if Sylvia trocbilus. - sv. Zool.

Sv. Skogs Fiubirla, Skogsknelt, Sparfhung.

-) Numen, quod Linuras varietati dedit, recinuimus, quis of erronelum et ineplum videbur speciel's ed quia co erronem denominatione signars. 
2 $\sim-$ 
Var. R. remigibus -9 apicibus albis.

Linn. I c. - Fn 265. Molacilita aciodula.

Hab. in salicetis et beculetis sat frequens, in hortis etiam occurrens, var $\beta$ ron:en rarior. Redux ad finem Arrilis abit mease Octibri. Nidun clausum introttu laterali rotundo construit is ipsa terma et ibi nva 6 aloa msculis volaceis ponie. Clamitar fict, fiit! et cantillar triste: di didy hy de hi tsin isia! - Praredenti sinilis ederrique macuniudine, piater caracteres indicaros tymen stria uculari fusca tantion ad oculos abeunce temporibus lisscescentibus, gula albula, margino remignn fuscaun exteriore olivacen, replitilinsquelascis, margine interiore pallidis, exterius o oliraceis, inunifesto diversa.

3. rufa: olivacco-fusca, subluts alhidat. favo imnixto: stupercilits llavescentibus; rcctricibus alarum inferioribus stramincis pedi. bus nigro-fiscis.

Lathain Index ornitlul. 2. p. 516. Sy!via. - Nilss. in Acr. 110lm. $1819 \mathrm{p.} 185$ t. S. Sylvia abietiaa. Fn. 2i6. 15. Sylisis. - Sv. Znol.

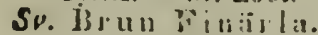

Hab, in abieris Nosregix non inlrequens supra $\mathfrak{N i}_{-}$ drosiam, in Sturdalen. Enderoen et Numinedalen; $D$. Nilsson. Insectis larrigrule victict. Mlifratoria. Cariat kipp kipp kipp-kjapp.kjapp, kjapp! tror.

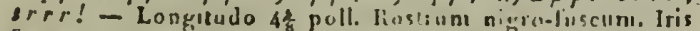
Iusca. Uropygium cune tectricibus aiaruin obscure seuin olivaces tinctuni. Nacula temporis fuscescence. La. tera colli peclosisque gricea luters immirtu. Firmues lu. sci: exterioribus extus tenue poliist gristo intepinritus olivaceo-marginatis. Canda subatualis: pontriribus interne et rersus aprcen extorne fuscis, teane albidu-iuarginet:s, extims utrinque niargine esteriore pallida.

\section{G. 35. REGULUS *), S*. Kongsfagel.}

-) Hib. gen. pracedensi valde approximat, sed ro. aro bene dircinciss of medius inter Paros ef Mdo. iscallicidas. - N. gen. Ihoji latinuin est.
1. cristalus: virescens su?,!n' albida: ver. tice crocer; remigifils fuscis balere externo flivo-virillib!!s.

f. vertice litico.

Linn. S. N. 55849 Fn. 262. Rotz. Fn. 258. Vinmm. Fu, 256. Mntscills Rie!nlus Nilss. Oın 1so. Fn. 218. 16. Sylvia riezulus. Si: Zool.

Si. Tofstiong sfägel, Fingelkung, Busklus. - N. Funlehonge, l'crunisma!l.

Hah. in sylvis hortiangue sat vu'paris, manens. Iasectis diversis. larvis oviatue eorutn el semill.bins rescisue: seininibus valo blassisw moritur. Jidum giobosum introita unice superton vel latersli sisb amulis pinosuat versus orientem labricat, nbi 8-rs nra ralde cubtusa, lare ur pisa loorens.a, vallide incarnata ponib. Pe:licit sst, tsit-tsit tsiss! - Longitudo 4 pu!l! unde inter aves mostras mum ma. Simillimus liliadine acredula sed macula ejus mogns in vertice, lines $n$ gra uthinutue marginals. guan fin lubitu crisialosine etigrit el demiltit, pratei nutas dictas mox crenuscitur. Tectrices alarum iusce, auicibus majorilm abis. lienciges fusci unargine $* x-$

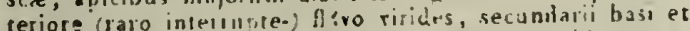
illtino apice albi. lieclitices fuscre extus viridescentomatginala.

G. sก. NANiUS *), Si. Lillärla.

1. troglodyles: ferrugincu-fuscu, nbsolcla nigro-undulata: remígibus cximis nigro-tessellatis, candia cunciforna lineis lounsersis fu. scis.

f. colore magris anfescente liscisque t1insvirisis obsuletionilents.

Linn S. N. 337. 45. In 26 r. Retz. Fn. 250 Miolacilla. Jainm. Lis. 252 Mulcita firsca. - Nilss. Oeu. su. Fil. 224. 5. Sylvia. - Sv. 200l.

-) Hab. gener. precesdentis sed corporo breviure el coudo plerumque surgentc. 


\section{-}


Sv. Pyssling Lillärla, Gådismyg, Tumme. liten, Tumlille. - N. Rindil, Musebroder.

Hab. in Provinciis oustralioribus, ubi hibernat, sat frequens; rarior vero ad septentrionem; hieme loca adiGicala hortosque frequentat, in sepibus lapidosis ot in fruticibus densis versatur agilis, caudarn érectam el alas dependentes ferens. Pellicet tsrr, tsro, tsesserre! et caurillat non insuave. Insecta, cunt larvis, ovis puppisque eorum, incerdum atiam bacce diversa cibum ei prxbent In cavis arborum, sub radicibus nidum ovale introitu laterali, extus muscis intusque plumulis crinibus j" collsiruit, ubi fem. bis per anuum, oro sub-roiundata alba punctulis subrescentibus ad extremitatem erassiorem ponit. - Longitudo circiter 4 poll. Corpu. subtus cineren-testaceum, nbsolete liusco-undulatum : ro. stro nigro-fusco basi subrus flavescente, superciliis albis, remigibus internis fuscis. Pedes fusci.

\section{G. 27. TITIZA *), Sv. Qritcerärla. *}

I. schonobcena: capile nigricarte, strits griseo-olivaceis strinque albida supra oculos; dorso griseo-olivacco maculis fuscis; uropygio sordide-ferrugineo; subtus ferruginescentealba.

Linn. S. N. 32g. 4. Fn. 246. Retz. Fn. 23/4. Motacilla.Nilss. Orn. 107. Pn. 1. 221. 17. Sylvia. - Sv. Zool. Sv. Säfstigs Qvitterärla.

Hal. locis paludosis inter calanarias et equiseta pas$\operatorname{sim} \boldsymbol{D}$. Leche; nigratoria; Insectis var.is victitans. Pel-

-) Hubit. gener. adproximat G. Sylviato, sed alis bre. vilus rotundatis pennis arcualis, cauda satis longa rotandata, unguibusque magnis scutis curvatis beno discinclus. - N. gener e voce graco I'iti$\zeta \&(\nu$ eilissare. licit: hidd hidd! cantillans noclu. - Longitudo 5 poll. Rostrum nigro.fuscum, subtus basi pallidum. Iris castanea. Fascio superciliaris nigra striaque a noribus supra oculos versus nischam albida et ultera per oculos fusca, pone oculos latiore rufescente. Remiges lusci: uhimus extus albido-nıarginatus, interiores cuni tectricibus griseoolivaceo marpinati; tectricibus inferioribus albidis luteotinctis. Cauda rotundatn-cuneiformis : rectricibus fuscis apice rotundaro, pallidius marginato. Pedes sordide-llavescentes: unguibus pallicle-!'uscis.

2. Ligluffooti ): griseo-fusca subtus alba pectore, lateribus crissoque ferruginescentetincla.

Lınn. S. N ed. Ginel. 1. 992.196 . Motacilla arundinacea. Nilss. Orn. re6. to 6. In. 1. 22j. 18. Sylvia arundinacea. - Sv. Zool.

S2. Light foots Qvitter ̈̈rla.

Hab. ill Suecia mediu rarissime. Vescitur insectis baccisque. Nidulatur culunis gromineis inter 5-4 culmo urundillaceos. Cantillat egipgit el clarnitat absch! I.ongitudo 5 poll. Rostruin ninru-fuscum, subius lnteoincainatum. Color superuus iu olivaceum vergens: ca. pise obscutiore et 11 ropy pio magis ferrufinescente; a noribus supra oculos slita athida. Gula alba. Remiges obscure-fusci, marginibus pollidinribus: tectricibus inferioribus sordideoulbis. Recirices obscure-fuscx lateribus pallidioribus, pice fere albido-marginntus. Fedes plumbed: unguibus praserrion postice robustis, compressis.

$$
\text { G. 28. SYLVIA **), Sv. Skcgsärla. }
$$

-) Quia persuasissimum habuimus Turdum orundinaceuil Linn ad motacallaidas numerari debere. adeoque untiquam ejus dinominationem specifiram esse servandrim, speciem hanc Inventoris (Philos. Transoce. Vol 75 p. I. p. 8. f. I) nomatie disjun-

-o) Hubs. gener. fure precedentis, sed remigibus 5, Jongissimis, a broviore primugue adhus breviore; 
1. nisoria: fusco-cinerea sublus alba cinereo-undetala: rectricibus firscis, lateralibus 2 iutus ad apicem inacula albe.

Nilss. Orn. 95. 1. 5. Fn iS3. 3. - Sv. Zool.

Sv. Sparfhöklik Skogsärla.

Hab. in alnetis Scanize ratissime ( $D$. Nilsson). Ni. dificat in ruticibus. Victitat insectis. Ivolitat perpendiculariter in acie more Alaudariom et cantat fere. ut $S$. Curruca. - Longitudo 7 poll. Roserume fuscum. Iris aurantia. Remiges fusci margine dilatiores, a picibus secundariornm et iectricum nigiornm albidis. Rectrices $x$ quales cinereo-fuscre: -1 paulo bievioles introtsum verins spicen dimidisto a!bx, raro tantull apice albx. 2-2 maculs magna apicali alba, ceterx leviter albo-termiaulx incermedis inmaculatis exceptis.

2. plitomela: fuscescens sublus grisen. alba: remige secundo quarto longiose, caltdaque fusco-rufa.

Nilss. Fn. 180. 2. Oru. 94. S. Luscinia. - Linn. S! N. ed. $G$ mel. $9^{50} .1$. Motscilla Luscinia $\beta$ major. Sv. Zool.

Sv. Sång $S$ kogsärla.

Hab. Scanix secundum observationes D:i Nilsson. Mores cum sequente convenimut, a quo, quanvis difi. cile, distiucts est prater notas indicatas colore sordidiore, magniludine najore (6⿳亠丷厂巾-7 poll.), et canlu, quam

Cel. D. Bechstain ") ita exprimit: gia-gy, $\boldsymbol{g} y, g y$ !hagoi, lagoi, zii ziiziizi.-gergegegegeGe,h-zickazerrrrerrr-4oa, goigoigoi-gi. zickazickazicka. Davitt, davits, davist!

cauda pedesque mediocres: sarsis iamen gracilibus digito intermedio longiore, unsue postico digito posteriore bieviore, curvato cumpresso. No. men generis latınum Brissoni $a D$. Latham us genericuin acceptum.

-) Bechsteins Nuturgescls. d. Sinbenvögel 5 Aufl. s. S5o. ovavavavavava , ,gockörk, gockörck; ge: den, geden, geden, geden, gui-Goignigoi goi girrrrelhllipp, philipp.phılipp! golka golaagolkagolk. IIia, giagiagiagla, glack glock flockglockglockglock.Geágea gea gi! slerk, srleerck, sherketz-goi, g deagaga gägi. Heid heid heid heid $h i$; vol, da, da! volda, da! geigeigei girreirr Hoi, ge gegegei-hoifoi! zerrrrrroiz. - Pelicil: $/ h$ i glock arrr! vel davist inkop. - Longirudu 65-7 poll. Iris rufescens. Characteribus indicalis el cantione sequeire facile connira.

3. luscinurs; rufesccns, sublus grisen-alba: remige secundo quarto bitviore, caudaque rufesicente.

Lirn. S. N. 528. T. Fa. 244. Recz. Fn. 252. Molacilla.Nilss. Fn. 1>6. 1. - Sv. Zool.

Sv. Näktergals Skogsärla.

Hab. in fruncetis ad rivnlos per annum rorior; nunc temponis vix extı Gort!andian. boream versus. Venens sub initio mencis Mnji nidilicat in frul cibus, rope tetramer ponit ura5.6 sordide olisaced, quare sirciter dies $14 \mathrm{mare}$

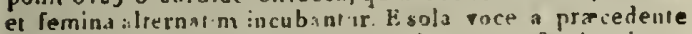
distincla est. Pellicil vittiker r! et mas feinina incubaule camtillat suarissime sunu a D. Bechstein ") exprimato: riu biu tiu.spe tiu qua ...tio tio sio tio sio tio tix; qusio qustio qusio qutio,...zquo zquozquo zquo: bii tzii

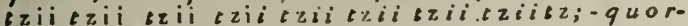

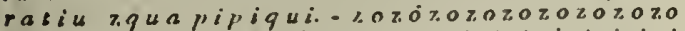
zozozozirrhading! tsisisi tsisisisisisisisi, zorrezorrezorrezorrchitzizb $b z a t n t z a t n$ batn tratn bzatn izain zi,-dlo dilo dlo dlo dlodlo dlo dlo dlo dlo:-quio ter rerrerre itr. lialiilitlii.lyly ly ly il lilili. quio didl liliilyli. - ha Eiirr giirrquipin! gui quı quiqui,qiqiqiqi. gi fs gi gi: gulloull gollgoll gia hadabui. quigi horr las dia

-) Eeclusbins Naburgescls d. Stubonvïgel 3 Auf. 535 
diadillsi! hezerezerezerezrezezerezeze zezezequarhozehoi; quia quia quiaquia quia quiaquiaquia li: qiqiqialo io io ioioioqiliily li la läla lo lo didlio guia higaigaigaigaigaigaigaigai $i a g$ io guia iquior ziozie pi. Hec emodulatus conticescit jul jis evolentibus. Insectis, ovis formicarum et baccis $\mathrm{Li} i$ bis et Sambuci victitat migrans ad finem restatis. - Longitudo ejus 6! poll. Iris obscure-fusca.

4. atricapilla: supra obscurius subtus pallidius cincrea : pileo nigro.

$f$. pileo obscure ferrugineo.

Linn. S. N. 532. 18. Fn. 256. Retz. Fn, 244. Motacilla. - Nilss. Orn. 96. Fn. 185. 4. - Sv. Zool

Sv. Svarthät $\mathrm{Sk}$ (igs ärla.

Hab. in hortis et sylvis non frejuens. Migrans a tumno revertitur ad finem-mensis $M$ aji. Insectis, Jarris et baccis diversis rictitat. Nidificans in fruticibus densis. ova 4-6 lutescenti-slba ferrugineu-fuscoque moculata fem. ponit. Pellicit $t a c k$ ! et anımo irato clamat dieb! dieb! - Longitudo $6 \frac{1}{2}$ poll. Rostrum nigricans, subtus glaucescens. Iris brunnescens. Supro ulivacen.tincia. Gula, abdomen el crissum albida. Remiges fusci extus nlivaceo-tincti, primores 2 extus tellue albo-marginati. Cauda equalis; rectricibus acuminatis luscis olivaceo-marginatis. Pedes plumbeo-fusci. Dorsun feun. griseum olivaceo-tincium. Gena gulaque pallide cinerex. Pecius \& Hypocliondria pallide-grisea. Abdumell album, rubrescente-tinctum.

5. salicaria: cinelco-fusca subius cise. ren-albirla: pectore levitcr fulvesccnte, palpebris allsis, pedibus plunbeis.

Linn. S. N. 530.8 . Fn. 249 Mulacilla. - Retz. Fn. 256. Mntacillo Hippolais. Nilss. Oı13. 97. Fn. 387.5. S. hortensia. Sv. Zonl.

Sv. P'il Skogsärla.

Hab in sylvis pass.no, frequentior tamen in provis. eiis australioribus; migrat .. - dio Sept. redit me.'io $M$ in. Vescitur proserlim lasvis \& baccis. Nidilicat in Irutic:- bus densis epinosis potissimum Cralægi oxyacanthes, ubi ora 4-5 cinerec-alba: maculis plus minusve fusco-nlivaceis. Cantillat suave; pellıcit $t \& a, t s a$ ! stridut $g \ddot{a} a ̈ c k$, $6 \ddot{a} \bar{a} c k$ ! et clamosem terrirus rollit t a $c k$, c a c $k$, t a ck! - Longitudo circa 6 poll. Rostrum fuscum, subtus pailidum. Iris rufescens. Color supernus in olivaceum vergens. Abdomen et crissum alba. Remiges fusci, apice renue pallide tincti margine exteriore nbscure cinereo, tectricibus inferioribus dilute farescentibus. Cauda equalis: margine rectricum exteriore apiceque.albido-tinctis. Ungues pedum griseo-fusci.

6. cinerers * : cinereo fusca subtus albi. da: remigibus latere exteriore ferrugineis; rectrice extimo extus longitudinaliter oblique secundaque apice alba.

Linn. S. N. 330. 9. Fn. 250. Retz. Fn. 258. Motacilla Sylvia. Nilss. Orn. $38 \mathrm{Fn}$. 188.6. - Sv. Zool.

Sv. askgrai Skogs ärla, Skogsknctter, Mes. $\mathrm{Hab}$, in sylvis sat frequens, hortos interdun invisens. Migrans autumno revenit medio Majo. Nidificst in fruticibus frondosis densis et ova 5 viridiscenti-alba punctis oliraceis fuscisque ponil. Insectis larvisque victitat. Ad vesperem usque serotinuin jucunde cantillat, pellicit is $a$, - is a! et irata vociferatur $g \ddot{a}, g \ddot{a} !-$ Longil. 6 poll. Rostrum fuscum subius pallıduin. Iris pallide brunnea. Caput supro et cervix cinerascentia. Dorsum cinereo-fuscum rufescente leviter tinctum. 'Temporum macula dosco concolor, sed latera capitis pallidiura. Remiges fuscl: primoribus 5 margine exterture tenuiter albis, socurdariis cum tectricibus majoribus late ferrugineo-marginatis. Gula et abdomen alba cetero subtus albo-rufo-

-) Contendenti D:o Nilssen, Linnaum et Retrium citato nomine hanc speriem signijicavisse haud as. sentire possumus. Quas hi aucbures dederunt. no. ta caracteristice veribatem sententist nostia pro. Lant; atcamen D.o Nilssoll, cujus scuc'io hirum specierum cognitionem altigunus clariorem. plurinz debuinus. 
1 
acentia. Alx ad basin subtus albre macalis parria fuscis. Cauda refualis: rectricibus luscis exfımn porte breviore cineraicente macula oblonga $\&$ apice secuindze alba. Pedes fusco-flarescentes: unguibus cinerascentibus.

7. curruoa: rufescenti-gr isea subtus alba: capite cinerascente inacula temporum obscuriore; rectrice extima latere exteriori margineque apicis interiori alba.

Linn. N. 329. 6. Fn. 247. Retz. Fn. 235. Moracilla.Nilss. Orn. 99. Fu. 192. 7. - Sv. Zool.

Sv. Kr uk Skogsärla, Ärtfủgel.

Hab. in sylvis loortisque haud rara el ibi in fraticibus presertim-specierum hibis nidificat, ora 4-5 albe $\mathrm{ex}$ cremitsce obtusiore maculis cierulescentibus al brunneis ponens. Vescitur insectis, lorvis et baccis non minus quam ponens. Vescitur insectis, lavis et baccitur ad fiuem menpisis. \&c. Migrat medio Sept. et revettitur ad fuen mensis Maji. Cantat longesuevergue; pellicit $k l a p p, k l a p p$ et s $a$ ssa! - Longitudo $5 \frac{1}{4}$ poll. Rostruin nigricáns, el is a ssa:- - Longitudo Iris extus albida, intus rufa. a Caput cinoreum, ferissime fusca pone oculos latiore et obbus per oculus cinereo-fusca pone oculos latiore et pectore. tertricibas alarum inferioribus crissoque. in rufectore. rertricibas alarum inges et tectrices mojores fusci, rufercente.grigeo teque marginati. liecirices fuscx, exina paulo breviore, pallidiure, latere exteriore et marpine interiore ad apicem alba, (raro dimidiaso oblique glbida proter lineam fuscam secundun rachin), socunda apice lbo-marginata, medix bina albido- relinux rufescente-griseo ienue marginatz. Pedes plumbeo-fasci. G. 29. PHECA *), Sv. Qvickärla.

x. rubecula:

- Ilabit. zener. fure Sylvire, sed pedilus Eraciliorilus, ungue portiro mediecri, curvato Niumen genericuin liechsteini, Ficedula, ut specijficum aius generis, ineptuin est, unde hoc ogrecé $\Phi$ aıkOs (•gilis) elegimus.
1. rubecula: sordide olivacea: 'fascia fiontali per oculos, collo autice pectoreyte fulvorubris.

Linn. S. N. 537.45. Fn. 260. Retz. Fn. 249. Hamm. Fn. 253. Mutacilla. - Nilss. Orn. 100. Fn. 193. 8. Sylria. - Sv. Zool.

Sv. röd Qvickärla, Rotgcl, Rödbrösta, Rödhake. $-N$. Rödhals, Rödkielke, Rödfinke.

Hab. in sylvis hortisque sat vulgaris Insecta, larva, beccar prasertim Eranyni viclus ejus. Nidum clausum. introitu isntum unlco, fabricat in terra, in siruibus lapidun rel in cavis arburum, ubi ova 5 sordidealbs, pundum rel in cavis ascomitate obtusiore confluentibus, bis ctis rubro.fuscis exiremitate obrusiore confluentibus, bis per annum ponit. Abit autumno serotino ellicil: sisi, jamdudum, mense Mario. sisisri! - Longitudo 5t poll. Rostrqun nigro-fuscura Iris fusca. Pectus inferius, abdomen et crissum alba. Remiges of rectricés acuminati fusci latere axteriore sordideolivaceis tectricibus majoribus apice maculis, parvis harescentibus, inferioribus fuscescentibus luteo immixto. Cauda aqualis. Pedes grisęo-fusci: unguibas abscurioribus. 2. suecica: cinereo.fusca: gula carulea, cauda basi lerruginea, apice vigricante.

$f$ gula albida: stria longitndinali ntrinque ad latera nigra, jugulo stuis 2 cicruleis, pedibus totis incarnatis.

Linn. S..N. 336. 37. Fn. 250. Ratz. Fn. 248. Motacilia. Nilss. Oru. 103. Fu. 206. 9. Sylvia. - Sv. Zool.

Sr. Sreusk Qvickärla.

Hab. in salicetis et alneris Nordlandixe et Lapponix; Hab. in salicetis et alneris Nordad liossiam. revenit migran outumno per Finlanilian ad lipssiam, revemit

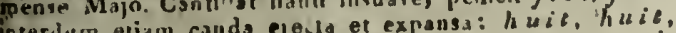

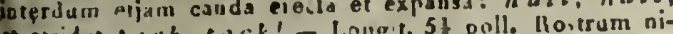
a stridet cack, tark! - Lang't. 5 t poll. Thoitrum ni-

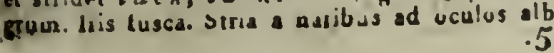



pora fusco-rufescentia. Collun antice a gula ad sternam nitido creruleun fascia nigricante terminata. Pectus anrerius ferrugineum. Abilomen ulbidum. Crissum alboferrugineum. Rentiges fusci cillere.)-fusco masginuti. Rectrices a basi ad niedium ferrugcinex, celero nigricantes intermed is vero fuscis. Pedes incsrnati: digitis nigricantibus.

3. plunenicura: cæruleo. cinerea: fronte alba, gula juguloque nigris, pcclore cauclaque rufis, rectricibus interncdits finscis.

$f$. rufescenle-grisea: gula abdouninerqe albidis; pectore sordide-ferrugincescente.

Linn. S. N. 535. 34, Fn. 257. Retz. Fn. 245. Hamm. Fil. 255. Moracilla Phoonicurus (in.) Linn S N 335. 5 j. Fn. 259. Retz. Fn. 247. Motscilla l.ritharus (f) Linz. Fn. ed. 8. 227. Motacilla l'ilys (junior). Nilss. Ora. Xor. Fn. 202. 10. Sylvis. - Sv. Zool.

Sv. rödstjertsQvickärla, m. Rödstjert, $f$. . Rödbuk. - N. Blodfugl, Röilstjeft.

Hab. in sylvis horlisque sat frequens. Migrat ad isnem Sept. et redit ad Gnen Aprilis. Nidilicat in sepibus et inter lapides, interdum etjam in partetibus et ibi ora $5-8$ pallode carulescente-viridia ponit. Pellicit: $l t u t:$, huit, ls uit, dit, dit! et terrilus clantirat: huit he ke! huic ke ke ke! - Longitudo 6 poll. Rostrum uigrum. Iris nigricans. Cetpro per notas indicatas baue cognita.

4. Nilssoni o): obscure-cinerea: fuscia frontali, gula, jugulo et superiore pecturis

- Nomen Títys a Scopolio adhibitum nec Intinum nec. grecum non retinendum putainus, quare no. men viri de Ornithologin opteme nerth ef qui hanc et alins species hujus natuors maximn sagacia illustravit in Fauna scandwnavaca conser vandi occasio sese grata oblublit. parte nigris; rectricilons rufis intermediis 2 fuscis.

$f$. abdomine griseo variegato; remigibus fuscis extus albido-marginatis.

Nilss. Orn. s02. Fn. 207. II. Sylvia Titys. Sv. Zool.

Sv. Nilssons Ovickärla.

Hab. in Uplandia rarissime (D. Excell. Com. Trolle Wachtmeister). Victitat ut congrneres prxecinue insectis. Nidificat inter lapides in domibus ora 6 albida ponens. Pellicir: fit, fit, fitsa! - Marnitudine prioris, cui similis, sed a yua tamen differ procter cliaracteres dictos colore superine obscuriore, pectore cinereo-nndulaso, hypocliondriis cinereis, abdonine tantum medio albo, crisso luteo-rufescente, margineque renigum cinereo.

\section{G. 3o. SAXICOLA ${ }^{*}$ ) $S_{v}$. Sténsquätta.}

I. rubetra: nigricans: marrinilus pennarum grisco-ferrugineis; macula ilarum striaque supra oculos alba; jugulo pectorcyuc lu. ten-rubris.

$f$. obscure-fusca marginibus pennarmu brescente-griseis, stria supra roculos ruberse cnto. alba, macula in tempore fisco-rufa, macula. que alari miture.

Linn. S. N. 352. 36. Fn. 255. Retz. Tn. 245. Jolacilla, Nilss. Orn. 92 Fil. 169 2. - Sv. Zonl.

Sw. rödlett Sténsyvälta, Huslistritia.

Hab. inter frutices minus rara, ulji hat d longe a terra nidıficht, ova 5 pallide-carulea immaculata pnueus. Migrat aulunuo et ad linem iprilis revestisur. Fes, isur

-) Hab. gener. pracedentilus opproximal; sed semi gibus 2 e 5 longissimis primorue lirevissimo; "all da breviuscula aqualis. Nomen well. liectosisin. a verbis lacinis: saxunı ef culo, forliollim. 
insectis. Cantillat mane vespereque non iujucunde; pellicit git! concrepat es a. - Lonyiludo 5 noll. Rostrum nigrum. Iris griseo-finsca. Fascia !ala ab anguln on is sub oculis arl lalera colli nigra, inlerdum rnfo alboque im. mixlis; in fem. nulla Gula alba in lineam ad Intera ju. guli descendens. Pecrus inferius el abdumen slbs : hy. pochondriacrissnyue $h_{d}$ vescenle-rubro levier tinclis $V_{10}$. pygium griseo-ferrugineum maculis nigris. Kemiges ob. scure-fusci, exlus grisen-lerruginei, inlus albido-marginali, primores basi albi. Rectrices nigro-fuscre, rallide marginale: intermediis 2 basi, cateris plus dimidios superione albis. Pedes nigni: femorbus albidis inaculis par. vis nigricausibus.

2. OEnonthe: cana subtus alba: fionte supra oculos noopygincue albis, fascia per oculos nigra, canda alba npice nigra.

f. minor: macula in tempore parva, ob. scure-sufescente, capile dorsnyue cinereis $1 \%$. bress:ente-tinctis; gula, jugulo pectoreque surd:de lerrugrinesconte-Iuteis.

Linn. S. N. 332. 15. Fin. 254. Retz. Fn. 242. Hamm. Pin. 254. Motacilla. - Nilss, Orn. 93. Fn. 167. 1 - Si. Zuol

Sr. cgenttig Strusquät is, Stcingylp: Stendolp. - N. Steendolp, Stecusqvictic, Stcengylpr.

Hal, in lapidosis ubique migratosio, reveniens cum

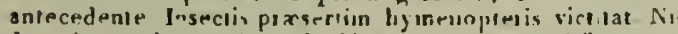

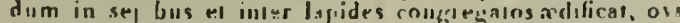

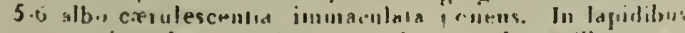

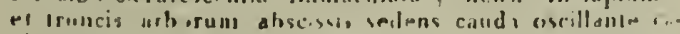

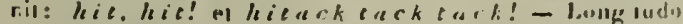

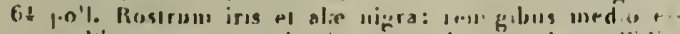
lis politite grisen-masginalis, secuncalus apice palidis,

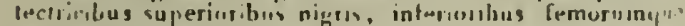

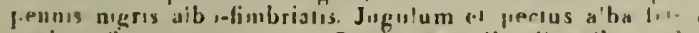
rugines-flaresceate linclu. Canda pius dimidia alba. II picem nigra : rectricibus tantum a intermediis a basi ad tertium partem albis. In $f$. macula fronlalis fosciaque deficiun; ala fusca.

\section{Nat. 9. LANIIDES,}

G. 3т. LANIUS *), Sv Warfågel.

1. Excuhitor: canus, sublus albus: fascia per oculos nigra; alis nigris maculıs binis albıs; cauda nigra lateribus albis.

$f$. colore sordidiore; undulis pectoris cane. scentibus tenuibus.

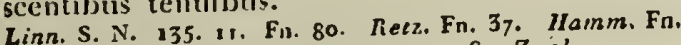
87. Nilss. Urn. 33. Fn. 144. 8. - Sv. Zool.

Sv. egentlig Warfäg el. - N. Klavert.

Hab. in sylris minus l'requens; sonu: Truicrua ihr gihr mox dignoscitul, excubitor accipitris ad. venientis avibus est parvis. Mures, avicule, el insecla renientus avibus est parvoribus nidulans $1 \mathrm{em}$. ova $6 \mathrm{pal}$. sunt victis ejus. In alboribus nidulans lemil et dies 15 lide-grisea viride- et obscuro-machlala rorii et dies fovet. - Lnngitudo 9 poll Iris fusca. Fascia nigra rostro per oc
guibus nigris.

2. collyrius: cinereus subtus roseo-albus: fascia per oculos nigra, dolso biunnescente; cauda nigin lateribus versus basin albis.

$f$. fusco-ferruginea, nigro-albidoque-undulata, subtus Juteo-alba fusc:o-undulata.

, Fn. 3s. 1. 2. f. 81. Re:z. Fn. 38. Ham. Orn. 32 . L. collurio. Sv. Zool.

-) Habit. gener. madius inter Corvidas et Motacillx.

) Habit. gener. mudius inter Corvidas ot Motacillxbus: remige lrevi, 3 et 4 longissinis; tbiis digito intermedio longioribus. - Nom. gea. labin:" vecuscum Gesneri. 

70

Sv. lîngund Warlâgel, Törnskata. - $\pi$. llantvirh.

nazb. in sylvis sat frequens; mense Sert. migrans. Majo raventir. In lruricibus nirlulans lan. ova 5 lanescenteaiba maculis luteis er grise 15 bis ponit per anuun et dies 14 incubat. Piater victum prusis eljam amplibiis victitat. Sonu: $g \ddot{a} c k, g a ̈ c k, g a ̈ c k, a t s c h$, atsch! pellicit. - Per notiones dicias ab antecedente sutticienter diversus. Longitudo 6.7 noll. Iris tiusca. Fascia niugra a naribus per oculus ad latera colli. Pelles nigri - Aumen specificum auriphum: Collurio, nec latum, ne: græcuin mutavimus.";

Nat. 10. TURDitIES,

Div, r. digitis externis longilndine media unitis:

G. 32. ORIOLUS **), $S_{v}$. Guling.

y. gallula: flavus: loris remigibusque nigris; rectricibus nigris, latcralibus, apice flavis.

$f$. luten-viridis subtus sordide viridescens: striis fuscis, remigibus fuscis; canda olivanca, apice flava.

Linn. S. N. 160. x. Fn. 95. Coracias Oriolus, in act, Holın. 1750. 127. t. 3. f. 5. Ampelis hava. Retz. Fu. 4!?. Sparrm. Ornithologia fig. m. et $f$. Nilss. Orn 44. Coracias galbula, Sc. Fn. 155. x. - Sv. Zool.

sv. egentlig Guling, Gylling, Sommas. guling, Gultrast.

-) Lanius rufus Retz. Fn. 59. in Scandinavia vix inventus, unde cum heic adoptaro nequinus

-) Habir. gener. Turdi magis quam Coracix in arrtiore propinquitate est; ala mediocres: remige nrimo brevissimo, $3: 0$ longissimo. N. gen. latinum esi antiquum.
Hab, in Westroboltnia rassima, an ramen ibi nidulatilr: in Finlandia vern pracipue in Carelia Savolaxiaque minus rarus. Veniens mense Najo, migrat in crassituInter ramulos arborim nidun lulgiludine dine 3 poll. filis attificiose suspendit, in quo ova fam. podide alba extremitate obtusiose finscolmaculala fem. prio nit et cum mare 14 dies incul,at. Pullinent. Insecta, colorem summ splendentem primum nbtinent. Insfoc, larva et baccre, praseltim cerasi, cibus ejus sunt. Longitudo ejus 9 roll. Rostruin pailide-lusctumbei.

bra. Color corporis flavis nitens. Pedes plumbe

G. 3.3. TURDUS ${ }^{*}$ ), Sy. Trast.

1. viscivorns: olivareo-fuscus, subtus fla. vicante-albus: maculis pectoralibus triquetris abdominis hierumquc transversis ovalijus uigris; rectricibus i cextimis albis.

Linn. 3, N. 29r. I. Fn. 216 fietz. Fn. 201. Hanim. Fn. Nilss. Orn. 82. Fn 227.1. - S2: Zool.

229. Nilsorast. Björktrast, DubbelkjamsS2. Miste rer, hiansfiugl.

Hab. in sylvis non infrequens, hybernans in regioniThenelibus Scandinavia: major, lonbus allstration gitudine 12 poll. - Arstate insectis rermibuch Visci ulli, a quibaccis victitat, inter quas etjan bacesbium Turdus sibi bus viscum proparatul, unde provesbium cantillat et pelipse malum carat. Primo vere jucunde canticat, nbi fell. licit $I i s-r r$. In ramulis arborum altis nidife rifis nonit, ova 3-5 viridescenti-alba, maculis punctisque rins poxtudunt. e quibus pullos post is lies aiternatin pallidius: latere Iris obscure cástance. Dorsuin postice palo, linea per ocucapitis collique luteo-albu [usco-macularibus, apice superilos albicante; tectricibus alarum inferioribus, apice superiorum majorum, alisque subius albis. Pedes sordide

-) Hab. gener. antecedenti approximat; alis mediocribus: remige lrimo fere nullo vel orevi, gue vel 4 longissimo. Ni. gener. latinum. 

2. pilaris: cupite uropygioque cinereis, dorso castanco, tectricibus alarum inferioribus albis; rectricibus nigris, extumis margine interiore apice albidis.

Linn. S. N. 20r. 2. Fr. 215. Retz. Fn. 203. Hnmm. Fn. 228. Nilss. Orn. 85. Pn. 250. 2. - Sv. Zool.

Sv. Boll Tra st, Kramsfigel, Vintertrasl, Sü̈. skata, 13jörkdrossel. - N. Fieldtrost, Graa. trost, Nordenvindspibe, almindelig Krams. fingl.

Var. $\beta$. similis var. $\alpha$ sed capite colloque albis: pennis quibusdam fuscis tectricibusque alarum pennis 2 albis.

Thunb. in acl. Holm. 1808 1. 5. T. riscirorus var. Nilss. l. c. -

Hab. per totam Scandinaviam vulgaris, sed var. $\beta$. sarior in Ostrogollia $(D$. Westringl. Aulumno enigrat aul ad regiones ausiralinres vel exira Scandinaviam. E lumbricis, insectis it baccis gregation vivunt el nocludormiunl uno eorum vigilaule, qui dornientes suscilal cum scha schaschark. qu' snno eljam pellicit; zinzilutal: $f u i g u i$ In arbollbus pinetis nidulatur, ova 1.5 pallide-grisea maculis obsolol's fiscescembus ponens. Precedenti similis, sed yraler characteres indicatos, præsertim slalura minnre, longitud ne tallum ro poll., ro. stro favescente apice fuscn, linea oculari rulescenti-alba, iride fusca, dorsn ecapulistue costaneis, maculis pecio. ralibus longitudinalibus nigro-luscis, medio peclore abdomineque albidis dislinclus.

3. mnsicus: brunnescenti-olivaceus sub. tus albicans; collo antico fermginescente: maculis obcordatis fuscis; gula albida; remigibus hasi intus obscure croccis.

Linls. S. N. 292. $4 \mathrm{Fn} 217$. Retz. Fn. $202 \pi / 1 \mathrm{amm} . \mathbf{F a}$. 23̈r. Nilss. Orn. 83. Fn. 234. 3. - Sv. Z.ool.

Sv. M usik I' a s t, Taltrast, Fjällstare, Krams. fågel, Klädra, Klera. - N. Sangtrost, den Norske Nattergal.

Hab. in pinetis nun infrequens, oulumno provincias ausiraliures obeuns. Nidum globosiam e muscis in arboribus edifical el hunc stercore buvinn l. argillo illinit, ubi fem. ova 3-6 glanca punclis fuscis punit, qua post dies 16 allernalim excludunl. Vesperibus reslatis e summilale asborum cantus ejus Luscinix aliquonium zqual, non dissimiluer sonalls, secundum observanres germanicos: Davil, davil, drej rössel für eine kanne-prosit prosit kottenhans, kwehdieb! pellicit: zipp, zipp; lerritus clamitat: $j a c k, j a r k$, jack, zipp. zipp. - Similis nrecedentibus, sed longiludine lanlum $8 \frac{1}{8}$ poll. lines oculari cum orbita oculorum fulaque lulescenti-alba, lateribus capitis striis punclisque luirscenlibus, collda dorso concolore, irideque brunnea, Moribus vicluque illis similis.

4. ilincur: brunnescenti-olivaceus: stria a naribus ad nucham lutescente-alba, laleribus colli macula lutescente, liypochondriis alisque subtus ferrugineis.

Linn. S. N. 292. 3. Fn. 218. Retz. Fn 202. Hamm. Fn, 251. Nilss. Oin. 84. Fn 237. 4. - Sv Zool.

Sv. Trojas Trast, Björktrat, Rörlvinge. N. Nattvakke, Maaltrost, 'Tiletrost.

Var. B. tolus albus, rufescenti-nebulosus: hypochondriis alisque subtus rufis.

Nilss. l. c. - var. $\alpha$.

Hab. in pinelis ininus frequens, var. $\beta$. tamen rarissime, migralorius. Anlecedente adluc minor, longiludine lantum \& poll., a quo linea oculasi. hypochon. dris alısque sublus ferrugineis sufficienter diversus. Iris brunnea. Caulillat minus jucunde: try try try try try try $t i$, allectal: $\varepsilon_{a c k} g \sigma_{c k}$ el stridel inlesdum: st. st. Pedes griseo-luiesceules.

5. torquatus: nigricans: pennis alamus \& corporis subtus albido marginatis, wacula 

cirea partem pectoris superiorem semilhunari allit.

f. colore sordidiore maculaque collari fitsecescente.

Li.in. S. N. 296. 25. Fn. 228. \& m., $\rho$ f. Retz. Fil. 207. a $n$, 3 f. Hamm. Fn. 254. Nilss. Orn. 87. Fn. 240 5. - Sv. Zuol.

Sv. Ring'l'rast. - N. Ringtrost.

Hlal. in sy!vis monsosis rarior (D. Leche). Migrans mense Ocinbri severtitur $A$ prili. Nidulatur in arbusculis. Cantitlat tempinge vernali nimme unjucunde; pociteratur: cack tack tack! - Inngirndo in. 11 poll., $f:$ paulo minor. Rnstrum m. Juleum apice el basi supra higicans, $f$. Cuscum basi sublus sordide-favunı. Iris caslanea. MacuJa semilumaris albido est valde pulclura.Reniges el teclıices majores ning-fusci, exlus griseo-abn-marunati lecirices obsuse acumillate, extima ceteris alıju: $1111 \mathrm{~m}$ brevior, apice tenuiler ubo narginala. Pedas m. nigro-fusci, $f$. fusci. - Femina procul dubio var. $\beta$ Linn. et Retz.

6. meruln: ater: rostro margincque palpebraruin filvis.

f. uigricans: rostro intus flavo.

Linn. S. N. 295. 22. Fn. 220. Retz. Fn. 206. Hamm. Ftr. 232. Nilss. Orn. 86. Fn. 242.6. $-S_{v}$. Zool.

Sv. enslig 'Trast, Svarttrast, Koltrast, Soltrast, Solsvärta. $-N$. Sorttrisst, Soclsort, Sxisort.

Junior: supra nbscure-fuscus: capite, collo, dorso antice cum maculis subtus ferrugincis.

Var. B. capite allon maculis rarioribus fuscis, corpore nigro maculis unajoribus albis. Nilss Orn. - var. $\alpha$.

Var. $\gamma$. totus grisiore fuscus.

Nilss. Orn - var. $\beta$.

Hab. ubiqua in sylvis solitarius, varietales tamen rariores, et in fruticibus aliquol pedes altis nidulatur, ora
4-6 grisco-viridia maculis striisque brunnescentibus bis per annun: pouens. Clainitar davic, hans dobit, eljan interdum continuo sonat isitserp, tilck, $t a c h$ ? denigue cantillar jucunde. Characleribus indiratis bene ccälloscitur. Jris lusca. Longitudo yo poll. Pelles nig̣ri.

\section{G. 34. ICHLA "), Sv. Drossel.}

I. rosea: rosea: capite alis caudaque $n$ i. gris, occipitc cristato.

f. fusca ubi m. niger cristaque minorc.

Litrn. S. N. 294. 15. Fn. 219. Rietz Fn 205 Nilss. OIn. 88. Fn, 3. 245. 7. Turdus roseus. Sv. Zool. .

Si. rosenrö̈l D) rossel.

Hab. Lapponix rarissime (D. Adlerheim.) el Dalecarlize (D. Biom). Migrat inense diff. jer terras orientales, el in Asia occidentali, "bi grylins migrarosios devorat et proinde sanclus habelur. - Characletibus alalis belle distunclus. Lnngiludo $\delta$ poll. Cólor aigra cærulescente- et viridescente-nitens.

G. 35. HYDRICHLA**), Sv. Vattentrast.

-) Hab. gener fire pratedentis sed stocurabreviore; alis medioritius: remige primo fere nullo a ei 3 longissimis. Nonu. guner. Dii celeb. Temminck Pusior nobis minus idoneum videlur, unde hoc genuinnm gracum e voce $1 \%$ i.lj, hujus Nationis adoptavimus.

-) Hab. gener fere sturni, sed rostro Turdis ap. propinquans: copice ininore corporeque cressiure. alis caudsorue breviorilus Ala mediocres: reinige prino lirexissumo, tertion et dongissimis. Nomen Cincli sperici jumprilem ad genus Truga pertineuti impositum, ut genericum ineptum censuimus, wnde unitum necessum fuit, quol e vo-

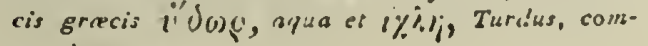
possimtes. 

1. cinclus: nigricanss collo autice pectoreque albis.

$f$. capile colloque supra fusccscentibus, collo antice pectoreque sordide albis.

Linn. S. N. 290. 5. Fn. 214. Retz. Fn. 200. Hamm. Fu. 227. Sturnus. - Nilss. Orn. 120 . Fn. 24z 1 Cinclus aqualicus. Poneopp N. H, 2. 117 . S2: Zool.

Sv. egentlig Vatlentrast, Vattenstare, Strönstare. - N. Quximkold, Forsekold, Jilvehonge, Strömstixr, Biekkefingl.

Hak. ad rimulos, flavios cararaclasifue non infrequens, solilarius. An Anvios inter lapides nidnm e gramisuibus muscisque consirnil, ord $4-6$ alba primo vere ponens et s 6 dies incubans. In aqua ad caput usque vallit el emergil cibum, pracipu insecla squalica, Inrvas enrum felusque piscium sibi quareus. Sonat inierdnni zerrk zerck! - Lunglludo $7 \frac{1}{6}$ poll. Loslrum nigrum. Iris pallıde-lusca, palpebire albre. Peiles anlice pallide-grisei, postice el subtus n'mro-fusca: ungubas luscis, celero o diagnosi bene cognila

\section{Nar. IT. A UPELIDIDES,}

\section{G. 36. MUSCICAPA *), Sv. Flugsnapp.}

1. grisola: fusco-grivea, subtus albida, capite collo inferiore pectoreque striis longitudinalibus griseo-fuseis.

f. minor: pedibus pallidioribus.

Linn. S. N. 328. 20. - 350. 10. (Diagnos,) Fn. 258. Mo-

-) Hab. gener. Corpus oblongo-ovale: cupise parun, collo brevi; alts retrogestis: reinige prino brevissimo, 5:0 longissuno. Pedes incalinares: tibiis longitudine digiti intermedii cum ungue ejus. Nomen gener. Brissonii ef Linate verbis lotinis Musca-cepio. tacilla Ficedula. Retz. Fn. 23z. Nilss. Orn, 90. Fu. 16is. $x$ - Sv. Zool.

Su. מu Flugsuap.

Hudp. Tracinue in houris cultisque nan infrequens; migral nodin Surl. at reverlitur menue Mojo. Vidulicat in sylvis ad irunruin arboris inter ramulos, ibique ova 5 caris'escenitoalba maculis rufescent bus pnit. Insecris presertim tiymanopleris sub volilu caplis victilal. ivon cantillal, sed pipilal st, st! et territus clamal $t s \mathrm{ch}$ th -reck,keck, keck!' - Longiludo $6 \frac{2}{3}$ poll heurum ngrum; basi xubius pallidum: anzulis a vis litesconnbus. Iris obscule-fusca. Rem:ges el iectirus ubscure-fusci, seculodarii cum lesiricilus majorlus margene exterinre

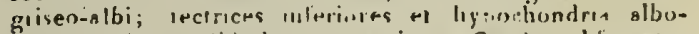

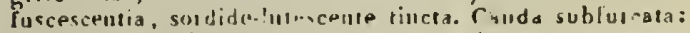
rectricibus fuscis, extima inagis griseo-lusca mergine exleriore basi pallide-grisea.

2. acricnpilla: nigra: fronte, macula ala. rum, rectricibus 2 latcralibus extus corporeque subitus albis.

f. fusca: maculis frontis et alarum minori. bus cte and diuribus.

Linn. S N. 3 io ro. (D-ser) Motacilla Ficedula 5.6. 9. Fn. ed. 1. 129. 1. I f. 229. m. ed. 2. 256. m. Metz. Fin. 2jo. Nilis Oriegr. Fn 162. 2. - Sv. Zool. Sv. Svarthäts Flusua P P.

-Junior: grisen-fusca: lateribus capitis fuscescentibus lutco immixtn, alis nigricantibus, fiscia angusti luten-albida.

Linn. Fn. fig. cis $f$.

for. 3. similis var $x$, sed maculis frontalibur bivis aibis.

Nilss. Orn. I. c. vir.

Far. $\gamma$. allat: capite colloque superiose silphurascentilus.

Spurrme. Mus. 74. 
Hab. in sylvis minus frequeus, D. Leche; var. ? tamen rarior in Goulandia, ipse, et var. y rarissima id Hollaian, D. Billing Nedio Sepl. migrans ad fisem Aprilis revertilur. Nidificat in cuvis vel inler radices arborum, ibi ova 6 cacrulescente-alba immaculara poneus. Pellicit: $k r i$ ! et liostea concrepat, zimzilulat lamen una insuave. - Longitudo $5 \frac{1}{9}$ noll. Rostrum arrum. Iris fusca. Cervix et Uronygiun nigra, fuscn-mixta. Remiges obscure-fusci: 5-15 basi albi, 16-28 albi latere interiore versus apicen nigri, lectricibus minoribus nigro lincis, e proximis: intmis apice albis unde macula aluri inarina. Rectrices nigta: extimis 2 latere exteriore a basi fere ad apicem albis.

\section{G. 37. AMPELIS *), Sv. Vinfågel.}

I. earrulus: rubrescenti.grisca: abdomine pallidiore, crisso fuscercente, vertice eristato, remigibus secundaris appendicibus membianaeesis.

f. macula gulari tugra: appicibusqus: Alavibus minoribus, appendicubus ad stumumu quinque.

Linn. S. N 297. 1. Fn. 82 Lanills. - Petz Fo. 208 Nilss. Orn. 89. Fn 155 - Sv. Zool 32. -

Sv. pladder Viufingel, Sadensvans, Rünnbärliane, T'nlfisparf.

Var 2. fulisionsa allat: capite obsembiome, rostro alloo versis apicesu ningo, fiente crissoguc fusco-rulis ternigibus restricibusque al-

-) Hab genor. medius inter Turd'un et Muscicapaan. Alcre l"nö",scular: ranipilus l et olongissimis,

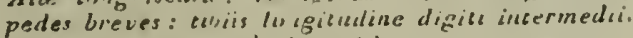

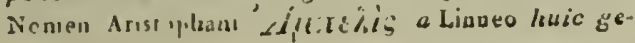
ners bribub.tm.

$$
19408
$$

bis apice lu!cis, appendicibustue alamun coccisteis.

Thunb. in Acl. Holm. 1808 137. 1. 4 - Nilss. Orn. var. $\alpha$. Sv. Zuol.

Var $\gamma$. alloa sublus vero doror medio re. ctricibusefuc alarum fücescenti ucbulusa; gula loraque uigris, rectricibus interurdiis albis appice luteis.

Nilss. Orı. - var. B. iv. Zonl.

Hab. "ibigne sat frequens, varietates lamen rarissimse in Uplandia $\beta$ (D. Lundéu), y (D. Paynulli. Nidulatur in regrumibus septentrionl puxinis. Insecta et baccie diversie, plinsertin bubl, victus ejus, Cantilia: injicunde tele: ziztri et peilicit: rhiss, thass! - Bostriln, fascia a rustro per oculos ad occipul, silla, rentigros 7 16 fascia crudie pedes'fle nigia; siria sule nindibi, inferiori alba. Homises firinotes $2-4$ latele exterus it a

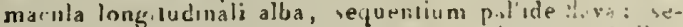

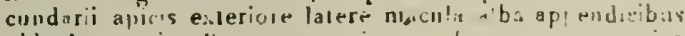

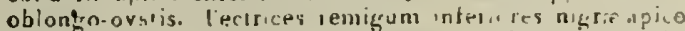

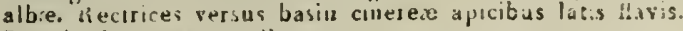
Longiludu circa 8 poll.

Coh. 3. Fissinoitses.

N'at. 12. IIILUNDINIDFS,

G. 39. CAPRIMULGUS ${ }^{*}$ ), $S_{i}$. Getsug.

-) cauda rotundata:

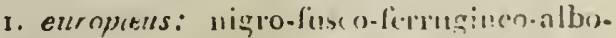
quc-varicgalus; sublus rubrescente-aibus fu-

-) Hab. gener. Ifirundinis Caprimu'gus appraximat:

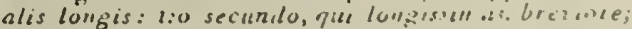
pedibus brevilus: digibs antirds articuls frims

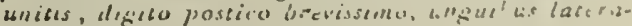

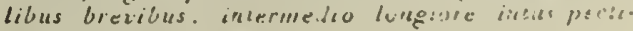

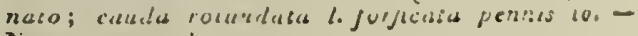
Nionsen gremer. lo:shumb. 

sconundulatus; cauda cinerca, transversim fit.

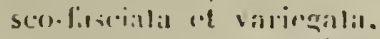

f: colure clarior: alosque inarula maris alha

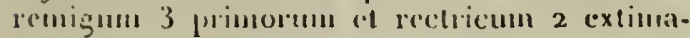
rilil.

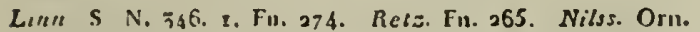
173. Fu. 402. - Sv Zool.

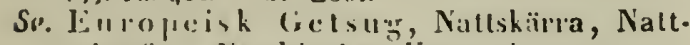
shraria, Nallblacka, Kärrylla.

Ual, in sylois haud passimi nugurans mense Sepr. re-

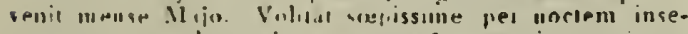

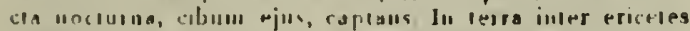

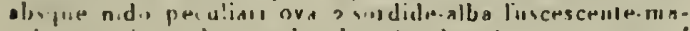
culala ronic w dias 14 incubat. Shidel: irrerp urrer!

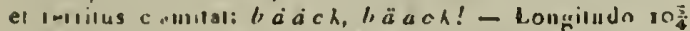
pull. liosfrum nighum. Uculi magui: iide carruleo-alıa. Cagut al disum cineres undulis punctisyue tenussiuss

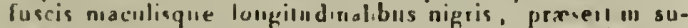
fla capul: ncipito siapulisque maculs pallide-[orlufi" neis. linea a unaxila inlenine ad lalera coll, decurreute feumginescente-abab. Collum inloune el pectus auterius. undulis Innussimas cinropis el nigricanibus, maculs in nimlio colli lestugineo-luteis. Abjumen cinereolmagi-

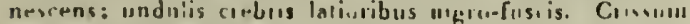

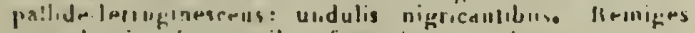

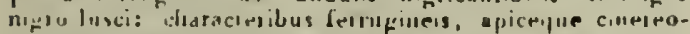

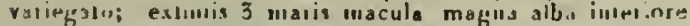

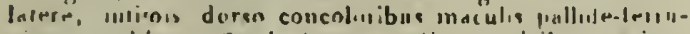

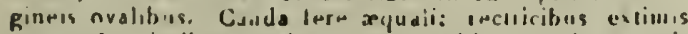
palum borvionitus, apice III mate albis. Iascis 8-9 ni-

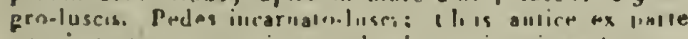

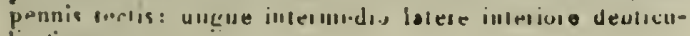
lis 6 jeclinisio.

\section{G. 3g, CYPSELLUS *), Sr. Mursvala.}

1. upure:

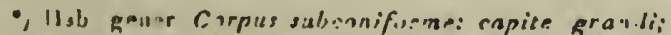

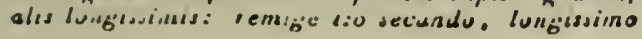

1. Apus: nigriians: gula alba.

f. lisco-ater: : suld sorrlide-albis.

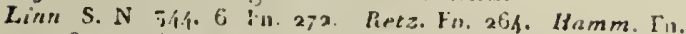

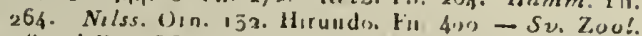
Sw. fistlie Milruvala, Tornsvila, Ring. svala. - N. Kingsvalc.

Hab. fere ubique, sed nimus foequn us in rimis murorum alforun et rudimorum, numiluam in alburibus, et iti

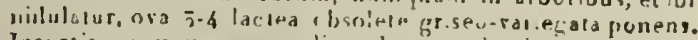

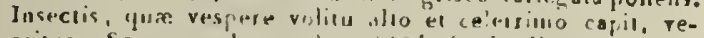
scitur. Snit., sun linertajo tiid! bacle dignoscilur. -

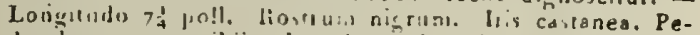

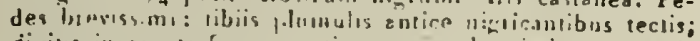
digitus incarnalo-fuscis, tulimo antuco bievissimo; unguibus acutissunis $11 \cdot g$ is

$$
\begin{aligned}
& \text { G. tu. HIRUNDO *), Sr. Svala. } \\
& \text { e) isuda forficala. }
\end{aligned}
$$

t) digitis all ungues usipue plumosis.

1. urbaca: nigut, sulutus nivar: mopygio tectricilnogue suprovoribus candx niveis; re. ctricibus iusuacerlatis.

longior, paulo breviore; pedibus brevissimis: digitis onnibus 4 antices ud hasin usque divisis unguibus brevlius crassiusculis. - N. fen. e gisca roce hililíi.us.

-) 1lab. grillo jerr prisederbis. sed alis minus bongis: remige $1: 0$ lonfessimo: pediluus brevibus, if:gitis; anticis et uno postiro graciliuribus, digilo extirbu ancico el inedio ad artirulum proinuin aduexu; caluda rotundato l. forficaba: pennis ts. N. jen. latthuin est. 
f. gula sordide altha.

Linf. S. N. 341. 3. Fn 278. Lietz. Fn. 262. HIzmm lin 262. Nilur. Oril 130 Fil. 39-2. - sc. Zowl

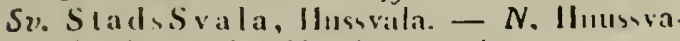
lc, Byesvale, Taish icesurale.

Ilab. ubi.jue freglienlissima. obrenil circa medum Ma jum, signum nareunn gelu desillentis, ahirus ad fiurm niensis Allg. Ni u'alur ut sengens m ove k-G alba punctis fus is nonil, ea dies 13 incubans Peliscil färr el stru. lefs! cantillans contulus nou iujucunde. - I.nngilldo

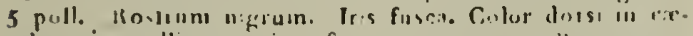

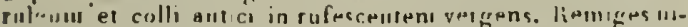

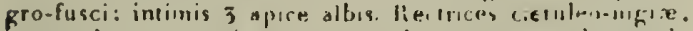

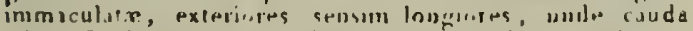
minus furficala. Pedes aibu-landli: ungrabus griseiso

tf d givis nudic.

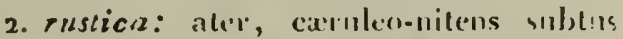
albida: froute gulaigue canlanceis; rectvicibus nigris, interenediis exe'plis, intus macula allsa.

$f$. cauda minus forficata: recticibus cxti. mis minus elongatis.

Linn S. N 345. I Fin 27o. Retz. Fin. 26r. Hlamm. Fit 261. Nilss. IIIn. 120. Fin 595. 1. - Sv Zuol.

S21. LandSvala, Ladusiala. - N. Late. svale, Marksvale.

Var. B. tota alba.

Nilss I. c. Sv Zool.

Hab ad dumos vilgalissima, uhi sub sectis nidum seminhb isum apeltura laler'sli aediticat pt ova 4-6 a ba puucis rufescenubus violaceisque bis per annumi fioml. Ex Urnishamy Hirundinis in uidn persecjullur. Jilseila ravia, p xeserliun culices in acie et sujirio superfictem aquxe, cibus ejus. Clamilat sope zifot! el candil tere exprimit secundum zermanos: da ich fortzog wa- en allen Kisten und Kasten voll, da ich wioderkam,datch wiederkam,war alles wise is nd beerer! - Lollgiludo Gl-7 poll. Kosirum nigrum,

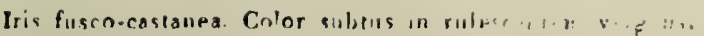

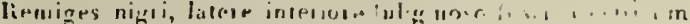

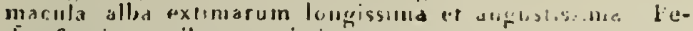
des fusci ungruiluss acutissimis

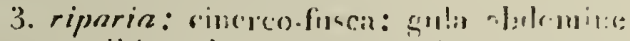

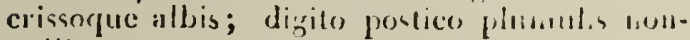
nullis.

Linn. S. N. 344. 4. Fn, 27\%. Retz. Fn 267. Riles Ota

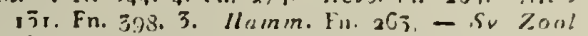

Sv. Strand,vala, liaknualil, Jordsialia. N. Siranduale, Saludlöinter.

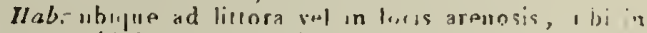

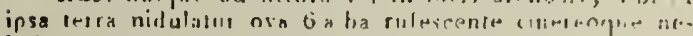

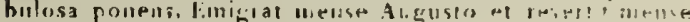

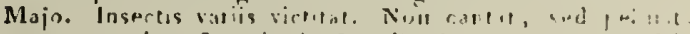

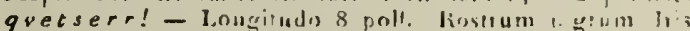

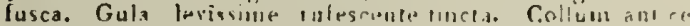

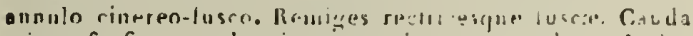

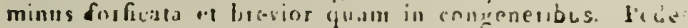
fusco-nigri, nudi, dugreo pussice excepto.

$$
\text { Coh. 4. Trsurnosarers. }
$$

Nac. i'. Cllillir z!i)ES, G. 41. SITTA *), Su. Nitvaita.

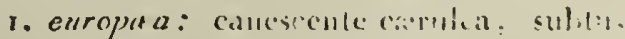

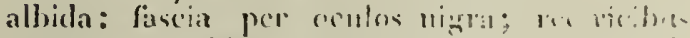

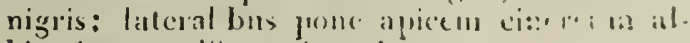
bis, intermedis 2 citictedis.

- Hab. gen. fire ul in Picis, sed cispire muinore. a. s.

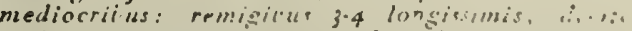

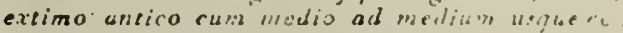
nexo, postico longissem!o ung..e lo"? ct i" rvist.

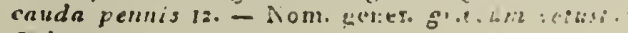
sirti: 
f. calore liallidione.

Linn. S N. 1.?. 1.13. ro4. Retz. Fn. 5g. Nilss. Oru, 促. Hotnim. Fn. p. 2G. - Sv. Znol.

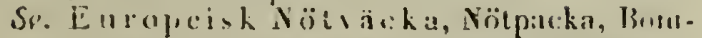

klfiar - N. Nalvahle, Egde, Spueturise.

Ilab. in sy'vis frondocis silt fretiluens, manens. Ve-

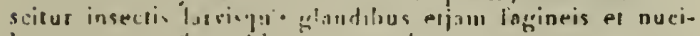

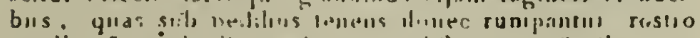

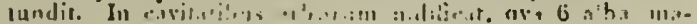

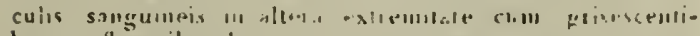

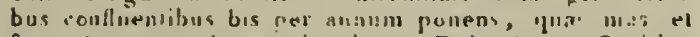

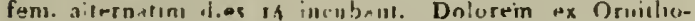
mya aviculans valdo pasill, peilicil deck, deck, deck et clalld hy der dyr ifre dis r! - L.nngilldo 5 ! poll. Rosirum cexrilescente-fuscunt, sublits albidum Jris zriseu-finsca Fiuns cermlea. Coinr sublus pecturis abciomivisifum in rulescells reigil. Hypochondria rufa. l'edes grisescente lulei.

\section{G. 42. CERTHIA *), Su. Kröla.}

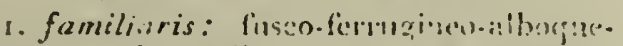

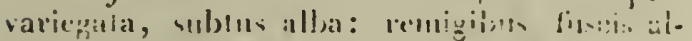

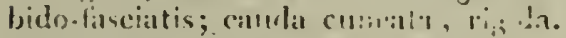

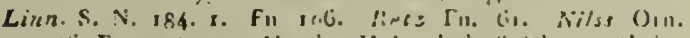
45. Fn 3.4. 1. - Norske Videllskab. Se'skellyshririer a E. nag. 36 ? c lig. Mulacilla seolopinciins.

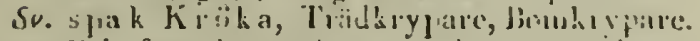

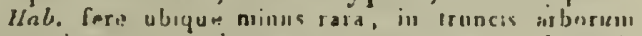
currens, insecin, ura larvasijue enrilm quarens. In civis

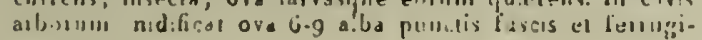

-, Hsb. gener. fere anceralentis sed rorpore cras-

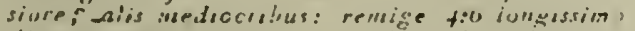

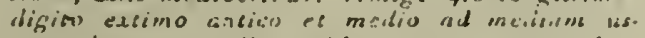

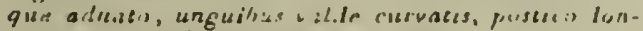

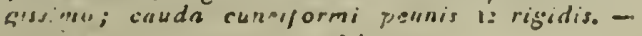

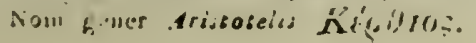

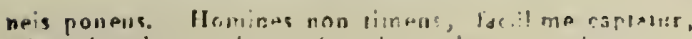

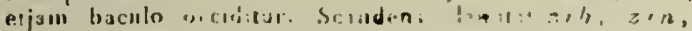

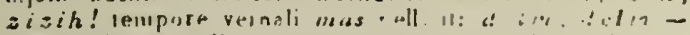

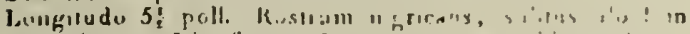

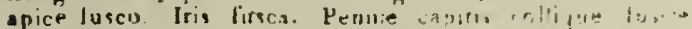
unacula orali s'bur lascis per oculus fisce al erom

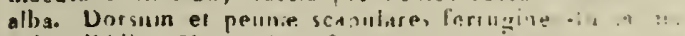

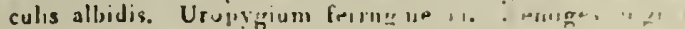

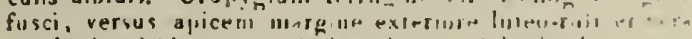
sus basin viriderseute-gıi, mi: pimus of bisin 1, ....

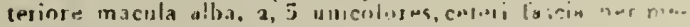
dium alb. Cauda decurvala, eximess s.alleifn! in:. c ":1posits in duas cespulas divisa: prent ibns 12 gresaj inscis, extinois illacua alba. Peiles pellide-lascl: ungribus griseu-albis, postesinte plizionán.

$$
\begin{gathered}
\text { G. } 43 \text {. UPUPA *, Sv. Härfågel. } \\
\text { •) nat b:s nudis. } \\
\text { t custate: }
\end{gathered}
$$

1: epops: nigro-iliboquce varia: crista a. pice nistis, capple collo pectureque ferrugineo-luleis; abrlounine albo; cauda nigra albo-fitcinit.

Lirn S. N. 185. 8. Fn. 105. Rietz. Fn. Go. Vilss. Orn.

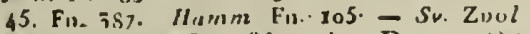

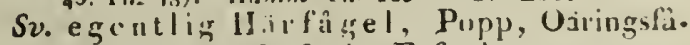
gel. - N. Herfuyl, Erfuzl.

Hal. in sylvis sal rara; migrans. Inseclis precipue coleopleris vichllal. Niditicat in cavis arboruin ova 4-6 sordide cinerascentia ponells. Temprore rernali et au-

- Hab gener. collo longicre quam in procedentslus; alis mediocrubus: remigalus $4 \cdot 5$ iongissimis: pedilus mediocribus, digitu extimo et ine.lio arr tiiulo 1:0 unito, unguibus brevilus et pariser rurvatis, postico subrecso. Calida suhquisu'sits pennis 10. - N.gener. latınum Geszeri est 

Iumntli crial hupp hupp hupp? - jo jo to to!

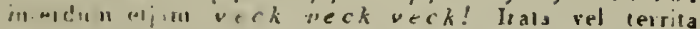

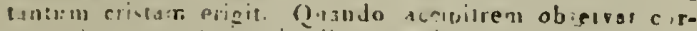
pus al torlum defiessil alis extensis, cap:le ad Jursun

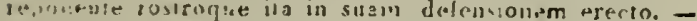

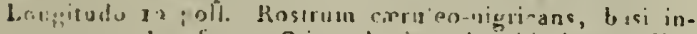

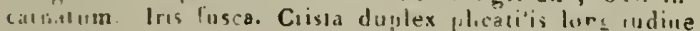

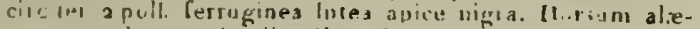
que complicate, Isciis albis nigra. Piales cinerei.

\section{Legio 5. HOLOSYND ICTYLI,} Nat. rq. LEROMIDES,

G. 4千. MEFOPS *), Sv. Bihn̈k.

- tectricibus intermediis longiosibus:

1. apiaster: capite obituc castanco. finn-

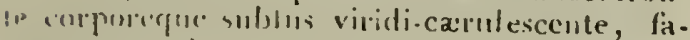

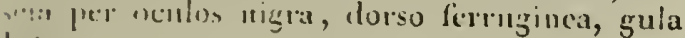
Ji1!c i.

f. rubre pallicline, forcia Jutescente supra

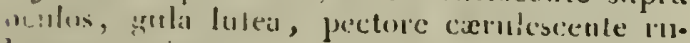

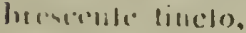

Lim. S. X. 1S2. 1. Nilss Otn. 2. suppl. $259-$ S.. Zoul.

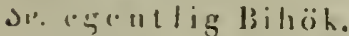

./un. "1й viridescente. brunneus: fascia sı-

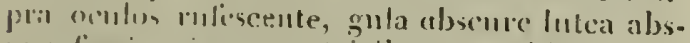

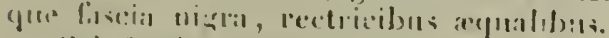

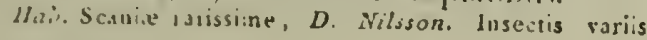

\footnotetext{
- ildi. pan. fere Carthir: a!is mediocriluar, remige

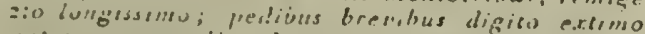

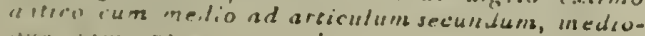

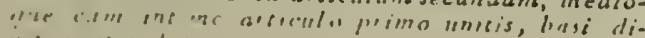

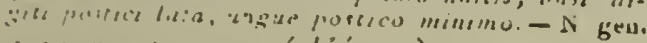

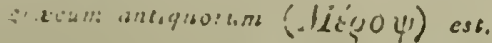

viclitat sub volatu hymenoptera captai, sed illa sedens edit, ividificat, forsan in Scania tanlum, more Hisundiais

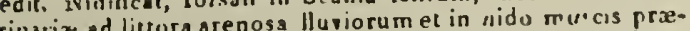
riparia ditura alenosanil. Sibilat, sisikryi! - Lon-

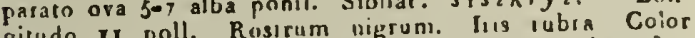
gitudo II poll. Rosirum lagrun. Ils Iubra Coior Jorsi inlerioris in lusescentem vergens. Medium ala rulescens: remigibus et rectricibus nlivaceo-vir dibus. Gulalutea cunı lascia nigra termunuta. Pedes brunnei.

\section{Trib. 3. ANCYLOKHAMPHAE,} Nat. 15. ACCIPITRIDES, Div. x. canile rel collo impenni:

\section{G. 45. CATH IRTES *), Sv. Rensgam.}

I, percnuplerus: albus: remigibus primoribus nigivis, cute capitis collique inferioris lutescente livida; rostro nigra, cera aurantiaca.

f. inta fusca.

Linn. S. N. 1237 Vuliur. - Euffon (Planches enluminees) 420 . Vautour de Norréne. Sv. Zool.

S\%. svartvingaRensgam.

Jun. obscure fuscus maculis sparsis lute. scente.brunncis: ente capitis collique inferio. ris livida, plunulis raris grisescentibus; cera rostri jedibusque cincreis, iride brunnea. Linn. S. N. ed Gmel. 1. 1. 248.8 Vullur fuscus, Buff (PI. enl.) 427. Vaulour de Malihe.

-) IJab. gen. Aq̧uile, sed alis leviter acuminatis reinige 3:0 longissimo; pestebus mediocribus, alis: nudis, digito medio longissimo $\mathrm{rum}$ extimo as lasin unito. - N. gener. e graco voce huJ́lín. $\tau, s$, purgabor, Hliger. 

Hab. Norregixe rarissime (D, Com. Buffor), ibi ta. men rix domicans. Cadareribus, immundilutibus variis rescilur, raro mammalis rel ares rivenlos apgrediens. Nidificat in rimis montium el locis inaccessibilibus. Sino omni dubio esi Vuliur percnoplerus antiquorum, el aucloritele plurium auclorum hunc ex numrro Avium Scandinariz excludere nobis haud concessum esse credimus. -Longitudo 2 ped 1.3 poll Itis lulea Remiges 2 extini toli nigri, celeri prinores margine exteriore canescentes. Pedes audi lutesconte-lividi.

Div. 2. capile plumoso, Subdiv. I. rosiro edentulo, Manipula I. ad basin reclo:

G. 46. AQUILA *), Sv. Örn.

-) libris tolis plumosis:

1. Chrysuëtus: finsca, ferruginco immix. to: capite colloque fulvo-femmineis, sublus nigricantibus; cauda basi alloa vel cinereo fuscoque variegata, apiceque nigricantc.

f. mari similis sed imain.

Linn. S. N. 125. 5. Fn 54. Kesz Fn. 1. Nilss. Orn. I. Fn, 40. x. Hantm Fn. 68 Falco. - Waihinceister in dct. Holra $180 x$. p. $18 \%$. Falco fulrus $(f)-.S v$. Zool.

Sv. Guldörn, Landtörn, Skogsöın, Slagörı. - N. Landörn, Fjeldörn, Slagü̈rn, Kongë̈rn, Skiorvenge 1. Skiorvinge. Var. 3 . dorso nigricante: cauda cincreo

-) Hab. gan. corpus aqualiter crassum: rostro robusso; alis longis: remige 4-5 longissimis; pedibus validis nervosis, bibiis nullis l. plumosis digiis rohustis unguibus acubis valde curvatis. - N. gen. labinum. parum variegata ab apice ad tertiam partem niwra, subtus fasciis $2 \cdot 3$ angustis cinereo-a!bis. Nilss Orn. 1. c. Sv. Zool.

Var. $\gamma$. dorvo fusce pennis nigricantiuus immixtis; capile colloque ferrugineis; cilluda alba I. sordide alba, $\frac{1}{2}$ ab apice nigricante.

Linn. S. N. ed. Gmel. 259. 54 . Falco niger Reez. Fu. 2. Falco melanaëlus. Nilss. l. c. juniur. Sv. Zool.

Yar. D. dorso fusco, catuda alba, lateribus basis apiceque nigra.

Linn. S. N. 125. 6 Falco fulvus. Rets $\mathrm{Fn}$. 2. Falco na lanac̈lus 3 Parelius in Act. nilios. 4. $421.54 . Z$ Var. $\varepsilon$. lotus ex albo flavescerus.

Linn. S. N. ed Gmel. 457. 47. Rebz. Fn. bo. 2. E. 11. lanaetus $\gamma$. Sv. Zuol.

$H a b$, in tola Scandinaria, sed minus freonelly, sw E lamen in Scania tarissima, D. Retziss, pronsul

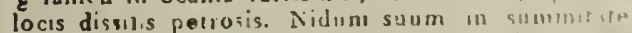
rum excelsorum $\mathrm{vel}$ in monibus alu: indiarai sordide-alba ponens. Leporibus et inamualibus 1. . avibusigne proprie vescitur, aggredunt vers th olos carsindusque, simul ac nou sine exeni? cadarera diversa stqui boniquo fucie. - 1 3 f. $3 \frac{1}{2}$ ped. Rosiruin caerulea-isigrionas cera iridique plus minusve lutess. Peista wh longse, acuix, splendentes fulvu.leris.. mixio, penuis fronlis obscusiores, I'r oculos albida setis nigris adspersis $G_{1}$ scura fuscre l. nigricalles. Dinsum el lectrices alarun lusca, nigho tenn mixlis. Caissum et femora iii ws r te rufo-fusca Remiges nigricantes. "? cinerascontibus albnevaluega is, a acente. Tibice inbustio, arie ce l albo-plumosx; dignas lusers,
2. nevis: rufofiun 1): ।

ribus nigricantibus basl allis 

sclure-fuscis, lineis transversis cinerascentibus, apicibilsque albidis.

jun. colore obscuriore; tectricibus alarum, peunis secimdariis rectricibusque subtus maculis apicalibus ovalibus grisescente-albis. .

Linn. S. N. ed. Gmel. 258. 49 Werhem. in act. Holm. sko 1. 187. 5. Nilss. Ora. 2. Fn. 45. xa. Folcu. $-S v$. Zunt.

Si. Iy

Hab. in Lapponie (D. Paykull). Historie ejus ignola; cagnorcirur rero, quod in orburbus Helvelize nidifical: vix in Scandinavia, ubi adultiores, es biennes lantum obrenieruns. - Lougilodo m.22 poll., fern. 2 ped, Rosrum cartuleu-nigricans: cera lutea. Kegin inter uares -l oculos aetulis nigria adopersa. Caput el femora colore cupporis, and dilutiora. Criasam albo-rufescens. Remiges secundarii nigncanlea basi cinerasceinies, apice dilu. Pinres: leciricibus alaruin minoribas pallide rufo:fuscio margivibus adhuc pallidioribus: lectricibus inferioribus rufuofuscie meculis elbig. Caude elis narum lnogior: 1eellicibus infor. albis. Tibiz antice cinereo-rofo- postice albo-plumoses digitio luleis.

-) tibiio non totis plamosis:

3. Inuoocophaln: nbscure biunnea: capite, collos superiore, tectricibus enuda rectricibus. que albis; rostro, cera pedibusque lutescenle. nllbir: iridinge albida.

Sv. Zool

dw. It villi ufd bru.

bibnnis: irregulariter ferrugineo.fuscnque. macululu; rostru nigro: crera lutea; cauda aliv Inngune: res:tricibus nigricantibus latere inleloures alloidist pedibus lutescentibus.

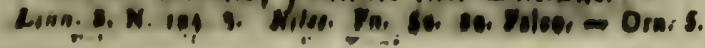

jun. pennis capitis collique grisescente-brun. neo immixto.

Hab. passim in Lapponie, D Paykull. Pisces virenles capit ad cibum summ; celero oconomia ejus adluc ignuta. - Longitudo fem. $2 \hat{z}-3$ ped; in. minor. Color corporis brunneus cum elate infuscalur, sed idem rourti, cerz, iridis et pedum dilıitur. Regio inter nares et oculos seris nigris adspersa.

4. albicilla: fusca griseo imınixto: capite colloque sordide albis; uropygio caudaque brevi rotundata albis.

f. mare paulo major: colore cápitis colli. que pallidiore.

Linn. S. N. 123 8. Vuliur - Fn 3. Retz. Fn 3. Wochtm. in act. Holm. 1801.175 . Nilss. Orn. 5. Fa. 47.12, Hamm. Fn 6g. Sv. Y.00l. 8. Falco. -

Sv. Hivitstje rtsörn, Fiskörn, Hafsörn. N. Fiskeöı'n, Mundgotsfugl.

jun'. cera flava, corpore ferrugineo-nigricante striis flavis; reclricibus intus albis.

Linn. S. N. 124. 2. Falco mel inaélus. Hamm. Fn. 78. Falco osgifrogus. Sv. Zuol 124 4.

Hab. in locis nemorosis el rupestribus pracipue juxea maro el lacus ubiqua sal frequeas. In aboribus excelgis el montium declivibue ad mare nid,ficans ora $\$ .5$ rotundiuscula cordido olbe sparse rubreacente-maculase noair. Caderere veria, mammalis minoria, ares el picer cibum ei prebeni. Adlive jurenis facillime cicurolur. - Longitudo fom. 5 ped in. paulo minor. Rostrum cerinumi core lutpa llegio inier narea ef oculoe

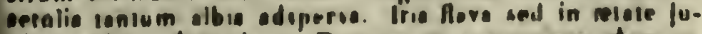
hinre colore obecuriere. Doreum al uropyesum obscire-

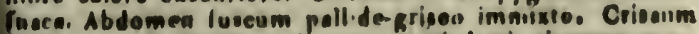

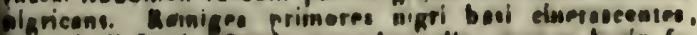

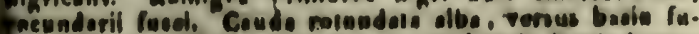

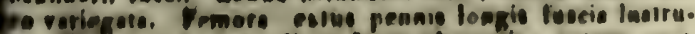

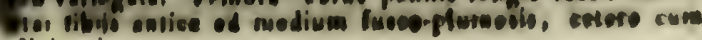





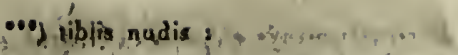

5. Holinëtus: cápite collo corporeque sub. tus alkis; laleribuis colti dorsoque fuscis; cesa pedibusgatue cæruleis"; "femnribus brevissime albo-plumosis, unguibus subxqualibins tereti. bus.

-f. major: capite súpra pectoreque magis maculatis.

Linn. S. N. ag. 26. Pn. 63. 'Retz. Pa. 5." Nitss. Orn 6. Fu 64. 15. Homm. Pu: 74. Palco. Svi Zool.

Sv. Hafsürn, Sjöhök, Fiskliök, Fisktjuf, Fiskkjuse, Blâfot. - N. Blaafot, Fiske.

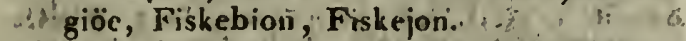
- jun. capite supra peclorcque magis macu. latis, dorso alisque fuscis, marginibus penna. rum albidis; crisso yufescente; fasciis gaudz evidentioribus.

Var. B. similis rar. á sed pectore anteriore fuisco maiginibus pennaxum albicantibus, Nilss. I. c.

* Hab. iper lolom Scandinaviam. ad aqus, aon infre. guens ubi gisces tirias ad vicium predalur. In arboribus allis L rupibus prope aquam dolcem nidulatur ;i ova 3-4 Iurescente-elbe maculis magnie puncrulisque sparsis rabrescecitibus ponons 1: Clamiat krauh! 1 rauh! heih, kei,h. Facillime in capturam piscium mansue4titar. - Longitudo m. 22 poll. fem., ped. Rostram nigricante-cerúleum. His pallide, hlava. Regio inter na. res et aculos setis aigris edspersa.s. Capus ef pectus, siriis maculjaque fuscis apersiss, macoh, oblongn magna fusca ab oculis ad latera calli. Remigei ubscure fusci, basi et ad partem lasere inseriore albi transversim fuscó-fosciali Canda alis, bravior. xequalis obscurer cinereo-fuscoques fasciara abjus albida: lasciis fuscescentibus; rectricibus

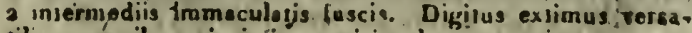
pilis, unguibus nigris in sevicirculum curratien $t$,
6. lércopsis *): testacca maculis nigro. fuscis, subtus cum fronte albida striis macu. lisque fuscis rostro nigro, cera cærulescente. ff minus - albida, maculis numerosis et ap. pioximatis fiscis.

fechstein Naturgesch. Deuilschi. T. 2. p. 5i2. Sy. Zool. Si. livitsyns Örn.

inn. colore superino obscuriore, subtus pallide, testaceo maculis ferrugincis numerosis et approximatin.

Linn S. Ne od. Gnel. 259,52 , Falco gallicus. Nilss Ora.

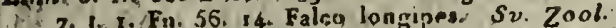

- Hh Hab. in sylvis Lapponize septeptrionolis rarissima, D. Paykull Lacertis sespentibusgue imprimis, raro avio bos victiter. In -orburibus allis nidificans ara $2-\overline{3}$ griseceonti-irsmaculats ponit. - Longiludo 2 ped. Caput magnum a iride Jutea, regione circa oculos adali plamulis Gibidis. Remiges primores nigri, ceteri cum teciricibus. oplorn dorsali. 1: Cauda subqualrala grisescense-brunuea: lisois srausversis : fustis, sublus albida. Tibize pralongx: th digiti grjescente-crerulei.

- w. G. 46. MILVUS **). Sr. Gladz. "

41 fegalis: furugineus: "maculis fuseis; capile colloque albicantibus fusco-striatis; ce-: a pedibusque brevibus lutcis.

f. colore nallidione.

Linn.'s. N. 126. 12. Fn. 57. Reiz. Fn. 1r. Nilss. Orn.

-) Antiquirsinum romen specificum a D. Comelin datum inepusm censuimus, nec nominam numerum ougere voluiunus, adeoque quam dedit D. Bechsiain detiominationtem rétinuimus.

$\because$ Hipb. gener. fero,praceddencis sed alis langis : re. mige 4:0. longissinas cibitis brevibus poulo subge. nu plamosis. - N . genesr. letinum Gesneri-esc. 
10. Fn. 60. 15. Hamm. Fu, 73. Falco Milvns. Sv. Zool.

Sv. KongsGla da, Glänta. - N. Glente. jun. magis ferrugineus: medio peunarum niguicaute; pennis capitis brevioribus, rotundioribus, absque striis longitudinalibus; maculis supra collum inferiorem albidis.

Linn. S. N. ed. Gmel. 262. 6.3. Falco austriacus.

Hab. ubique sal vulgaris. Emigrul circiter mense Septembri, redux ad finem Martii. E volatu ejus natante altiore serenain et depressiore pluviosam tempesiatem volgo prasagit. Animalibus minutis, aribus piscibusque cadaveribus et helmınticis variis vescitur. Nidifical in arboribus, ora 3 sordide-alba maculis obsoletis rufescentibus ponens. Clamilat: $k y k k y \bar{a} h$ ! - Longitudo 2 ped. 2 poll. Rostrum nigricans: cera irideque llava. Regio inser nores et oculos, menlum et osbita setulis nigris. Iris lutea. Dorsum et alze pennis obscure fuscis marginibus lale ferrogineis vel albirantibus, Sublus ferrugineus stris longitudinalibns fuscis. Crissum pallide ferrugineum. Remiges primores nigri, larere incriore a basi ad medium albi, intermedii 6-8 extus ferruginei intus albi fascii, fuscis; secundarii nigricantes apice albicantes lalere interiore pallidinres obscure-fasciatio Cauda forkcala ferruginea. Tibiz breves antice ad medium luro. scente-albo plumosis, celero cum digitis lutex: unguibus acurissirais nigris.

2. ater " ": grisescente-obscure-, subtus rubrescente-brunneus: maculis oblongis nigricanlibus; capite colloqne albicantilous striis bruuneis; cera pedibusque aurantiacis.

-) Speciman hujus avis, quod in Mfuseo Grilliano $t$. nunc semporis D:i Tham servalur keste D:o Lindroth. de Ornibhologia maxima jide digno, in Lapponia superiore occisum est, quam ob caussam e Fauna rosira hanc avem excludere non fos sit.
Linn. S. N. ed. Gmel. 262. 62: Nilss. Fn. 63. Falco. Orn. 1o. Falco Milves junior. Su. Zool.

Sv. svart Glada.

jun. colore obscuriore brunneo l. nigricante, peunis capitis nuisus acutis, corporis rubrc. scente-marginatis; colore ceræ pedumque miuns ardeute.

Lınn. S. N. ed. Ginel. $26 x$. 6r. Falco rgyprius.

Hab. in Lapponia hyperborea rarissimus, D. Grill. Animalibus diversis minulis presertim piscibus victitat. Nidulans in arboribus, ova 3-5 lulescente-alba maculis numerosis et approximatis brunness ponit. - Longitudo 1 pell 10 poll Rosirum atlum eilentulum. Iris grisescente-nigra. Remiges nigricante-brunnei. Femora obscuse rubrescentia. Cauda porum forficata grisescentebrunnea: lineis irangversalibus $9-10$ clare brunntis.

\section{G. 43. ACCIPITER *), Sv. Hök.}

I: polumbarius **): fuscus, subtus al. bidus: undulis numerosissimis fuscis; cauda longa rotundata: fasciis latis 5 fuscis cinereisque.

Linn. S. N. 130. 10. Fn. 67. Retz. Fn. 22. Wachlm. in act. Holm. i8n2. 249. Nilss. Orn. 13. Fn. 34.7. Hamm. Fn. 78. Falco. - Sv. Zcol.

Sv. DufHök, Slaghök. - N. Storhög, Due. falk.

-) Habit. gener. Milvi; sed capile proportionaliter paulo minore; alis brevibus, vix duce tertice partes cauide longis, remige 4:a longissima; tibiis digitisque longis, unguibus valde curvatus et ocutis.N. gen. Latinum vetustum.

-1) Quia haec species vulgo nominatur Hök, denominationem antiguem latinam Accipilris ddopravimus. 
Var. P. similis jumiori; sed pennis dorsi tectricunque alarum albo-marginatis.

Linn. S. א. ed. Gmel 266. 75, F. Gallinarius, var. nxvius. Nilss. I. c.

' $a r, \gamma$. albus maculis tantum longitudinalibus capite, collo tectricibusque alarum. linersque: it pectore abdomine femoribusque rinfescentibus.

Niliss. I. c.

jun. ulsscure-fuscus: lateribus capitis collique ferrugineo abis stris longitudinalibus fusicis; fucits cande albo-maryinatis 4.

Li:n. S. N. 20. 13. Fn 5s. Retz. Fu. 13. Falro gentilis. Linn. S. N. 4 d. Gntel, 265 73. W ach $6 m, 1$, c. $25 x$. Fisio fiallinamias.

Ilab. per lotam Senndinaviam sal frequens, manens. Vasiesates lamell rariores presertm y e Norrlandia, $D$. Pariull. Avum garvarum, speciation domesticarum es I hoslis frecissimus. Nidifical in arbore excelse el ibi ponil ora 4 lutrescentia nigricente.maculata et siriata. Sibilal: grigrih grth. et gihgia! Pro venatu estimatur juniris, - Longuludo jem. a pell. maris paulo niinor. Kontun nigrum basi cxerulescente, cera viridescenlm. Jusea. Lesta setulis uigris. Iris, anguli oris et margo palpebrarum fari. Caput supro obscure.fuscum: superciJia alba. Cervix. dorsuin alixque supia fusca. Subtus albıdus: limeis fulle longindinatibus- sed in jugulo, pectore, abdomine, lemoribus alisythe subtus iransversis undulatis luscis. Remiges lusci, obscuriore-farciati, latere iniariora basi maculis atbis. Cauda cineres: fo. scirs 5-6 lanis funcis, extina lanore, apice albido. Tibit antice sem:l..clin: viridescante-futese: digitis brevibus, un. guibus validis nigro-fuscis.

2. nusus: c:ierulenecincreus, subtus albus tratiservitrs derruginco-utedulatus: macula nu- chali alba; cauda longa æquali cxruleo cinerea fasciis 5 nigricantibus.

$f$. major, fusco-cinerea, subtus alba undulis fuscis.

Linn. S. N. 130. 31. Fn. 68. Retz. Fn. 25. Nilss. Orn. $14 \mathrm{Fn}$. 35. 8. Hamm. Fu. 76. Falcu. - S . Zool. Sv. S parfltök. - N. Spurhög, Spurehög. jun. obscure-fuscus: pennis ferrugineo-marginatis; fronte supercilisque albidis fuscostriatis; subtus ferruginescente-albus: striis juguli, maculis pectoris cordatis, fasciisque abdominis fuscis 1 . obscure-ferrugineis, fuscomarginatis.

Hab. in sylvis sat frequens. Hostis columbarum et aviun minorum, sed muribus, lacertis vermibusque etjam victitat. Nidificat in arboribus el frutetis, inlerdum etjam montibus prope canipos pratosque, ova 3-5 sordidealba maculis ferrugineis ponens, qux septimanss 3 incubat. Clamitat $g \ddot{a}, b \ddot{a}, g \ddot{a}$ et $g i c k g i \in k$ ! Pro venatu non ineplus arit. - Longitudo fem. $14, \mathrm{~m}$. 12 poll. Rostrum carulescens pice nicro, cera viridescente-lutea. Iris flava. Speciei antecedeniti similis, differt tamer prater characteres indicatos: hmeis gulae jugulique ferrugineis, lateribus juguli in ferrugineun vergenubus, pedibus, prasertim digitis proportionaliter longionibus et tenuioribus, digitis etjarn sublus nodulis verrucosis, unguibusque nigris.

\section{G. 49. BUTEO *), Sv. Vråk.}

") tibiis nudis:

1. variogalus: fuscus, subtus lutescente.

-) Hab. gener. Accinitris; sed rostro parvo; alis mediocribus: remige 4:0 longissimo; libiis brevibus. - Noun. Gener. Plınii latinum est. 

albus, fusco-maculatus: cauda brevi cincreofuscoque transversim lineata; tibiis antice plu. mosis.

Linn. S. N. 127. 15. Fa. 60. Retz. Fn. 16. Nilss. Orn. 11. Fn. 64 16. Hamm. Fn. 70. Falco Buteo Linn. S. N. ed. Gmel. 267. 78. Falco variegatus. Sv. Zool.

Sv. słãck V råk, Musvråk, Musvåg, Qvid. fâgel, $-N$. Skiörvenge, örnefalk.

$\nu$ ar. $\beta$. 'fuliginoso-fuscus: abdomine rarius albo-undulato; crisso albo fuscoque-fa. sciato; femoribus immaculatis.

Nilss. l, c. A. Sv. Zool.

Var. $\gamma$. similis antecedenti sed minor.

Nilss. l. c. G. Sv. Zool.

$V a r . d$. fuliginoso-fuscus: gula juguloque albidis fusco-maculatis; abdomine transversim albo.fuscoque-fasciato; crisso rufescentcalbo maculis rasis transversis fuscis.

Nilss. I. c. B. Su. Zool.

Vur. E. fuscus: pennis colli antcrioris ala. rumque tcctricum ferruginescente-albo marginatis, macula majore sub oculis oblonga albida; pectore, abdoinine, crisso ferrugine. scente-albis; uropygio lutescente-albo, medio macula magna fusca; cauda lineis transversis numerosis cinereis fuscisque basi adque raches ferruginescentc-alba.

Nilss. I. c. C. Sv. Zool.

$V$ ar. 2. capite colloque albis maculis ra. rioribus fuscis; dor'so alaruntque tectricibus fuscis pennis albo-fimbriatis; pectore, abdo. mine femoribusque extus albis, intus crisso. quc rufescente-albis; rectricibus mediis albis transversim fusco ceteris fusco.cinereoque-fa. sciatis. (ulter. ut in $\varepsilon$.) Nilss. l. c. D. Linn. S. N. ed. Gmel. 272. 89. Falco rer-
sicolor? Sv. Zool.

Vor. $\eta$. capite corporeque subtus albis; vertice maculis raris fuscis, seriatim ab an. gulis oris ad latera colli, confluentibus pectore superiore, nonnullis parvis abdomine, et in tectricibus alarum minoribus albis. (ce. cero ut in (.)

Nilss. l. c. E. Linn. S. N. ed. Gmel. 267. 79. Falco albidus? Sv. Zool.

Var. \&. capite corporeque subtus testaceo. albis maculis raris ad latera pectoris fuscis; dorso tectricibusque minoribus testaceo-albis fusco-maculatis; remigibus fuscis: sed quarto ceteribusque apice albis; rectricibus mediate interiore apiceque albis, exteriore cinereis tuansversim fusco.lineatis.

Nilss. 1. c. F. Sv. Zool.

jun. colore clarius brunneo albo et Jute. scente-vario, gulla albida striis biunnescentibus; pennis pectoris albu-marginatis; abdomine albido maculis magnis ovalibus et cordatis brunnescentibus.

Haly, sat frequens, variptates vero omnes rarioses: B. $\delta$. $\zeta$ in Uplandia, $\gamma$ in Lapponia, $\varepsilon$ in Norrlasdia, et $\beta$, ${ }^{2}$ in Scania, sub hieme migrans. Nidifeat in summis arborum, ova 3.4 ponens. Mammalia avesque millnra, ranxe, laceria, serpentes, animalcula aquarica, conchx \&c. cibum ei probent. Sibilat kriä, $\lambda r i \ddot{a} !$ et clamitat interdum roce ejulante $g a a h !$ - Longitudo ped. 8 poll. Hostrum nigricans: cers anguloque oris $\mathrm{Ha-}$ vis. Iris pro xtate varial fusco-grisea, rula el pallidegrisea. Sunra fuscus: marcinibus nenuazum rilus mi- 
nusve ferrugineis et albidis; subtus albus lutescente-tinctus niaculis fuscis pectore maj...bus lese cmoluentibus, sed abdomine distinrtis cordatis; guia fucco-suiala $t$ crisso maculis raris coldatis lasciali:; lemiges primures nigricantes latere interinie a basi a'bi, secundarii lusri a pice a!bicantes latert interinre alli fusco fasciati. Cauda subrogualis: recuricibus obscure cinereis lasciis ongustis unmernsis 10.12 fuscis, ipice et latere interiore ferrngineo-ali.s; fenora Jusca marginibus pennarum ferrugineis, cetero pedibus lufeis: unguibus nifris.

2. apizorus: filsills, silblis albus fusconaculatus: lora plumova, canda ciucreo.fusco. quc-fisciata; unguibus rectiusculis.

f. fuliginosofusca: fronte cinerco-cxrulea: collo antico maculis maguis brunnescentibus; pecture abdomineque lutescenterubris maculis obscure. brunneis.

Jinn. S. N. 1 ju. 28 Fn. 65. Retz. Fn. 7. Nilss. Orn, 12. Fn. 68. 17. Hamm. Fu. 77. Falco. - Sv. 7.ool.

sv. li i Vı ìk, Slaglı̈k, Vesphök. - N. Slag. l.iig, Spendh $\ddot{u}_{g}$, Moradshögg, Mleselıög Musevange.

I'ar. o. similis a sod macula nuchali alba. Retz. I. c. sv. Zool.

l'cr. $\gamma$. (m.) obscurc fusca, sublus alba: noculis distantibus rotumdato-cordatis fuscis, grula lirsco.striala, crissuque transversim fusco. maculatis; capitc cerviceque fusco-alboque. varlis: lateribus capitis curereis naculis mi.

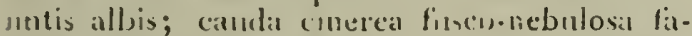
-riis 4 distantifus obscurefincis, aprice albo, -1: lotuis cinerascenltrallua olsolete fasciala. hites. I. c. A. Su. Zool.

Var. $e^{\circ}$ (f.) fuliginoso.fusca: marginibus pennartun dilutioribus; lateribus capitis cinc. reis albo immixto; sublus cincreo fulce: latchibus fuscis; abdrominc crisiog pe transwers et rarius alloo maculatis. Cetero ut ill var. $j$ Sv, Zool.

Var. s. capite colloque supra fermugiuea: punctis allbis uigricruce; fionte: all:da; Illd. cula per oculos fuscal; dorso alisqute obscure. fuscis: sulotus forringiueus: liness fuscis.

Nilss I. c D. Sy. Zool

jun. colore clirius brunnes: capite albo. et brumucomaculato; sublus rubrescente al. bus marulis maguis brumeis; pemis supü rubrescente marginatis.

Hab. minus frequens in sylvis, migralorius. Ailimalculis aquaticis et ifrrestribus. etjan insectis victitat. Nidificat in arboribus excelsis ova $3-4$ cineres maculis obscurioribuss ponens. - Lnngitudo 1 ped. ro poll. Rostrum rigrum plane edentulum, fere carinatum: cera fava. Iris luten. Caput el cervix fusco-alboque-varia; lateribus capitis cinereis naculis minutis albis. Dorsum \& alx supra obscure fusca. hemiges primores nigricautes, secundarii fuscilatere interine a basi albido-maculari. Macula subtus distantes rotundatre, gula tamen striis at crisso maculis iransversis fuscis. Cauda cinerea fusconebulosa: fasciis 4 distantibus pollicaribus obscure-fu. scis, apice albo, subtus cinereo-alba obyolete fascista; in var. $\varepsilon$ nigricans lineis Iransversis cinereo-rufis, pone apicem album fascia lata nigra; in juniore iterum nigrofusca lineis cinereis albisque. Tibix antice fere semiplumos $x$, dehinc lutex: unguibus nigricantibus.

$$
\text { -) tibiis plumosis: }
$$

3. lagopus: albo-fiscoque-varius: cippite, collo pectoreque albicantibus fusco-striatis; cauda alba, versus apicem album obscurefilsca.

Brünnich Ornitbologia borealis pag. 4, n, 15. Linn S.N. 
d. Gmel. 260, 58. Retz. Fn. 6. Nilss. Orn, 5. Fn. 71. 18. Falco. - Parel. Act. Nidros. 4. 419. 13. Palco Norvegicus Sv. Zool.

Sv. I uddfots Vråk, Gråfalk. - N. Graa. falk.

Junior: fuscus: marginibus pennarum albis 1. cinerco-albis; pectore, abdomine femori. busque albis, transverse fusco-maculatis; tibiis fuscis albo-undulatis caudayue alba nigro-fasciata: fascia extima látissima.

Nilss 1. c. Parel. 1. c.

Hab. in Lapponia non infrequens, $D$. Leem; sed alibi etjam in Scania, D. Retzius, rarior. Victus ejus $t d e m$ ac prioris. Vidun ejus in summis arborum prealtarum adifical. Clamorem $g$ u i, $g$ u i! sxe edit. - Longitudo circa 2 poll. Rostrum nigro-luscum, cero lutea. Iris feva. Regio inter nares el oculos albasetulis nigris. Capur, collum et pectus lutescemte-alba striis ad raches pennarum fuscis. Dorsim et ale fusca marginbus pennarum ferrugineisel albidis. Abdomen obsiure-fuscum maculis inserdum albis, (in mare lutascente albusm maculis obscure-finscis fere confuentibus). Remiges primores ab apice fere ad medium niwricantes intus albo-rsrii; secundarii fusci, basi albi maculis fuscis. Cauda alis complicatis paruin longior, supra obscure-fusca medietate interiore apiceque albis; sublus alba apice grisen: tectricibus superioribus albis maculis fuscis, inferioribus sordide-aibis. Femora sordide-alba maculis fuscis in mare transversis (in femine longitudinalibus): tibiis surdide-albis longitudinaliler tusco-inaculatis.

\section{G. 50. CIRCUS *), Sy. Ringhök.}

-) Hab. gener. fere prioris generis, sed corpore miniss robusto; alis longis: remige 30 vel 4. Iongissimu; chbis longit el gracilibus: unguibus sab lon. gis et aculis; culsuld longa rotundaca. - N. gen. Brissoni grecum: $\mathbf{S}_{6} 0 \% 0 \mathrm{~S}$, Accipiter annularus.
1. rufus: rufus: capite pectoreque superiore pallide-ferrugineis striis 1 . maculis longitudinalibus fuscis; cera viridescente-lutea; gula albidiore; cauda cinerea: lateribus fer. l'ugineis, subtus alba.

Brisson Ornithol. T. 1. p. 404 . - Linn. S. N. ed. Gmel. 266. 79. Nilss. Orn. 8 (jun. avis) Falco. $-S v$. Zool. $S v$ r ödbrun Ring hök, Hönstjuf. $-N$. Hönsehouk, Hönsebög, Hönsetyv.

Var. B. similis avi juniori, sed humeris nacula magna ovali rubrescente-alba.

Nilss. 1. c. Sv. Zool.

Junior: obscure-fuscus: occipite gulaque pallide testaceis, immaculatis; iride pallide brumnescente.

Linn. S. N. 130. 29. Fn, 66. Retz. Fn. 21. Hamm. Fn. 75. Nilss. Orn. 8. (adulius) Falco æruginosus. $S v$. Zool.

Hab. in provinciis astralioribus minus frequens. precipue in locis paludosis, ubi plelumque in orundinetis nidificat; var. $\beta$ in Uplandia, D. Thunberg; autume migrans iempore remali revertitur. Muribus, avibus minoribus, Jacertis, hirudinibus, ut et piscibus rescitur. lo terra fere semper sedit clamorem: $p$ its $p$ its! et piep piep! edens. - Longitudo circa 20 poll. Rostrum nigrum. Iris subaurantiaca. Regio inter nares et oculos seculis nigris. Scapulares et tectrices alarum apice oculos seculis ningris. ferrupinescentes; remiges primores nigri basi albid, Secundariu cum rectricibus cinereis. Al

2. cyaneus: grisescente-cxruleus 1. canus. sublus albus: or.hitis plumis capilis radiatis; remigibus nigris, rectricibusque fasciatis.

f. major fusca marginibus pennarum rufc. scentibus subtus rufescens maculis longitudi- 
nalibus fuscis; collare e pennis rigidis nigris, albo-marginatis.

Linn. S. N. 126 so. Falco cyaneus m., - n. I1. Retz. Fn. 9 Falco Pygargus $f$. -Nilss. Orn. 9. Fn. 76, 20. Falco Sirigiceps. Su. Zool.

Sv. blà Ringhök.

Var. $\beta$. fuscus: marginibus pennarum rufescentibus, fascia per oculos fusca albo-ter. minata, macula auris rufo-fusca, collare albo fusco-punctato; subtus albus rachibus penualium rufo-fuscis.

Nilss. I. c. D. Sv. Zool.

Var. $\gamma$. capite supra lateribusque dorso. que fuscis; stria supra maculaque infra oculos albis; remigibus fuscis fasciis obscuriori. bus latere exteriore cinereo; subtus testaceus. Nilss. I. c. Z. Sv. Zool.

Var. ঠ. capite supra, orbita, maculaque auris nigris; supercilia albida; collare ut in fem; dorso obscure-fuliginoso; specula alarum cærulescente; subtus albus: maculis fuscis, juguli confluentibus, cetero parvis raris.

Linn. S. N ed. Gmel. 277. 19. Falco Hudsovius. Nilss. I. c. F. Su. Zuol.

jun. capite supra fusco: marginibus pennarum ferrugineis, fascia supra infraque oculos albida, macula auris semilunari fuscescente; dorso alisque cinereis fusco-tinctis; subtus albus pectore fusco-striato.

Hab. in locis paludosis rarior, migratorios; var. $\beta$ at Uplandix, D. Paykull; iar. $\gamma$ Golllandix, Ipse. Nidificot io terra inter segetes 1 . in arundinetis ora 4.5 obscure carulescente-alba immaculata ponens. Muribus, aviculis, ranis lacertisque rictibat ob in terra sedens do-

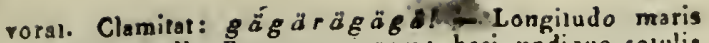
1 ped. 6-7 poll. Rostrum nigrum: basi undijue selulis nigris nares subtegenlibus; cera viridescente-lutea. Iris lutes. Remiges nigri ad basin albi. Couda supra cinerea apice albs. Pedes lutei. - Longitudo fem. I ped. 8.9 poll. Remiges extus fusco.nigroque,- intus albo-nigroque-rsdiati. Crissum album maculis rafescenlibus. Reque-radiati. Crissum nigrescenle-obscureque cinereo-, laterales fulrescente- el nigrescente-radiatz.

\section{G. 5r. FALCO $\left.{ }^{*}\right)$, Sv. Falk.}

I. islandicus: albus, supra tuansversim fusco-maculatus: cera pedibusque luteis; cauda alis longiore nigro-fasciata.

$f$. fusca, subtus albida amplius fusco-maculata: superciliis maculisque nuchæ albis; fasciis caudx luteo-albis.

Warhem. Act. Holm. 180x. 195 6. Brünn. Ora. 2. 7, 8. Bámm. Fn. 79. 6 F. - Nilss. Orn. 15. Fn. 8. 8 . Linn. S. N. ed. Gmel, 275. rox. F. candicans. Sv. Zool.

Sv. Is I ands Falk, Hvitfalk.

Var. $\beta$. capite colloyıte albis fusco-striatis; dorso alisque fuscis marginibus pennarum maculisque transversis albis; subtus testaceoalbus: striis maculisque fuscis; cauda cinerascente-alba fasciis angustis fuscis.

Nilss. 1. c $\gamma$. Su. Zool.

Var. $\gamma$. corpore cinereo alboque undulato, collare albo; cera pedibusque luteis.

-) Hab. gener. entecedentis sed alis longis acutiusculis: remige 3:0 longissimo; pedibus robustis paulo sub genu plumosis, tibiis cetero nudis reliculatis, digitis validis longis, unguibus curvalis ecutis. - Nomen geaer. lasinum estr 

Linn. S. N. 125. 7. Fn. 56. Retz. Fa. 8. F. rusticolus. Sv. Zool.

$V$ ar. $\delta$. fuscus fasciis cinereis, subtus albo. cineretıs maculis transversis fuscis; rectricibus extimis albis; pedibus luteis.

Linn. S. N. 130. 27. Fa. 64. Retz. Fn. 20. F. Gyrfalco. Sv. Zool.

$V$ ar. $\varepsilon$. capite colloque ut in var. $\delta$; sed dorso alisque cinereo-fuscis albo-fasciatis; sub. tus albus striis jugnli maculisque lateralibus pectoris abdominisque rotundatis 1 . transversis fuscis; cauda cinerea fasciis angustis fuscis. Nilss. l. c. ß. Sv. Zool.

Var. $?$. cinereo-fuscus, sublus albus striis maculisque longitudinalibus fuscis: cera lute. scente; pedibus plumbeis; cauda flavescentefasciata.

Billb. in. Act. Holm. 1809. 202, F. umbrinas. Svi: Zool. $H a b$. in sylvis Norvegize hyperboreis non infrequens. Svecize vero rarior et var. $\beta, \varepsilon$, et $/$ rarissim $x$, unde vix varielates $x$ latis "\% Inter congoneros, pro renatu fere omnes aptos, $\boldsymbol{P}$. islandicus Falconariis principaliter estimatur. Nidificat in arbore excelsa ova 3.5 maculata ponens. Mammalia porra, quz agiliter et fere perpendiculariter aggreditur, et ares majores cibus ejus sunt.Longiludo maris I ped. 9-10 poll. Rostrum lutescens apice nigricante. Iris brundescens: orbita lutescente. Albus, pennis supra anguste brunnescente-lineatis, infra maculis brunnescentibus gutlitormibus, hypochoudriąrum

\footnotetext{
-) Hre varietaces authoritato D:m Temminck et Nilson relabe, quamvis dubimus de qua jure; quum nec colorem cera peduinque, nec formam colo. remque mecularum ef fasciarum corporis caudave, ut cheracteres constontes approbant. quosnem speciem deberminandus nescimus.
}

majoribus et numerosioribus. Pades lutei. - Longitudo fem. fere 2 ped. maculis infernis numerosioribus fuscis et in hypochondriis confluentibus, fascias fere formantibus; lineis supernis eljam latioribus et pluribus fuscis.

2. lanarius: cinereo-brunneus, subtus albus maculis lanceolatis clare brunneis; super. ciliis maculisque caudæ ovalibus rubrescente. albis; rostro pedibusque cæruleis.

f. major: vertice brunneo-fuscescente, maculis subtus lanceulatis latioribus striisque ạn. gustis gulæ crissique fuscis.

Linn. S. N. 129.24. Fu. 62 f. Retz. Fn. 19. Homm. Fn. 72. - Sv. Zool.

Sv. Ullfa lk, Blåfot. - N. Smirle.

Var. $\beta$. fuscus supra, mactilisque cauda. libus transversis vel rotundatis.

Nilss. Drn. 17. Fa. 15. 2. - Linn. S. N. ed. Gmel. 274. 95. F. stellaris.

Hab. in Scania passim, in Norregia frequentior, migratorius. Avibus majoribus raro mamnalibus victitat. Nidificat in locia molltosis inter frutices. - Lngitudo maris 1 ped. 7 poll., fem. I pad. 8-9 poll. Cera, iris et orbita oculorum lurea. Verrex clare ruber, maculis oblon. gis brunneis. Supra cinereo-brunneus penais clare rubro. marginatis; maculse inferne deorsnm latiores et longio. res. Gula crissumque immaculacs.

3. peregrinus: cinereo-cxuleus fasciis obscurioribus; capite colloque supra nigre. scente-cxruleis; sthtirs albus st ris maculisque butuneis; inacula ab angulo rostri cxrulescen. tis descendente nigin; pedibus luteis.

$f$. major: colore sordidiore et sublus ver. sus inferiorem rubrescente-a!bo.

Linn. S. N. ed. Gmel. 272. 88. Wachem. in Act. Holm. 1802. 256. Nilss. Oril. 16. Fn. 18. 5. - Sv. Zool. 

Sw. Va rdringsfalk. Vallök.

annotimus: cineseo-fuscus: marginibus pennarmm chsolele firmginescentibus; subtus testac(o)-albus: pectore, abdemine, crisso femo1:Lusque maculis longitudinalibus fusco-undillatis.

Nilss. I c. var.

Jun. lincus: mavginibus penuarum ferme gincserntibus; subtus testacen-albus: pertore abduminergue maculis longiturlinalibus fuscis; crisso pallisle testaceo transverve finsco-lineate. Linn. S. N ed. Gmel. 270 86. F. colnmunis. Nilss. I. c. Hal). per lolam Scandinaviaul sat rarus, migralurius; pro venasu valde expelendus. Nidificnt in rupibils, ex. $g^{r}$ in iupe, Wallall, prupe urbem Carlshamu, $D$ Nilsson; ubi ova 3 a rubiescenle-lutea maculis brunneis ponit et ce; limanas 3 incubut. Clamal gein, gris! - Longitudo m. 25 poll $f$ i7. pol!. Rosirum undentalum: Cera, oibita oculi el iris luter Gula at pectus alba siriolis sparsis, et ahdomen sordide-slbum msculis transtersis brunneis. Tiemiges illus maculss numerosis bulnuescennbus el albielie Conda brevur , bscure sinerea lineis 8.10 transvarsis ligricanlibus a pice abo. Pedibus luteis a pras. cedente, quo simıllimus inprimis in asape juniore nox cognilus esl. D giti pralongi.

4. subfuteo: cærnlco-fiscus: capite nigricante lumula sub oculis nigra; subtus albus: naculis longitud.nalibus nigris; femoribus crissopue ferrngineis; caula bievi.

f. colore supra obscuriore sublus sordidiorc; vorculis brumsioribus; crisso femoribusque muins clare ferrugincis.

Linn S. N. 227 4. Fn. 50. Riecs. F11. 14 Nilss. Orn. 18. Fn. 21 4. - Sv. Zoul.

S2. Vrakfalk, Lärkfalk. - N. Lerkefalk. jun. obscurc.fuscus: marginißus pennarum ferruginescentibus; subtus tentaco-albus: maculis longitudinalibus fuscis.

Hab. in sylvis sol frequens, cum A!auds arrensi o. migrat el fele reverlilur. lia el avicul sull rapiua, quam sub volasu seclalur. In aboribus nidifical uva $\mathbf{3} \cdot \mathbf{4}$ solundala cxerulescente-s!bo maculis grisws et olivaceis ronens; resperibus clnmilat: jäok, jä̈ö, jäök! Longiludo y ped. a poll. Kusirum creiulesceus: cera lule.." Llis fusca. Canut et collum nigricaules cineren rufugiı lincso. nucha maculis 2 albn-rufescentibus. Keniges lalere inleriore maculis 7.8 "Ianyversim ovalibus rufescenle-albis, primores nign, secundarii fusco-cinte $\mathrm{i}$ margine apicis pallido. Coiuda aequalis: reclricibus anereo-lusris, larere interione fusciss 8-ro rufo-albs. Crissum femoraque forruginea. Pedes pallide-lavi; unguibus nigris.

5. liclunfalco: carmlen-cinereus: rachibus nigrie; sulutus albidus maculis longitudinali. bus fuscis; canda longa: fiscia pone apicem alt)!:ı nigra.

f. supra colore obscuriore subtusque lutescente: ubi mas formentucus, maculis infernis majoribus at mumernsioribus.

Linn S. N. ad Ginel. 278. tus VOachem. in Act. IIolm. s\$02. 261). Liet $=$ 111. 15. Nilss. Uru. 19. Fn, 24. 5 (m.) Linn I. c. 234. 118. F. xesalon (f.) Sv. Zool, Sp. Steufalk.

rnnocinus: sujpa olscure brunneus marginibns peonarmin ruberscentibus; cauda ni-

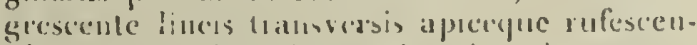

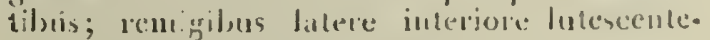
albis maculis longritudinalibus bionneis.

ISab. in sylvis montosi, Iariur, megratorius. Aviculis

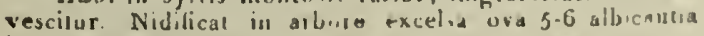
ill extromitale visidescenlo-Lrunueu marmorata pollons. - 
Longiludo m. II $f$. 12 poll. - Rosirum crerulesceis : cera lucea. Iris in. lulea, $f$ fuscescente Lalera capitis. supercilio et cervix ferrugineo-alha : maculis el striis fuscis. (Dorsum et incirices a'drum fem. eljam maculis iransverss et opicibus penuarum obsolete-lerrngineis). rula alba immaculota. Remiges nigricantes lalere inleriore maculis albis. Cauda cinereo-carulea lineis 4.5 irans. rersis obsolecis fuscis, (fem. 7 albo-lutescentibus). Crissum femoraque albida siriis sparsis fuscis. Pedes fari: digitis sublus nodnsis, unguibus nigris.

6. cinnunculus: capite colloque cinereis rufo-tinctis; striis fuscis; dorso alisque rufis, nlaculis uigris; cauda longa fascia lata pone apicen album nigra.

f. rufa: capile colloque striis, cetcro ma. culis trausversis fuseis; subtus albida: pectore ablomincyue testaceo-tinctis, longitudinaliter fusco-maculatis.

Linn. S. N. 127. 16. Fn, 61, Retz. Fn. 18. Nilss. Orn. 20. F11, 28. 6. $-S_{21} .100 l$.

Sv. Klockfalk, Kylhfalk, Tosnfalk. $-N$. Kurkefails.

jun. testacion-fuscoquc.fasciatus, sublus albidus maculis longitudinalibus fuscis fere conHineutibus.

Nilss. 1. c. - Retz. Fu 17. F tascialus.

Hab. in currabus, ruderis nreallis non infrequens, in arboribus rarior, ubi nidulatur el uva 5-arubrescenlelures major bus 1. minoribus maculis rubrescenle brunneis, inceiduon locesicia maculis obsculioribus pontl. Mam. malia parra, avicula, amphibia, insectaque adgredilus \& prodalur Sonital $k l i k l i k l s$ et $p l i p l i p l i p l i$ ! - Lanpribulo $\mathrm{m}$. If $f \quad 16$ poil Rusirum cierulescens, basi llovicalls: cera lucea. Iris firsca. Urupy gium cinereuin. Subius pallide-iufus: stria a sinu oris ad lalera juguli negricuste. Gula crissumque immaculala. Peclus \& abdomen maculis porris oralibus nigris. Kemiges u- gricantes lalere interiore maculis transversis albis; teclricibus inferioribus albis, maculis subcordalis nigris. Cauda cinerea, ifem. rula fasciis fuscis, quarum ullina lalissima). Podes lutei.

Nat. 16. STRIGIDES,

G. 52. STRIX *), Sv. Uggia.

") capite lavi.

$\dagger)$ apertura aurium minore orali: ")

1. passerina: fusco-cinerea: punctis $\mathrm{ma}$ culisque albis, subtus alba: maculis longitudinalibus fuscis; cauda lineis \& transversis apiceque albis; pedibus ad ungues dense plumosis.

$f$. obscurior: maculis supernis alhis lutco tiuctis, infernisque fuscis distinctioribus.

Linn. S. N. 133 12. Fo. 79 . Retz. Fn. 86. Tengmalmz in act Holm. 1793. 294. Hamm. Fn. 85. Nolss. Orn. 78. t. 3.?' Fn. 90 4. - Rudb. Ar. pict. (De Geer) 165. Sv. Zool. 56.

Sv. S par fuggla. - N. Katugle.

Hab. in sylvis haud frequens. Niuribus ariculisque rescilur, pellem primum amuvens, insecta tamen dirersa non respuit. Nidificans in arboribus cavis $/ \mathrm{em}$. ova 4 -lba ponit. Sibilat: kirr! kirr! - Lungitudo m. 6. fein. 7 poll. Rostrum viridescenle-luteım, apice pallidiore. Ins crocea. Celero - diagnosi bene connila.

2. psilodracıla: griseo-fusca: punctis ma.

-) Hab. gener. Corpus fere obconicurs: alis aculiusculis, remige 3:0 longissimo; pedibus robustis, digitis 3 anticis, externo versatili.

-1) Strigides hujus sectionis proxime falconibus sesc epproximent. 


$$
-1-
$$


culisque albis; subtus rufescente alba: macu. lis longitudinalibus fuscis, collo albo imma. culato; cauda lineis transversis interruptis; digitis sparse pil ssis.

$f$. colore sordidiore colloque rufescente maculato.

Recz. Fn. 55. (Descriptio) Sir. Noclua. - Nilss, Orn, 30. c. 2. Sir. Iludipres. Fn 88. 3. - Sv. Z0ol.

S2. Finfotsuggla, Perluggla.

Hab. in proviurcis meridionalibus rarissima, $D$. Tengmalm, in Europa australi sal frequens, rerisimilier avis Mlinesve. Mures, respertiliones, avicula, inseciaque varis ubum ejus probellt. Nidulalur in cavitatibus muturilin, raro arboulum, ora 2-4 alba ponens Clamitat: p 1 p :, pupu! el injerdum miser: kliat klivic! I) ngitudo 8.9 poll Kostrum flarescens, Iris hava. Prz. cedelli speciei valde affnis, sed digilis pilis rarioribus adpressis insıruclis, lineisque transversis rectricum racbi ioterruptis hiox diversa.

3. ulu!a: fusca: maculis albis; subtus al. ba: fasciis trausversis fuscis; cauda longa cuneiformi fusca: linte is transversis apiceque albis.

f. majol: colore sordidiore.

Linn S. N. 153 10. Fn. 78. Nilss. Orn. 28. Fn. 85. .. Homm Ful. 84 Tengm. in act. Holm. 793 . 284. Piecs. Fn. 53. - א2. Zool.

Sv. 'T jutuggla. - N. Fieldugle.

Ilab. In syivis densis provincianm septentrionalium sed minus fieyuens, preserim Vesiruboltniz; ravior ad prublacias inferioros migrans. Cit priores, nuribus, aciculı insectisque riclitat. In arboribus nidificat et ova 2 albs pouit. - Lonsiludo $14-16$ pull. Kostruin lutein. suitus ngricans. Ilis claro-lulea. A prioribus diffell: liisgnilud ur, fasils inferais el cauda longiole haud iransrer.e-linevit. of regiode circa areas oculoruin densius albo-niaculalai s Sor. nocius eliam: pedibus ad ungiles usyue deusa pluinosis.
4. uralensis: grisescente-alba: maculis longitudinalibus distantibus fuscis; area oculorum pentis rigidis albis nigro-maculatis cincta; cauda longa.

$f$. colore sordidiore.

Linıl S. N. ed. Gmel. 295. 35 - Retz. Fn. 29. Nilss. Oin. 25. Fn. 96. 6. Tengm, in act. Holm. 1793. 267. Str. lituraca. Su. Zool.

Sv. Urals Uggla.

jun. supra clarius brunnea, cinereo-brunneo testaceoque variegata maculisque intermixtis ovalibus albis; subtus maculis cinereo. brunneis.

Meyer Taschenbuch Deulschl. I. 84. Str. macroura.

Var. ß. albida: Inaculis striisque clare brunneis.

Sparrm. Sr. Ornithologi lab. Strix Lindrothii.

$H a b$, in Lapponia, $D$. Lindroth Mammalibus ariculisque vescitur. Nidulatur in cavis arborum, ova $3-4$ alba ponens. - Longiludo I ped. 8 poll. Caput grande: facie lata. Rosirum favescens. Iris brunnea. Macula supra longitudinales sublus lineares. Kemiges recticesque fasciis brunneis. Pedes albidi: tibiis digitisyue dense plumosi, usque ad ungues longissimas lutescentes, punctis mınutis brunneis.

5. nyclea: tota cum facie nivea immaculata: capite parvo; cauda alis parum longiore.

f. nivea: maculis parvis transversis sparsis fuscis.

Linn. S. N. 132. 6. Fn. 76. Rets. Tn. 28. Thunb. in act. Holn. 1798. 184. Hammi Fn. 83. Nilss. Orn. 56. 25. vor. f. Fn. 82. x. - Sv. Zool.

Sv. Sk y maingsugg la, Harfâng, Snöuggla, 

Fjälluggla, Fjällberguf. - N. Leniens. griis, Gysfugl.

Jun. alba: maculis najoribus.

Var. ß. alba: apicibus remigum $1: x$ et $2: x$ macula transversali fusca.

Nilss. I. c. $\gamma$.

Vor. $\gamma$. alba: capite supra maculis parvis dorsoque undulis racioribus fuscis; cauda remigibusque apice fusco-maculatis; pectore un. dulis rarioribus obsoletis.

Var. \&. alba: capite supra maculis, dorso caudaque versus apicem fasciis, subtus undulis fuscis.

Nilss. I. c. $\alpha$.

Hab. in alpibus lapponicis, ubi nidalatur, ora 2 alba ponens; migrat rero aulumno per paria al regiones australiores. Lepores, Hypudai boreales, Mures, Telraostralores. Libus ejus sunt. Bonus ejus stridori suis similia erit - Longitudo 2 ped. Rostruni nigrum. Iris clare arantiaca. Cauda rotundala, unde Sir. uralensi mox distincsa. Pedes ad ungues asque densissime plumosi.

H) aperlura aurium semicirculari.

6. lapponica *): fusca: undulis flavescentibus albidisque; subtus albida lineis trans. versis maculisque longitudinalibus fuscis; facie

-) Neutiquam D:o Nilsson hanc speciem Sirigem nebulosam esse, consentire possumus, uspoce apice caudx albo bene distinctam, quiéque prceterea. est americano, de cetero eandem esse speciem D: $\mathrm{i}$ Temminck hujus nominis credimus, at vero eans habere audemw sententidm, quod proxime laudatus auctior minus recte existimet Strigem stebulosum europaem. cinerascente lineis concentricis arcæ oculorum fiscis: cauda apice fusca.

Sparrm Sv. Ornilh. Retz. Fn. 50. Nilss. Orn. 21. Fn. 93. 5. - Sv. Zool.

Sv. Lappsk Uggla.

Hab. in Lapponia superiori. D. Sparrman, manens. Vitz genus degenda adhucignotam. - Longitudo a ped. - poll. Caput grande: rostro lutesconte-albo, iride flara; maculze pectoris transversis linexformibus, abdominis longitudinalihus. Cauda alis longior cinereo-fuscoque variegata apice lusco, sublus albida fuscescente-fasciate. Pedes albidi fusco-undulati ad ungues usque dense plumosi.

7. aluco: griseo-ferrugineo-fusco-nigroque varicgata: capite prægrandi, iride atra: alis albo-maculatis; margine ciliatis.

$f$. colore magis ferruginescente iride brunnca. Linn. S. N. 13\%. 7. Fn. 72. Retz. Fn. 3r. Tengm. in acl. Holm. 1793. 270. Rudb. Ar. (De Geer). 171. Nilss. Orn. 26. Fn 98. 7. $\rightarrow$ Linn. S. N. 133. 9. Fn. 77. Str. stridula, f.? Hamm. Fn. 82. Str. funerea. Sv. Zool. Sv. egentlig Uggla, Haruggla, Kattuggla, Skrikuggla. - N. Krakugle.

Hab. ubiqne vulgatissimus. Vescitur mammalibus avibusque diversis, ranas vero insectaque majora non fastidit. In nidibus avium aliarum ova $3-5$ oralo-globosa albida ponit. Mas criat: $h u h u h$ it $h u h u$ ! territus eljam $k a ̈ c k$ et kackib! sed rox fem. stridulans est. - Longitudo maris 15. fem. I4 poll. Rostrum apice flavescens, basi carulescens Area oculorum cinerascens rufo-inmixto. Cetero diagnosi bene cognica.

8. funerea: olsscurc-custanea maculis punctisque albis; subtus albida maculis fuscescentibus; cauda per paria albo-maculata; prdibus totis dense plumosis.

$f$. inajor: colore supremo magis grisescente; macula inter oculos et rostrum atra. 

Linn. S. N. 155. I1. Fn. 74. m. 75.f. Rete. Fn. 35. (Diagnosis; Nilss Orn. 29. Fn. ror.8. Rudb. Ar. picl.(De Geer.) 165. m., 170. $f$ Noctua major. Tengm. in aci. Holm. 1795 . 289. Sir. noclua. Sv. Zool.

Sv. Likuggla,

Hab. in sylvis densis seplentrionalibos minus frequens. Cibus ejus lalis ul pracedentiun. Nidulalu: in pinetis ora 2 alba ponens. - Longitudo maris 9. fom. $9 \frac{1}{8}$ poll. hostrum flarescens. Area oculorum alba: lineolis sparse nigris, (in fem. macula atra oculos fere circumdante), iride lutea niteute. Aifinis certissime Str. passerina, psilodactyla sed magniludine semper majore et differenlia in diagnosibus indicata suffrcienle.

-) capilo aurilo.

9. Alammea ${ }^{\star}$ ): ruber: maculis fuscescen-

-) Ut hac landem constituatur species et suomet primo nomine nuncupetur, non possumus ron, quin ejisdem rationem exponamus: Strigem flammenn eodem planemodo in Systemate ac in Fauna, in qua etjam descriptio haud ambigue sumba est e.t jigurn in avibus pictis Rudbeckianis Cel. D. r. Linne descripsil; hanc nullam esse aliam quam Strigæm brachyotum D:orum Temminck et Nilsson seu Strigem brachyuram D:i Nilsson, in Ornithologia ipsius, satis etjan constat, quam ob caussam permusacum nomen flammex sine dubio huc pertinet. Causso, cur permutatio nominis facta sit, est quatrenda iu eo, quod huic in Systentace speciei sy. nonyma Linne attribuit, qua non ei, sed in scan. dinavia prorsus peregrincs speciei, cui, ad confu. sionen diutinam denique evilandam, nomen Gesneri, descriptor hujus primus, propono. Hace a nostra diversa est: cólore fundamentali magis in luteo vergente, et supra puncsis nigris, alque un. dulis cinereis fuscescentibusque. Speciem nostram ad hanc sectionem autoritale D:orum Temuninck et Nilson retulimus; attomen nondum nobis satis tibus, subtus incarnata striis pectoralibus fuscis: capite parvo; rostro orbitaque nculorum proxima nigris; cauda brevi fusco-fasciata.

$f$. maculis minus obscuris.

Linn. S. N. 135 8. Fn. 7j. Rudb. Ar. pict. (De Geer.)

- 167. Sparrm. Mus. 51. Str. arclica. Brünn. Orn. 17. Str. Aluco, Nilss. Orn. 27. Sir. brachyura. Fn. to6. II. Sir. Brachyotos. Sv. Zool.

Sv. Branduggla.

jun. facie fere tota nigra.

Hab. in sylvis Lapponicis sat rarus, ad prorincias ausiraliores interdum-aulumno migrans. Mammalid minoria cibum ei prebent. Nidificat intra gramina in cxes. pite? - Longiludo 13 poll. Rostrum nigrum apice pallidiore. Iris flava. Alx albido-maculalx. Collum colore dorsi fusco-maculalum, sed peclus, abdomen, crissum pedesque incarnala striis in peciore fuscis subcruciatis. Affinis quidem sequenli sed characleribus diagnosis valde dirersa."

10, otrs: fulvo-fusco-albidoque variegata, subtus lestacea: maculis striisque cruciferentibus fuscis; pennis aurium longis nigris; cau. da alis breviore.

$f$. colore grisiore, gula area oculorum fa. cieque margine fusen-macnlata albidis.

Linn. S. N. 132. 4. Fn. 24. Retz. Fn, 26. Nilss. Orn. 22. Fn. 107.10. - Sv. Zool.

- Sv. Ör e ugg la, Hornuggla. - N. Hornugle. jun. pallide-ruber: lineolis transversis nigricantibus; alis caudaque griseis punctis brun.

est persuasum de existentia plumarum aurium lon. giorum, quamvis plures species noortuos inspeximus. D.i Tengmalm et Retzius Strigem Gesneri descripserunt, presumentes eam esse lammean Linn. 
neis fasciisque fuscis; area oculorum obscure fusca.

Hab. ubicunque in sylvis haud infrequens. Muribus inseclisque victitat. In alsenis niubus lein. 4-5 ova subflobusa alba ponit. Criat: $h u u c k, h u u c k$ ! - Longitudo 14 prill. Rostruin nigrurı, Iris aurantiaca: orbila ad pennas a irium adsceudente nit.ra. Area oculoruin teslacea : margine pesuis nigris pice luteo albuque variegalls cireumdulo. Reunigas primotes rufescenies luscon-, ad apicen cinereo-fuscofuefarciáti. Cauda rulescenteresticea allguslius lusco-iasriata, apire cirerascenle. Abdomen pedesique restacera, imniaculala.

11. Brto: testacon-nigro-ilbidoque varie. gata, subtus testacea: naculis longitudinalibus nigricantibus; pennis amium lougis ple. rungule nigris; canda alis longiorc.

$f$. ruajor: culore pallidiore, gulaque hand alba.

Linn S. N. 131 1. F॥ 6g. Rets. Fn. 21. Tengm. in sct. Holm. 1795.256 Hamin. Fu. 8t. Nitss. Oril. 21. Fa. 10.6. 9. - Sv. Zool.

Sw. Ufuggla, Hormuf, Berguf. - N. Bierg. ugle, Inorumgle? Rnper, Hujogosten.

Var. $\left.\beta^{*}\right)$ : alba: maculis nigris allspersis.

Cirn. S. X. :52. 2. Fn. 70. Ret3. Fn. 25. Sirix Scandiaca. Risdb. Av. pict. ol. Bubo scandianus. Sv. Zuol,

-) Hanc, unam rarissimarum scandinavis avium, $e$ Fasna nostra excludere passumus, cum tain ceitn moido D:s v. Linne eam denotavit, sed heic loc'un: ineepin tenere zolubinus. Rejerre Strigem Scandia. ram liun is Strigem nyitesn, nec converat ei. quvid Linne secunidum figuram Rudherki ei iaput auritum expresse atcribuit, nec lis.gnitudine, qua esset eadem ac Meleogridis (Pseudutaonis Sylvaticis). quibus utrisque datis notis, non potuit non Linne susin valde cognifom Sirigem dycteam disbrnguer.
Hab. in montibus prealtis, ubi nidum e virgis frondibusque edifical latitudine 3 ped. ova $2-4$ alba ponens. qua septimanas 3 iucubat. Mammalibus minoribus, avibus mediocribus et amplibiis diversis non solum rescitur, sed eijam mammalia aquilasque aggreditur. Clamorem horriduin perpetuuin: $p$ us $p u h$ ! edit. - Longitudo maris 2 ped fem. 2 ped. 4 poll. Rosirum cxruleo-nigricans. Iris Sulva. Maculx infernze longitudinales nigricaples margine variegatie. Crissum, tectrices caudx inferiores pedesque fusco-undulata. Cauda teslacea fuscofasciala et variegata: recirices 2 intermediis obscure-fucis maculis punclisque albidis raniegalis.

\section{Ord. 3. ANECOPODES, Trib. I. MADORHAMPHAE, Nat. 17. CUCULIDES, G. 53. CUCULUS *), $S_{\nu}$. Gök. \\ - $\quad$ tibiis brepioribus.}

1. canortes: obscure cincreus: pecture abdomineque albidis, nigricante undilatis; canda nigra: maculis albis.

f. minor: collo subtus cinereo, lutco inmixto, abdomineque sordide-albo fusco-undulatis.

Linn. S. N. 168. T. Fn. 96. Retz. Fn. 5o. Hamm. Fn. 97. Nilss. Orn. 57. Fn. 5 25. Rudb. Ar. pict. 159. Sparrm. Sv. Ornith. 1ab. - Sv. Zool.

Sv. Galgök, Gauk, Kuku. - N. Giög, Gouk. Var. $\beta$. similis var. $\alpha$. sed pennis super-) Hab. gener. Corpus oblongum : alis mediocribus.
remige 3:0 longissimo; cauda longa, rolundalo: pedibus scansoriis digibis divisis, experiore possicu versasili. 

nis rufo-maculatis alboque marginatis; cauda rufo-nigroque-fasciata maculis albis.

Rudb. Av. picl. I6o. Sv. Zool.

Var. $\gamma$. tota brunuescens: subtus vero al. bicans; apicibus remigum albis; cauda nigrofasciata maculis albis.

Sparrm. Sv Ornith. tab. C. canorus var. Sv. Zool.

Var. $\delta$. rufescens, subtus albicans, totus nigricante-undulitus; cauda: maculis, fasciis 4 ad rachin angulatis, fasciaque poue apicem album latiore transversa nigris.

Sparrm. Mus. 55. Sv. Orn. C. hepalicus. Nilss. Orn. 5s. C. rufus. Sv Zool.

jun. cinereo-fuscus: apice penuarum albo. marginatis collo tectricibusque alarum trans. versiu rubro - variegatis inacula subfrontali alteraque muchali alba; subtus albidus fuscoundulalus cauda olsolete rubro-nigroque-fasciata.

liudb. Av. pict. $\mathbf{x} \mathbf{x}$.

Hab. Wbique in sylvis, passim in horlis sat vulgaris: varielates vuro rarinres; anne ha, var. $f$. excepto, varieates lanium atatis? Insectis, larvis, vermibusque vicli1at. Fem. ova 6 grisescente-alba maculis brunnescentibus in terra ponil, ea postea inler unondibulas in nidisavium aliarum antes prefixis ovum unicum transportat, ubidies 8 nucrix aliena, munquam ea ipsa incubat. Reduces circa 4 diem Maji. smores ad medium Julium et juniores a finen Augusti in Africam emigrant, insulam Moltam jierumijue bis per annum obvientes. Clamor ejus monolona: $k u \cdot k u$ ! in $G$ et $E$. instrmmentorun bene cognila est, qua: cessil edere mense Julio. - Longitudo m. I 4 . f. 15. poll Kostrum nigrun (junioris fuscum) sublus virescens. Iris Aara. Cauda rolundala: rectricibus, quarum extima ceteris multo brevior, to nigra apice lbn, incesmedis 2 maculis juxta rachin partis albis. Pedes Davi.

\section{Not. 18. PICIDES,}

G. 54. JYNX *), Sv, Tyta.

r. corquilla: grisea: maculis punctisque nigris, rufescentibus albisque, tænia occipitali et oculari fusca, subtus Havescente-alba: lineis transversis maculisque triaugularibus nigris.

f. colore pallidiore; tænia occipitali brevi.

I inn S. N. 172. 1. Fn. 97. T. I. f. 97. Kudb. Av. pict. 117. Retz. Fn. 52. Nilss Oin. 56. Fn. 3. 22. IIamm. Fn. 98. Sporrm. Orn. lab. - Sv. Zool. So. -

Sv. Vändhalst y la, Göktyta, Sädgök. $-N$. Dreihals, Saagotik,

Hab. ubique frequens. Redux diebus paucis ante Cuculum canorum, vulgo signum semendi, emigiat autumno lempestivo. In cavis arborum pulridorum ova 6-8 lactea sine nido ponit. Inseclis pracipue formicis victitot. Ad objectum minulissime alienum caput reverse et utrinque torquel, cristam irata erigens. Suidulal sirpe tempore vernali: $g^{i} g^{i} g_{i} !$ - Longiludo 7 poll. Rosiruin griseo-fuscum. Iris luleo-brunnea Trenia ab occipite ad medium dorsum nigra ferrugineo-varia alterago in lalere capitis ab oculo fusco-rulescens. Rrmiges obscure-fusci: latero exleriore fasciis ferrugineis et nigricantibus, margine interiore ferrugineo.albo maculato; intimi dorso concolores. Carida longa rolundala, cinereonigroque lenuissime variegata lineis 4-5 transtersis nigris, quarum inleriores laliores; reclricibus 12 oblusis, quarun axlina utrinque brevissima. Pedes plumbeo-virescentes.

\section{G. 55. PICUS **), Sy. Hackspik.}

-) Hab. Gen. medius inter Certhiam ec Picum: alis mediocribus, remige $2: 0$ longissimo; pedibussca:zsoriis, digitis anticis ad basin unitis, posticis divisis.

-) Hab. gener copise collo majore: alis mediocri- 

-) pedibus h-dacıylis,

t) co!ore principali viridescente.

I. viridis: viridis: pileo coccineo, regione 1):?:!eriin nimra.

f. pleo coccinea angustiore, regione nigra minulc mýtilecisque nigris.

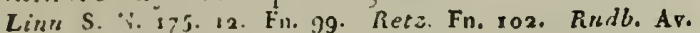
pirt. (De Geer.) 124, $f$ 125. m. Sparrm. Sr. Orn. 7. h. Ilumin. Iin. Iuo. Nilss. Orn. 49. Fn. 5. 6. 2.Sv Zonl

Sv. Grün IInckspik, Grönspell, Grönspik, riöngölling, Gyllemiuna, Vedknarr: N. Grï Sprel, Gul Spxt, Grönspette, Cinlspictte.

jun. peunis pilei ponc apicem coccineum macula ad rachin nigra basique cinereis; pennis dorsi undula alba; uropygyio obsolete álbo. fuscogyc-usululato; sublus sordicle-albus, nigro-unaculatus.

Hab. Fere ubique in sylvis truncos scandens pro caplura lurvaruni pracipue coleoptrorum et in terra curreus formicas quarere. Ora 5.8 alba in cavis arborum rrofisndis fesn. ponil: Clnmitar sxpe $g a ̈ r k, g a ̈ c k !-$ Longitudo 85 poll. Rostrum cerulev-nigrum, basi subtus livescens. Iris alba. Capur a fronte ad nucham coccineuin, basi peanaruin glauco intarlucente. Regio ocuforum intedum maculis parris a!bis. Collum, dorsum ot: uectrices alirum olivacen, uropygio splendide viride1!sro. Gula albicans. Crissum \& abdomeñ presertim ad

bus, remige 3:0 $2.4: 0$ longissimo; cauda plerumtue fuscuia, rectricitus 12 raro 10 extimis brevissimir, intermediis lungioribus, rigidis, acumina. is: ;redubus scansoriis, digitis 2 anticis basi co- duntutis, 2 posticis, raro unico.
Jarera luteo-viridia. Remiges nrimores maculis luteo-slbis. Rectrices 12 , quarum exrima brevissima lisca: margine upiceque obluse olivarea; cerera acure niçricantes lineis Iransversis virescentibus ubsolelis. Pedes siridescente-plumbei.

2. canns: viridis: capile collo corpore. que subtus cincreis; fasciula inter nares ocu. losque nigra, aliaque ad latera juguli interrilpla.

Lirn. S. N. ed. Gmel. 454. 45, - Rudb. Ar. pict. t. $\mathbf{1}_{2} \overline{3}$. Retz. Fn. 54. P. riridıs B. Hamm. Fu. roo. 6. Nilss. Orn. 50. P. caniceps. Fn. 3. 9. 3. $-S_{2}$. Zool.

Sv. Grî IIacks pik. - N. Graagrön Spxt.

Hab. imprimis in regiombus septentrionalibus passim, D. Rudbeck fil.; moribus ciboque antecedentis. Longitudo 12 poll. Rostrum cxerules-fuscum,-sublus ad basin viride-favun. Iris rubesceure-alba. Reniges fusci : latere interiore ransversinı albo-naculati; maigine exteroore maculis minoribus luteo-albis. Corpus subtus pailide-cinereum nlivacen-tinclo gula pallidiore. Crissum et teclrices caudx inler. sordide-olivacea; robsolete fuscoundulata. Re:lrices nigro-virides: intelmediis 2 obsoletelascialis; subtus 2, 3 apice exlus sordide-ıncane, 6.6 lutescente albis apice treniaque ad rachin dentate nigris. Pedes cinereo-virides : unguibus griseo-luscis.

$t \dagger$ ) colore atralo:

3. martius: atcr: pileo coccinco.

$f$. colore magis infuscato occipileque tantium coccilleo.

Linn. S. N. r73. 1. Fn. 98. Retz. Fn. 53. Nilss. Orn. 48. Fn. 3. 3. 1. Hamm. Fn. 99. Sparrm. ST. Orn. 8. h. Sv. Zool.

Sy. M als Iackspik, Spillkuáka, Tillkrâka, Toile.-N. Sort Ilakkespet, Sort Truekloppe, Sort Spat.

Hub. in sylvis sat frequens, permanens. Colenpleris. larvis eorum baccisque diversis ristitat. Clamitat ruians 
Gir, gir! et sedens ism glick, glick, jam turror - liuu! ora 3-4 candida ponit - Longituda 17 poll. Rostrum ad basin albidum, apicem versus cxruleo-luscum. Iris pa!lıde-flava. Totus aler: capile matis a fronte ad oce put coccineo.

4. leuconocus: albo nigroque varius: fronte albida; pileo rubro; dorso josticc a!bo; pectorc abdomineque albidis striis nigris; crisso rubro.

$f$. pileo nigro.

Rudb. Av. pict. ( $D=G e e r)$. 12t. $f .122$ m. P. varius ma. jor. Nilss Oris: 51. Fn. 3. 11. 4. - Sparrm. S r. Orn. 7. W P. major. var. medius. Su. Zool.

S7. Hrity y IIacks pik.

Hab. in sylvis minus frequens, $D$. Rudbeck fil. Moribus victuque congenerum. Ors 4.5 alba ponit, - Longitudo rot poll. Kosiruin pallide-cxruleum. Iris purpurea. Iaters capitis collique el gula alba: macula triangulari nigra. Supra nigra: dorso postico uropyginque - lbis. Pectus luteo-, abdomen rubro tincto. Al $x$ maculis fasciisque albis nigrisque varix. Cauda rigida cunriformis: recrricıbus basi nigris, apicem rersus albidis maculis per paria nigris, intermediis 2 totis nigris, tectricibus uperioribus nigris. Pedes caruleo-cinerti.

5. major: niger: fronte sordide rufescentealba; occipitc coccineo; macula temporali, collari, scapılari- majoribus remigibus maculis parvis albis; crisso miniaceo.

$f$. occipite nigro.

Linn. S. N. 176. 17. Fn. 100. Retz. Fn. 55. Homm. Fn. sor. Sparrm. Sv. Orn. 8. h. Nilss. Orn. 52. Fn. 3. 13. 5. - Sv. Zoul.

S2. Stor II ackspik, Skngsskata, Vedknarr. - N. Stor Makhespet, sort- och hid. splinglet Hakkespat.

Hab. curn prioribus, sed frequeatissimus, manens et oта 4-6 ponens. Clamital: $g i c k, g i c k, g i c k$ ! - Longirudo ro poll. Rostrum cxruleo-fuscum, subias pallidius. -Iris albida. Inter maculas albas scspularis per paria abis. Subtus sordide rubrescente-alba. Cauda cuneata : rectricibus rigidis, intermediis 21.4 nigris inmaculatis; lateralıbus versus a picem sordido albis tasciis nigtis.

6. medius: albo-nigroque varius: fionte sordide ferruginescentc vertice coccineo, macula scapulari magna alba; subtus albidus: striis lateralibus nigris, crisso roseo.

f. panlo minor: fronte verticequc colore ninus clas'o.

Linn S. N. 176. 18. Fn. rox. Retz. Fn. 55. Hamm. In. 102 Sparrm. Sv. Orn. 7 L. Nilss. Orn. 5j. Fn. J. 15. 6. - Sv. Zool.

Sv. Mcllan Hackspik. - N. Flagspxk, Fleksparle.

Hab. cum pracedentibus minus frequens. Simillimus P. majori, sed megnitudine ininore longitudine tantum $8 \frac{1}{2}$ poll rostro cæruleo-nigricante ad bain piumbeo, fronte Cerruginescente, iride griseo-albs, vertice tolu el maris et femine coccineo, coloze subrus albido pectore luteo et abdomine rubro tincto crissoque obscure roseo, dislinclissime differt.

7. minor: nigre: fionte, macula pone oculos fascia collari fasciisquc in dorso alisque albis, vertice coccineo, tempntibus rubrescentilus, subtus striis lateralibus fuscis.

$f$. fronte verticeque sord de albis.

Linn. S. N. 177. 19. Fn 102. Retz. Fil 57. Sparm. Sr. Orn. 7. h. Hlamm. Fn. 103. Nilss. O11. 54. Fn. 3. 1\%. 7. - Sv. $200 \%$

Sv. Lillllacks pik. - $N$. Lille Trapikke.

$H a h$ in sylvis ubique etsi rarior. Viclus ejus ut pre. cedentium est. Ora a-7 albat virdescenia tiacta ponit Cuntinuo clamitat: $g i c k, g i c k$ ! - Characteribus iudicatıs, 

longitudine 6 poll., rostro viridescente-atro, iride fusco rufa, crissoyue fusco-maculato facilime cognoscitur.

-) pedibus tridactylis:

8. tridactylus: albo nigroque varius: vertice Havo, lineis longiludinalibus latere colli albis; subtus albus: striis maculisque latcralibus nigris.

$f$. vertice argenteo, nigro-striato.

Linr. S. N. 177.21 . Fn. 105. in Act. Holm. 1740. 2140 Retz. Fn. 58. Nilss. Oin. 55. Fn. 5. r9. 8. Sv. Zool. S2. 'Tretås Hackspik.

Hab. in pinetis sat rarus, primum Dalecarlize D. Linnaus. - Longitudo fere 9 poll. Rostrum caruleo-nigricans, basi albidum. Iris fusco-rufa. Frons et vertex flavi, occiput nigrum. Cervix alba. Latera capitis collique nig1a: linea una sujra nltera infra oculum alba. Dorsum nigrum antice albu.immixto. Remiges nigri: marginibus albo-maculatis, apice albo; tectricibus nigris. Crissum sordide-albuın: lineis uransrersis nigris. Rectrices 6 medize nigræ, sequentes $2-2$ nigrx apicum versus albx fasciis nifris. Pedes grisei.
Subclass. r. ACTIORNITHES, Ord. ז. THASSOPODAE, Nat. 19. II EMATOPUDIDES,

G. 56. HeMATOPUS *), Sv. Rödfos.

๘. ostralegus: ater, sublus dorso posticn, fascia alarum gulaque, basique caucla albis. Linn. S. N. 257. I. Fu. 192. Retz. Fu. 170. Hamm. Fn. 205. Nilss. Orn. 150. - Sv. Zuol.

Sv. Ostrons Rödfot, Strandshate, Strandskjura, Marspitt, Spiog, Albrok. $-N$. Rödfod, Kield, Tield, Stuandshade, Strandskiur, Ronkalv. - L. Sayan.

jun. colore atro adulti fuscescente striis fer. ruginescentibus; rostro aurantiaco ad apicem fusco, iride brumea, pedibusqque livicite-gliscis.

Hab. ubique in lilloribus lapidusis marinis, rarius aquarum dulcium; migrans autumno revenit inense Martio. Conchis conclileinglue, insectis vermbusque masinis rescitur. Nidulatur in pratis paludosis raro in littore, ova 2-5 olivaceo nigro-maculata ponens. Territus sonum guy y edir el clanose sun srepe aves alias fugere venaiorem monel, ipse vero vulneratus letum pritat nasanilo. - Longitudo 16 poll. Riosirum el palpebix clare aurantiaca. In coccillea. Pedes clare sanguiue.

-) Hab. gener. corpus minus compressum quain aliarum Thassopodarum: collo brevi; alis mediucribus remige l:o longissimo; cauda breviuscula: rectricibus 12; pedibus robustis: digitis tantum extimis meinbrana basi connexis. - N. gen. e vocis errecis cilfuce sanguis ef $\pi$ s $_{5}$ pes. 

Nat. 2o. CIIARIDRIIDES,

\section{G. 5\%. CHARADRIUS *), Sv. Pipare.}

-) pedibus mediocribus:

1. plusialis: supra verticem dorsum alas candumque migricans: maculis majoribus lue teis; laleribus capilis collo pectoreque cinercoliusculuteocine inaculatis, ceterosubtus albidus. Linn. S. X. 254. 7. Fn. 190. Retz. Fn. 168. Hamm. Fn. 2.94, 205. - Vilss. Orn. 145. Cli. apricarius. Sv.Zool. Sv. ll'gn pip.l'e, Ljungvipa, Ljungspole, Ly" 万รspole, Brockfầel, Marpytla, Aivarglim, Akrohöna, Heidlı̈ua. - N. AkerJoc, Ficld'ne, Lovetruel, Heyloc, Heiloe, Hookfigl, Myreloc, Reguspoe. - L. Huet, Huit, Hutti, Huttuh, Cizhiutzh.

(astuce) nigricans: maculis fulvis albidisque, fascia a fronte ad latera colli decurrente alba.

$f$. colore misus viridi: fascia a fronte ad lalura uique pectoris sordidiote corporeque sublus penuis plaribus albis immixtis.

Linn S. N. 25 6. Fu. 183. fietz. Fn. 107. Nilss. I. c. Ch apricsilus.

Hab. in lecionibus boreslibus xitate, ineridionalibus gregatu aulumuo; versus hienem enrgrat jeveniens menso Aprili. Progrediualar serie iriangulario Ora 5-5 virideoliracea

-) Hab. gen. statura pracedentis sed corpore ut et in generibus sequentibus compressiore: alis mediocriluss, remige 2:0 lungissimo; pedalius mediocribus t. longus, gracild, ds. digisis cuntumb extimis ad basin membrana counexis. - Nom, gener. gracum Augudoeós. olivacea, nigro-msculsta ferm. supra terram ponit el dies $2 n$ in wbat. Insecris vermibusque victitat. Silulat: ol $\mathrm{g}$ ik: - Longitudo maris $\mathbf{t r}$, fem $12 \frac{1}{2}$ poll. linstrum nirrofuscum. antice 13. rum. Iris lissa liemiges nigri, bas. a!bi: nrimores aciminati, lárere intariore ad medlunt albi. rachibus a medon rersus apicem albis; secundsrii obeu. sinses apicibus albidis. .tami 5. a langi colure dorsi Cated fusca: linais irav vame 8 ad sichos interruptic fulvis, in recirabis laterabus abidis. Pedes lusco-ci Darei.

2. morinelius: crines-fincus: manginibus

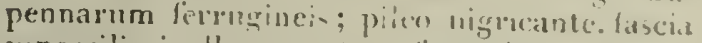

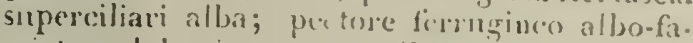

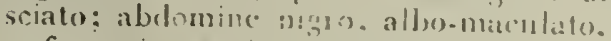

f. caplice intice masulis minulis striisque griseis; Inra nirral; sula albas: prectnre ferru. ginco, ablominefuce wigros immanculatis.

(anfummo) fuscus: nasıgimilus pembarun. rufeseculibr:x, dorso alisque fincis, froute al. bicante maculis parris fiscis, fascia superciliari sordide-allar pectoreque abiapte nigromarginalo.

Linn. S. N. 254. 5. F13. 188. Fiels. In. I66. Hamm. F11, 202. Nilsi Ors. $146 .-$ Su. Zool.

Sv. Mn rincrl'ipile, Pomerinsfigel. - $N$. Pehrloc, boldtixt. - C. Liahuil.

Jun. malginibus peillanıun capitis pallide rufis, fionte allidio finsco-masulata, lascia su. perciliar sordide rufiescente, fasciareuc pecto. rali vix ulla.

Linn. Fn, Su. ed r, n, 16n.

Mab. non infrequens in Lapponia, ubi nidificat. Migratorius regiones australioses quxrit mensıbus Aus. Sepr. rerenions Apr. Hajo. Vespere rivulos petit cluinilaro 
dyt, dyt! atque evolans: dry dyt dyc! Insectis vermibusque vescitur. - Longitudo maris $9 \frac{1}{8}$, fem. 10 poll. Fistrum nigrum. Iris lusca. Pectus liypochondidayue saturato-f-rrugınea (maris albo immixto). Remiges grise-fusci: margine interiore a basi pallide cinereo, prima rachi alba. liectrices fusco-cinerea versus apicem nigricantes, albo-teıninatæ; exteriores quoque margine exleriore albx. Pedes viride-cinerei.

") pedibus longioribus:

3. hiaticula: fusco-griseus: fronte, collare, linea alarum obliqua, corporeque subtus albis; collo nigro; remigibus plurinis medio longitudinaliter albis; pedibus luteis.

$f$. colore nigro magis grisco immixto et uigredine colli angustiore.

Linn S. N. 253. 1. Fn. 187. Recz. Fn. 165. Nilss. Orn. 147. $H a m m$. Fu. 201. - Rudb, av, pict. (Dc Geer.) 188. Hiaticula favipes. Sv. Zool.

Sv. Gapliparc, Strandpipare, Strandrulting, Grylle.

Var. B: albo-cinereo-fuscoque variegala: fascia frontali, collari corporeque subtus sur. dide-albis.

Hab. in litoribos sat frequens post medium Martii ubi ovs 45 pallide lutescenlia, punctis maculisquis parvis nggris in arena ponit, mense Octobri emigraus; vat. (3. in insula Sudermanuize Moorkön rarissinua $D$ Ekscröm. Insecta, larva rermesque priccipue aquatica cibun el prabent. Clamitat: jy, jy! et terrilus: $t y c k$ cyclit $t u l t$ ! - Longitudo $7 \frac{1}{6}$ poll. Kostruin aurantium apjee nigrum. Tris fusca: margine palpebraruun aurantusco. Tascia s!ujua frontein olbam, aliaque infra oculos postice latior nugre. Macula frontali per oculos, gula et jugulın superius aiba. hemigus nigro-fusci, latere interiore a basi albidı: jrimores $x-4$ raclii media alba, sequentes macula longitudnali racliali alba, qux macula sensim augetur ut pen-
A* 19.2I plane sunt albre; tectricibus secundariorum fuscis apicibus albis, unde lineola alba in ala complicara. Cauda subrotuma longitudine alarum: reclricibus I-r. aibis (interdum latere interiore macula fusca),2.5 griseis picem versus nigricantibus, ipso apice margineque exleriore albis, 4-5 basi griseis a picem rersus nigricantibus, apicibus albis 6-6. immaculatis extus nigricantibus.

4. fulvipes: fulvo-grisens: fascia frontali collare corporeque subtus albis; fascia pecto. rali nigra; alis immaculatis; pedibus clare carneis.

$f$. fascia frontali alba tantum maculifosmi, nigredine occipilali per oculos anertstiveri.

Rudb. av. pict. (Dc Geer.) 189 . J. - Nilss. O1n. 15. Cl. $_{\text {. }}$ minor. Sv. Zool.

S2. Gulfots Pipare.

jun; colore nigro pallidiore et fitsco-griseo obscuriore; margine pennarum supra rubrescentibus; basi rostri luirsente.

Hab. in litore lacuum et fluviorum, interdnu etjan marino, nigrans. Insectis vermibusque a juaticis viction nidulatur in asena et ova $3-5$ luteo-alba macuitis puncrisque irregularibus nigris et luscis ponut. Siblat: hirr, kirr! - Differt ab antecedente, cui similis, magnitudine minore, longitudine tantum $6 \frac{\frac{1}{x}}{4}$ poll. rosen 1010 fin adulcos nigro, fascia lrontali alba tantusn intor eculos, fascia supra vesticen per oculos ad latwód caputis nigra cum rosiro fasciola ab oculis nigra connecta, colnı sıpra magis griseo, lineola alarmm alba nul!a realihuscine clare cameis. Nomell specificum inventoris primi Culeb. D:i Rudbick Fil. lıad mutaudus est.

5. elexendrinus: fusco-griseus: fronle collare, collo antico corporeçue sublus niveis; rectricibus 2 utrinque laterabus atbis; peliJus, uigris. 

f. abspue macular vertu an angulari, fis:? tilltum angus!a nigra.

Linn S. X.253. 2 - Ascanius in Act. Nidros rol 5. 153. t 5. Ch. Alexandrinus. J. Frinnich Or nithnlogia borealis. app. p. 77. - Nilss. Orn. 199. Ch. allutirons. Sv. Zool.

Sr. Alexaludriusk Pipinde.

Hab. in littoribus almosis inasis, procipue ad oslia Auvinum Nusvegix D. eiscanius (minine Crocethia Cu-

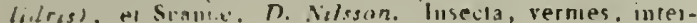

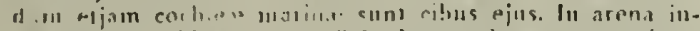

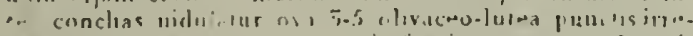
- Arilus plus mullise magnis luscis pougens. - Longida vit. poll. linsirum nigrum. Iris fusca. Ceterio e

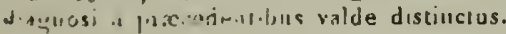

(i. ,8. CR(jeTHIA *), Sv. Strandlyipare.

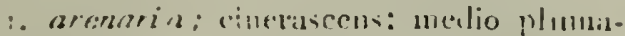

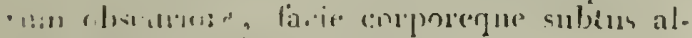

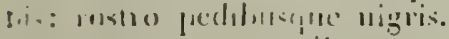

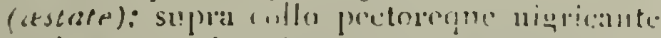

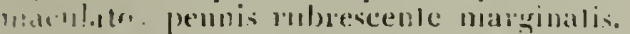

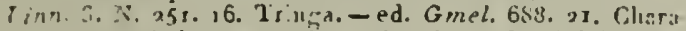

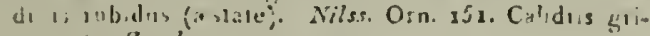
sit. $\therefore 2000$.

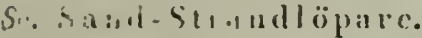

jun. capilfe, dorso, tectricibus alsmum ui-

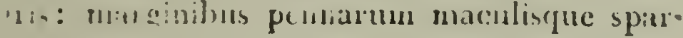

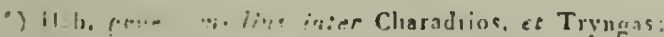

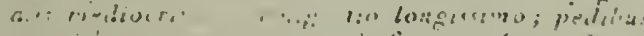

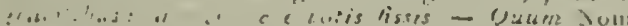

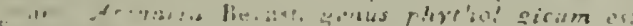

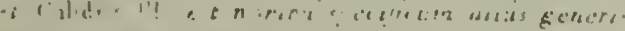

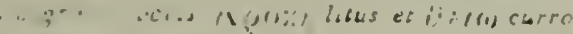

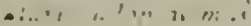

siv lutescentibus; lasciola inter neulos of in.

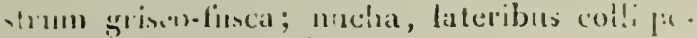
ctorisque griso'sceutibits.

Linn S. N. 255 9 Cluardrius calidris.

Hab. in litoribus arenosis marinis passim. Inseativ variis marinis victilal. Nidu in suum redificat el ov, poult in regunubus hyperboreis: Clamilat pite! Funigut allIumnce redux vere. - Longitudo $9-7 \frac{1}{4}$ joll!. Iris brumus, Latera culli cineroscentia medio plumarum obsurine. Mlargn alarum, remigibusque primoribus nigrio, rachibus albis, secundariis a bası ad mediun alts.s, deandw misris apise albo-marginatis, tecurcihus albo marginatis. Kactli ces 12, qunarum intermedie 2 longiores nigricanles, cascor grigex marginibus albidis.

\section{Ord. 2. NEOPODAE,}

\section{Trib. 1. PROMACRORHAMPHAE,}

- Nat. 21. VANELLIDES,

G. jg. VANellus ${ }^{*}$ ), Sv. Vipa.

-) capile haud cristato:

1. helveticus: nigro-fuscus: maculis viride. luteis sparsis penuiviue albo-marginatis; subtus albus maculis lateribus colli, pectoreque fuscis cinereisque; cauda alba fascia maculis. yue niglis.

(restate): nighicans: maculis trausversis al.

-) Hab. gren. Charadrio affunis, alis acuminatis; re migibus vel tantum 1:0 vel 4:0 ef 5:0 longissimas; perdibus gracilibus: tibiis digito medio longiorihue. digitis externis articulo 1:o connexis, postico brew elevaro. - Nom. grener. Bellonii wst. N. latinus ite bistum. 
his, linca fiustali per lalera colli ad alas us. que nived; ublus niger, erisso caltuaque ni. Erro-fanciala albis.

Litin. S. N. 250. 12. Tringa. - Nilss. Orn. 174. V. me. lancgaster. Sv. Zool.

Sw. Sich weitzer Vipa,

jun. sinulis adulto licme sed supra colore magis grisco albo variegato, sublus fionte, supereiliis lateribusque pectoris grisen-macnlatis; fasciis cumcle grise is; pedilus virescentegriseis.

Linn. S. N. 252. 25. Fn. 186. Tringa squararola.

Hab. in horibus mariuis arclicis. D. Leche. Inseclis vemibusque tetrestribus et aquaticis victlat. Nidifirat in arena, ova 4 pallide olivacea naculis nizris poneus. - Cum Charadrio pluviali. cui un habilu astivali simiis, nou confundendus; diftent prumo inruilu digito poslicn. Longitudo 10 -11 poll. Rostrum nigrum. Inis tusca. linmex sis longissinns. Pedes obscure-prisni reticulari: diguto postico brevissimo. Celero per diagnosin suficientos disinclus.

$$
\text { -) rapile cristato: }
$$

2. crisiatus: atro-viridis, pur pureo-lucens; -apite, collo subtus, pecture candagre media extisua nigris; orbila oculorum, collo supral, nopygio, corporeque subtus albis.

f. crista breviore, gula juguloque allo-maculatis, nigrediuc pecturis minore el minus ubicura.

Linn. 5. N. 238. 2. F11. 176. Retz. Fn. Iis. Hizmm. Fil. 880. Tringa. - Nilss. $173 .-$ Si. Zuol.

S2. Tof's $\mathrm{ipa,} \mathrm{loppvipa,} \mathrm{Vipa.-N.} \mathrm{Yibe,}$ líbils.

Mal\%. ub que in pratis paludosis Scandinavire australi; abit autumuo redieus ad finem mensis Febr. rel incunte
Miruo. Insecla vasia, vermes ef testacca cibum el frabent. Nidulatur in caspritus pratorum inter sraminal. scirpos, ova 3-4 olivacea muculis plus ininusve magnis niglis in extremitale majore confluentubus ponens quxe dies 16 incubat. Clamital: tjyvitz! - Longlludo $12 \frac{?}{8}$ poll. hostruin nigrum. Iris brunuescens. Reniges 1,0 et 5.0 longissimi. Rectrice extima alba: lectricibus inferi. oribus rufis. Pedes obscure-rubri: unguibus vigris. $\mathrm{Ce}$ tero per characieres indicatos beue cogititus.

\section{Nat. 22. SCOLOPACIDES,}

Div. 1. diginis ad basin usque fissis.

G. 6o. STREPSILAS *), Sv. Stenvält.

1. incerpres: niger: capite colloque supla, macula inter oculos et rostrum, grula, corpore sublus, albis; scapularibus tectricibusque ala. lum ferrugineis rivgro-maculatis.

$f$. colore sordidiore el nigredinc paulo pal. lidiore.

Linn. S. N. 248 , ห. Fn. I78. It. Goltl. p. 2 17. Retz. Fu. 150. Hamm. Fin. 190. 'Iring rinella collıris. Sv. Zool.

Sv. TolkStenväl. $-N$. Kulterutte, Vegdetite.

annocinus: Iocis nigris in collo pectorecule pennis nigris auguste albo-marginatis, fronte

-) Hab. gener. precedenti proximum imprimis se. ctioni prinar; alis acuminatis: remige t:o longissimo; pedibus mediocribus: femoribus supra genua parum nudis, libiis longuludine digiti medii. -

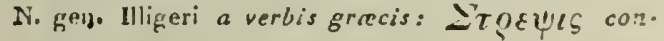
zersio, -1as lapis, conservavimus, quia antiguib. quam Morinella Meyeri, quod nomen us specirigu Generis Claradrii ineptum esc. 
alba punctis ngris, vertice muchagne brum. neis firsco-muculatis, doron, scapularibus et tectricibus alatuns migris rubucscente-margiuatis, fectricibus latcralibus nacula nigra, ectero ut in idnlto.

adluc junior: absupe colure nigro l. ferruntlleo; capite nuchaque brunneo-griseis lincis fuscis; penuis latrablus colli fuscis apice a!bidis; dorso, scapularilus lcetricibusque alic. rum fuscis latc futessente-marginalis, fascia candali fusca; pedibus lulescente-rubris.

Linn. S. N 24.?. 6. Tringa moriuella.

Hab. in litnribus mon inlreguens. Migral autumno rvaniens tenpore veruali. Victitat inseclis, varmibus testacesilue marınis, yua lapides rertendo colligit. In airia nudulatur et ova $3-4$ cineredo-viritia maculis luscis po:11. Clamitar: llyo! - 1ouguralo 80 poll. Rosumm nigrum (juniorabus basi fuscrscems). Tris lusca (junioribus hrunuea). Pennce scapulares fere lobx, tecricesque margine ferruginute Holniges milicalles latere iuteringe primores ad basin el inlomi line luti albi. Cands a'qualis: lascia las fusca ad apicem albs. P'edes aurannisci: un; $; 1$ ibus nigris.

Div. 2. Dipisis ad basin connectis:

G. 61. PISOBIA *), Si. Fukisnäppa.

1. minulu: niggofunca: jumus grisco-muar-

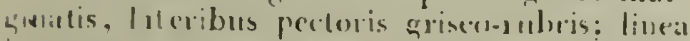

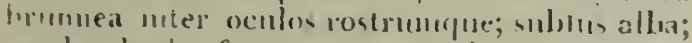

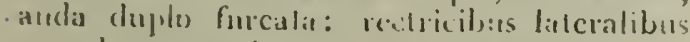

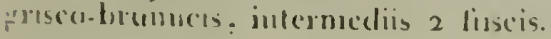

\footnotetext{
11..t. geutr. Tryaggarum; recericilus : intermedus

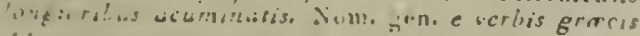

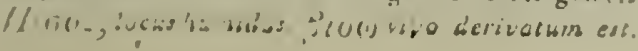

(cestate): pileo niturio, maculiv lulluis; lateribus colli pectorisque clace rubrescentilus: dorso, scapularibus tectucichnsque alaumu, ieclricibusque 2. intermediis atric.

Leisler Nachit, zu Berhateins Nammer Deulscill. He[t. I. p. 74. Art. 2o. Tringa. - Sv. Zoob.

So. Lill Fuhtsuäp pa.

jun. nigro-fusca: marginibus pennaruru ferrugineis et albis rachibus albis; subta aliba Retz. Fn. 164. Tringa pusilla. Nilss Ora. 182. Triuga.

Hab. in lilloribus maritimis regionum seplenttwona!um mınus frequens, $D$. Retzius. Redur vere, abit antmmno. Insecris vermubinsque parvis vescitur. Nidulatur in lucis humidis ova 4 pyriformia pallidelutea, maculis cinereis plusque minusve trunneis ponu. Vorem sibilautens emis iit. - Longitudo $5 \frac{t}{4}$ noll. Mosırmm nierum. Iris .. .. eupl:Tcilia, jugulum, medium pectoris, abdomen, crisimn, le clricesque laterales caudx abba. Raches remigum alba Reclrices albo-marginati. Pedes nigri: Jbiis longitudinc ro lin.

2. Temminckii: nigro-fusca: peunis lumuneo marginatis; collo auticn pectoreyuc rublue stente griscis; jugulo corporergue subtus albis: cauda suhcuneata: rectricibus lal('raliluss allois

(astate): nigro-fusca: margine peumarut: late ferminico, froule, collo auticon percoreque rubrescente-grise is lineolis nigris; jugulo corporeyte subtus rectucibusque lituralibus

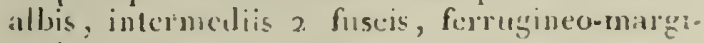
natis.

Leisl. Nachir. 2. Rechst Ẍat. Deut. \$. 65. 9. 70.5 Nilss. Oin. 181. Trings. - Sv. Zoob.

Sv. Temm inchs Fuktsü̈pa.

jun. ngro.grisea: nucha pallidiore, ucou

lutescontc-anarginatls; apicilus scapulus. H 
fasciola nigra; laterihn; colli pectoreque gri. seis rubrescente tinctis, celero subtus alba; rectricibus extimis exceptis apice rubrescentibus; pedibus viridescente-brumeis.

Hab. dilora mariua in regionibus arclicis, ubi uidificat et sese propugal aslale, D. Nilsson; migral, auIusno, redux mense majo. Pracedenti valde similis et longiludine tanlum 5 poll, 6 lin. sed differt colora magis infiscalo, lineola inter oculos rosirumque deficiente, rectricibus 4 inlermediis griseo-brunneis. celeris albidis, extimis slbis, rosiro pedibusinge fuscis.

\section{G. 62. TRYNGA *), Sע. Snäppling. -) recuricibus 2 nuediis longioribus.}

s. cinclus: ciucreo-fusca: rachibus obscu. rioribus; cervice pallidiore; subtus alba collo anlice cincrascente striis minutis; rectricibus intermediis 2 longinribus acuminatis.

Linn. S. N. 25r. 18. lietz. Fn 159. Nilss. Orn. 180. t. 10. - Sv. 7 ool..

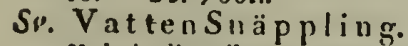

Hab. in littoribus septentrionalibus, ubi nidificat, sed visil lilora australioria preserlim balıhica autumno liemeque. Secandum $D$. Nilsson hanc speciem revera a Tr. alpina L. (Tr.variabili) quamvis ei similem, esse diversam el minime varietalem ejus hibernalen, persuasi sumus. Langiludu 7 poll. Rosirum nigrum. Iris nigro-brunnea. Frons lateraque capilis abida fasciola inier rostrum et oculos fuscescenie supra quam stria albida. Remiges ni-

7) Il.ab. Kau. fere generuin pracedentium'sed rostro bene distinctus. Alre mediocres: remige 110 longissimo; pedes graciles, breves: digith al batin usque jissis. - Nomea geller. gracum Aristorelis I'purres. gro-fusci, rachibns albis, extimi 4-5 latere inleriore medio cinerei apicibus obscurios ibus, sequeules maryine exteriore medio albi, albedine crescente imeriorum usque ad marginem Iolum album, macula tanium ad apicent fusca. Tectrices alarum cinereo-fuscre marginibus dilutioribus el rachibus obscurioribus, apicibus iuteriorum vero albis, unde siria alba in alis complicalis. Cauda rectricibus 2 iuternedis exceptis aqualis, quarum latere exteriore rectricum cinereo, inleriore obscure.fissco; rectrices celeræ albo-fuscescentes rachibus albis. Pedes nigrobrunnei.

2. alpina: ferrugisen: capite colloquc pallidioribus; subtus albida: striis maculisque nigris, in dorso, alis pectoreque inferiore na. joribus subtransversis; rectricibus 2 interme. dis longioribus acuminatis.

(aucumno): vertice rufo nigro-maculato, cervice cinerea fusco-striata; scapularibus dorsoque cinereis, peunis ferrugineis nigromaculatis immixtis; pectore sordidiore, cetero ut iı vel'e.

Linn. S. N. 249. 11. Fn. 181. Retz. Fn. 155. Nilss. Oru. 178. - sv. Zool.

Sv. Fjäll $S_{\text {ü }}$ ppling.

Hab. in regionibus arclicis, eljam in Lapponia, $D$ : Adlerheim, locis paludosis. Migral aulumno el revenit primo vere. Nidulalur inter gramina el ova 4 pallide-viridia maculis in extremilate majore brunneis nigrisque sparsis ponit. - Longiludo $7 \frac{3}{4}$ poll. Fosırum 11 grim. Iris fusca. Facies albida sIriis minulis punclisque fuscis sparsis. Collum aibum naculis parvis numerusis unris. Abdomen crissum et lectrices inferiores laleralesque superiorum alba immaculala. Remigos nigro-fusci, latere inleriore a basi cinerascentibus, 5.8 margine exteriore a! bi, secundarii lalere inleriore a basi margineque exteriore apicis albi, intumi 5 elonyati, acuminali, cinereofusci. Uropygium et tectrices superiores caudac modic 
nigro-fesca, imsuaculate. Rocirices $1-5$ equales albo-foiscescentes, 6-6 obscure-foscx. Pedes nigro-fusci.

3. maritima: nigricans collo antico et la. terali magis cinerascente, orbita oculorum macula ad rostrum gulaque albida; margini. bus penuarum albis 1 . cinereis.

(astate): nigra: marginibus pennarum late albis et lateraliter anguste rubris; subtus cinereo-alba: maculis colli antici, pectoris abdominisque lanceulatis, laterim colli ovalibus, tectricum caudx Iongitudinalibus nigris; me. dio abdominis albo.

Linn. S. N. ed. Gmel. 678. 36. Nilss. Orn. 177. Stroem in Act. Nidr. 3. 440. to 7. Pontopp. Nal. hisl. 2. 318. Brünn. Orn. 182. - Sv. Zool.

Sv. Ha fs $S$ ü äp ling. -- $N$. Fixremuus, Fjæ- replyt, Fjerekurv, Fiörepist.

jun. supra cum pennis secundariis alarun intermediisque candie pallide-nigra, nuarginibus pennarun clare rubris; tectricibus apice late albo-marginatis; collo antice lateribusque striato, peunis cinereo-marginatis; lateribus corporis abrlomimeque longitudinaliter macu. latis, rostro pedibusque clare-luteis.

Retz. Fn. 15r. Tringa siriata.

Hab. in lisoribus marinis, precipue tamen Norvegiae soptentrionalis el Lapponix, ubi nidificat ova 4.6 pallida maculis grisescenjibus ponens. Sonat evolaus $p l y e, p l y l$ ! - Lollgiludo fere 8 poll. Kosiruni ni.̈rum, basi uibiescens. Iris clare-umbrina. Tecuires alurun nigricantes cinereomarginalis. Abdomen in medio album, sed lalera corporis maculis magnis obscure-cinereis. Tecırices inferiores caudze maculis larceolatis nigricanubus. Uropygium reciricesque, injermedize alrs, rectricibus ceteris cinereis albo-marginatis. Pedes ochracei : unguibus nigris.

") canda xquali.

4. islandica: cinerea: marginibus pennarum albicantibus; subtus allba: pectore cinerascente, striolis fasciolisque semilunaribus brumnescentibus; cauda æquali.

(astate): nigricalıs: marginibus pennarum ferrugineis; subtus pectoreque fermgineis superme, abdomine albo fermginco-nigroque-maculato; cauda obs:nre cinerea: rectricibus albomarginatis tectricibus albis: fasciolis semilunaribus nigris maculisque ferugincis.

Linn. S. N. ad finem Parro 2 Tom. x. 19-20.(Addenda): restre. Retz. Fn. 167. Hainm. Fin 194. - (restat.) 16x. Tr, grisea. 16a. Tr. cinmrea them.1. Hamm. Fn. 192. Nilss. Oin $\$ 76$. Tr. ferruginea Sv. Zool.

Sv. Islands $S_{u}$ äp pling. - N. Islandsk Tite. jun. colore cinereo obscuriore, maruinibus pennarum primun nigris, deinde albis; capite cinerco, maculis longitudinalibus brunneis; fasciola inter oculos rostrumque brumea; pectore rubrescente-griseo tincto; rostro viride. scente-cinereo pedibısque viridescentc-luteis.

Hab. in paludosis hyierboreis seslate ubi nidifical; migrans autumno el redux primo vere, rermibus insectisque aquaticis, raro testaceis vicuilans. - Longitudo lere so poll. Rostruin viridescente nigrum. Iris fusca. Juguluin et abdomen alba. Frons, supercilia, latera et anterior pars colli, pertils el liypochondria ba, striis fasciolisque rinereo-fuscis. Tecirices alarum cinerex mas grmbus albidis el medio fuscescentes. Urnpygium el lecirices supesiores caudx olba maculis iransversis nigris; reclrices ciaerexe albo-marginatz. Pedes viriciesceule-nigti. 

G. 63. MACHETES *), S*. Brushane.

1. pugruax: $m$. varicolor: rostro brunne. secnte; facie plunosia; collari e pcnnis mediocribus; cauda rotundata: rectricibus 2 intcrmediis maculis 1 . fasciis nigris, latcralibus 3 unicoloribus apicc albo-marginatis.

(restace): in. rostro liteo-aurantiaco; facie papillis lutcscentibus 1. 1ubrescentibus; collari e pennis longioribus a corpore discoloribus.

f. minor: colore cinerco pallidiore absque collari; rostro nigro; pedibus (assate lutcsecutibus l. viridescentibus,) /Lieme obscurioribus.

Linn S. N. 247. I. Fn. 175 . Retz. Fn. X47. Hamm. Fn. 184. - Nilss. Orus. 172. Tolanus pugnax. Sv. Zoo:.

Sv. StridsBrushane. - N. Brushane, Bruskop.

Hab. gregatim in pratis humidis et paludosis restate, raro in lisore marino; emigrans autumino revenit primo vere. Vermibus inseclisque ayuaticis vescitur. Nidulans inter gramina, fern. ova $4-5$ acutiuscula, griseo-alba, rufomaculala ponit, et 16 dies incubat. Quum in venerens runnl mares collaribus ereclis pugnas sape cruentas suscipunt, Sub emigtatione sonum edunt: $g i c k, g a c k !-$ I.ongiludu m. 32, fem. 9.9 poll. Iris lusca. Color ita raciabili, ut vix unquam 2 similes inrentuatuli characteres indicali lantum constantes et sufficientes sunt.

-) Ilab. gren. medius inter Tryngam et Caritem, ad roitruin prioibus, ad statiram posterioribus similis; pedubus longis: digits margine setratis, 2 externis membrana ad t:an articulationem unitis. - N.genrar. egraco: Mlül,Tis, pugnator. Hane avern jain ad l'ringas, jam ad Tolanos ab auctoribus durtwon, nou sine jure, ut proprium Genus, cum Celeb. D. Cuvier judicavimus.

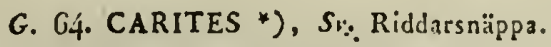

-) Semoribus fere lotis nudis.

s. fuscus **): cincreo-griseus: rachibus fasciolaque a rostro ad oculos nigrescentibus; subtus albus; tectricibus candx superioịibus rectricibusque fusco-alboque-fisciatis ; ' basi mandibula iuferioz is rubresccnte.

(astate): nigricans: pennarum margine albicante l. albo-maculato; subtus nbscure-grie sea; uropygio crissoque albis; cauda obscuregriseus interrupte albo.fasciata; pedibus rubrcsccnte-brunneis; basi rostri subtus rubra.

Linn. S. N. 243. 5. Scolopsx - ed. Gmel. 66g. 46. Scolop. curonica, -668 . 45. Scolop. cenlabrigensis. 67). 26. Tringa atra. Tengm. in act. Holm 1783. 48. Retz. Fn. 158. Scolop. - Nilss. Orn. 167. Totanus Svi Zool.

Sv. Brun Riddarsnäppa. - N. Skrilkixld, Quikketield, Squapkixld, Stilk, Solskiarstöd, Laxctile, Laxtitting. - $L$. Buvadak. jun. colore supra olivaceo tincto: nuarerini. bus pennarum dursalium lateralibus albis;

-) Hab. gener. pracedenti approximat; alis mediocribus; remige 1:0 longissimo; pedilus longis, gracilibus, femuribus plus ninusve nudis, cligitis 2 externis membrana basa!i unitis. - N. sen. e graco voce: Kapltris, eques; gallicum Chevalier; Totauus auctorum recentiorum nomcn est nec labinum nec grecum, unde rejiciendum.

") ATinime tamen Tringa fusca Iann. S. N. 252.22 , qua Senesalum inhabitat et differt colore immu. culato, rostro pedibusque fuscis. 



\section{1 任}

pennis tectri.mm alarum et scapularibns ma. culis marrinalibıs parvis triangularibus albis; subius albid:us maculis variformibus cinereogriseis; pedibus rublesceule-aurantiacis.

- Linz S N. ed. Gmel. 655. 12. Fn. 167? Scolopax totanus. - Hab in regionibus arclicis ad ripas lacuim, fuviorun $e f$ in locis paludosis. Emigrat alltumnn. Testncea, rarius insecla verniesque cibum ei prebent. Clamirat volitans: cait, tait! lerrills: gätsch, gätsch! et sedens gick, gick! "t $g a c k$ ! - Longitudo $1 \mathrm{x}-12$ poll. Rostrum nigrum: basi nandibula inferioris rubrescente. Jris obscurefusca. Uropygium album Hyonchondrix albocinerer. Genxe collininque antice lateribusque alba cinereo-lincla. Pedes clare-rubri.

3. calirlris: rineren-fiscus: inedio peunarunt Inngitudiualiter obsemrinie; nedio rostri basali rubro; subtus griseo-albus: striolis fuscis; abdomine mopygioque albis; cauda albonigroque-flexunce-fisciata.

(cesiace): supra colore nlivaceo-tincto: medio pentarmun capitis collique longitudinaliter nigri, pennis dorsi tectricmuyue alintum nigro. undulatis; subtus albus: striolis nigro-fuscis; rectricibns, f intermediis cincrascentibus exce. ptis, albo-nigroque-radiatis apice albis.

Linn. S. N. 145. 17. Sccilopax. - 248. 3. Fu. 177. Tringa gatubells. Nilss. Ora. 168. Tolanus. - Sv Zoot.

Sv. siknkiz li iddalsuäp pa, Rödbenil.

jun. bani rostai pedibusque sordide luteis; capite desorgle litsis: marginibus pellnarum

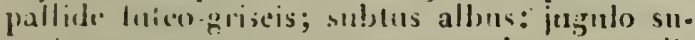
periorege frectore curcrasceutubus maculis parvis lungitudinalibus fuscis.

Lenn. S. N. 263. 5. I ring siriala.

Hab.
Hab. vere in pratis paludosis, astate in paludibus et littoribus maritinis Svecie Norvegixque ad regionen lapponicam belulinam usque non infrequens; nigrans aulumuo revenit mense dprili. Inseclis non coleoptratis. vermibus, et conchyliis parris rictilat, qux in aqua vodens colligit. In pralis inter gramina nidificar, ova 4 viridescenie-Iutea maculis fuscis, in extrenitale majore confuentihus ponit et dies 14 incubat. Clamore lamentabili ab volatu circum nidum, collectori ovorum id prodit Longitudo Ir poll. Rostrum nigrum, basi subtus ru. brum: caput, nucha, dorsum superius, scapulares et tectrices alaruin fusco-cinerea, rachi pennarum striisque traus. rersis nigris: linea a mandibula superiore ad oculos alba. Uropyrium album. Lalera capitis et subtus alba maculis parvis longitudinalibus medio pennarum nigris, in abdomine tectricibusque eandx inferioribus obliquis. Rectrices albx nigro-fasciatre a picibus albis; 4 internedix cinerex. Pedes cinnabarini.

3. stagnatilis *): (hiemre) cinereus: mar. ginibus penuarum albicantibus; subtus albus: maculis in pectore transversis brunneis; rectricibus exterioribus 2 extus linea flexuose fu. sca; pedibus viridescentibus.

(asiale) supra cinerells, rostro nigro; rachibus pennarum capitis collique nigris, ceteris pennis rufescente-griseis maculis transversis nigris; in scapularibus et tectricibus alarum diagonalibus; rectricibus intermedis cinereis nigro-fasciatis.

Bechstein Naturgeschichte Deutschland, vol. 4. p. 26r. Tolanus. -

-) Hoc nomen specificum Cel.Bechsteinii praculimus: quia Tolanus, ut ab itahana lingua sumbum, ineplum est nomen. 
Jun. 3mpra fuscescens: marginibus peri!rarum lutescentibus, facie lateribusyne ianpitis punctis fuscis; pedibus cinereo-virideiceulibus. Zinn S. N. 245. 12 Scolopox toltnus. Sv. Zool.

Sv. Träsk Kiddarsnäpra.

Hab. ad ripas furiorum in regionibus arclicss Svecite Norregixque, emigrat sereno autumus per teras wienrales; insectis aqunticis rermibusune victilsas. - Lonairudo circiler 9 poll. Rosirum ciueren niguum. Ins brumes. Superciliz, facıes, mediunt deini et sublus alija: nac:alis in laleribus colli el in persere transversis biungeis: caput supra, dorsum superius, scapilares et tertrices ald. rum majores cinerea: marginibus penuarum alb.cauribus: tecrices minores cum semiglbus exlerioribus ugrnacineseis. Cauda albo rectricibus intermeilies fusco-lasriatis, exlerioribus a extus linea flexuosa longitudiunli Jusca. Pedes olivaceo-virides.

4. ochropus: (hieme) fuscus viridi-nitens punctis pallide testaceis adspersis; superciliis subtusque albis; basi cauda reotricibisplute exterioribusitutis albis, pedibus viridescentc.. cincercis.

(ascace) colore superne obscuriore et massis viride-nitente: punctis numerosioribı!s naculis in collo antico-gultas tormantibus.

jun. colore pallidiore punctisque sparsius lutescentibus, nucha cinerancente, labreribns pectoris colore dorsi maculis albis; conto at 1. tico unedioque pectoris maculin lanseiforsnibus fuscis; basi caude alba angustione et ridscits linlioribus.

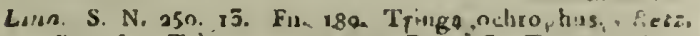
Fn. 154. Triogse: - Hamin, Fn. igi Thinge ochicphus. Nolis. O1w 169 Trianus. : S S. Zoot

sy. GulfotsRiddarsuapu, Eu,kel Bećsio. sin. - N. Jordyeed, Skoddefugl, Skoddeföll, Rosgork, l'lougmand.

llab. ad marçiues lacuum, raro maris, et ad paludes sal Irequels; enigrans autumno, mensis ad finen Aprilis revellit. Insectisyue non elmulheratis, precipue dipleris, cun vernibus minntis vescirur; nidulalur in arens vel inter granian ia rifis at ibı poilens ova $5-5$ albescente-viridia maculis fuscis, quie 24 dies incubat. Criat perpetuo $g y s$, gys, gys! currens sgilier ill aquo non profunda. - Lencitudo circiler $\$ \frac{1}{4}$ poll. Rostrum nigrum basi subtis virescerie. Iris tusca Fascia alba el filsca inler rostron el oculos. Siurra olivaceo-fuscus viridi-nilers marginibus pennarum dosi siapularium el leciricum alornm punctis minutis et colli strivis foncifudinalibus albis. Trectrices caudre et subrus albi, aed cullo antico pecroreque superiore marulis uumerosissinis Inngindinalibus fuscis. Cutuda alba fasciis $5-\mathfrak{q}$ latis fuscis cxtus dimumendis; rectricibus 2 mediis longionibus, aculis. Pedes viridescenle-cintrei.

5. glareola: (tieme) supura fuscus: maculis parvis allis; supereilicis, pectore, abdomi-

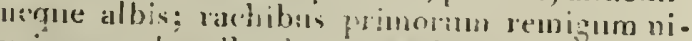

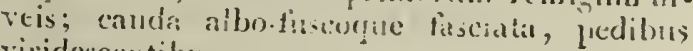
viridescentibus.

(astate) covice nuclacite albicante fuscoque striatis; genis, latcribus anticora: colli, superiore pectore albis, maculis Inrciondinalibus fuscis; peunis don'si unacula in.dio fiusen, lalese utroyne maculis 2 firvis albis, scapula. rimnçue nigro-fisciatis.

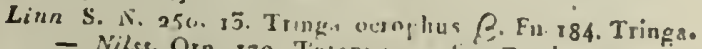

- Nilss. Orn. 170. Totanus. - Sv Zool.

Jun. colore fusco maculis parvis infescen. tibus unnerrisis; collo autico pectoreque superiore ciscerco-undulatis maculis irregularibus 
fuscis̄, basi rostri pedibusque lutescentc-viridibus; rectricibus irregulariter striatis.

- Linn. S. N. 2. ed. Gmel. I.677. v. R. Fun. r85. Tringa littorea. Homm. Fn. i85. - Su. Zool.

Sv. Grus Riddarsuä p pa. - N. (jun.) Skode. fole, Plongmand, Klövmand, Taagchest.

Hab. paludibus sylvalicis tolius Scandinavize sed minus frequens, insectis vermibusque victitans. Nidificat pre.cipue inira circulı m arcticum in paludibus ora 4 virescentia maculis fuscis ponens. Crial: $g i v, g i v$ ! et voce vibrante tiftirle. tiftirle! - Longiludo circa 7 poll. Rostrum nigrum, basi sublus virescens. Iris fusca. Listea inter rostrun et oculos angusta; lora, vertex, dorsum, scapulares et tectrices alarum fusca : maculis in margine pennaruin 3 parris rubrescente-albis; maculis alarum numerosioribus. Nuclsa, genx, collum anticusa, et peclus alba striolis longitudinalibus et transversis fuscis. Supercilia, jufulum, abdomeu, crisstin el uropygium alba. Remiges secundarii albo cincti. Tectrices caude superiores longiores maculis apice fuscis. Rectrices albo fuscuque-alternalim fasciatz, exterioribus $2-3$ margine interns albis. Pedes viridescentes.

6. hypoleucus *): nlivacco.fuscus: rachi. bus striisque trausversis fleverois nigro-fuscin; subtus albus: lateribus colli pectorisque lineolis Euscis; remigibus latere interiore macula magua alba.

Linn. S. N. 250, 14. Fn, 182. Retz. Fn. 157, Hamm. Fn。 188. Tringa. - Nilss. Orn, 171. Tolanus. - Sv. Zool. Sv. IIvitbuks Riddarsnäppa, Straud-

\footnotetext{
- Hanc speciem Tringam canutum Lina. Fn. 18j.esse, D. Nilsson opinotur, nobis haud placentibus, quunt descriptio Tringa ranuti in systemate ac Founo Linn. valde conveniens est, et hac sine dubio cum Tryoga islandica nostra eodem est.
}

sittare. - N. Lille Bec casin, Myrsueppe, Sitrandsneppe.

Hab. ad aquas dulces uhiçue sat frequens, raro aj salsas Insecta non eleutherata vermesque cibun ei prebent; nidum in litoris klaminosis adificat, ova $4-5$ lutescentia punctis macu isque $f_{u}$ is at cinereis, in extremu. tate majore majoribus, adipeasa ponens et it dies incu. bans. Veniens mente A.rli emigrat glegalı mense $O$. clobri; criarlunc perpelus hidydy $d y d y d i$ ! vihralıne Aluisda, solus evolitat ill aëre sono higög o $g \dot{o} ! \&$ \& 11 titat caudam modo Motacilloe. - Longuludo 7 poll. et ultra. Rosirum cinerem, apice nigrum. Iris lissca. Supra fuscus: olivacen-niteus rachibus nigris, pennis dorsi alarumque lineis hóxuosis transversis nigrn-fuscis. Stria super orulos a rosirn albi, sub qua alis fisca. Subtus albus late: ribus colli pectorisque longitudinaliter fusco-striolatus. Remiges nigro-fusri $1-\mathbf{I}$ cum intimis immaculatre, 2-10 markine interiore medin $\mathrm{macula}$ alba, 11-12 unedin apice. que a'bis. Canda cunealu-rotundata: rectricibus 4 medijs colgre dorsi transverse fusco-strialis, ceteris allon nigroque-fasciatis, apicibus albis. Pedes virescente-ciserei.

\section{G. 65. SCOLOPAX ${ }^{*}$ ), Sv. Sräppa.}

") digitis liberis.

t) femoribus fere ad genu plumosis:

1. rusticola: nigro-rufo-luteo-griseoque transversim variegata: occipite nigro fasciis transversis ferrugineis; cauda nigra: apice supra cinereo subtus albo.

-) Hab. gen. alce mediocres: remige 1:o poulo bre viore $l$. longiludine secundi longissimi. Pedes mediocres, graciles: femoribus fere nd genu usque plumosis vel apice paulo nudis, digitis plerumque fissis, raro externo medioque membrana unitis,

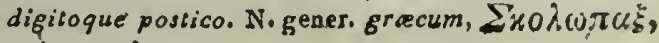
ovis quadam. 

f. major: colore nbe riore; maculis utime. rosis tectricmu alajur? alisis.

Linn. S. N. 245 6. Fn. 170. Retz, Fn. 159. $11 a \mathrm{~mm}$. Fn。 180. Nilss. Orn. 183. - Sv. Znol.

So. Land $S$ ä ppa, Morkulla. - N. Blomrokke, Rutte, Krogquist.

Var. $\beta$ : albida : rufescenti supra maculata, subtıs undulata.

Nilss. I. c.

Hab. ubique in syivis naludnsis, sat vulgaris, var. 3 ramen Uplandix, D. Halo de Paykuil, Iuissinit. Migrat sero autumno, redux primn pore, sceje minse Martio. Yidificat in graninos.s sub linficibus, ors 5 , rordide lutea: maculis parvis palide brunneis pounus. Insecla varia et vermes sunl ribus ejus; recla linea volicat somens: pits pits; sono fólem eljam pellicut. - Lunguludo 33-13t poll. Rosirnm rallide incarnatun! apice nigrum. Iris fusca. Supra rulo-lilso-grisenque-valiegata: nifculis mágnis 'nigritis ;ubtus lutescente rufa unduls rigrn-fuscis. Gula alba. Remiges fusci marginibus pimuruin rofo-maculteds, secundariorun rulo-fasciatis. Cauda roinndara. Pedes lividi: unguibus fuscis, postico brevissinıo.

tt) femoribus inferne nudis:

2. major *): vertice nigro: tænia pallide ferruginea; 'sibtus sordide alba undulis fuscis; rachi remigum $\mathrm{r}-1$ albida; rectricibus 8 mediis nigris apice rufis, lateralibus 4.4 albis nigro. u)aculatis.

$f$. major: genis juguloque minus maculatis. Linn. S. N. ed. Gmel. 66r. 36. Nilss. Orn. 184. - Retz. 'Pn. 140. Sc. paludosa. Su. Zool.

Si. Stor Snappa, Dubbel Hechasin.

nab. ad paludes in pratis inundatis non infrequens

-) Scolopax paludosa Gmel. species distincta, mini. me huc referenda.
(D. Lindroth) ubi nidulatur. Vescitur insectis, rermibus et conohylits parvis. Ova $\mathbf{3 . 4}$ oblongo-ovata lutencentegrises. maculis uieris et fuscis praserlim in extremilate

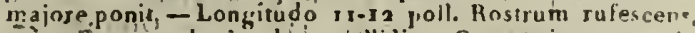
- picr fuscum, basi subtás piallidius. Capur nigram levia - fronla ad ncciput pallide ferruginea maculisune pervis rufis. Lora nigro-ftrsca. Iris fusca. Corpus supro niqroat pollide rufetcente-variegatum, subtus: sordide albun máculis fasciisque undulatis nigrisi Gula slbids. Cauda p:rourit rotundatai rectricibus. I6. Pedes viridercenle-cinernts - 3 i. gallinngb:" (autrunno) vertice nigro: ta:uia ,pallide ferruginta; sublus, alha immarn. lata; reuligis $t \cdot 1$ margine exteriore albo; re. ctricibus r/fo (vere) coloribus splendidioribus (hierni) inigis in cinereun.

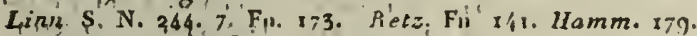
Nitss. Orn 185- Sy Zeo!

Sv. Beck asiuSnä p pa, cukel. Reckasin, ITors".gïk. N. Mikn'gouk, "Trold'ukke, Myrelir:s, Dobbelt Sneppe, Sineppe, Myrsueppe. - Myrebok, Horsegiöy.

Ior. $\beta$ : albida, maculis rufescente-fuscis neputosa. Nilss., l. c.

Hab. ubijue sat frequens in paludosis; miǵrans au. fomito revenit trimo vere. Nidificte huter graminal. carices et ponit ava 4-5. viridescentia maculis sparsis brunneis et aigris. Cilqus ejus ur priecedenlis. Volitat calerrime etalie, criaps: $n \ddot{a} c k$ er $\ddot{b}$ el $k \ddot{z}$ esch $k \ddot{a} t s c h !$ - Longilodo co polt: Rosirum griseum ad apicem fuscilm, subtus pal. fidius.ipmo iapice luberculalo. Iris fusce. Color supra fere ul in pracedente. sud dorso postico nigricanie maculis uenstersis a!bis. Lora uigro.lusca el inacula auricularifuscescente. Gula alb da; zande albidas punclis luscis. Sub. tils alba: jugulo hypenchondritsyue fusco-maculatis, pectore abdginimeyue inimaculolis Cauda rotundaca: rectricibus If b'asl nigris, celdo ferruginescentibus fasciis angustis nigris; lateralibus $1-1$ albis obi alize forruginex. Peclos pallide visidescontes. 

4. gallinula: capite luteo.rufo: tenia lata nigra; abdoınime alloo; cauda nigro-fusca: re. ctricibus 12 margine rufo-variegatis.

Linn. S. N. 244. 8 Retz. Fn. 142. Nilss. Orn. 187. Sy. Zool.

Sv. Hö̀ Sn ä p pa, Hârsnäppa.

Hab. in paludosis el pratis uumidis minus frequenter; viclus, migralio el nidificatio pracedentis, sed ova 4.5 oblonga viridi-albicantis maculis rufescentibus ponil. Sonat tanlum interduns $k i t z$. - Lungitudo $7 \frac{1}{8}$ poll. diostrun hasi carulescens, ad apicen inigrim. Capus supra luleo-rufum nigro-maculatum: lamia a fronle ad occipul nigra, lora nigrn-fusca; cervice fusco-rufa punctis a'bis; gula alba, Lacera colli, jugulum et superius peclus fer. ruginescente-alba fusco-naculata. Pectiss inferius, abdo. mell et femora alba. Dorsum et srapulares nigra virideet purpureo-nilenlia, rufo-fasciala fasciisque luleo-ferrugineis. Remiges nigro-fusci : secundariis apice albıs; inimi colore lorsi. Cauda cuneiformis: rectricibus 12 acu. minatis, nigrn.fiscis, inargille rulu-variegatis. Pedes viridescenle-incarnati.

") digitis exteriore el medio basi nambranula nnitis:

5. grisea: fusca: marginibus pennarum frrugineis; subtus alba: pectore sordide lutescente fusco-maculato; rectricibus 12 albis fusco-fasciatis.

(ascrate) colore superno maris rubro-vergente nigro-luteoque variegato: collo antico pectoreque rufo-brunneis; tectricibus alarum cinereis albo-marginatis.

Linn. S. N. ed. Gmel. C58. 27. - Nilss. Orn. 186. Sc. Paykullii. Sv. Zool.

Sv. Grî́ S n äppa.

Hab. in paludlosis Lapponize rarissima, D. Baro dc Paykull, verisimililer migrans ab America seplentrionali ubi prinum inrenia esl. Hisloria ejus bucusque inco.

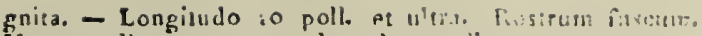

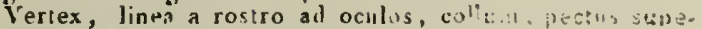
rius el tectricas alsum bruunen-grisea. ! men ill!eralizris, pectus inferius et abdnmen albs. Collism ?usitic, cin :sum, scapulares et remiges intimi fusca maruine pe.narum luzeo-ferrngines. Dorsum pusticnm, uropygum $e_{i}$ crissum alba fasciolis nigricamubus, in lectricibus cqucix undulatis Cauda fasciis alternatis numerosis albis fuscisque. Pedes riridescente-Julei.

\section{G. 66. ACTITES $\left.{ }^{*}\right), S_{\nu}$. Strandsnäppa.}

1..limosa: (hieme) cinerea, ferrugineo inmmixto et rachibus nizricantihus, pectore fisco-Indulato; alis nigris macula alba; cauda nigra, basi alba.

(astaco) jugulo colloque ferrugineis nigropunctatis; dorso et scapularibus nigris pennis ferrugineo-marginatis; dorso postico caulacure nigris', basi caudx nigra; pectore abdumineque albis superm: undulis ferrugineịs nigrisque; basi rostri aurantiaca.

Linn. S. N. 24\{1, 13. Fn. 172. Retz. Fn, 145. S solopaxNilss. Orn. 165. J.imosa melanura. Sע, Zool.

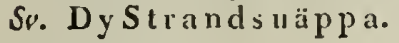

-) Hab. gener. Scolopacis alis medincribus, sed rc. mige t:o longissimo; pedibus longiorabus gracilibus femoribus plerumque supra genu uudis, digito medio exteriore meinbrana ad urburulun primum usque unitis, digito postico ad basin articuluso. Hoc genus dissincsun esse eam a 'Trynga guan Scolopace aucrores nostri avi conseutiunb. 1.1 vero Limosam, us adjeclivum lalinum eljam piscium generi a Salviano attributum nomen, denominarc, inepcum habuinus, quare Aclijes, a greco $(u x \tau(\tau), c$ ditteralis, aucboritate $D$. Illiger praferenda esh. 
jist. sapute supro g,pisen-fusco striis ferru-

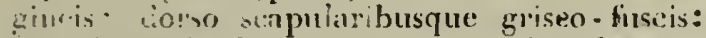

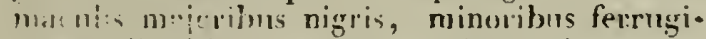

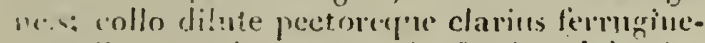
$1-$ striis pectoris trausversis fuscis; abdonsuce corsoyue albis.

llab. in paludosis non infrequens, rarn ad lipas inaritinas; ridificat inter granina, ibi ova 1 obscuse olivacea marulis majoribus pallide fuscis ponens. Insecrie, realuibus et spesunatibus ranarum vescirur. Vexpere soual sty gu p! - Longiludo 25 poltoiel ulirn fiosirum ausanincull apice nigro. Capul cinerenn maculis parris linscis er ferrugineis, culero surva colore cimelesoforrusulle li is brunued. Orbita albida. Lora fusca: Collum औBi: e. jugulum pectus supering et hypochondria grisea;

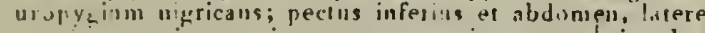

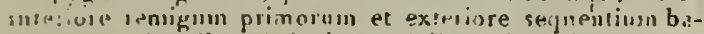
sislite cauda alba, Récirices niedixe arice albie. Pedas nighn-fuscescintes.

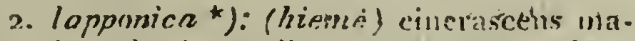
alis nigrofisscis medio vennamin, subtus alha; collda altha fasciis 89 fiscis, ungute digiti medif brevi, edenticulato.

(astreca) m. capite supra rufísente striolis firsci-; linc:" oculari gulatane colose palfidiore, celern subtus claro; dor sen, scapularibus, techicibuque alarum nigris, peunis rufeicentemarginalis, alis cinereis peouis albirlo.matr. ginatis.

*, ..". nomen onecificum a Linnere dobum." quum sicutes jam diL in Lspponic nota fuit, in rufum Brissnili eo minus mutare potuimus; quindo hrec avis ad nullan mutclionem coloris induat "rufum. f. inajor: colore minus claro, sublus lute. scente, dorso scapulariburne obscurc-liscis, ubi mas niger.

Linn. S. N. 246. 15. Fn. 174. Retz. Fn, 146. Scolorax.Nilss. Orn. 364. Limonsa rufa. Sע. Zool.

S2. Lappsk Straids n̈̈ppa, Auzustisnäpla.

jun. obscme-fusca: penuis margiue isabellinis, sed tectionm alarum albis, collo, peince hypochondrisque rufescente-cinereis striolis fuscis; gula abdons inerge albis; monygin tectri-

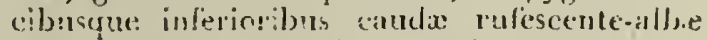
a!iss, mantis lanceolatis nigris, apicibus wchricum albis.

Hab. rulgaris ad ripas maris regionum septentrioualium, ubi nidulatur; emrgrans gregatinı mense dujusıi in terras meridionales, sevenit primo vere Insecta, los eorum, vermes, conchm bivalves felusque piscium cibum e. prabent. Clamore vehemente, claro, sibilante et valde monolono cognilus est - Longiludo 14-15 poll. Rostrum ad basin purpureo-lividun, a! ice nigrum. Iris fusca. Caput supra el collum cinerascenlia striis nigricantibus; supercilia, guld, pecius, abjoinen el crissun alba. Dorsum et scanulares grisescenles: rachibus pemurum nigrus er margimbus nigu...tuscis, dossum inferius, uropyfium of re. crrices candx a'bn maculis mguirantibus variegald. Ale griseo-fusca : rachibus pennaruns tectricum nigris markinibus aibis. Pennx rectricun margine interiore albo-nigroque lascialx, margine exteriore fere unicolores, apicibus altis. Pedes nigri.

\section{Nat. 23. RTCURVIROSTRTIDES, G. G?. NEA *), Sv, Vadare.}

-) Hab. gener fere Carilis, sed digitis plus mirusve membrana unitis. Hoc genus uc proprium ef distinctuen 7 , th is arrisit; nomen vero Nilssonii, Glorlis. 
I. semipnomata: bunnescens: rachibus fisco-macnlatis, macula alarum inferiore alla, sublus alba; cauda fusco-cinereoque-fusciata; digitis semipalmatis.

$f$. paulo major.

(asirale): capite colloque albidis striis $f_{11}$. scis; dorso alisque cinereis maculis transver. sis l. sagillatis nigris maculisque rubris; sub. tus alba maculis juguli peclorisque rotundatis in lateribus faciformibus fuscis; rectricibus 2 intermediis nigro fasciatis.

Linn. S. N. ed. Ginel. 659. 33. Scolopax. - Nilss. Orn. 165. Clotris - Sv. Zool.

Sv. Halfhinnfotad Vadare.

jun. capite supra tusco, obscuriore fusco. variegato; nucha cinerea immaculata; peu. nis dorsalibus scapularibusque fuscis marginibus rubresceutibus; rectricibus fuscis, basi 2 mectiorum alba, lateralibus extus fasciatis; subtus alba lateribus colli cinereo-striatis.

Hab. in Uplandia, D. Paykull reste $D$. Nilsson, sine dubio errans ab America sepientrionali. qux patria ejus est propita of vulgaris, $D$. Willson. Cunchyliis bivalvibus, vermibus insectisque marilimis viclitrt. Nidifical inter graunina in locis paludosis ova 4 sat imagna in extremilate una aruraque in altera obscure olivacea maculis nigrufuscis ponens. - Longitudo $\times 4-15$ poll. Rostrum plumbeo-cinereum. Iris niguicaus Supra brunnescens: raclubis. fusco-maculatis. Collum anticum et peclus cinerascentia striis parvis fuscis. Gula, pecius iufurius el ab-

quia apud gracos alio avis meer cocurnices ita alenominalur, in aliud a $\Lambda_{\varepsilon(1)}$, vado, deriva. sum, nobis potius muscondum.
Somen alba. Alze obecure cinereofusix ad marginem pallidiores usque id colorem albu-cinerasceniem: remig:bus nigris, macula alba. Uropygium lectricesilise couda: alba. Recirices 2 intermedia lusca, cetera cinerascen tes fasciis angustis fuscis. Pedes et menbrana eorum plumbeo-cinerei.

2. gloctis: obscure fusca: marginibus pennarum luten-albidis; subtus alba: alis inferne strialis; cauda alba fusco-fasciata; pedibus lutescente virirlibus.

(astrate) capite collnque super nigro a!boque striatis; dorso seapularibısyue nirris marginibus pennurum illius albis, haruu rubre. sceute albo-maculatis, toctricibus alarum majoribus rubrescentc-cincreis rachibns nigris; subtus alba: facie, collo autico pectoreque maculis ovalibus filscis.

Linn.'S. N 245. 10. Fn, 17r. Retz. Fu. 177. Hamm. Fn. 83a. Sco!npax. - Nilss Orn. 166 . Glollis cbloropus. Sv. Zool.

Sv. 'Tung Vadarc. - N. Höstfugl.

Jun. pedibus cincreis.

Hab ad lluvios et lacus, rarn in ripis marilimis $\mathbf{S c a n}$. dinavize boteslis xestate, et ibi nidifical Pisciculis, conchylits parvis ovisque pisciun vescilur; agiliter cobum in 2 qua ad ventrem usijue vadans collo extenso caplat. Yellio cit tgia $t g i u$ ! el sibilat evolans tudut, el sedens $\varepsilon u k$, larritus criat $k r a ̈ h$ ! - Longitudo $82 \frac{1}{y}-15 !$ poll. Rosirum cinereo-fuscunı. Regio inter llandibulam superiorem el oculos, sub corpore toro dursuque medio s.ba. Capite el cullum supra cinereo-fusca maiciubas perisarum alb.dis; dustum supernum scapuldrev el tectaciss alaruan nigro-fusia, marginibus penuaruli futeo-albidis. Liemiges primores 11:fru.fusci, secundali: cipelen-fusci larero interiore albo-vas tgal. Caudx alba: recurribus 2 laterslibas sp-rse fusco-sritistis, sed intermediis fuscu-lascialis. Pedec lulescente-viricles. 


\section{G. 63. RECURVIROSTRA *), Sv. Upnäbb.}

1. (zlricrpilla **): pileo cervice alisque nigris; nacula inedio alarum magua alba. Linn. S. N. 256 1. Fn 19r. Retż. Fn. 169. Nilss. Orn. 152. R. avocella. Sv. Zool

Sv. S v a 1 t höfda d U pu äb b, Skärfläcka, Alfit.

Hab. Iantun in litoribus mariliunis Gotulandize et $S_{c a-}$ nire. In locis lilormu inundatis nidum elevatum pyramidale dificat, ubi ova 2 oblouga alha griseo-maculata ponit; valde limida criat vulitans tvil tvit! - Caro sopidissiunus. - Longundo 17-18 poll. Rostrum nigrutn. Iris rubro-fusca. Pileus, cervice collumque postice el superne cireutamque atarum nagnum nigra, cetera avis alba. Pedes cinereo-crerulei.

\section{Nal. 24. TANTAL EIDES,} G. 69. FGATHEUS '*), Su. Helgspof.

1. falcinellus: castaneus: facie atro-viridi,

-) Hab. gener. capite parviusculo, collo longo, rorpore ovalo, alis acuminatis remige primo longis. simo; pedibusque eractlitus longis: digitis 3 anticis semipalmatis ad articulum secundum usque, digibnque postico brevissimo. Nomen lalinum.

-) Quando Gesnerus rerum nzsuralium nomim ab aliis linguis, quain grae" of latina fingere solebas, ea semper alficere nobis lex fuit. Quare nvocellam, a lingua gallica foctam, in atricipillam, quo have species a congeneribus jactle distunguicur, mutavionns.

-..) Hab. gener. Nenmenii ; sed remife lin rentigilus 2 er 3 longirsumis breviore. Nisseistientibus auctoribus, quanam esset Egrptinersm lbis; hoc no. men uvis in historia insignis jis : us ut specilitum coluservari credidimus, acieuque figatheum a graco

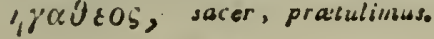

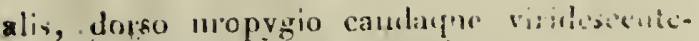
aturs a'nco-violaceornc-ustentibus.

f. ininor.

Lim, S. N, 241. 2. Rer3. Fo. 175. Hamm. Fo. 175 vilss. Orn, 160. \$v. Zool.

Sv: Rie Helgs pof. - N. Rylle, Drmsmcryx: junior: pennis capitis colligue sumproris ni-

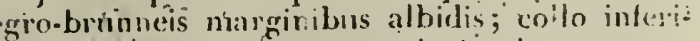
ore abdónimeque cinereo-nigrin: theso sempl:laribusgue fusco-cincsẹis alis cuudayue min::s viride niterit,bus

Liın. S. N. ed. Smael, 64, R. Tantalus viridis.

idlus jun. colouc obsculione nigricaute cinereo el marginibus pentrarum cilpitis collique lutiore albis.

Hab.: in cumisalu Norregize Jarlsberg et Gorlindida,

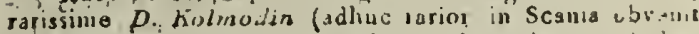
nec, nisi, romimugraus) D. Nilsson, fuxia lacus preiuciesgue. Insecta, rerises, cunshylue et regetabilia cium ei prabeut. - lungiludo m. circa 2 pej. Kusirum rivideacentemgiam ad apicem luscum. Iris, brualęa. Bricula ocplanis iuda virde-aisens. foscia grisea circumdaia. Co.

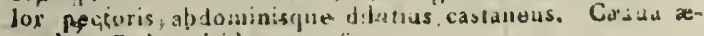
qualis. pedes viridescente-lusci.

"G. 70. NEOMENIUS *), Sv. Manspof.

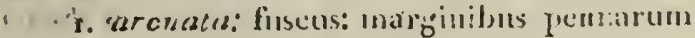

-) Hab felier corpus abovobun : collo elongato: a lis 'medoucrabus, reinige $1: 0$ lungissarial; postibus

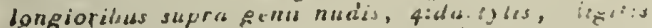

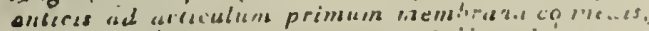
digito postitu terratn langente. Woinen hue its-

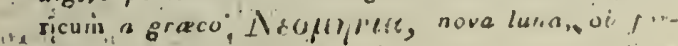
rinm rostre derivatum vićetur; Gun autem siro. : moisur scribecsur. 
cinerascentibus 1. rufescente-albis; subtıs albicans: striis fuscis; canda cinerascente-alba, lusco fasciata.

$f$. colore pennarum inarginum magis cinereo, in durso et scapulálibus rufescente-albido sordidiore.

Liun. S. N. 248 3. Fn. 168 Rers. Fn. 136. Hamm. Fn, 177. Scolopax. - Nilss. Urn. 16r. Numenius. - Sv. Zool.

Sv. Bâg nä b bs Månspof, Storspof, Tullare, Lảngnäbba, Kourpa, Vindspole. - $N$. Spuve, Langneb, Teelspove, Regnspaar, Reguspove, Spue. - L. Gurgastak. jon. rostro loreviore, parum arcuato.

Hab. ad ripas fuvi-rum et locuum sal frequens; nidulans in pratis paludosis sed locis elevaris, ova 4-5 olivacea maculis undulis!fue fuscisel nigris ponens. Emigrat autuman et tevertit primo vere. Victital insectis prasertim Gryllıs, vermibus, hirudinibus, concliyliis parvis et graminbus. Sonu ejus duplici ho $i$, ho $i$, $i$ ! vel $k$ lay $i b-$ tri, tri! bene cognoscitur. - Longitudo avis 16.20 poll. et ultra, rostri usque ad 6 poll secundum xtatem. Kostrum fuscum apice nigrum. Iris brunnea. Caput el collum cinerascentia maculis rachialibus fuscis. Subuns albicons: striis rachialıbus sparsis, fuscis. Mlargines pennarum in dorsin et scapularibus rufescente-a!bi. Dorsum posticum album maculis sogittatis nigris. Podes obscure cinerei.

2. plaxnpus: obscure fuscus: marginibus pen:ıarum dilutioribus; stria verticis lutescente alba: alis allo-maculatis; cauda fusco-griseia, fasciis obicurius fusco-fascidlis.

Lithn. S. N. 243. 4. Fn. 169 Retz. Fn 137. Hamm. Fa. 178. Scolopax. - Nilss. Ora. 162. Nunseuius placeopus. Sv. Zool.
Sv. Grifotad MI ả ispof, Smípof, Qrid. bondc: - N. Surale Spue, Giasspoui, Gaasespon.

Juntor: roslro breviore, vix $1:$ poll. longo, partum arcualo.

Hab. intra circulum arcticun sat vulgaris, in provin. ciis meridionalıbus minus frepuens. Insectis, veinubus collcliylissyne parvis vescilır. tmigrans autumo advenil primo vare. Nidificat un pratis liunudis, ubi ova 4 ponts. Nite volitans clamat hy hyliy hy dlat-Longitudo 16 poll. Rustrum cieruleo-migrum, basi rubiescens. Iris brunuea. Caput supia lorayue fusca, siria verticiv lulescente-alba; lateribus capitis, collo pecloreyue grisescente-albis stuis interdum sagillatis in hypochondriis sublascifounibus fuscis. Gula aiba. Dorsum, scapulares et tectricas alarum obscure-fusca inaculis marginum pennaruni albidis. Dor sum ponlicum, abdomen et crissum alba. Pedes plumbei: unguibus nig̣tis.

\section{G. 31. PELIDNA *), Sv. Gråsnäppa.}

1. Subarquaca: cincreo-fusca: rachidons subuigris 1. nigro-maculatis: fironte, superciliis, gula creporeque sublus allois; rachibus margnubusque rectricum interioribus allis.

(aescrbe): lionte. supercilis gulatgue ilbis,

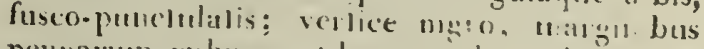
penuarutu rubrescenlibus; nucha riblurisconle nigro-stlatla; subtus tere caslat ca fusco-1ludell.

- Hab. genu. inter Tryngas et Neonienios cadit, quare nunc ad hos nune ad lllus hac aris nuinelofa est. lllustr. Cisvirl ist proproum gellus slobult, unde incer Janialpides posuemus, ques a Jiyngis peabus differt altioribus et jemoribus congiurabus. Helidis - lleicdelas, juscus, derivata. 
Lata et alho-rariegata; teciricibus caudx alhis nigro. et rubrescente-fusciatis; dorso, alisque superne nigris: marginilus peislarum ferrenge nescente-marginatis; cutula uggro-cincrea, alboinarginata.

Linn. S. N. ed. Gmal. 658. 25. Scolopex - Rezz. Fn. 163. Tringa 1slandica. Brïnn. Orn. 55. 180. Tringa ferruginea. Nilss. Orn. 179. Trings. -- Sw. Zool.

Sv. snarböjd Grasiai pa.

jun. similis var. $\alpha$. hieme; sed dorsn, sca. pnlaribus tectricibus pue alarum nigro-cincrcis, futescente-albo-marginatis; remigibus ad apicem intus albo marginatis; pectore immacu. lato lutescente-albo- et fuscescente-tincto; pe. dibus fiuscis.

IJab. in lilloribus Scandinavize borealis, ubi nidificat, ora $1-5$ lutescenlia maculis fuscis ponit et 26 dies inclibet. In provinciis ausiralioribus primo rero vel autumno sereno sub $\mathrm{m}$ gralione obrenit. Edit insecta, vermes et varias species Fuci. - Longiludo $7 \frac{\frac{1}{6}-8}{4}$ poll. Fiosirum nirum; lines injer rosiruin et oculos lusca. Iris tirsca. Penne nuclales albicantes medio fusco-striala. Collum et pectus etjam albicantia, fusco-striata, sed culore magis claro. Rectrices $\mathbf{x}-5$ cinerex margine interiore et basi albx, intermedia 2 longiores acuminalx, cinereo-fusc marginibus tenuibus et raclibus albis. Pedes fusci vel nigricante-cinerei.

Nac. 25. RALLIDES,

G. 72. RALLUS *), $S_{v}$. Knarr.

-) Hab. gen. Corpus ovatiusculum; collo brevi, sile mediocribus rolundatis: remige 1:0 breviore quoin 2, ; et 4, qui longissimi sunt; pedibus sat longis, validis, parte femorts brevi nuda; 4.dactylis; digitis 3 anticis plane fissis. N. gea. latinum, jom jun Aldrovendi.
I. aquaticus: supra nigricans: margini. bus peunaıum lufescentibus; subfus caculco. griseus: hypochondriis niguis albo-fasciatis.

Lınn. S. N 262 2. Fn. 195. Ręz. Fn. 176. Hamin. Fr. 208. Nilss. Orn 188, - Sv. Zool.

Si. Vatten $\mathrm{K}$ nar. - $N$. Strandrixe, Slrand. snarre, Vandsnarre, Joldhone, Vindiöne.

Jun. medio pectoris inferioris rufescente, abdomine nigricante sunicolore.

llab. ad nquas, apud nos raru:, in Norvegia vero sxpius obvenil; migratosius. Insecla, lunibrici, cuuchylia et regelabiha aqualica victus ejus sunt. In tumulo jug. dam inter arundiues vel juncos nidificat ora b-1o lutescentia maculis rubro-fuscis pollens. Strepet $k r r i p$. krrip! - Longitudo $9 \frac{\frac{1}{8}}{8}$ poll. Kostrnm rubrum, mandi. bulze superoris apice et suprema parte fuscis. Iris aurantiaca. Gula abida. Latera capilis, collum enticum, pectus et abdomen creruleo-grisea: undulis obsulelis rufescentibus. Hy pochondria et asillares nigra: striis trons. varsis albis. Canda brevis, cuneiformis: reciricibus nigris olivaceo-marginatis, tecricibus inferioribus albis. Pedes sordide incernati.

G. 72. ORTYGOMETRA *), Sv, Qveuknarr.

-) culmine rosıri absqque plaga froniali nuda. t) digitis absque margine exserto.

r. crex: nigricans: marginibus pennarun cinereis rufisulue; alis rufis; abdonjine albo.

-) Hab. gener. Ralli sed corpore compressiore; ai.'s *.e

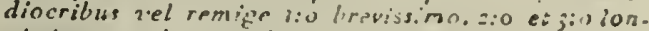
gissimis, vel remigibus 1.3 cequali':us $4: 0$ longis. simo brevioriuus; pediuus longis: digitis fissis, medio longitudine bibia vel lungiore. Nomen hoc genericum Ariscocelis a graco OQT matrix coturnicum a Brissonio adoptature el a ten antiquies, guam Gallinula Latbani. 


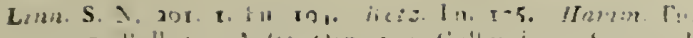

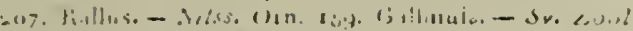

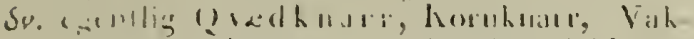

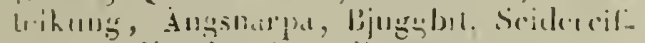
ner. - N. Ahervixe, Shotrarate.

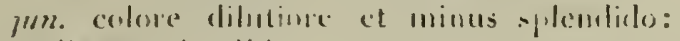
tuaculis salaris allis.

llals. uhinge sal the juens inter segeles a in mills

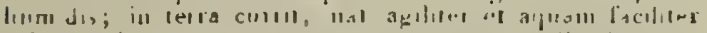

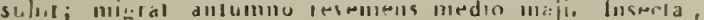

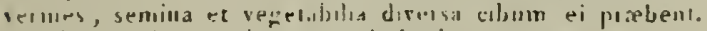

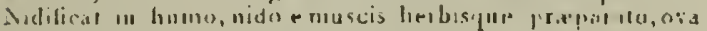

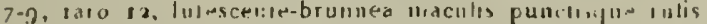
ponens. Vesplete continue whil: hrey. hreg, irrp,

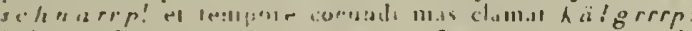

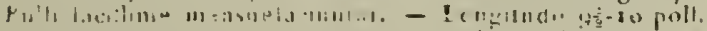

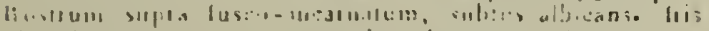

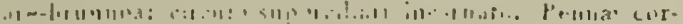

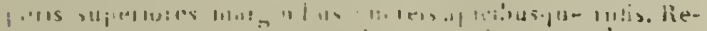

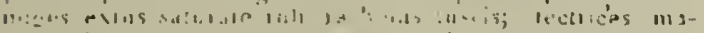

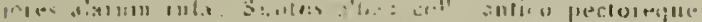

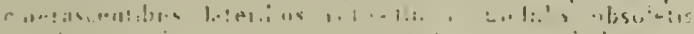

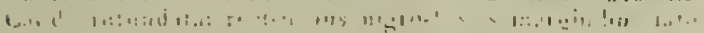

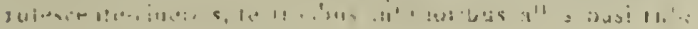

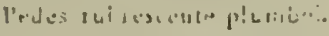

\section{it}

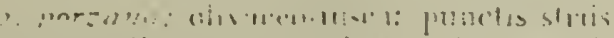

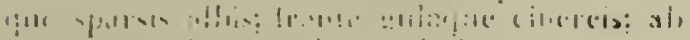

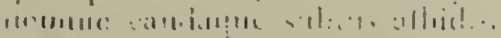

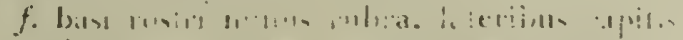

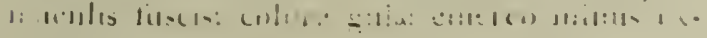
1. 31.

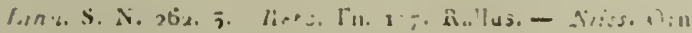

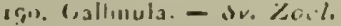

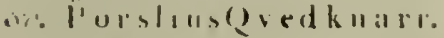

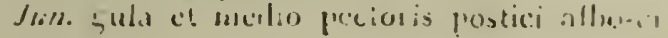

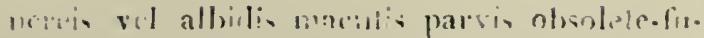

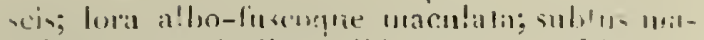

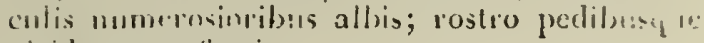
ririrlescentu-! iscis.

Hal. intri arumlines et equisela ad aquas dul.a, $\ldots$.

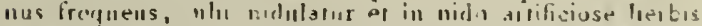

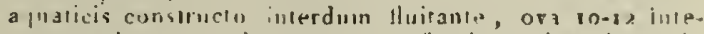

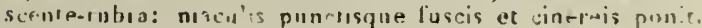

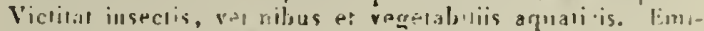

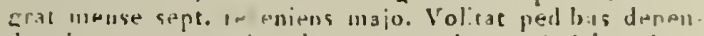

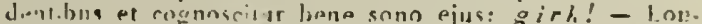

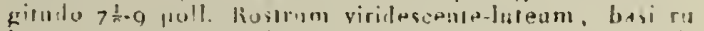
bruni, (autumno: "ivisceo vilide, spica linscum.) Iros la-

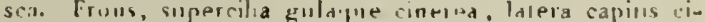

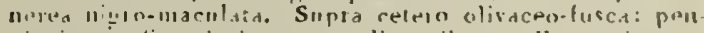
nis in inedin nigris et maculis striistille ally,s variegalis.

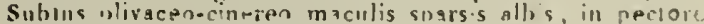
rolundaris, in Hvpochnadris fasciolieformilus. Cabth runeiformıs: rectricibus nigris olivare.s-nurgillatıs, medlis 2 marginibus tenubus albis. Pedes vinidescente-ulute.

-) culinine ed basin dilaras piagam nudam frontalem formante.

3. chloropus: griseo-nigra : alis olivacen. fincis temus allon-maninatis; fronte muta cher. mesina; sublus ciemleo-tincta; caudar strhtu. alloa rectriciluts modiis nigris.

$f$. colore clariore.

Linn S, N. 258. 4. Retz. Fn, 873. Iulica. - Nilss. O:1. 191. Gallinula. - Sv Zoo:

So. Gulurinlots (? redkuar.

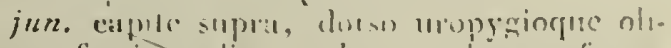

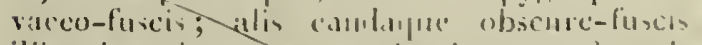
illis clare bumber-marginatis; macula suleoculari, gunla colloque antico albidis; cetro.

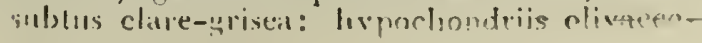


tinctis; rostro apice olivacro-viridi, hasi nifvaceo-fusco: plaga aj,icure olivacea; iris bruuneal ; pedibus sub-u!ivaces:

Linn. S. N. ed. Gmel. Ü37. I. Fulica fusca.

Adlus junior: culure clarrore circomque rostrum albida; plaga fromtali rubra vel lutescentc.

Linn. S, N ed. Gmel. 7or. 17, IS, Ig. Fulica maculats, Aavipes, et lisıulans.

Hab. imprimis in sylvaticis ad ripas aquarum duhum, oquisata sat raro inter arundines; mexpit, natal et whirat celersine, caudam moret perpetuo, y̧uirscit iuted in fruticibus; ex inseclis, vernibusque, seminibus el herbis aqualicis victilat; nidulatur in arundintetis, ova 5.8 civerascente-a!ba nlaculis parvis rubrescemtibus bis per all. num poners diesque 21 incubans, facile mansuescilur; clamat keck, reck, forr, kilh, kick, vel gi,gi? et girgikylh! - Longitudo 2 $^{-1 / 4}$ poll Iris, plaga frolleslis et rosirum chermosint, apice rostri lintec. Subtus com lore magis creruleo tuncto: abdoinine medio albo-uebuloso e: liypothnodriis albo-striutis. Cauda supra mera. Podes ":lessente-virides, apicibus femurum chermesiais.")

Nat. 26. ARDET:TDES,

Div. $x$. Digitis parum palmaris :

(i. 7f. IXOBRYCHLS **), Sv. Rördrom.

\% Futica Porphyrio Rel. rel porphyrio hyasiuthinots Teirin. indigenius Liuropa austrulis, verisim.

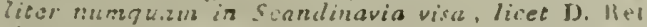
ins sentuerit, "rble hanc avem o fauno neot"r at+clissimis.

Hit. gaver. fene Ardere; sed collo plus minusve -assion fentoribusque plemanque parum nubis. gen. e guncess vocis J SOS arundo, et pinukw

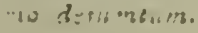

r. Ininntus: capile lavi stupra, durso, scitprilardnus, remigilus secundaris candaque nir gris viride-nitentibus. Femoribus totis plul!nosis.

Linn. S. N. 240. 26. Nilss. Orn. 158. Ardea. - Sv. Zuo? Sv. Liten Rïrdrom.

jour. striis fuscescentibus, in pullis patucioribus, marsinibus pennarum superiorum rubrescentibus; renigibusque caudaque nigris.

Linn. S. N. ed. Gmel. $6 \bar{j} 7$ 58. Aidea daisubriatis.

magis jun. capite supra rostropuc fuscis; collo antico albicante, lateribus capitis, nucha, pectore, dorso tectricibusque alarum rublescentc-fuscis striis firscescentibus; remigibus rectricibusque obscurc-fuscis; jedilsus viri-. dibus.

Hab. in Vestntannia rarissimus, D. Nilsson. Victiras pisciculis et ranis, pracipue vero insectis. Nidifirat in arundinetis, et ova $5 \cdot 6$ alba ponit. Timnilus clamal: $g \ddot{a} c k_{\text {, }}$ rundimelis, et ova $\tilde{a}_{c k}$, $\ddot{a} c k$ ! - Longitudo $15 \frac{1}{\varepsilon}$ poll. Rosirum luteum, apice luscum. Iris et regrio ocularis lutea. Latera capitis, colfum el tectrices alarum rufo-teslacea. Subtus cetero aibus, testaceo tillito.

2. stellaris: capitc lavi; corpore supra ferruginco undulis fasciolisque nigrts; subtus pallidior maculis magnis longitudinalıbus nigris; pennis colli tmuniclis.

Lirn. S. N, 230. 2\$ Rétz. F11. 132. Act. Soc. pbyciongr. Lund. I. p. כos. Nilss. U11. \$59. Asdea. - Sy. Zuol. Su. Si jern-tröld Rin drout.

Hab. in locis paludosis arundinetis, passina; ubi nidulatur et ova $3-5$ viridescentia ponil. Rane, pisces. insecla acpuatica, vermes et conchylia cibus ejus suna Volitansalteclaind, lete ut Corvus: hro a h? in reiraver.

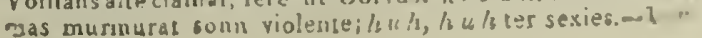


giludo a ped. 5-4 pol!. Maxilla rostri superior fiusca u: gine lutescente, inferior, regio acularis pories jue rilidescenle-lutei. Iris Iutea. Kanizes niglicalltes lestaceo-lasçiatı: tectricibus nigro.undulatis. Cauda testaceu-fusco. que variegata.

\section{G. 75. GRUS "), Sv. Trana.}

r. cinerea: cinerea: rortice nudo, papilInso, rubro; fronte, nuclia, collo antico, remigibusque nigris; pennis scapularibus lungissimis; ab oculis teura collari alba.

Linn. S N. 234. 4. Fn I6n. Hęz. Fn. 129. Hamm. Fn. 174. Ardea Grus, Nilss. Orn 15ti. - Sv. Zool. 14.

Sv. Grá 'I'ra il a. $-N$. 'L'ranc.

Jun, vertice plumoso; colne nigro occipitis collique antici mullo 1. maculi-formi, unde trenia alba collatri nulla.

Hab. passim in locis paludosis provinciarum uraseriim boreslium, nbi nidificat in liuticelis pripcipue alnetis; ora 2 magna vuidescente ciures maculis fuscis pnuens et hebdoniades 4 incubans. Serpetes seminaque herbarum palustrimin victus ejus sunt. Emigrar altumno et revertit rere, volilans +xcel,e.el clamans: 1 , gorr!-Longirudo 3 ped. 8-10 po!l. kinstrum viulescente-atruin apice pailidius corneo et hası. Iubuescente. Iris brunnea. Frnns $\mathbb{S}$ lora pilı niguis densis, verlice raris. Tectrices'ultima cineren nigrx. Inngiores laxie supra apirem alorum ulrinqua dependentes: radiis fibrosis. Cauda rollindara apice nigricanle. Pedes nigri.

) Ilab. fener prizredensis, sed collo pedibusque longioribus, magna porte supra genu nuda, digibo postico altius in libia sito; nlis mediocribus: remige 1:o serunde breviore, qua fere longifudina ercii longissini; secundariis ad corpus proximis orruabis \%. brevissimus subulabis. N. genger. labinum.
Div. 2. digitis sutpalnratis:

\section{G. 76. CICONiA *), Sv. Stork.}

1. nlha: alba: orlsilis nculorum mulis, rem:gilus scapularibusque nigris; rostro pedilussque sanguineis.

lirun S. N. 235, 7. Fu. 36r. Retz. Fn. 130. Ardea Ciconia. Nilss Orn. 154. - Sv. Zuol.

Si. II vit St nik.

jun. colore alarum nigron fusco-tincto rostroque lubro-nigro.

Habl. in Gothia, rarn in Golltandia vel supra Scauian, ubi nidifical in teclis, turribus 1 . arboribus alis decollatis, ova j-5 ochracen-alba pnnens. Mligitns autumno mense Aprili revenit. Talpis, inuribus, inusielis, aviculis, ovis avium, serpentibus, ranis (non vero bufonibus?') lacertis, pisciculis. inseclis prosersim gryllis victital. Mures viros pullis adfert, ut ad illies raplandos adsuescaniur. - Longitudo 3 ped. $5-6$ poll. Iris brunnea.

2. nigra: nigricans: prectore, ahdomine, candacue subtus albis; rostro, orbitis oculnrum muda gulaque chermecinis: pedibus rubris. Linn. S N. 235. 8. Fn, 163. fietz. Fn. 131. Ardea. Nilss. Orn. 155. - Sv. Zonl.

Sv. Svalt Sinik, Odersiala.

Jun. rostro, orbitis, cula pedibusque nlivacen-viridilus; capite colloque rubro-brumueis: margiubus pennarum albicantibus, corpore

") Hab. gener. Ardere approximat; sed! guln nuda exsensili, alis mediocribus: remigibus $3-5$ longissimis, $2: 0$ breviore ef $1: 0$ adhur breviore: pedibus longis, 4-dactylis: digitis 3 anticis inembrane ad orficulationem usque lim connexir.postico cum anficis horizontali, ungusbiusque brevibus, deprcssis, absque denticulis, Nom. gener. larinum. 



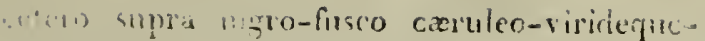
(1:3:10.

Ilich. Fasin in paludosis arberelis eljam Gotulandix. Vidulalur in arbnribus rewomun arcticarum el ora 2.5 sordide viridescente-alba punchs interdum sparsis fuscis 1. mit. Vescitur ranis, pisciculis, gryllis insectisque diresis aliis. - Lungiludo 5 ped. Color colporis niger in -iolnceum et viridem vergens. Itis brunnea.

\section{G. $\left.77 . \operatorname{ARDEA}^{*}\right), S_{v}$. Häger.}

1. candid $\left.{ }^{\star \star}\right)$ : fola alha: capite cristalo; (1),110 viriclesiente-luteo; apice sxpe nigro; regione oculari virudesente; pediJus longissimis fuscricentilum.

Cinn. S. N. ed Gmel 6rg. 34. Ardea Egrelta. Sv. 2.00l. S. J, lanklivil llager.

imm. capile levi; penuis dorsakibus longis mi!lis.

mizgis fun, masdibula rnstri apice et in cul-

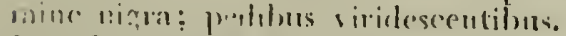

L:37. S N. 237.0 24 Fn. 166 . Retz. FII 154. A. alba. llable sine dubio lonlaila visa, in Scania ad Araslöf, 7). T.zind, in Vre!mannia D. Thusuluege, rarissina avis. Molalin in orburius, ova 40 palluderernlea ponens. Vercilur ran., lacellis, piscivulis, iuvecis velnubusque aqualicis-lonizilledo ped. 10 poll. Csista capitis depen-

- Hab. gell. Alw mediorres renige l:o breviore quam siv et 3.o, y.u langlssmat; pedes tongi graciles: Jemuralius plus minusue supra genu nusis, 4-cbectyI1: disiso infelib et arlico cain éclimo inembrana brevi connexis, postow cum antirs horizoncalio Wunı. gen. listinum vecur:um est.

- Numen spertiorum Ginmini e gallico derivatum hatad noliss placuic, quare Brissuni nomen retinuimass. libst wor rarissime. Srandenaviam forcevisise. verit, e lisura non sbjicienda.

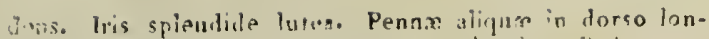

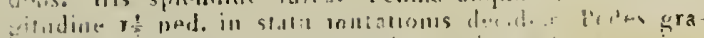

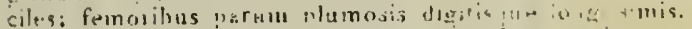

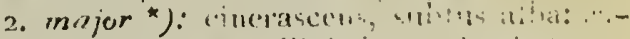

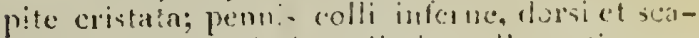

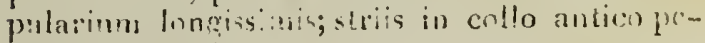
vinecrue nisilis.

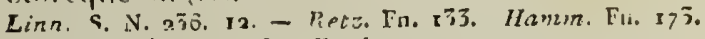
Ardea cingrea. Sr. Zool.

Sv. Stor ll ä r r. - N. Fiske Heyes, Shidbeyre, Skriclizeyre.

jun. caprite lavi 1. subcitstatn, fante vertiaeque ciacrois, mantihula rostri super niernfuscu: Inaculis et mandilunla iuferinre lutess; pennis longissimis nullis, collo pallirte-cinereo

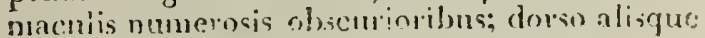
cipludeo-cinercis albicante rel firserscente variesatis; pedibus migro-cinercis: patrle femormm nitda linlesice:'tc.

Hab. a Scunia Snacie ad Vilrociam usque Norregua

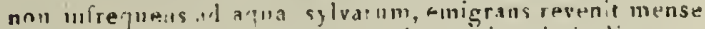
Marion. Vicilat inuritus, avicali: ranis, pisciculis, con-

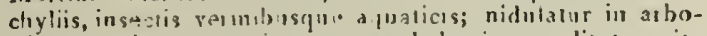
ribus excelsis et ponit ova $z$ - 4 thalassing, volitat rapire retraclo; clamat kraik! at pelicit $g a n k$, $g a a k !-l, n 11-$

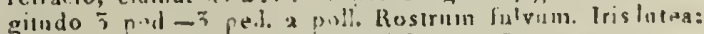
reguione noulaıi $n$ ida cerulen-violacrs. Frous el allterior

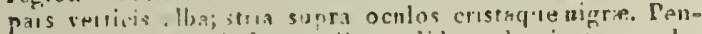
"re lougssumn sidbulate collesalldidee. dousi et scapuleriam argentax. Coltum postice cineriascens. Dorsum et trurices alaum carbala-cinesea, pedes fusci: parte te. inorum nuda lubiescerre.

- Noner specificum Lahami, cineres, cume fruc cinerea furile commusecur. "ude denoninationen Linnel huic $\mathrm{A}_{v i}$ in celace atulsiore dabum, pre. ferre magis nutavimus. 

'rhl, 3. LEUROHHAMPHA!.

N'at. 2.\%. PLATYRHAMPIIDES,

c. -S. PLATYRHAMPhUS $\left.{ }^{*}\right), S_{v}$. Pla:ınäbb.

1. pusillus: niger: manginibusque proman tum rubrescentibus; finciio 2. super witus mblececonte-allis; stria a rostre ad menlos firsea, rostro rubrescente-cincreo apree nizoro; rectricibus mediis nigris, rufo-maryinatis.

(astate) capite supra nigrofurso, inperciliis allis fusco-punctatis; stria rostrali funconigra: lateribus capitiq alluidis, nucha cinceren, hypodondrisque albis fusco-strialis; rostro rigin, hasi rubrescente.

Eechst. Naluig. Deulschl. Vul. \& p. 352. Numenius. -

Hah. in Coutlandia rarissimus, a me capus el cum cnllectiune Aviim Svecarum fese cumplrla Hef Acad.e mix Sciensiarna donatıs; sine dabio prjant in Nonvrgua boreali. In!la circulam arcricum nidilical al invecta vermeque édit - Longitudo $6 \frac{2}{4}-6 \frac{1}{9}$ poll. Riosirum rubirescens, apice nigrum. Caudo cuneilormis: recllicibus 2 medis lungissinis, laleralibus fusco-cinteis. Sublus al. bus. Tionie, nuclia, lareribis culli, precole, hynochinndris, lecuiribusque caudx inleriosibus "lgro-striolatis. Pedes viride-rinerei.

-) Hab gen fere Tryngx, sed claracterihus genericis bene distinctis, unde ustoribus inodo ad Trinhas modo ad Numenins relatus e'st; os rostrum ejus depressum a nature heic locum ei assignatum esse videtur. Niom gener. a gracis vocis Tiket? depressus el pieflyOS rostrum, derivalum est.

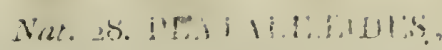

G. *) 7?. PLATALEA **, Sv, spadribb.

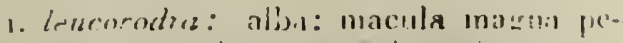

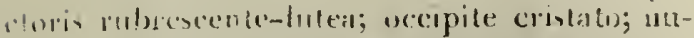

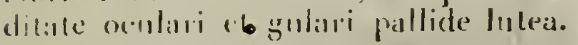

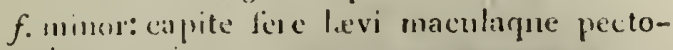
ris vix colispicilat.

Linn S. 太. 23 1. 1. Fn. 160. Retz. Fn. 128. Humm. Fu. 172. Nilss Urin. $15^{3}-S_{2}$. Zöol.

Su. I! vil Spalluablo: praze: Prlikan.

jur. t:ajile l.ev!, ruslon olsscure-cinereo;

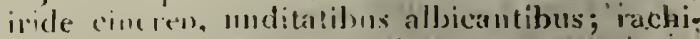
bus apicifn-que remigum primorum nigris; - mesecular pecturis mollas.

$H_{62} l$ in cstiis Dariorum Norr- at Vestrobothaiz; ut a Vorwgix borestis, tarissima tanien; einigrat el revenit cum Cyunss. P'iscicutis, ovis pisclum ransurumgue, unseclis!? vialubus, conchyliisque viclitat. Nidifical in arboribus, III fowlobus et raro uller arundines, or dzalba, fiermillue maculis rubrescenlibus sparsis, ponens. "Langlludo pred 6 poll. Rnstrum longiludine $8 \frac{1}{8}$ poll. nigrum: canstrulis exrulescentubus apiceque ochraceo.

-) Rationem, quam haluerit D. Nilsson Pusynoihynchum fygnituall inter aves Scandencizice enumerass excogitare, haul putavionus, quando nec svecia llec Norvegice visa est hatc avis.

", liub. gener. fere Auseris: alis mediocribus, rm. plis: remage l:o fere longitudine $2: \ldots t$, qui longis. simus; pedibus longis, iofututus, 4-doctyles: digltis 3 enticis ad articulationem 2:m membliane late emargensta unicis, pestico lougo antecis hortzun:

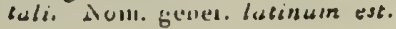



Ins rubra. Crista occipinis pemnis fore 5 poll. longi, a. cumenati, depeodentibus, Alx longitudino canile. I'e. des nigri.

\section{Ord. 3. PTEROPODAE, Nat. 29. FULICIEIDES, G. 80. LOBIPES *), Sv. Flikfot.}

I. hyperborsus: (astate) nigricals: marginibus pennarum ferrugineis; gula pectere abdomineque albis; jugulo lateribustgute colli rufis.

$f$. regione oculorum rufo cinereoque immixta; jugulo lateribusque colli minus rufis, cinereo immixtis; maculis hypochondriarum, et supra, majoribus et numerosioribus.

Linn. S. N. 249. 9. Rerz. Fu. 152. Tringo. - m. Nilss. Orn. rg3. Phalaropus cinereus. Sv. Lool.

Sv. Nord pols Flihfot.

junior: capite, dorso, scapularibus rectricibusque 2 mediis nigro-furcis, peunis rulomarginatis; alis nigricantibus: ınarginibus apicibusque albicantibur; firnte, et suhtus albil; lateribus colli lutescente- et pectoris lis pochondriisque cineren-tunctis: tilnits intus linteis, extus digitisque hutesicente-viridis.

Linn $S$ v. Nd. Gmol 6:5 33. Tringalusca. Eruinn. Oru. 37. Tringa lubata. Nilss. Uiu. I. c. iar. A. e: $B$ ?

- Hab gener. fere Phalarnpodis, sed praier characie. res equerivos digitis ab articulatione primn laceralicer lobatis, caudo longa rosundaba. N. gener. bastrum.
Hab. ad oquas dulces regionum Scalldinnvien intra c.r-

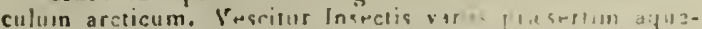

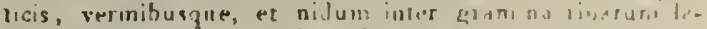

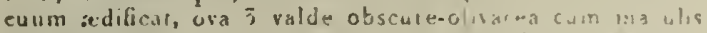
numerosissimis nigers nolirus. - Jonkrifurto 7 nos. I:0.

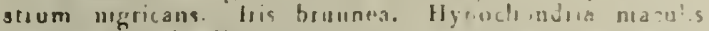
maznis cineleis alba. Dorsum, scapulares, lecllicas a eruat. et recirices a median nigha: peinnis suberiolis dorsi ot scspularium late ferrugineo- et tectricum alarum an al ce

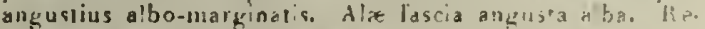
ctrices latelales cinerascentes anguste cibo-margh tiala!. Fe des viridescenle.ciuerei.

\section{G. Si. PHALAROPUS *), Sv. Barfo:.}

I. fulicaria: (hieme) supra capite cinc:io macula ad aures in farcia nuclialiabroutro nie gricante; dorso, lateribus pectori-, scirmulare. bus mopygioguc carulescente-cincreis: s!lbtus allues.

Linn. S. N. 240. 8. Fn. 179. Tringa lobars. Retz. Fn.1:a

Tringa lisperlores f. Hamion Fil 187. Si. Zoril.

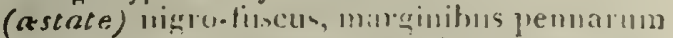

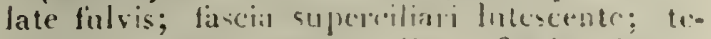
ctricibus alarum apice allots, fascia alartun alba; nopygio albo niģro-fasciciato; subtus lilfus.

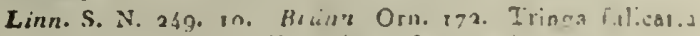

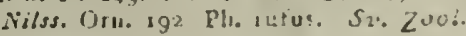

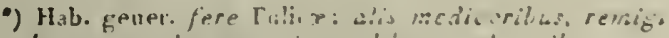

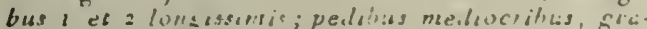

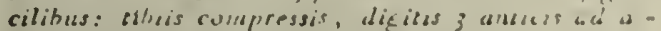
ciculabionem t:m unztie, citieru laberaluser dellsicbo-

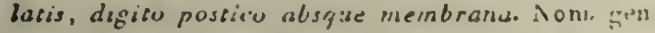

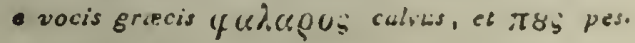


$-10$

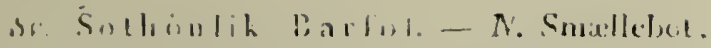

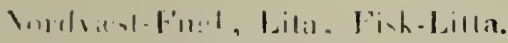

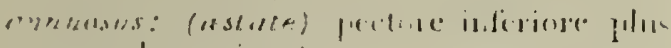

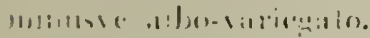

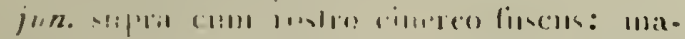

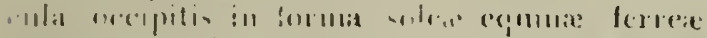

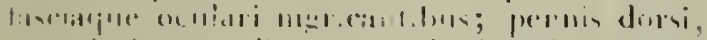

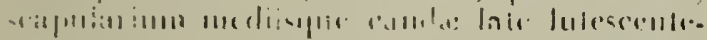

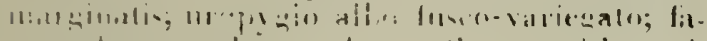

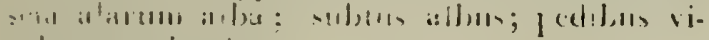

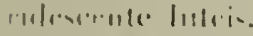

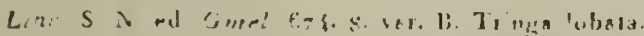

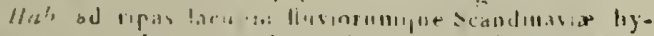

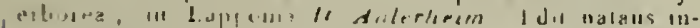

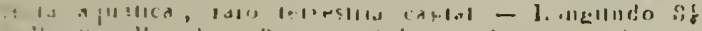

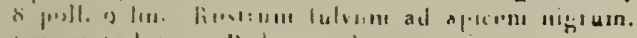

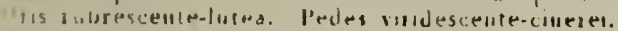

\section{G. S2. FULICA *), Sv. Sorhina.}

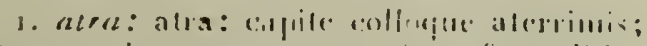

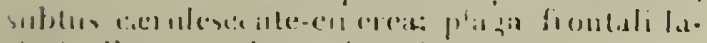

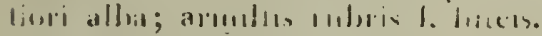

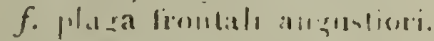

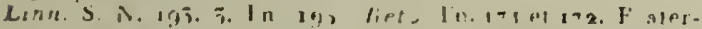

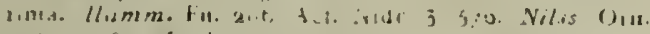
1115 - 80 Lowl

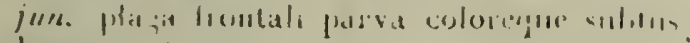

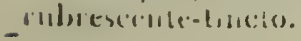

1 adilic

" Nabil. genter. fere Colle; alis mediore lue: remige

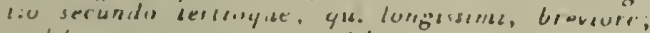

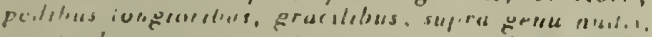

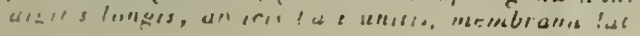

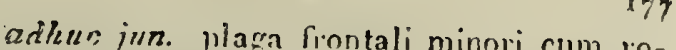
plapa fiontali minori cum ro5ton perlilsusque olinaceo-cincrea; sublus cinerascens.

Sparm. Mas is F. mt!ions.

Var, \%. allia: alls allsilic. Sparrm. Mus. 12, 15. leucorix.

Hah in poitudosis, ad laces at ginus marg ron in fiequens. (rar. ? ad Ifolmam, D spusmara mun in avis composita, paste Culeb. D. Tomminek, Ponlis et

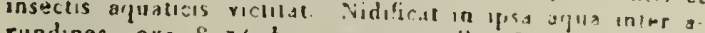
rundines, ors 8-14 brunnescenlis-nibe fus-o- al lubrescente-punclata ponalls. Clamirat: gäh, gah! mt nias interjum etjan dod, dod!-Longltudo is-17 pull. lio. sirum abum, roseo-tincluns. Iris chermesina. Peries ci. nerei, videliacti: ormulla rubra l. !ules.

Nat. "̈o. HODICEPITIDI:S. G. 63. PODiCEPS '), Sथ. Gumpfor.

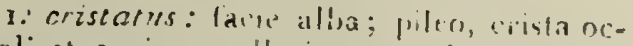

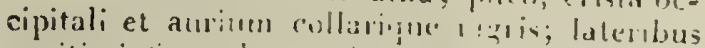
capitis infororibus rulio; corport sublus, colln

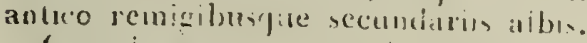

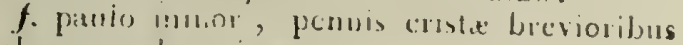

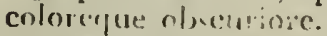

Labit. S. N. 72\%. I'n. 151. Colynibus. - Lieiz. Fu. 110. Nilss. Oru :95. - So. Louk.

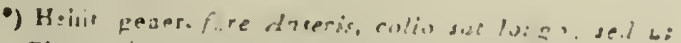

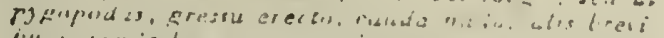

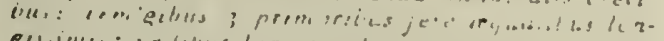

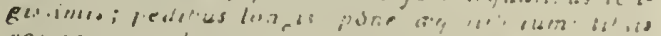

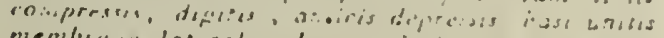
membiais laserale cobules, diegrio posins c017.

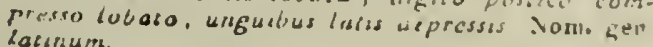




\section{$57^{8}$}

Sv. Tof s rumprot.

biennis: crista brevissima, albo marginata; fascia iregulari nigricaute a rostro sub octrlis ail ucciput.

jlinior: crista nulla, fronte facierpe albis, fasciis flexuosis ut et in collo superiore; iride lutea; rostro rubrescente-livido.

antuc jun. capite colloupue superiore fuscis. Linr. S. N. ed. Ginel. 505. 3. F.ulvmbus urinainr.

Hab. in ripis ef liteonibus sai fie flens, ingralorius.

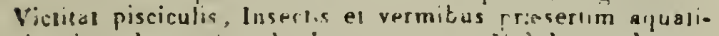
cis, interdum eljam lieibis oqualicis. Nidulatur piertinque in aqua cum nićn gransusibus ałualie.s constru=10 sepe Aluerite et ora 3-4 bido-virida, fusco-undulara. Clan:at Gurrer! et pellicil gurk! - Longiludo 10 - ao poll. Rusirun capie iongius serdicie iubrestens, mose moxtem ruhidas, supre luscum apice albo. Fris rubrothermesins. Nutilas a rovio ad oculos rubrs. Color sibins argentes-abbus. I alesa pectoris abdominisanc et iuguium ruia. Pedes exius nigricarles, intus luteo-albi.

2. rubricollis: pillo cristaque occiputali brevi, truncala, nigris; gala laleriblarme capitis inferioribus ciremen: collon levi misn.

Linn. S. N. ed. Gmel. 5?2. 2/4 Colymbus -570. 18. Colymbus subsisislus. Keiz. Ia. I15. Nilss. Orn. sgtiSu. Zool.

So. Rödhals Gumpfot.

jun. supura nigin-lissels: capite licvi nigro, lateribus inferioribus allsis, nigno striatis; in-

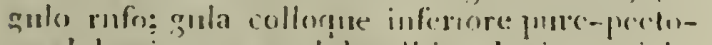
re abdominergne sordide-iblbis; lisis rustri $l_{1-}$ ude-lutea, iride rubrescente-luseal.

spa.rm. Mns. 9. Colymbus Parolis.

Hal. ul prisr minus frequens cum aodem cibo as nidilicalions, ora 3-4 albido visidia, luteo-fuscuque-sor- dein Pallicit keak keak! I7!

d'dis. Pallicit kenk kerk! - Lnngituda ri. if! roll.

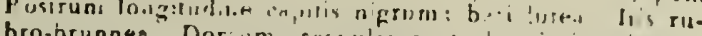

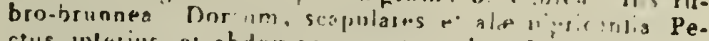

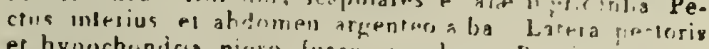
et hypochendirin nigm-fuscu inarulala. Rmoiges secun. darii albi. Pedes exlus nigli, insus liateo virides.

3. auritus: pileo, crisla ocripilali brevi.

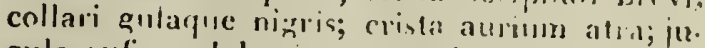
gulo rufin; abdomine rem'giburque secunde. yiis albis.

junior: rrista amsium millu: albedine inte: fostrum nculusege listine in collus descendente: mandibulis panlo recortalis.

Lonn S. N. 202. 8. Fi:. $152114 \mathrm{~mm}$. Fis 150. Pudb Aree ed. De Geer. 212. Culynibus. - Fiels. Fn. 111. Files Orn 197 Su. Znol.

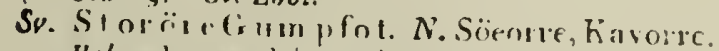
Hab ad aquas dulces vuigaris, rabuls ad hiensa ras

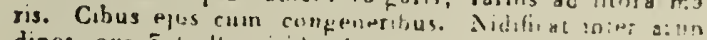
dines, ova 5-4 albn viridis, brinueo soldidia peoness be

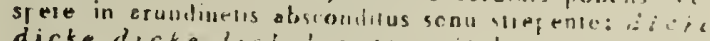

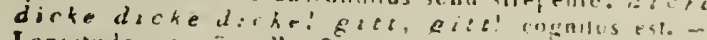

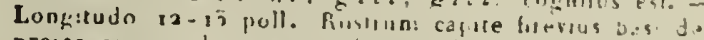

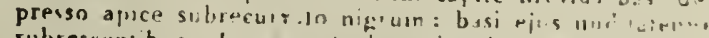

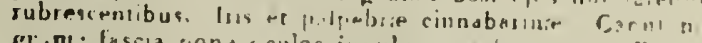

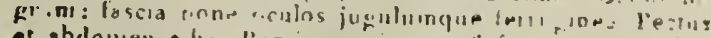

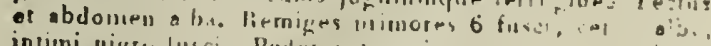

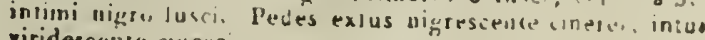
viridescrumaruedei.

4. obsemrlis: pilen, co istarpure auvium ma?.

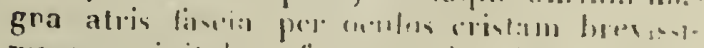

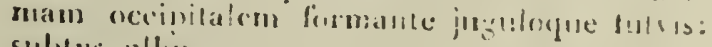
subtus allius. Linn. S. N. ed. Comel. 59r.16. Colgubus connuins $\therefore$.
Znol.

Sv. mörk Gumpfot. 

jur. nigro-fureus: copitu: lavi; genis, gria, ab)lamine

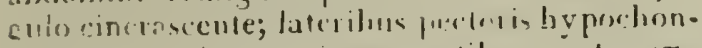

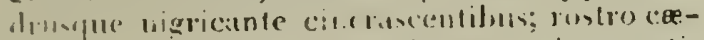

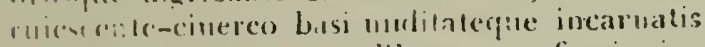

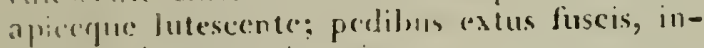

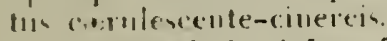

Linn. S. i. ed, Gmel. 5q2. 2j. Colymbus. - Ress. Fn. iti. Nilss. Orn. 108

Hab. ad aquas dulces, rarius in lilloribus maris non -iraquens, sed cum pracedente, rul sumilis, commusalus. Sidum in arcindinetis ex berbis cedilicar fluisantemet ora

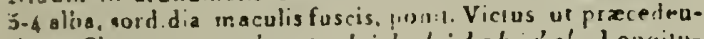
riun. Ciamat sonu vibrante bidebidebide!-LongillSo $12-15$ poll. Riostrim nigrum: basi et nuditate inter rosirum ot ocuins roseis npiceque rubro. Iris lutes: circulo

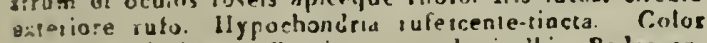
cuperaus nigricas. Remiges securdari olbi. Pedes exas nigrı, inlus grigei.

†. minor: olivacers-uigricans: capite lavi upro grulaque atris; latovibus anticeque colli

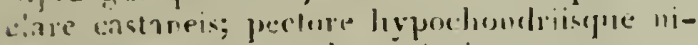

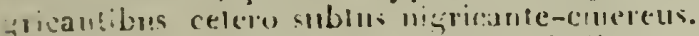
Exan S. N. ad. Ginel. 591. a8. Luigmbus hebridicus. Sv. Zno:

S\%. li il bu in p fot.

iuriol: supua cl latcribus colli albis fascio-

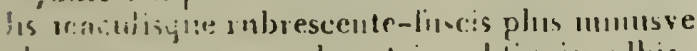

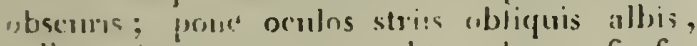
colio antiso, pectore et sululus ciare rufis. fe:-

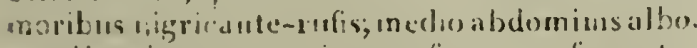

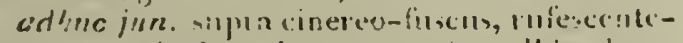

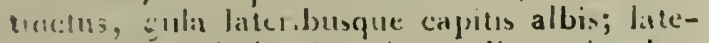
ilin: inlli pallite cinereis; collo antico hy-

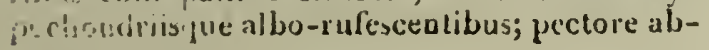

domineque aibis; rostrum fuscum: mandibuia inferiore el margine superioris luteo-cinereis; iricie brunnea.

Linn. S. N. ed. Gmel. 5ox. 20. Colymbus, - Retz. Fn. 184. Niles. Orn 199.

IIab. Ad agnas dulc, rarior, inseclis oqualicis rictisans: nidum flutanten inter arundinas conotrult el ara 45 viridescear. alba 1. rilesceal.h. Lrunner-sordidis. po-

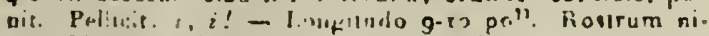
grum; basi in idibule inf-rinris et llud 2 ce intor instrum et osulos. eque illso alth is. lils r ifescentebrunnea.

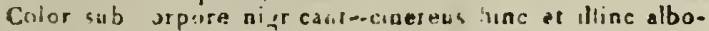
imsnia'lls. in femnibus t rrisso rubreacente-linclus. Romignes ci-ereo-fusei: basi et pluma pennarum secundarialin: ircistori albis. Pedos extus riride-fusci intus iasasaali. 



\section{Subclass. 3. HYDRORNITHES,}

\section{Ord. .. PYGODAE,}

Nat. 3o. COLYMBIIDES,

Dive x. pedibus 4-dectylis.

G. 84. COLYMBUS *), Sv. Lom.

1. glacialis: capite collogue viride-atris, collari jugnli antice et colli postice latiore al. his nigro-striatis; corpore supra nigro maculis: quadrangularibus albis; subtus albus.

Linn. S. N. 221. 5. IIamm. Fn. 158. Nilss Orn. 206.Gunner in act. Nidros. 3. 125. Brünn. Orn. 154. C. torqualus. Sv. Zool.

Sv. Is Lom, Inmer: N. Vashymber, Havhymber, Brusen.

jun. capite colloque cinereo-fuscis: punctis cinereis albisque in geurs, colloque anticoalbo; corpore supra obsrure-fusco marginibus pennarum cinereis, subtus albn; mandibula superiore grisescente, inferiore albicante; pedibus extus obscure-fuscis.

Linn. S. N. 222. 6. Erünn. Orn. 129. C. Imner. Gunn. in act. Nidros. 3. 240.1 . 2. E. 1.

-) Hab gener. Podicipitis, sed alis Lrevibus: remige $1: 0$ longissimo. Cauda enguse a brevissima rucundate. Hedibus pene aquilibrium mediocribus, tibis compressis, digitis onticis longissimis mem. brana incegra unitis, digito postico basi articula. 10. membranula laxa. unguibus planis. Nomed gea. e verbo eraco ynilirifrer meron. 
Hab. are arcticam in insulis, ubi nidificat in ripis aquarun dulciun ova 2 isobellina maculie plus minusra mapnis purpureu-cinereis pouens; raro litsora ausiraliora visilal, acmen harengurum, cibum ejus parucularm, pernequens, quanvis ova pisciun, insucla el vegersbiliamne rina non dedignatur. - Longiludo $27-29$ pall. et ulira. Rosirum nigrum: apice cinereo. Iuis fusia. Culen rapilis collique d viriden el caruleum accedil Prina dursiles ot scapulares maculis ad aficenı 2 quadrangularıbus, sed teciricum alarian punctis pluribus nilris. l'edes exius ni. gro fusci, intus cun membrana albidi.

2. arceicus: capite collonul postico fusco. cinereis, antico violareo-migro: fascia angusta gulari, regionibus ad aus:s taniaque collari albis migun-strialis.

Linn S. N. 221.4 . Fn. 150. Rerz Fn. 106. Ilemm. Fn. 157. Gunn. in acs. Nidros. 1. 216 6. 3. 6. 1, 2. Nilss. Orn. 207.

Sv. Niordpols Lom. - N. Hyuber, Imlere, Ilaviulere, firmer.

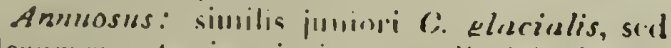
plerunque truia nigricante collari in lateri. bus colli.

Biennis: colore cinerco capitis nucheque ohscuriore ad fionlem nigrolu-lincto; collo in. tico violisuro-nigro albo-variegato; corpore sup:a nigro lationlis maculis albis; nondibula superiure nigricante, bisi vero inferioreque cincreis.

Thionnis: similis var. $\alpha$, sed pennis albis spar. sis in collo anlico.

Hab. in regione circuli erclici, ubi nidificat intergra. min. I erundinas riparum aquarum dulcium ora fusca maculis spasis nigris ponens el migral utumno ad refiones meridionales el sat frequenier obvenil. Vicritol raabbus, piocibus, iesectrs planfisquue aguaticis. - Longitudo
26. 28 poll. Rnetrum nigricans. Iris fusca. Colnt capilis cinerells ad liunient el latera saisim in uegrum linclus. Dor-

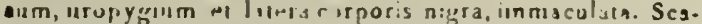
pulares nugra mar:ulis puadrangulatibus abis Tectrices, alaum maculis monoribus ipasis altus. Subtus albus, sed collo inforiorm nifio-striglo. Pedes exlus fusci, intus cum membrana slbid.

3. septentrinumlis: gula listeribusque ca.

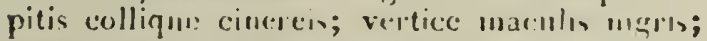
jugulo rufo; coceipite collurjur postico inferio. reque rigricantilums albo-striall s.

Linn. S. N. 22n. 3. Retz. Fn, 107. Hamm. Fn. 156 Gunn. in ace. Nidros. 1. 244. 1. 2. f. 2. Wilss. Urn. 208. So. Zool.

Sv. nordlig Lom. - N. Loin. L. Gahhor, Gakalte.

Jun. similis val. $u$, sed collo antico tolo albo pennis rufis sparsis, nuculisyue supernis minuribns \& sepe lntersentilus.

Linn. S. N. ed. Gmel. 586. 16. C. sirialus. Brunn. Ora. Iis C. bureslis.

-Adhuc jun. supra capile colloque nigrocinereis: pennis leviter albo-mangiuatis: corpore nigro-firce: inaculis mmerosis minutis albis in marginibus pcunarum sparsis; tertrir.um alarmm apicibus albis; subtus albidıs: rostro albido-cineren supra obscuriuge ; Iride biumea; pedibus extur fuscis intus ailidocinerejs.

Linn. S. Y. ed. Ginel. 587. 87. C. stvilstus.

Pullus: nigin-luseus sullus allidus.

Fab. in regronibus hyparboreiv vulgais, cusumno ad meridionales emigrans. Piscibus, ovie encum, rsnis, ineecris herbisque equalicis viculal. Nullfient ul confepleres ora 2 oralia oblongiasim. olivaceo-brunnes maculis 11 . merosis fuscis poosas. Clasnital: kooih, koaih!-Lo i, 
gitudo $27-24$ poll. Rostrum nigrum. Iris aurantiaco-brun. nea. Corruss supra nigro-foscinn, in adultissimis imme. culasum in allnis $5: n-4: 0$ inaculis minutissimis slbidis vix distinclis, sublus album. Pedes exlus virıdo-nigri, istus el memibrana digilum livide-albi.

Dirs 2. pedibue 3.dect jlis.

G. 85. URIA *), Sv. Stjertlom.

x. Brissoni $*$ ) (hieme) cinereo-nigra se. ricea; sublus alba; alis'unicaluribus: remigibus secundariis tantum apice albis; pedibus obscur'is.

f. inare parlo minor.

Linn $S$ N ed. Gmel 585. 14. Hamm. Fn. 154. Recz Fn. 809. Colymbus. Nilss. Orn.2u5. U Troile. Brünn Orn. 110 U. Srarbag. el IIt.Ringrie. - Sv. Zool.

Sv. Brissons $S$ t jer tlom, Silladoppilly, Sill. knipa. - N. Leügive, Lcnivie, Lumbe, Laugvire.

-) Hob. gener. fere generir antecedentis, sed prater clsaracteres genericos tartis gracilsbus, digito postico plane nullo. Nom. gev. e voce grace $8 \rho \eta$, cauda.

- Nomen specificum hoc in memoriam auctoris'hujus generis pro Troile l Troille l. Suarbag, Ringvia. Ec. uf inepea pretulimus. Uriam Brunuichil Sabin. locum in Fauna Scandinavie mereri, quia mare glatiale sontum 'fiequertot, th hine verissmiliter litsora Norvepia visitat, licet a nutlis aucsoribus ibidem observata, vel polius cum Uria Brisnoni, a qua samen pedilus viridesoensibus, gula juguloque antico. brunnescente-nigris soriceis et longisudine is poll differs, commucase, opinio nosira manes.

ascace: cnpite, regione oculorum gula collo. que postico firseis sericeis; nre intus clare linteo. Linn $s$ N. 220. 2 Fn. 140. Culynibus Troile. Brünn Orn. 108. U. Lomvia. 109 U. Troille.

jurb. sirnilis aduixe hieme sed rostro bréviore cineren basi littescrite; colore superno cinereo-fuscon tinclo; linea pone oculos vix distiucta, transenute vero ad maculas ciues eas; collo antico etjann cineren immixto; sublus sordide-alba; pedíbus livide-luteis membranis fuscis.

Hab. xshale in maribus hypesboreis sat frequens. ubi nidulalur in cavilatibus rupiom el ornm sat m.gnum oblnngum acurum viridescens l. cxerulescen, maculis slriis. que irregularibus nigris punit. Hieme ad maria asiralioris emigral. Piscibus, imprimis clupeis, vicrisal- Longiludo 15.16 poll Rnstrilm cinereo.nigrum : ore inlus fivide-liseo tris brunnea. Supra nigre in cinereum vercens, subius a'ba : lipea pone orulos io reginne alba nigrs. Pedes lutescentr-fusci: tibiis postice membranisque nigris.

2. nigra ) hiema: atra: macula magna alarun alba; genis et subtus allis; pedibus rubris. $f$, minor, nigra: macula alarum ininore. Zinn. S. N. 220. 1. Fn. 148. Hamm. Fn. 153. Colyınbus Grylle. Retz. Pa. 108. Nilss. Ora. 204. U. Grylle. Sv. Zool.

Sv. svart Stjertlom, Iste, Grisla, Gresla, Grylle. - N. Teiste, Teste. L. Zhialhe's; pull. Boryek.

(astato): tota nigra: macula alarum majori; ore intus pedibusque lere sanguiueis.

-) Nomen Grylle, quo inrola Goblandire hanc avem siguifleanc in quod Bussonius dederit, merito mu. cevimus. 
Jun. nigricans: capite, colln inferinge latcribusque pectoris griseo-allucque-ntarublatic, pennis variis albn-cinereis inter s(ap)u!ares et in uroplygio; macula olarmu cinerco- 1. nigricante-maculata; gula pectoreque albis; irisle nigro-fusca; ore intus perdibuserue rubro-livilis.

Hab. ad oras.maris balıbici el necidentalie rulparis. Nidificat ut entecedens et ponit a cra oblollna allide: maculis plus minusso magnis nigris el cooereis prosinlim in Iermino majori. Pisciculi ei crustaces mipores cibum -i prabent. - Longilude $22-15$ poli. Rustrum gigrum: ore iotus pedibusque clere.rubris. Iris fusca.

\section{G. 86. CEPHUS *), Sv. Tånglom.}

I. nanus ${ }^{*}$ ) (hiemo): aler; sublus remigibusque secundariis apice albis.

(astato): capite, genis, gula juguloque atris, cetero ut in hiense.

Linn. S. N. 2115 Fn. 142 . Rebe Fn. 100 . Hamm. Fin 139. Alca Allo. Nilss-Orn. 205 Uria, Alle Su. Zuol. Sv. Pyssling 'L'iluglom, (siöulands dutiva. N. Söekonge, Jacob, Baitrefixrt, Boerfixr, Borfizr, Lille Pcderdrikher, Alle, Lille Grönlandskdue, Hys Thomas, Bys Thumas.

-) Hab gener. incer Urias ef Alcas: proximus camen illi. Aum gener quum minime ud Alcam per. binet el cam forma routr quain modn vivendi ab Uriis deffert, inelico est not.um genus, cuinomen Pale lasii - greco K$K(q)$, fucus, sumpeum dedimur; quia nomen Raji Mergulus, a I) Virllot prolatum, farile in errorem: quasi ad nationem mergijdum pertineret hac avis, duceres.

*) Hoc nomen opecificum a Celeb D. Temminck do. iua pro norvegico allo edemevimus.
Iun. similis adulli hitme; sed rostro adhuc brevinge et genis magis cinereis.

Hab. od nitu glnciale passirn, raro hieme ad orae australiores ma:ios. Nidifical in cavilatibus a fissurio rupium presuplorum, ovum cxeruleu-riride plesumque immacylajum ponens. Viculat cruslacers aliisiue inseclis rermibusque marinis, quze sene inler fucum rolligit. Clamal: roptes, tes. B:ce. tes, ses! - Longiludo 8!-9 poll. Kostrum ingrum. I:Is nigrn-fuses. Ioctrices alas rum majores strus $;-4$ albis. reilur eupernus corporia iuterdum striis nigricantubus variegualus: tune fascia ab-

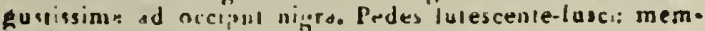
branis riridescente liscis.

Nat. 3r. ALC ELDES, G. 87. MORMON *), Sv. Larfnäbb.

T. arclcus: sly ira cum collari juguli aler, lucens: gula lateribusque capritis cincren-albis, remigibu; nigro-lusecs; sublus albiss; cauda brevi æequali.

Linn. S. N gri i. Fn. 1̧1. Tiesz. Fn qq. Hamm. Fa. 38. Nilss Orn. 202. Alce - Sv. Znol.

Su. Aurdpuls Larliabl. - S. Sriepapegoij), Lunde, Lundfogel, Lunne.

Jun. instin multo nimori, latcribus laribus, Intescente-finsco; reginuc iuter ilfum el veulos nigricante-cinerca; gula loleribusque capitis magis cinerein; juzulo nigro antice sen-

-) llab genver. Alcx approximat. sed choracberibus genericis relecus valde dieferminalus. Ala breved

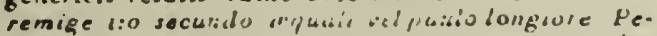

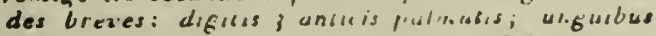

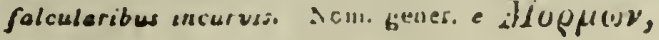
larva. 
sim in nigricante-cinereum; pedibus opacuss rubris.

Brinn. Orn. Inh. Alce deleala.

Hub. ad mare glaciale Veniens initio mensis Maju severtit Aug. Nideficut vat in spelinnculis ierrestribus ro! iu Gisiuris rupium arum albidum, cinereo-maculatum ponens. Vescitur pisciculis, insecus plantisyue marinis. - Longlsudo $12 \frac{1}{7}-14$ poll. Rostrum luipscens, ban cxrulescerite. cinereım. ed a nicem sanquineum: mandibu'a superıor. 3 - et inferior a-sulcata; sinu oris inacula neda lutea. Iris albida. Macula nuda crularis iriangu'nris rubra. Fascia superciliaris cinteroa. Peiles aurantiaco-rubii.

\section{G. 85. ALCA*), Sv. Tord.}

I. balthica *) (hieme): alı, corpore subtus, linea nullia a rostro ad ormlo, vel una furco-interrupta fisciaque alarum albis; allis Inngitudine uropverii; cauda cumeiformi.

Brünn. Orn. tol. Hontoppidon Vurges nal. hist.-2. 107. Alca. Toin. I. t. 36 Su. Zrool

(astate): linea ad oculis nivea hand inter. rupta, genis, gula juguloqile superule: atris berunneo-tinclis; rostro intus clare lutro.

Linn S. N. 2to I. Fn 13g. Retz En, 96. Hamm. Fn. 153. Nilss. Orn 200 . Sv Zoul 44.

Sv. Östersjö I'ord, 'londmule, Tureinule, M ile. - N. Alke, Klıbalke.

-) Hab gen. fece Colymbi, sed collo hrevi et remife 1:0 longissumo longicudius serunili $l$ parum hillgiore, pedetius hievilus: dipiti, cancum 3 ansicis pulmatis ungustiusque parum curvatis. N. gemer. probabiliter ab $\alpha \lambda \%$, , forticudo, quond rostrum, derivasum.

-) Nomen specificum gottlandicum Tordo, ut ine. peum, in nomen Bruanicbul mutovinus.
Junior: similis arlulli hierne, wed gestro me

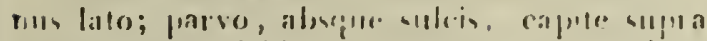

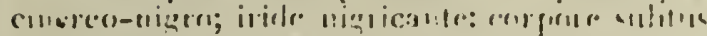

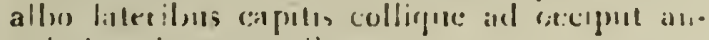
gublim rinetaiceniluts.

Linn. S. X. 210 2. Retz. Fn. 97. A. Pics. Erünn. Orn. 102. A, unisulcata.

Hah. in variis locis Norregire gregatim et sat frequens et imprimis in insulis Srecia: Gulland, Nämdun. Bonden. Orum maguum, oblongum, aibum I. Luieccena miculis vigris l. Puscis irregularibus, sepe punclis eljam cinereis. in cavilatibus rupuen inaccessibilium ponit. quod dies is incubat. Viclitat pisciculis, inseclis crusiacellque marinis. - Longitudo 14!-86 poil. Rustrum nigrum: inlus livide-luseun sulcis $\mathbf{7 - 4}$, quorum medius albus. Iris brunne⿻. Latera canitis cerrascentia lines descendente nigra pone oculum. Pedes nigro-cusere.

3. impennis: alis volatui ineptis, remigibus brevissimis; supua atra, subtus macula. que ovatit ulrinque aule oculos alba; cauda brevi.

Linn. S. N. 210. 5 Fn. 140. Rets. Fn. 98. Hamm Fn. 137. Nilss. Oili. 201. Sv. Znoul.

Sv. vinglïs l'old, lingvill. - N. Peogrin, Anglemager, Geirfingl, Garfirgl, Grufingl.

Hal ad mase glaciale; obronit epe pregatim ad littor Nurregix proneltion in Sündmües; s-d iarissime in insulis Balunsize. Viduliral in fissinls rupueu orunı megni.

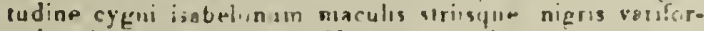

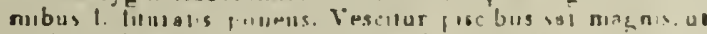
cyclopletis ef flaulls llasiuis ralis. Clinial: a an fla.

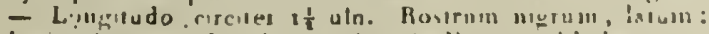
bosi sulro 1. Ad ajiren culces b nlls mond bule superubris, sulcis 8-10 unadibu!re uferiors. Is s nigra. Alx nigric: pennis loco renigum secundariorum apice albis. Pedes nigri. 



\section{Trib. I. LIORHAMPHAE,}

Not. 32. PROCELLARIEIDES,

G. 6g. HALOHIPPUS *), Sv. Hafshäst.

1. glaciales: albus: dnr'so, scapularibus, tectricibus alarum, remigibusque secundariis cæruleo-cinereis; remigibus primoribus griseofirscis; canda conica.

Linn. S N. 215. 3. Fn. ז44. Retz. Pn. so2. Hamm. Fn. 144. Gunn. in act. Nidros. 1. 182. Nilss. Orn. 226. Prncellaria. - S2. Znol.

Sv. Is Ha lislıäst. - N. Havhest, Gröulandsk

Stornfugl.

Junior: clare fuscesccnte-griseus: pennis dorsi et alarum apice obscurioribus; remigibus primoribus rectricibusque griseo-fuscis ; macula ante oculos angulala nigra; rostro pe. dibusuge latescente-cinereis.

Hab. in mari glaciali, in insulis hyperboreis Norvegiz aidificar, abi in cavilalibus rupium ovum magnum album pome. Edit calnem celcrum moltuorum fuilanrium, molluscas el ronchas varias ad cet'ss fixas, pisces, \&c. - Longindo i6 poll. Jiestrnm fluvum, circa narea arantiaco-linclam. Iris et pedes Julei.

\section{G. 90.}

-) Hab. gener. fere Columbr, sed alis cauda longio. ribua: reinige 1:0 longissimo: pedibus mediocrio bus: cibius compressis, dificis ancicis longis palmatsis; loco diesti postice ungue longo aculissimo; cauda rotundate l. conica itulut hujus generis

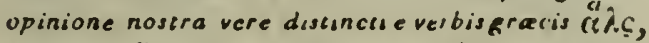
mare, ot ítros, equus; nonien ejus eriviale Norvegia.
G. go. ZaLOCHELIDON" "), Sr. Seomstala.

1. pélagica: nigra: fascia uropygii lata picibusque remigun sc:cundariorum albis; subtus fuliginosa; alis cauda paruun longioribus.

Linn. S. N. 232. I. Fn. 313. Heiz. Fn. sor. Hamm. Fn. 143. Nilss. Orn. 223 Procellaris. - Sr. Zool.

Sv. Hafs St or m s va l a, Stormvädersfigel. N. Sörron Pider, Vestenvinds Are, Sön. denvindsfingl.

Junior: colore paulo pallidiore: margine pennarum fuliginoso I. rubrescente.

Hab. in Oceano boreali raro ad oras Svecie; nidificat interdum in tissuris rupium I. spelunculis terrestribus Norverixe, ovum subratundum album ponens. Victsoe piscubus. insoctis rermbusque in mari fluisanlibas ol cutem cecorum adfixis. - Longitudo 5t-6t poll. Rostrum et pedes ungri. Iris brunnea" ").

-

Nal. 33. LARIIDES, G. gr. CHELIDO *"*), Sr. Tärna.

-) Hlab. Geuer antecerlentis, sed perlibus longisinis. gracilibus caudaque quadrungularas l Juriaba. N.

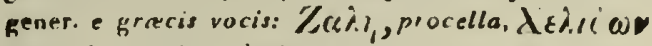
Hirundo inarina, derivalum est.

- ) D. Hammer incer uves Nurzegicas Procellariaun Pufinam (l'socell. cineresin nubis! rensab. quam veru cantion in mare remole a terta visum nec uriquan thi nidificantem scalus, unde juste e J. zuna cerlusinasus.

-ob) 11ab. galuce inedias illeer Zalurlielidnnts ef larng

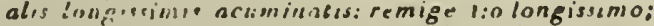

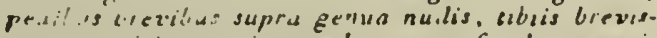

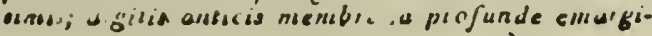


I. caspia, (hieme): rostro robusto coccineo, fronte parteque vẹrticis albis ${ }_{2}$ occipite nigro-alboque variegato, nucha corporeque.stopra canescentibus; subtıs alba; pedibus nigris. (astace): pileo toto atio.

Linn S. N. ed. Gmel. 6o5. 8. Sparrm. Mus.62. Ödmann. in Act. Holm. 1782, 2ño. Retz. Fn. 126. Nilss. Orn. 209. Sierna. - Sv. Zool.

Sv. Caspisk Tär $n$ a.

Junior: similis adulta in hieme, sed supracinereo-brunnea: maculis tasciólisque nigricantibus; cauda ad apicem remigibusquie nigricantibus; rostro opace-rubio apice nigricante.

Hab. rolitans in mari balthico, raro ad orns ejus. Idit pisces virenles, nidifical In rupibus nudis rol in arena marina el ponit ova $2-3$ grisescente-viridin: macn lis magnis brunneis et alris. - Congitudo ovis 20-2 poll. Tibiarum 16 poll. Iris lutescente-fusca. Remiges cinereobrunnei, cauda brevis clare cinerea.

2. cantiaca, (Tieme): rostro tenti nigro apice lutescente occipite nigro-alboque variegato; corpore supra canescente; fionte vertireque subtus albis; pedibus ńigris.

Linn. S. N. ed. Gmel. Go6. 15. Steran. - Nilss. Orn. 2rr. Sterna canescenc. Sע. Zoob.

(astate): pileo pennisque longis occipitis atris, collo antico pectoreyue roseo-albis. Linn. S. N. ed. Gmel. 665. 12 Sierna africana.

noca unitis, postico libero; unguibus brevibiss ar. cuatis; cauda plus minusve furcatr. Quum nom. gener. omni derivatione cares, nisi e nomine Suecano formalum; gracum X\&ledw, Hirundo morino, conscrvevimus.
Sv. Kentsk Tälna.

Junior: colore albo nigroque mbiescenteimmixtis; corpore supra albido-ruber fuscio. lis fuscis, scapularium marginibus fuscis, apicibus tectricum alarum semicirculo termina. tis; pennis secuindariis et remigibus nigro-cinereis albo-narginatis; subtus alba; rostro livide-nigio: apice lutescente; rectricibus $\mathrm{ni}$ gricantibus: basi cinereo apiceque albo.

Linn. S. N. ed. Gmel. Gog. 24 Sierna slriata. Retz. Fa. 127. Sporrin. Mus. 65. Sterna nubilosa.

Hab. Gottlandize rarius, ipse, passim etjam in insulis aliis balthicis, $D$. Thunberg; in Norvegia sepius presertim ad orus maris seprentrionalis. Vescitur piscibus. nidifical in arena marima 1 , in rupibus nudis et ora $2-5$ alba maculis nigricantibus ponit. - Longitudo 15.16 poll. tibiarum 10.lin. Rostrum longum. Frons et rertex alba, ad occipul maculis minulissimis nigris medio pannarum; pennis longis occipitis at is albo-fimbriatis; cruce nicricante ante oculos. Iris nigricans. Dorsum, scapulases et rectrices alarum canescentes Remiges cinerei $m=r g i n e$ interiore late albi. Pedes nigri: tubis, poll. jongis palmibus subius ocliraceis. Cuuda longa vulde furcata.

3. Dougalli: rostro toto nigro; pedihus aurantiacis cauda alis longiore; (astate) alba: capillo atro; corpore styra clare cinerco; pectore rosen tincto.

Temminck Manuel d'Orbibologie Tom. 2. p. 738. Sierna $-S v$. zool.

Sv. Dougalis Tärna.

Junior: subtus alba: rostro nigro; pedibus lutescentibus.

Hab. in Norvegia, teste D. Temminrk. Habitus hiemalis et occouonia ejus incopnita. - Losgitudo 15 poll. Rostrum longuar, gracile. Veriex nucliaque aler. Doi 
um, jcapulares et aix ciare-cineres. Co!lani el sulblis albuns. Renuiges cincip margine inleriure albi, sell mase kine remigis primi exleriure nigro. Cauda alba: pennis felpralibus extimis longissımis subulatis, alis 2 poll. Ioncior. Pedes anranliaci: tibis 9 lin. longis: unguibus nigris brevibus, infenuedio tubia breviore.

4. Hirundu: *) rosilo mbro, apice interdum niçro; pedibus rubris; pileo atro; cor. pore supura cano, sublites albo pectore cancscente-lincto; rectricibus extimis extus nigrofin.ris.

Linut S. N. 229. 2 Fn. 158. Rete. Fa. 124. Hamm. Fn. 169. Nilss. Oı1. 2ro. Sierna. - Sv, Zool. $S_{2}$. S vol 'I i ina. - N. 'Terne, Hattetarne, Hietlingterne, Sandtzerne.

Junior: rostro basi aurantiaco, fronte sordide-alba, vertice occipiteyue albo-imnixtis pennis longis, nigrofuscis inargine extimo apiceque albidis, peunis corporis supernis leviter albrimarginatis et terminatis maculis irregularitus fuscis I. rubrescentibus; subtus sordide-alba; rectricihus cinernscentibus a picibıs albidis; pelibus anrantiucis.

Hab. frequellussima in lora Scandinavia, inlerdunt aijom ad ayua dulces prino reread oulusunum, quum

-) Coleb. D. Temminck Suernam arcticsm: rosiro gracili obscure ulivarmerubio apices houd nigro; longi. tudine tibiarull $\dot{0}$ poll. ceudaque valde furcala elis

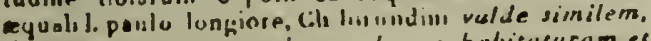
descripsit. intsa carrulan pularem habisusuram et

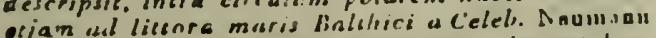
cussom. Re veru culem uumquarn visa est hiec cupsom. Re veru aulem inmquain visa est hrec adnumerandu. -mpigral. De, 2-4 Puseo-oliegcea meculis numernsis rine. reis el nigris poulit, ut cenfunceres lies id incubans. Fi. clilat pisciculis rivis l. morrins, sepe fliam insucliv a jll9ricis. Timidus extra jal,ictilm se suslelltat, crian : t rerk kreck et friehö h! - Longitulo 53.14 pill liti ri . brunnea. Pilous el paunr. occipitis longm atri. Henizen cani apicibus brunnescentibus. Cauda ro'd forficala cilo. alis requalis 1. brevior. Pedes inngiendine libiarum, rolia.

5. minute: instro aurantio-luteo apice ni. gro; pileo. Iorargue nizris; corpnye supra pallide cann, subtor, fronte, sinferciliis, candaque albis; remighus 3 atimis extus fuscis.

Linn. S. N. 228. 4. Nilss. Orn. 213. Sterna. - Sv. Zool. Siv. Lill Tärna.

Jun. fionte lutescente-alba, pileo brunneo, lineolis nigricantibus, macula ante et pone oculos nigra; corpore supra lutescente-biunneo pennis nimricante-cinereo marginatis, remigibus rectricibusque lutescente-albo terınipatis.

Hab. cum cnngeneribus passim Goulandio Jpse, Forvegia el Svecise australis D. Nilsson. Inserta slats. rermiculi marini et ova piscium, rare pisciculi cibus ejas sunt. Ora 2-3 clare ríridescentia maculis magnis bruaneis et cinereis in littore inter conchylia $l$. in arena ponir. - Longiludo $8 \frac{3}{2}$ poll. Iris nigra. Peclus canescens. Cauda valdo forficata: uropygio albo. Pedes aurantiolutei.

6. nigre: rostro nigro, capite atro, corpore supira cano subtus nigro-cinereo, crisso albo, pedibıs brumnis 1. nigro-purpureis.

Linn. S. N. 227. 5. Fn. I59. Retr. Fn. 125. Hamm. Pn. 170. Nilss. Oru. 212. Slerna. - Linn. S. N. 228.7. Eierna Gssipes. Linn. S. N. Ad. Gmel. 608.90. Stern. obscura. Sv. Zool. 

Sv. svart Tärna. $-N$. Sort Tàne, Sösvale, Sort Kirre.

astace: fronte, regione inter rostrum et oculos, gula, colloque antico nigro-cinereis.

Junior: fronte, regione inter rostrum oculosque, collo antico, crissocuse albis; macula laterum pectoris naguanigricante cinerea ante oculos adscendente; pilco nigro, pennis dorsi scapularumque brunnescentilus rubrescentealbo marginatis et terminatis; alis, uropygio caudaque cinereis tectricibus rubrescente-terminatis; basi rostri irideque brunseis; pedi. bus livide-brunneis.

Linn. S. N. 22s. 5. Sierna nevia. Retz. Fn. p. 163. descript. sub Sterna hirundine.

Hab. non infiequens. Nidificat inler gramina lillora. lia rel in foliis Nymphere ora 2 -4 clareoliracea maculis numerosis fuscis nigrisque circulum lalum vedio formanlibus ponens. Viclilat insectis rermiculisque marinis.-Lonniludo gt poll. Iris brannea. Colluni postice atrum. Frons, regio iner rostrum et oculos. gula, collum anlicum ad peclus alba. Uropy grium et rectrices carrulescential. plum. bea. Remiges a primores exlus albo-margiaat. Cauda parum furcala. Tibize $7.8 \mathrm{lin}$. longze.

\section{G. 92. LARUS '), Sv. Máse.}

8. Marinus, (hieme): albus: dorso alis. que atris; rennigibus ad apicem album atris;

-) Hab. gen. Slatura fore columba, sed collo, pedilus alisque longioribus et alis aculio.itus. Alre longre acuminate: remige 1:0 longitudine fere secundi: cruda equalis, brevis; pedes graciles, supra genu nudi. Nom. Ger. Latinum rostro albicante-luteo, angulo igneo; pedibus allidis.

(astato) albus: capite immaculato, circulo oculorum nudo aurantiaco, dor'so alisque nigris. Linn. S. N. 225. 6. Fn. 155. It. Gotll. 224. It. Vesirogoth. 136. - Odm in Acr. Holm. 1783. ror. el Retz. Pn. Ir7. (pedibus rubris?) Nalss. Ora. 214. Hamm. Fn. 62. - Su. Zool.

Sv. Ha fs Måse, Hafmika, Hoınåkn, Hafstrut, Svarttrut. - N. Svartbak, Havmaase. L. Gairo.

(biennis) dorso alisque nigris: brunneo grie seoque maculatis; cetero albus: maculis par. vis brunneis griseisque sparsis; cauda nigromarmorata; rostro livide.Jutco, nigro-maculato, macula media anguli iguei nigra.

Zinn: S. N. 225. 5. Larus nerins.

(annuosus) medio plumarum et pennarum nigricante et brunıeo minus extenso, margine albidiore circumdato; capite fere albo; apice basique irostri lividis.

(magis jun.) capite colloque antico macu. lis numerosis brunneis, inferioribus majoribus; supra pennis rubrescente-albis medin nigrobiunneo; margine rubrescente-albo tectricum alarum transversali fasciformi; subtus sordide griseus, maculis striisque undulatis brunneis; remigibus nigricantibus albo-terminatis; rectricibus mediis plus nigris quam albis, lateratibus vero ad apicem nigris; rostro atro; iride circulorque oculorum nudo brunneis; pedibus livide-biunneis.

2. Hob. in mere as frequeas, ubi piscibus, cedaror: 

bus variis, eljam pullis aliarum avium, rariug conchylis victitat. In rupibus litloralibus nidifical et uva $\mathbf{3 . 4}$ obscure viridi-olivacea maculis plus minusve magnis punit, interdum ter per annum, primo nedio mensis Maji; clamat warag et $g$ u iovis! facile mansuesceus non'ineptus piscetur, imprimis in gemeratione a:a, ancundum opinionen $D: i$ Ódman, erit. - Lougitudo m. 25-27 poll., $f$. 24-25 poll. Iris clare lutea brunneo-mumnrasa; cirfulo oculorum nudo rubro. Penna capitis alba: niedio clace brunneo-striala, sed frontis, colll, et subtus dorsi inferioris caudaque tolze albe Duisum ou!erius, scapulares - Ixque atra carolen-tincta: ajicibus remigum, pennarum s-undariorum el scapularium albis. Pedes albidi: longitudine ribiarum a poll. 5-6 lin.

2. fuscus, (hierna): albus: dorso alisque nigris, remigibus 2 extimis nacula ante apicein ovali alba; rostro litleo, angulo igneo; pedibus Havis.

(coscase): capite immacilato.

Linn. S. N. 225. 7. Fn 154. Retz. Fin. 188. Ölm. in Aet. Holm. 1783. 105. Nilss Or11. 216. Hlamin. Fn. 26tSข. Zool.

Sv. grảbrun Máse, Silluıase, Sillmåka, Trutt, Linse. - N. Öimor, Eyınor, E' mor, Gulfötting, Sildmaase. - L. Sobmor.

Junior: supra el alarum pennis nigro-fuscis lutescente-marginatis; lrmigibus onuibus. nigris inmoculatis; rectricibus basi clare griseis nigronmarmoratis, medio hlris, apice al. bis ; rostro nigro basi brunnesceute; pedibus. sordide ochraceis.

Hab. in mare non infrequeasimprimis seplentrionali. Pisces cibus ejus prsecipuus est. In arella littorali $]_{\text {. in }}$ supibus djascentibus ora 2.3 fosce maculis nigris ad Enoin Maji I. initio Junii posit. - Longitudo m. 19-20 poll." f. $18-39$ poll, Iris clase lutea; circulo codorum tudo robro. Penar capitis collique in lateribas albx: medio clare brunneo-strialze, sed tronlis, colli antici el subius, dorsi inferiuris caudxque tole albx. Dorsum superius. - capulares, alzeque alra cinereo-tincia: remigibus 3:0 et crteris cum pennis secundariis el scapulusibus tenuissime albo-rerminacis. Pedes Aari: longitadine tibiarum, poll. x.2 lin. ,

3. glaucus, (astrute): albus: dorso alisque canis remigilus apice albis; rostro luteo, angulo ignen; pedibus lividis

Odm. in Act. Holm. 1783.97 . Retz. Pn. $\$ 2$ Brünn. Ora. 148. Niles. Orn. 215. Hamm. Pn. $163-S v . Z 0 o b$. Sv. bl ágr ii II ås c, Gråtrut. - N. Blaamaa. ge, Granmaage; Sildnaage.

Junior: sinilis junioribus $\boldsymbol{L}$. marini sed ro. stro lougince et validiore, rachibus remigun albis (in.his nigris), colore griseo brunneoque pallidiore; remigibus et pennis secundariis pallide fiscis.

f'or. B. tota pallide-grisea fuscescentenebulosa el variegrata. Nilss, I. c.

Hab. in Norregia at in rupe nuda in mare extra Holmiom, Kolken dictn, D. Odmann, ubi nidulatur, ora -3 viridescentia maculis sparsis nigris, ad extremitatem parvam elongata ponens. Piscibus, aviculis, cadareribus, excrementis nammalium marioum victital. Clamal $\& y c-$ kyckyck el iritalus $g$ io $u w$ ! - Longitudo 26 poll. Iris Iutea : circul,s oculorum nudo rubrn, Caput, collum, subifs raches et plus a poll. exiremitales remiguin, epices pennarum alerum ceterum, csodeque alba. Dorsum superius et alz clar corulescente-cineres. Pedes liridı; longitudine vibidrum a poll. da-11 lin.

- 4. canns, (hieme): albus: rapite lateribusque colli fusco-maculatis; dorso alisque conis; remigibus 2 extimis macula ad apicen 

alba ; rostro basi viridi-cæruleo, apice ochrareo; pedibus canis lútescente-maculatis.

Nejer Taschenbuch Deulschlands. Vol. 8. p. 480. L. cysnorliynchus.

(astabe): capite colloque imnaculatis; rostro ochraceo; circulo oculorum nudo cinna. barino; pedibus clare ochraccis capescentemaculatis.

Lınn. S. N. 224. 3. Fn. 153. Retz. Fn. 119. Ödm. in Act. Holm. 1783. 109. Nilss. Orn. 213. Hamm. Fa. 160.Sv. Zuol.

Sv. hvitgrà Mảse, Fiskmåse, Fiskmåka, Homåka, Mave. - N. Sxunğ, Sxing. L. Straule.

(biennis): fascia caudx fuscescente ad apicenı, rostrique medio nigro-fusca.

(annuosus): capite albo fusco-striato, basi rostri cæruleo-cinerea; dorso curuleo-cinereo tincto, pennis intermixtis fuscis lulescente marginatis; subtus albidior.

Jun. rostro nigro basi livido, et nigredine ante oculos, quorum circulus andus fuserns; capite colloque supia fusco-griseis, ut etjain pennis dorsi alarumque, sed lutescente-1. rubrescente-marginatis, remigilus caudaque ni-. gro-fuscis; gula ventreque basi apiceque caudie allis; froute et subtus albida maculis grisescentibus; pedibus latescentibus.

Linn. S. N. ed. Omel. 596 . 13. L. hybernus.

Ifab. ad littora maris et Incuum hiviorumque rel-: gatissimus. Pisces, insecta vermesque matina conchylia bivalvia cibus ejus sune. Venienies gregatim ad cerrain, procellam iudicant somanter anxie et clamose vi ie, vi ie,'

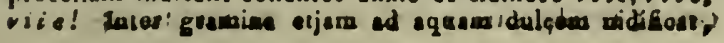

ora 5 obscure viridis, nigromaculats ponens. - Longiindo 16.17 poll. Kosirum parrum: ore infus aurentiacb. Iris fuscescans: circulo oculorum nudo rubrescenle-bronneo. Apices pennerum scapularium secundariorum et renigum albi. Tibixe longitudine 2 poll.

'5. tridlaotylus, *) (hieme): albus: capite lateribusque colli, dorso alisque canis; remigibus extimis apice uigris; rostro viride-luteo; digito postico verruciformi, mutico.

Miey. Taschenb. 2. 486 -

(erstace): capite collique albis.

Linn. S. N. 224. I. Brünn. Orn. 140. L. Rissa. Ōdm. in Act. Holm. 1783 . 113. Retz. Fn, 115. Nilss. Orn. 219. Hamm. Fn. 164. - Sv. Zool.

Sv. tret â a d M a se.-N. Krykie. - Z. Skarro. (annuosus): albus: dorso cano pennis fusco-maculatis mixtis; maculis nigris ante et pone oculos pemnisque ad aures et in nucha in obscure-cana transitis, sed macirlis alarum nigris fuscisque; rostıo viride-luteo nigricante matulato.

Linn. S. N. 224. 2. Fn. $15 \%$.

Junior: allidis: unacula ante oculos, in regione aurium nigricante-caua, in occipite muchaque uigricante; dorso alisque obscure-canis nigro-fusco terminatis, margine superiure alarum nimro, pennis scapularibus et secun. dariis maculis magnis nigricantibus, remigibus nigris; caurla ad apicem nigra, albo-terninata et extus alba; rostro, iride circuluque oculo. ruin, nude nigris.

Hab. in insulis Bahusix Norregireque non iofrequens

-) Nomen specificum Rissa, islandicum; unde inepum. 
Pisces, ora eoran, insecteque marine riclu, ejas suat.

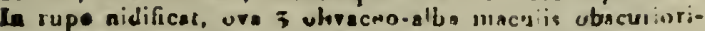
bus numerosis atque cineres sparsis pnnit. Clamat: $k$ ul geghef. - Longiludo is poll. Rostrum parrum: angulo rix conspicuo. Os.er cesculus oculorum rufus. li fusca. Caput ex pars lateralis colli cana, strinlie nigris ante oculos. Frons, regio iculerum, curpus subtil, i11opygium caudaqne alba. Alze caux: renige extimo 10 to margine 2-4 apice niksis. 4:0 macula minulissima alb.x, 6:0 foscia ante apiceın altuin nitrs. Pedes fusci et ob. ecure olivacei: tibiarum lungitidine poll. 4.lin.

6. alurneus: albissitums: rostro ocluacen, basi canescente, pedibu: nigisis.

Linn. S. N. ed. Gincl. 5,6. I4 Nilss. Oin 21\% Ödm. in Act Holm. Ir8j. tou. L nireus. Ilamm Fn 168. L. albus. - Sv. Znol.

Sv. elfe ubeuslivit. M is se. - $N$. Valdmaase, Heynaase, Merlentiase, Malal, Manafiar.

Hab. Norvegia ad mure plaviale, rarissme àd littora Srecia Piscibns el cadoverbus vicrital -. Lungitudn is19 poll. Rostram magnum, viilum lris brunneo. Pedes *iri, longeitudine nbarum ' pull 5 l.n.

7. ridihundus, ) (hisurs): albus: inacula nigra ante oculos et nigricitule ad antes, torsu cann; rostro pediburque clare-cinnabarinis.

Linn. S N. 224. 4. I cinerarills.

(ascale) capite colloque superiòre nigrofuscis: superciliis albis; rostro pedibusque pmr. purascentibus.

Zinn. S. N. 225 .9. $\delta$ im. I. c. 120. 9. Retz. Fn. 120. Nilss. Orn 200. - Sv. Zool.

Sv. Skrat $\mathrm{Ml}$ is se.

(annunsus): docisn cano pennis fuscescentibus alisque canis pennis fusco-maculatis et

-) I alricilla Rel» minime in Scendinevio viso, sed incolo Burepo ewsralis est. !ntescente marginatis immixtis; capite alloo cireren-niacoulalu, rritcular fuscaa ante oculns et ad anre; basi rnstri rubrescente, apice fusco. Linn. 5. N. ed. Gmel 597. 15. L erythropos.

Junior: (:is)itr crecipilrque clare finscis, macula ollı pune roulu: fiscia unchali et sub: tus albu, abedinc remiceo-tincla in collo aitico et hypochnodpiis, quibms fisco-cruciatis; dorso pennisque scapularibus tectricibusque inediis nigro-luscis lutercente-marginatis; tectricibus majuribus anis; superiore alarum parte, "ropysio candayue albis: rectricum apicibus nigro-fusci-; remigibus, albis, extus apiceque nigris; basi rusir livida, apice nigro; pedibus fulcicentibus.

llab. a aljuas lam salses priam dulces minus freo guens, ubvias prasertim in Golli, nlia ipse el scania $D$. Retzius. Victitat piscirnlis, oris lisclum insectis verm:busque elian teltestribus. Nitificat in littoribus gramicosis ova 3 nbscureolivocea maculis fuscis nigrisque sparsis ponens. Ciamal sonu furriente: irre-kreck reck! - Lonpiruda in-15 pull Iris nigro-linsca. Capul, collusa caudanue ribs macila nigra ante uculos, pigricante ad aures; collum inteicius, peclus, abdoinen cissumijue alba leviler roseu-tincta. Dorsum alaque cans, alis intus nigricante cillereis, matgine exteriore remigibusque albis: remige prono margine extus tolo pl dinidio intus cum apice nigris, ipso apice tamens seniorum iaterdum wha Longitudo tibiarum a poll 89 lill.

8. menusus, (hiemej: albus: rostro nigro. fusco; capite: supra cincreo-nigro: dorso alis. que canis; pedibus clare cimabarinis.

(restace) capite tolo culloyus sur)eriore nigits, macula albs pone venlwi; culfo inferiore et subtus alloo rufo-tiuctr); uroj) bio cauda-

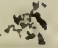


que albis, remigibus pennisque secundariis albo terminatis; rostroobsure-purpureo; iride obscule-fusca; pedibus chermesinis.

Linn. S. N. ed. Smel. 595. 12. Öllm. in Acl. Holm. y 783. 121. 10. Repz. Fp, addead., 273. Nilss. Orn. 221. Sv. Zool.

Sv. Liten Måse.

Junior: rostio nigro-fusco; fronte, regione oculis, subtuis et $\frac{2}{3}$ caudx albis; vertice occipiteque nigro-cinereis; nucha dissoque fuscogriseis; tectricibus alarum minoribus albidis nigricante griseoque maculatis, mediis nigrogriseis brunnescente-marginatis, majoribus extus apiceque albidis; remigibus $1-4$ extus apiceque nigris intus albis, $5-7$ extus cinereis a. pice albis; cauda subfurcata fascia apiçali lata nigra, extus angustiore; pedibus incarnatis,

Hab. in Gotelandia rarissimus, D. Excell. Vir. Com. Troile Wachemeiscer el ipse. Insectis verm:busque mat rinis rescitur. - Longitudo so poll. 2 lin. Ir is nigro-fusca. Froos, regio inter rostrum et oculos macula pone eos, magna, gula, sublus caudaque aba. Orificium anris cine reo-nigrum, celero lolus supra clare canus. Reniges lale albo-lerminalí, rachubus fuscis. Alre inlus nigriconlea. Longitudo tibiarum ix lin: digilo postico brevissimo, unt lue parum conspicoo recto.

\section{G. 33. LESTRIS *), Sv. Rofmåse.}

-) Habir gener. precedentis, \& quo tamen moribus et characseribus indicatis distinctus, ut etjam alis mediocribus: remige 1:0 longissimo; cauda sub. rotundala: rectricibus 2 mediis ceteris longiorilus; tiluiis lorgioribus: digitis anticis palmatis, postico brevissimo, unguibus magnis rurvatioribus. Nom gen. - Ayspls, pradotrix derivatura.
.1 1. oasaractes: capite regioneque oculorum uigra, fuscio; dorsa, iciapula rilınsque rubris; rostro pedibusque atris; rectricibus mediis 2 finearibus, apice rotundatis.

Linn. Ss. N. 226. 11. Larus. - Brünn. Orno 125. Reiz. Fa .225. Cararacla Skua. Su Zool.

Sv. For's R ofmåse. - N. Skue, Kavörn.

\%ab. Norvegise, ad mare glaciale et septentrionale. Piscibur, ariculis, ovis avium, molluscis rariis, pxcremeniis mammalium masinorum victital. Ares caplat, aquilam ipsom persequens; interdum etjom agnos aggreditor te diripit. Nidificat in graminosis el frulicesis montinm allium ora $3: 4$ acurissima oliraces maculis magnis Ruscis sparsis ponens. - Longitudo 20-21 poll. Rosirum basi et iris fusca. Collum er tota s vis subtus rabrescenle-griseam in brunneo rergens. Dorsum el scapulares palliderubra, pennis nigro-fusco-marginalis. Tealrices alarum culdaque et pennx secundarize fusce; remiges ad mediunt'ysque albi, cetero nigro-fusci: barba alba. Recirices abx. Longitudo tibiarum? poll. 6-7 lin.

2. pomarinus: capite, dorso alis candaquàe higro-fuscis; rostro olivaceo, apice nigro; 1)dibus atris; rectricilus 2 mediis lincaribus, apice volnndatis.

Témminck Manuel d'Ornitbologie ed. 2 Tom.2.p.79j.Meyer Vüg. Deutschl. vol. 2. Heft. 21. Gg. vara. Larus parasilicus. - Sv. Zool.

Sy. Fisk Rutión â.

Junior: nigro-fuscus: pennis colli nucheque longioribirs et subulatis, paulo splendidioribus, lutescente-brunneis; rectricıbus 2 mediis linearibus ad apicem rotundatis, sed brevioribus quain adultiurum.

magis jun. capite colloque pallide fuscis , colore clariore variegatis, ante oculos regione 

nigra; dorso, scapularibris' et 'tecticibus ala. rum nigrn-fuscis: jennis rubro-ernciatis; pos ctore et fiypochondriis cinereo-brinneis ma. culis lineisque flexnosis transversis mbris; ab. dominc,urnpygin leclicibusqne coudø supra el sublus.nigin-rubroque late-fasciatis; rostro bnsi viricte-cxruleo, apice nigio; pedibus $\$ x$. rulen-cinereis: digilis membranxque basi cum ungue postico.altbidis, celero nigris.

Haho imprimis Norvegin in Ira circulum arclicum, raro Srecix. Piscibus, (quos Laros persequens demillert cogis,) collaver bua et, oris avium rescilur. Nidulalur juler gramina muscosyae montius in locis marecosis, ubi ora 2.3 mina miscosyar montiun in macilis sparsis nipricantibus aculissina - longiludo $15-16$ poll. Iris lulescente brunnas. Pun colli es nuche louga subulate, spleadide antooPeine calli el nucho lollar subulate, spinadide alleafuscie in raunis macul, fuscia. Hypocbonduse ol lecirices ceude illferinres iransperse fuscu-macularze. Tibize roIcursum asperale longitudine 1 poll. is lia.

3. purnsiticus: capite supra nigro-fusco; fronte, collo subtusquc albis; dorso, alis cisldacye obvente cintereo-brunneis, apicibus pennarum nigricantibus; rectricibus inediis longissimis acutis. .

Linn. S. N. 2.26 10. Pn. 156 . H.1mm Fn. 168 Larirs. Retz. CI1. 122. Brünn. Orn. 229. Calalacla. - Nilss. Orn. 2n. - Sv. Zool.

Sv. Suylt Rofinảs c, Struntjazer, Svartlisqe, Jublben, Elof. - N. Strundjagre, Tiulvi, Tyvje, Jutyv; Jon Tuifra, Jubni, hive, Kivin.

iunior: s"y)a cinereo-fuscus, sublus palli. divi;

\section{secundum}

Genera:

Dizisio s. pedibus didactylis : \&. Strathio.

Diva 2. ped. 5-daclylis: 2. Gauria B. Thea Brisson), 3. Thresys B. ot 4 Cbelarga B. (Casuarius Briss,

Div. I pedibus colligatis: 1 . Otis, 2. Obdicprienas Cuvier, 5. Cursorius Latham, 4.Syrrhaptes Illiger. Div. 2. pedibis gradariis: 5. Ortyx Xenocrates (Orlygis Ill.)

Div. .. libiis nudis : 1. Perdix Briss. 2. Cryplonyx Temminck.

Div. 2. tibiis plumosis: 3. Crypturus III. 4. Pterocles Temm. 5. Tetrev.

4 . "Papidistus vieass.

I Edolius Cuv. 2. Tyrannus Briss. 5. Muscipeta Cuv 4 Muscicapa, 5. Gymnocephalus Geofroy. 6. Ceplmalopierus Geofir: ? Gyinnoderes Geoffr. 8. Ampelis. Q. Ceblenyris Cuv. Ampelis Temm. 10. Casmatiynclus Temm 11. Procuias 1ll. lissimo:

t. Caprimulgus, 2. Cypselus 11l. 3. Hirundo.

longalo:

1. Uxyrhynchus Temm. 2. Sizta, 5. Orthongx. Temm. 4. Dendrocolaptes Heirnann. S. Anecom rliamphus B. (Xenops Hoffmig.) ô Anabales Ternm. 7. Opelioshynchus Temm. \& Cerlhia, 9. Climsclejis Teinm. in.. 'I ichordrin:a Jil. ix. Upupa, I2 Epimachus Cuv. 13. Diepanis Termm. 24. Diczum AElianus.

I. Meliphag Levin (Philodon Cuv.) 2. Nectarinis Ill. (Careba $T(\mathrm{~mm}$ ' 3. Cinnyra Cuv. A. Irnchilus, 5 Mellisuga Briss. (Orbhorhynchua Lacipéde'.

rais fere longiundina inı unilis:

T. Mermps, Alcedo, 5. Capy: B. (Alcedo Cuv) A A reinles B. (Dacello Learh.) 5 Todus. x. Prionises 1:!. 2. Abucercs B. (Buceros Cuvo) 
nigra; disiso; scapula ribus' et 'tecticicibus ala. rim nigro-finscis: pennis rubro-ernciatis; pow ctore et hypochonthiis cinereo-bivnneis maculis lineinque flexuosis transveosis mbris; ab. domini, surnpygin lectricibusqne caudeo supra ef suburs nigrn-rubroque late-fasciatis; rostro basi viricle-cxruleo, apice nigio; pedilus rulen-cinereis: digitis menbranłeque basi cun ungue postico altidis, cetero nigris.

Hal. imprinis Norvepive inara circulum arclicum, raro Srecix. liscibus, (quus Laros persequens deminere cogit,) callaverbua et, oris avium rescirur. Nidulatur iuler gramina muscosyau monlium in. locis marecosis, ubi ora 2.3 aculissina cineieo-olivacea maculis sparsis nigricantibus l.nult. - langitindo is-16 poll. Iris lulescente brunnea. Penne colli et nuche lougre subulata, spleudide aureolutra. Guls, collun anticuin, peclus et obdqmen albs: foscia inrquala maculis fuscia. Hypocbonduie el tecirice caude inferiores iransperse fuscu-maculaix. Tibize to trursum asperale longiludine I poll. Ir lio.

3. porasiticris: capite supra nigrn-fusco; fronle, collo sublusquc albis; dorso, alis cilldingue obure cinereo-brumneis, apicibus pellnorum uigricantibus; rectricibus inediis longissimis acutis. .

Linn. S. N. 226 to. Pn. 156 . H,Jmm Fn. 168 Larist. Retz. Tu. 122. Brünn. Orn. 127. Cataracia. - Nilss. Orn. 2 n. - Sv. Zoul.

Sv. Suylt Rofinàse, Struntjager, Svartlisise, Jublen, Elof. - N. Strundjagrr, Tiulvis, Tyvjo, Jotyv, Jon Tuifra, Jubon, hive, livin.

iurior: supra cinereo-fuscus, subtus pallidiur;

\section{secundum}

\section{Genera:}

Divisio r. pedibus didectylis: . Straubio.

Diva 9. ped. 3-daclylis: 3. Gauria B. (Rhee Brisson), 5. Thrasys B. ol 4. Chelarga B. (Casuarius Briss)

Div. I pedibus colligalis: 1. Olis, 2. Obdicprienus Cuvier, 3. Cursorius Latham, 4. Syrithaples Illiger. Div. 2. pedibius gradariis: 5. Oriyx Xenocrales (Ortygis Ill.)

Div. I. Libiis nudis : 1. Perdix Briss. 2. Cryplonyx Temminck.

Div. 3. ribiis plumosis: 3. Crypturus III. 4. Pterocles Temm. 5. Tetrav.

44. "ropidristus vaess.

1 Edolius Cuv. 2. Trrannus Briss. 5. Muscipera Cisv 4 Muscicapa, 5. Gyinnocephalus Geoffroy. 6. Ceplalopierus Geoffr. ? Gyimnoderes Geoffr. 8.Ampelis, g. Ceblenyris Cuv. 'Ampelis Temm. ro. Casmarliynchus Temm 18. Procilias 111 . lissimo:

I. Caprimulgus , 2. Cỵselus 11l. 5. Hirundo. longaro:

J. Uxyrhvnchus Temm. 2. Silla, 5. Orlhonyx. Temm. 4. Desdrocolaptes Heirmann. S. Anecarliamplius B. (Xencps Hoffmsg.) 6 Anabalos Tenm. 7. Opeliurhynchus Temm \& Certhia, 9. Climaclesis Teinm. in. Iichesdram:a lil. 11. Upupa, 12. Epimarlous $C$ uv. J3. Diefunis Temm. 34.Dicxum AElianus.

1. Meliphag Levin (Philedon Cuv.) 2. Nec1arinia Ill. (Careba Tcmm' 3. Cinnyrs Cuv. h. Irochilus, 5 Mellisuga Briss. (Orshorhynchud Lacépedel.

prnis rere longiudine no uniris:

1. Mernps, 2 Alcedo, 5. Capya B. (Alcedo

Cuv) 4. Agruntes B. (Dacello Leisrls.) 5 Todiss. x. Prioniles l:L, 2. Abuceres D. (Buceros Cuvo) 5. Buterus. 
Subclasses, Ordines, Tribus, Nationes,

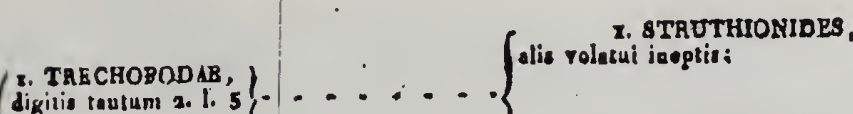

alis rolucilibas:

2. OTIDIDES

J. TETRAONIDES macule oculari nudn, alis rolatilibass 1. DIDIDEs,

(3. APSIAHAMIYHLE, costro Eorvicuto. cepite supra oculos nudo, alis rọtatilibas:

\section{NUMMIDAELDES,} regione oculorum plus minusro nuda; alis rolstilibus

\section{GALLIDES,} regione oculorum pluniosa; olis rolacilibus: Lefio 1. Astanacrrzan: digitis omnibus liberis?

8. APTL:RYGIOES

cers baei rosti plutuclatui alls volatui inoptis :

9. COLUMBAEIDES,

\section{(a)}

x. Peristera Ariscoceles, 2. Columbs, 5. Vinego

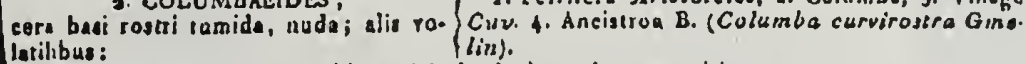
latilibus:

Divisio s. pedibos didectylis $1 x$. Strucbio. Div. a. ped. 3-dectylis: 2. Gapria B Throd Erisson), 3. Thirasys B. eI 4. Cbolarge B. (Cagu- Prius Briss)

Div. I pedibus colligatis: 2. Otis, 2, Obdicpriemus Cuvjer, 3. Careorius Latham, 4. Syrtheples Illiger. Dip. 2. pedibus graderiis: 5. Ortyx Tenocrales Ptygis Mis.)

Div. s. Lbii nudis: x. Perdix Iriss. 9. Cryptonyx Tomminck.

Div. a, tibiis plumosis: 5. Gryptaras IIl. 4. Pse. socles Tomin. 5. Tetrao.

2. Didus.

1. Pazudotaon 8. Cafaleogris Zinnes, 2. Penetoplie B. (Yenelupe Alerrenu), 3. Botyra B. (Ale. leagris L.J 4. Numide. 5 Wrex B. COurax Cu. viai) 6. Craxa B. (Crax Briss.), 7. Bromus B. (A'gue Temm.)

x. Pulyplecisus B. (Polyplectron Teum.) 2. Pa. vo, 5. Opisitocnmus Hoffinanssegg, 4. Lophorus Iemm, 5. Phasiantle, 6. Gallus Briss.

Log, 2. Tesmoacrtou: digitis allicis besi plus minusro unitis,

Color $x$. Intansaoszass: rostro plar usinguo integersimo:

x. GRORNITHES, forroribui fore tolis planosis; digitis plus un $\mathrm{i}$ Dusere besit.
2. ELAZOPODAR, digitis 3 enticis uno. que postico; suro dogua portico
5. CORVIOES,

rosiro robusto cultralo:

4. STUR NIDES.

sortro primatico I. cylindraceo:

5. RRINGIILAEIDES, rostro bravi conico, od basin iucresseto

6. ALAUD AEIDES,

costro olnugats conico:

(x. Corrus, 2. Cractes B. (Garrulus Briss.) I Coryocstactes Cuv. 4. AEgY Is l. ('I'omia V'aillant!) 5. Coraciss, 6. Colaris Cuv. 7. Grecula, 8. Pars disea, 9. Lainprotornis Taum.

1. Buphugs, a. Cassicus Cun. 3.Xanthornis Bris.

4. Sturnus, 5. Glauopis Forsier.

1. Loxis, 2. Psiltirusirs 2irmm. 5. Corgthu, $C_{u}$ 4. Pyrrbula Briss. 5. Pyrgita C.uv. 6. Pitylus. Cu 7. Prungilla, 8. Spernolege B. et 9. Canmabia B (Cordualis Cuvi) 30. Pliyrotome Asoline, 31. Co lins Arist. 32 Plozeys Cur. 33. Emberiza, 34. Pd. rus, is. AE gitisolus B. (Les Monstaches C'uv.) 16 Struthus B. (Les Reinis Cuv.)

8. Corydas B. el a Pseudocorys B. (Alaude Cuv:; Alunde.

apicom ovidenter emorginoto

X. Amthus Berlustein, 2. Motacilla, 5. decentor Bechss. 4. Phyllopsodsta Mloger, -5 . Rhadioa $B$.

7. Motacillarides, rostro subulatu:

Phyllopsewsta Mry.) 6 Regulus Rer (Sylvios Lasham) 7. Nannus H. (Troglndytes Cuv. 8. Titiza B (Calamodykes Mley.) o. Sylvis Latli. Io. Pheca B. (Ficedula Bechst.) \& 12. Saxicola Beehst.

8. LANIIDES 2. GONORHAMPHAB, rottro comprestiusculo apice currato: rosiro copoideu, plue minuste olopgato cyliadraceo,

9. TURDIDES, roatro compresso, apice vix curruto:

J. Leains, 2. Tumnophilas Vieiltos, 5. Yonge Vieill. 4. Pseris Cuv. 5. Sparectes Jll. si. Oxppio rus Cup. 7. Comeios B. (Criniger Tenm.) 8. Borita Cuv. 9. Groucalus Cuv. (Cellephyris Tomm.) ra. Becleylus Cuv. 1x. Eaphones Cuv. 12. Tona. gre, 25. Alieniphocelos $C u v$.

Dive z. digin externis orticulin 1:0 unitis: $r$. Molurus Pisill. 2. Py rrhocorax Cuv 3. Orinlus, 4. Misenura Sh 5. Myorbera 136 . 6. Conopoderas n. (Turdus Jongirostris Gmob.) \%. Turdus, 8. Ichis B. (Pastor Te inm.) g. Hydrichlo B. (Cinclus Bachse) 10. Bracliyurus Thunberz (Pista Temm.) Dive 2. digiris excernis lonfiludine nedis unitis; 12. Hapicola Cuv. 12. Phibalura Vieill. 13. Pipro. 114. Pardulatus Vicill.

10. AMPELIDIDES,

3. Edoliue Cuv. 2. Tyrannas Briss. 5. Muscipess Cuv 4 Muscicapa, 5. Gymnocoplisius Ceoffer 6. Cuplualopierus Geofir. ? Gyinnoderes Geoffr. 8. Ampelis, 9. Ceblopyris Cur. IAmpelis Temus. 8o. Casmarliynchus Temm sl. Procinins Ili. rostro dopresso, basi dilateto

Cob. 3. Promnostans : rietu sostri apoligeimos ix. HIRUNDINIDES rostro minntiusimo aubulato I. Caprimulgas, 2. Cyrsolus 3ll. 3. Hirundo. Cob. 4. Tryusaortras: rostro cenui blongato:

12. CURTHIAEIDÉS lingas modiocri beud oxtensibili:

T. Uxyrhynchus Temm, a. Sirta, 5. Orihony.s. Teinin. 4. Dendiocolapies Horrmann. S. Anecarhamphus B. (גenops Hoffinsg.) A Anibates Teinin. 7. Operiurhynchas J'renm. \& Cershis, g. Clima.

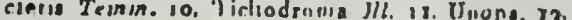
Epimachus Cav. 83. Droponis Tisam. Jf Dicxuni Eslianus.

15. TROC.IILLIDES lingus louge oxfonsibili: 1. Meliphage Levin (Philedor Cur.) 2. Necis. rinia 11\%. (Careba Temm.) g. Cinnyra Cuv. A Trrichilus 5. Mellisuse Briss. (Oriborhyncbu. luacipidai:

Log. 3. Hoposinosczisu| 14. MEROPIDES rostro integ tritimo:is. PRIONITIDES digitis excernis Pere longitadinn rote upli is:

(X. Merops? Alcedn. 35 Copj' B. (Alieds Cur.) 4. Agreules D. (Dacallo Learh.) 5 Tindus. 2. Ftinutes III, 2. Abuceros' B. (Daseros Cur 


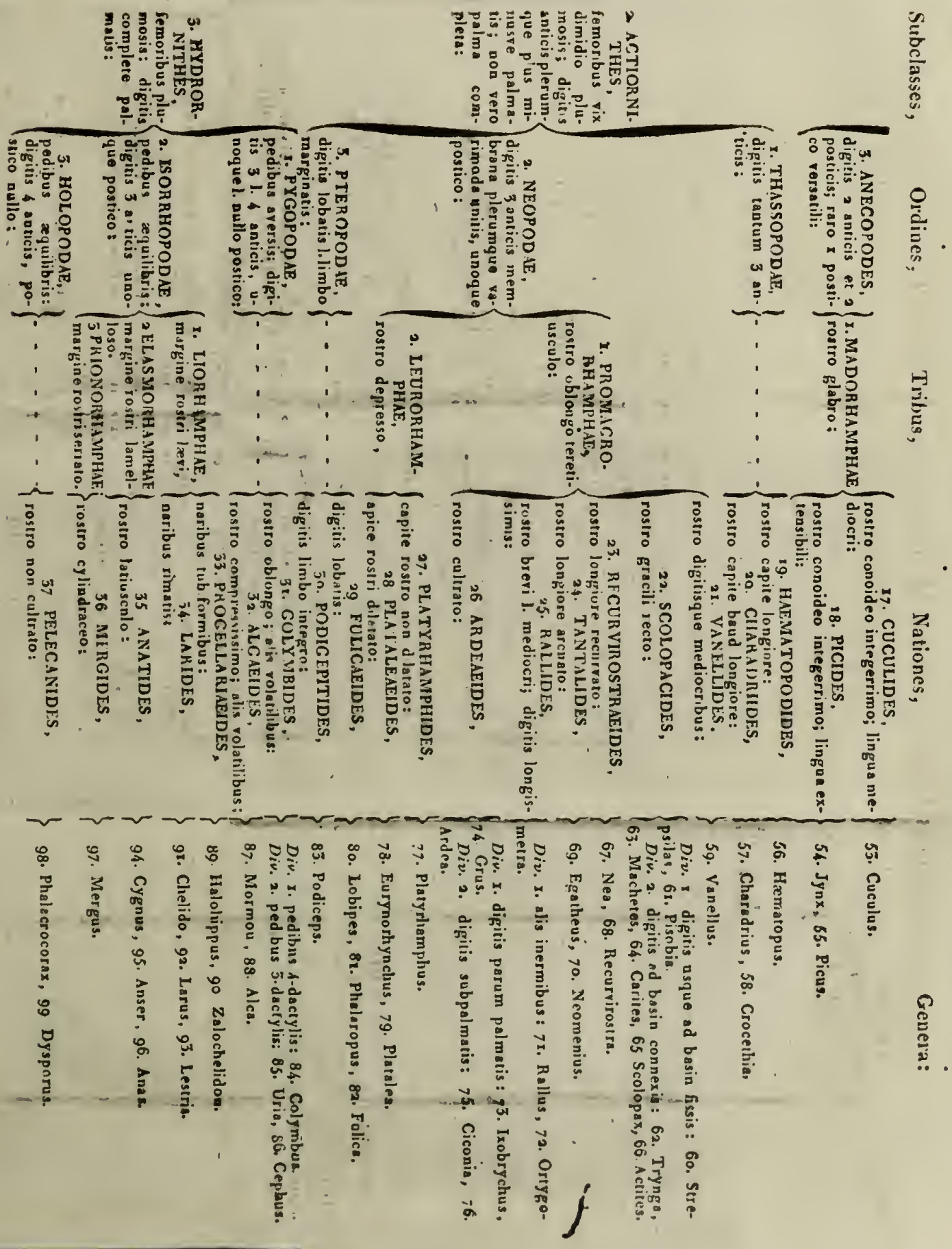



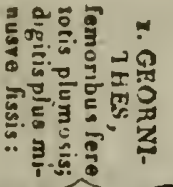

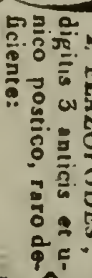

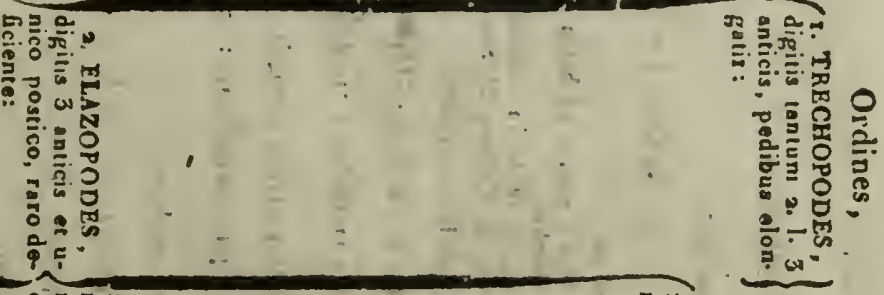

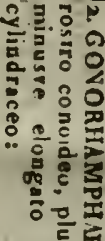
- E.

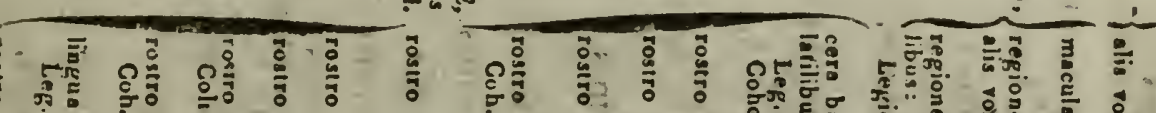

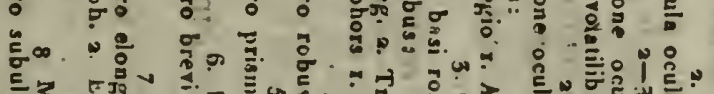

:

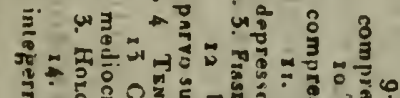

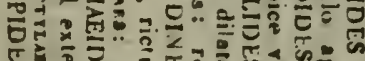

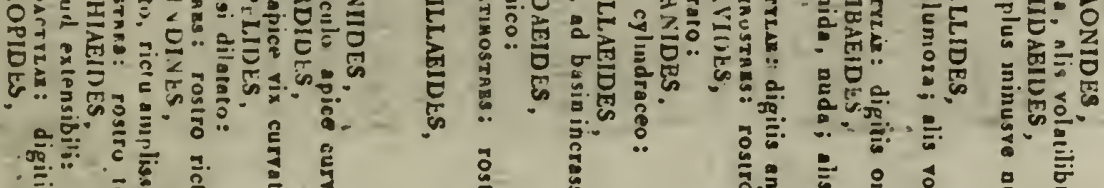

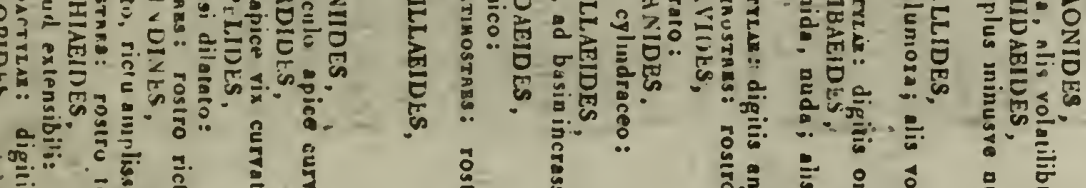

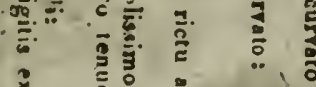

\section{(1)}

-

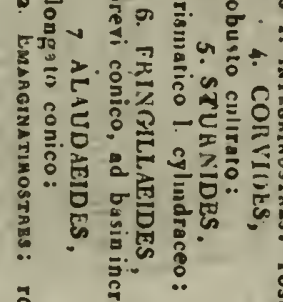

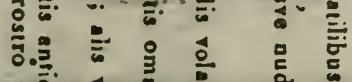
공

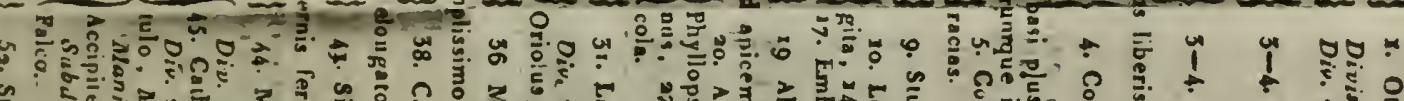

年

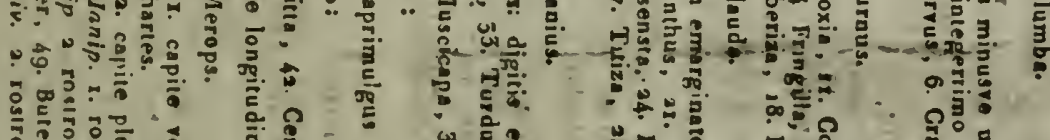

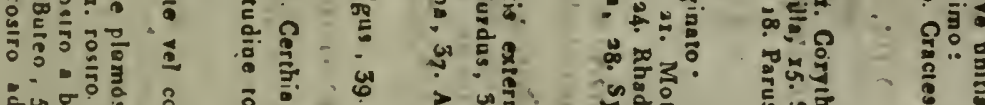

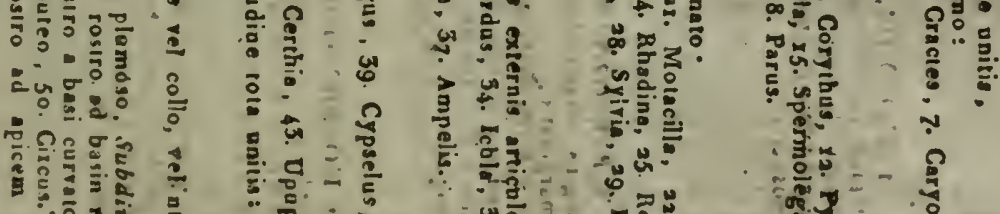

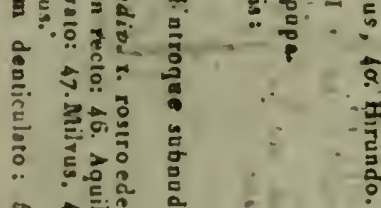

a $\quad-40$

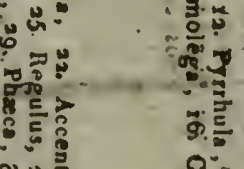

:

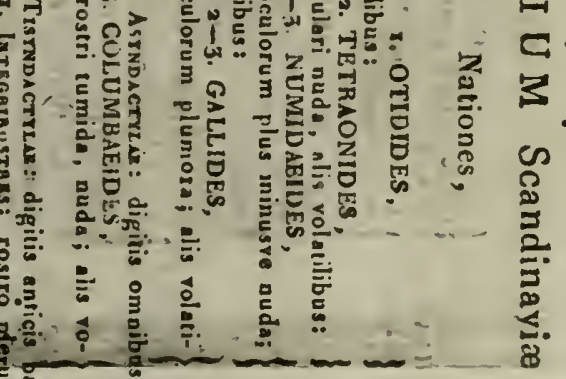

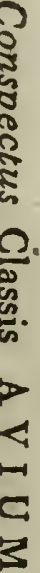

בี่

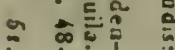

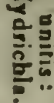

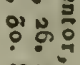

(2)

क्.

要

:

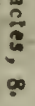

i $\frac{1}{0}$ o

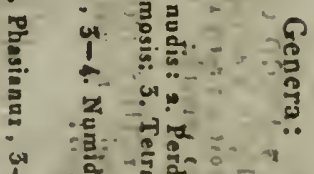

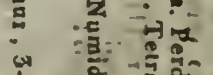

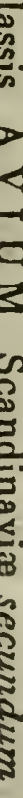


